\title{
Catalytic Asymmetric Conjugate \\ Addition/Hydroalkoxylation Sequence: Expeditious \\ Access to Enantioenriched Eight-Membered Cyclic Ether Derivatives
}

Suo-Suo Qi, Hao Yin, Yi-Feng Wang, ' Chao-Jie Wang, Hong-Te Han, Tong-Tong Man, Dan-Qian $\mathrm{Xu},{ }^{*}$

Catalytic Hydrogenation Research Center, State Key La-boratory Breeding Base of Green ChemistrySynthesis Technology, Key Laboratory of Green Pesticides and Cleaner Production Technology of Zhejiang Province, Zhejiang University of Technology, Hangzhou 310014, P. R. China

E-mail: wangyifeng@zjut.edu.cn; chrc@zjut.edu.cn

\section{List of Contents}

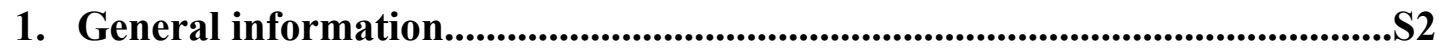

2. General procedure for preparation of ynones..............................................S2

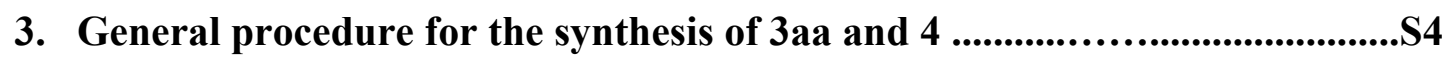

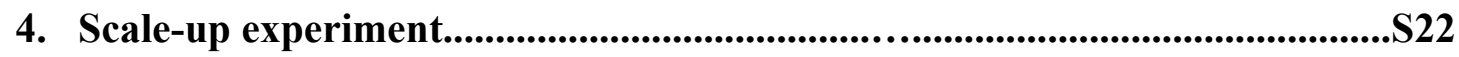

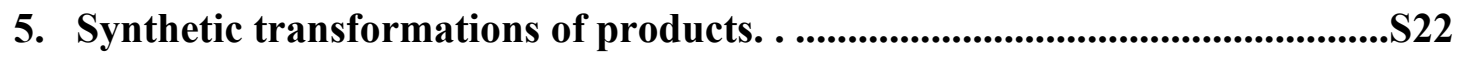

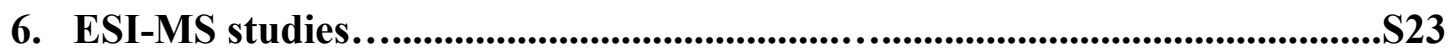

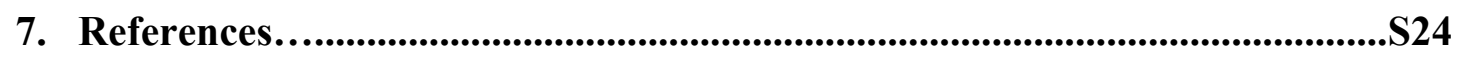

8. HPLC spectra of products...............................................S24

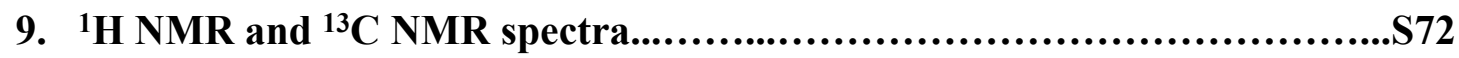

10. Crystal data and structure refinement for enantiopure 4as and 5.........S131

1. General information 
All the starting materials were obtained from commercial sources and used without further purification unless otherwise stated. ${ }^{1} \mathrm{H}$ NMR spectra were recorded on Bruker (400 MHz), Bruker AVANCE III $(500 \mathrm{MHz})$ or Bruker ASCEND $(600 \mathrm{MHz})$ in $\mathrm{CDCl}_{3}$ using residual solvent signals as the internal standard $\left(\mathrm{CDCl}_{3} \delta=7.26 \mathrm{ppm}\right) .{ }^{13} \mathrm{C}$ NMR spectra were recorded at $101 \mathrm{MHz}$ (Bruker), $126 \mathrm{MHz}$ (Bruker AVANCE III) or $151 \mathrm{MHz}$ (Bruker ASCEND) in $\mathrm{CDCl}_{3}$ using solvent signals as the internal standard $\left(\mathrm{CDCl}_{3} \delta=77.16 \mathrm{ppm}\right)$. HRMS data were measured on an Agilent 6120 LC/TOF-MS with ESI source. Melting points (m.p.) were obtained using a Büchi B-545 apparatus and uncorrected. Chiral HPLC analyses were performed using JASCO LC-2000 Plus and Agilent 1260 chromatography (for the compound 4al, 4am, 4an, 4bn, 4gn, 4av and 4gv). Chiralpak IB, IC, ID, AD and IF columns were purchased from Daicel Chemical Industries (Shanghai, China). Optical rotations were measured on a Rudolph Autopol IV polarimeter. Column chromatography and flash chromatography experiments were conducted using silica gel GF254 (200-300 mesh) eluting with ethyl acetate and petroleum ether. TLC experiments were carried out on glass-backed silica plates. The 2-(phenyl(tosyl)methyl)phenols $\mathbf{1}^{[1]}$ and ynones $\mathbf{2}^{[2]}$ were prepared according to the reported literature procedures. All of the 2-(phenyl(tosyl)methyl)phenols are known compounds and the spectral data of unknown ynones are coped as below.

\section{General Procedure for Preparation of ynones.}

Ynones 2d, 2f, 2i, 2q, 2r, 2s, 2v were synthesized according to following steps by using the synthesis of $\mathbf{2 d}$ as example

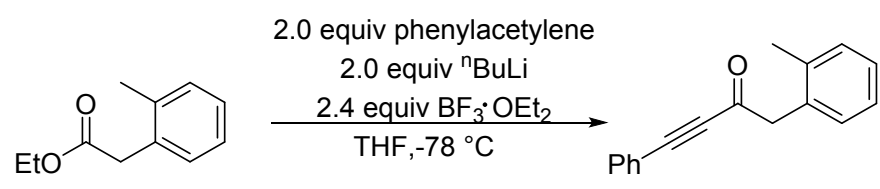

Ethynylbenzene $(0.88 \mathrm{~mL}, 8.0 \mathrm{mmol})$ was dissolved into THF $(30 \mathrm{~mL})$, and the solution was cooled to $-78^{\circ} \mathrm{C}$. To the solution, n-Buli $(5.0 \mathrm{~mL}, 8.0 \mathrm{mmol}, 1.6 \mathrm{M}$ in hexane) was added. After being stirred for $1 \mathrm{~h}$ at $-78{ }^{\circ} \mathrm{C}$, ethyl 2-(o-tolyl)acetate $(0.46 \mathrm{~mL}, 4.0 \mathrm{mmol})$ and $\mathrm{BF}_{3} \cdot \mathrm{OEt}_{2}(1.2 \mathrm{~mL}$, $9.6 \mathrm{mmol}$ ) were added successively. The reaction was quenched by sat. $\mathrm{NH}_{4} \mathrm{Cl}$ aq., and extracted three times with EtOAc. The combined organic layer was dried over $\mathrm{Na}_{2} \mathrm{SO}_{4}$, and the solvent was removed under a reduced pressure. The residue was purified by column chromatography $\left(\mathrm{SiO}_{2}\right.$, eluent: hexane/EtOAc $=95: 5$ ) to afford the desired ynones $2 \mathbf{d}$. The other ynones were synthesized by similar procedure using the corresponding alkynes and esters.<smiles>Cc1ccccc1CC(=O)C#Cc1ccccc1</smiles>

4-phenyl-1-(o-tolyl)but-3-yn-2-one (2d). Purified by silica gel chromatography using PE/EA 10:1, yellow liquid, $67 \%$ yield. ${ }^{1} \mathbf{H}$ NMR $\left(600 \mathrm{MHz}, \mathrm{CDCl}_{3}\right) \delta$ 7.47-7.45 (m, 3H), 7.39-7.36 (m, 2H), 7.31-7.27 (m, 4H), 3.99 (s, 2H), 2.38 (s, 3H). ${ }^{13} \mathbf{C}$ NMR (151 MHz, $\left.\mathrm{CDCl}_{3}\right) \delta$ 185.3, 137.3, 133.2, 132.1, 131.0, 130.9, 130.5, 128.6, 127.8, 126.3, 119.8, 92.7, 87.7, 50.2, 19.8. HRMS (ESI) m/z: [M $+\mathrm{Na}]+$ Calcd for $\mathrm{C}_{17} \mathrm{H}_{14} \mathrm{ONa} 257.0937$; Found 257.0945.<smiles>O=C(C#Cc1ccccc1)Cc1ccccc1F</smiles>

1-(2-fluorophenyl)-4-phenylbut-3-yn-2-one (2f). Purified by silica gel chromatography using PE/EA 10:1, yellow liquid, 54\% yield. ${ }^{1} \mathbf{H}$ NMR $\left(600 \mathrm{MHz}, \mathrm{CDCl}_{3}\right) \delta$ 7.59-7.48 (m, 2H), 7.47-7.44 
(m, 1H), 7.37 (t, $J=7.8 \mathrm{~Hz}, 2 \mathrm{H}), 7.36-7.30(\mathrm{~m}, 2 \mathrm{H}), 7.18(\mathrm{td}, J=7.5,1.1 \mathrm{~Hz}, 1 \mathrm{H}), 7.14(\mathrm{t}, J=9.3$ $\mathrm{Hz}, 1 \mathrm{H}), 4.02(\mathrm{~s}, 2 \mathrm{H}) .{ }^{13} \mathrm{C} \mathrm{NMR}\left(151 \mathrm{MHz}, \mathrm{CDCl}_{3}\right) \delta 183.9,161.3\left(\mathrm{~d},{ }^{1} J_{\mathrm{C}-\mathrm{F}}=246.8 \mathrm{~Hz}\right), 133.2$, $132.0\left(\mathrm{~d},{ }^{4} J_{\mathrm{C}-\mathrm{F}}=3.9 \mathrm{~Hz}\right), 130.9,129.5\left(\mathrm{~d},{ }^{3} J_{\mathrm{C}-\mathrm{F}}=8.1 \mathrm{~Hz}\right), 128.6,124.3\left(\mathrm{~d},{ }^{4} J_{\mathrm{C}-\mathrm{F}}=3.6 \mathrm{~Hz}\right), 120.8(\mathrm{~d}$, $\left.{ }^{2} J_{\mathrm{C}-\mathrm{F}}=16.1 \mathrm{~Hz}\right), 119.7,115.5\left(\mathrm{~d},{ }^{1} J_{\mathrm{C}-\mathrm{F}}=21.7 \mathrm{~Hz}\right), 92.9,87.4,45.3$. HRMS (ESI) m/z: $[\mathrm{M}+\mathrm{Na}]+$ Calcd for $\mathrm{C}_{16} \mathrm{H}_{11} \mathrm{FONa} 261.0686$; Found 261.0694.<smiles>O=C(C#Cc1ccccc1)Cc1ccccc1Cl</smiles>

1-(2-chlorophenyl)-4-phenylbut-3-yn-2-one (2i). Purified by silica gel chromatography using PE/EA 10:1, yellow liquid, $46 \%$ yield. ${ }^{1} \mathbf{H}$ NMR $\left(600 \mathrm{MHz}, \mathrm{CDCl}_{3}\right) \delta$ 7.53-7.51 (m, 2H), 7.48-7.45 (m, 1H), 7.40-7.37 (m, 3H), 7.34-7.32 (m, 2H), $7.22(\mathrm{td}, J=4.8,1.7 \mathrm{~Hz}, 1 \mathrm{H}), 3.93(\mathrm{~s}, 2 \mathrm{H}) .{ }^{13} \mathbf{C}$ NMR $\left(151 \mathrm{MHz}, \mathrm{CDCl}_{3}\right) \delta 184.2,135.1,134.5,133.2,131.0,130.1,129.9,128.7,128.1,127.7$, 119.6, 93.4, 87.6, 51.6. HRMS (ESI) m/z: $[\mathrm{M}+\mathrm{Na}]+$ Calcd for $\mathrm{C}_{16} \mathrm{H}_{11} \mathrm{ClONa} 277.0391$; Found 277.0396 .

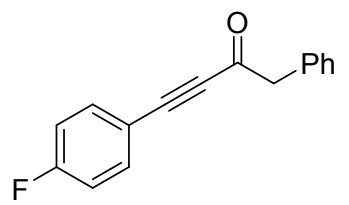

4-(4-fluorophenyl)-1-phenylbut-3-yn-2-one (2q). Purified by silica gel chromatography using PE/EA 10:1, yellow liquid, $61 \%$ yield. ${ }^{1} \mathbf{H}$ NMR $\left(600 \mathrm{MHz}, \mathrm{CDCl}_{3}\right) \delta$ 7.47-7.44 (m, 2H), 7.42-7.39 (m, 2H), 7.36-7.33 (m, 3H), 7.06-7.03 (m, 2H), $3.96(\mathrm{~s}, 2 \mathrm{H}) .{ }^{13} \mathbf{C}$ NMR $\left(151 \mathrm{MHz}, \mathrm{CDCl}_{3}\right) \delta 185.1$, $164.0\left(\mathrm{~d},{ }^{1} J_{\mathrm{C}-\mathrm{F}}=254.0 \mathrm{~Hz}\right), 135.4\left(\mathrm{~d},{ }^{3} J_{\mathrm{C}-\mathrm{F}}=8.9 \mathrm{~Hz}\right), 129.9,128.8,127.5,116.2\left(\mathrm{~d},{ }^{2} J_{\mathrm{C}-\mathrm{F}}=22.3 \mathrm{~Hz}\right)$, 91.9, 87.7, 52.1. HRMS (ESI) m/z: [M + Na]+ Calcd for $\mathrm{C}_{16} \mathrm{H}_{11}$ FONa 261.0686; Found 261.0690.<smiles>O=C(C#Cc1ccccc1Cl)c1ccccc1</smiles>

4-(2-chlorophenyl)-1-phenylbut-3-yn-2-one (2r). Purified by silica gel chromatography using PE/EA 10:1, yellow liquid, 70\% yield. ${ }^{1} \mathbf{H}$ NMR $\left(600 \mathrm{MHz}, \mathrm{CDCl}_{3}\right) \delta$ 7.50-7.49 (m, 1H), 7.44-7.32 (m, 7H), 7.26-7.23 (m, 1H), $4.00(\mathrm{~s}, 2 \mathrm{H}) .{ }^{13} \mathbf{C} \mathbf{N M R}\left(151 \mathrm{MHz}, \mathrm{CDCl}_{3}\right) \delta$ 184.9, 137.4, 134.9, 132.9, 131.9, 130.0, 129.6, 128.8, 127.5, 126.8, 120.1, 91.8, 88.7, 52.3. HRMS (ESI) m/z: $[\mathrm{M}+\mathrm{Na}]+$ Calcd for $\mathrm{C}_{16} \mathrm{H}_{11} \mathrm{ClONa} 277.0391$; Found 277.0397.<smiles>O=C(C#Cc1cccc(Cl)c1)c1ccccc1</smiles>

4-(3-chlorophenyl)-1-phenylbut-3-yn-2-one (2s). Purified by silica gel chromatography using PE/EA 10:1, yellow liquid, $65 \%$ yield. ${ }^{1} \mathbf{H}$ NMR $\left(600 \mathrm{MHz}, \mathrm{CDCl}_{3}\right) \delta$ 7.51-7.49 (m, 2H), 7.48-7.46 (m, 1H), 7.38 (dd, $J=15.7,8.1 \mathrm{~Hz}, 4 \mathrm{H}), 7.29-7.26(\mathrm{~m}, 2 \mathrm{H}), 3.93(\mathrm{~s}, 2 \mathrm{H}) .{ }^{13} \mathbf{C}$ NMR $(151 \mathrm{MHz}$, $\left.\mathrm{CDCl}_{3}\right) \delta 184.6,133.4,133.1,131.7,131.2,131.0,128.9,128.7,119.7,93.3,87.6,51.4$. HRMS (ESI) m/z: [M + Na]+ Calcd for $\mathrm{C}_{16} \mathrm{H}_{11} \mathrm{ClONa} 277.0391$; Found 277.0398.

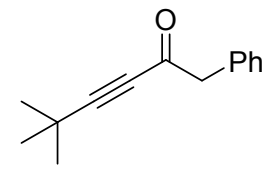


5,5-dimethyl-1-phenylhex-3-yn-2-one (2v). Purified by silica gel chromatography using PE/EA 10:1, colorless liquid, $75 \%$ yield. ${ }^{1} \mathbf{H}$ NMR $\left(600 \mathrm{MHz}, \mathrm{CDCl}_{3}\right) \delta 7.34$ (q, $\left.J=7.0 \mathrm{~Hz}, 2 \mathrm{H}\right), 7.30-7.22$ (m, 3H), $3.80(\mathrm{~s}, 2 \mathrm{H}), 1.21$ (d, $J=1.44 \mathrm{~Hz}, 9 \mathrm{H}) .{ }^{13} \mathbf{C}$ NMR $\left(151 \mathrm{MHz}, \mathrm{CDCl}_{3}\right) \delta 185.6,129.9,128.6$, 127.2, 104.0, 79.2, 52.3, 29.9, 27.7. HRMS (ESI) m/z: [M + Na]+ Calcd for $\mathrm{C}_{14} \mathrm{H}_{16} \mathrm{ONa} 223.1093$; Found 223.1097.

\section{General procedure for the synthesis of 3 aa and 4}

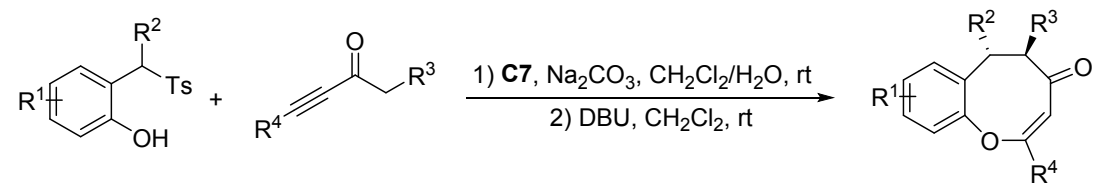

To a test tube were added catalyst $\mathbf{C} 7(0.01 \mathrm{mmol}), \mathrm{Na}_{2} \mathrm{CO}_{3}(0.2 \mathrm{mmol})$, the substituted 2(phenyl(tosyl)methyl)phenol $1(0.1 \mathrm{mmol})$ and ynones $2(0.12 \mathrm{mmol}), 1.5 \mathrm{~mL} \mathrm{DCM}$ and $0.5 \mathrm{~mL}$ $\mathrm{H}_{2} \mathrm{O}$ were then added through syringe. The resulting mixture was stirred at room temperature for 48 h. Then it was extracted with $\mathrm{CH}_{2} \mathrm{Cl}_{2}$, and the organic layer was concentrated under reduced pressure. The resulting residue was directly subjected to flash column chromatography on silica gel (petroleum ether/ethyl acetate $=3: 1$ ) to give the Michael adduct 3. The collected intermediates were dissolved in $\mathrm{CH}_{2} \mathrm{Cl}_{2}(1 \mathrm{~mL})$, then $\mathrm{DBU}(0.02 \mathrm{mmol})$ was added to the solution and the resulting mixture was stirred at $25{ }^{\circ} \mathrm{C}$ until the reaction was detected complete. The solvent was removed under reduced pressure, the resulting residue was purified by column chromatography on silica gel (petroleum ether/ethyl acetate $=4: 1$ ) to give the eight-membered cyclic ethers 4 .<smiles>COc1cccc(C(c2ccccc2)C(C(=O)C#Cc2ccccc2)c2ccccc2)c1</smiles>

(S)-5-(2-hydroxy-3-methoxyphenyl)-1,4,5-triphenylpent-1-yn-3-one (3aa). Purified by silica gel chromatography using PE/EA 3:1, yellow oil, $80 \%$ yield, $34.6 \mathrm{mg}$. 96/90\% ee, 1.8:1 dr. The enantiomeric excess was determined by HPLC on Daicel Chiralpak IF-H with hexane/i-PrOH (90:10) as the eluent, flow $=1.0 \mathrm{~mL} / \mathrm{min}, \mathrm{UV}=254 \mathrm{~nm},[\alpha]_{D}^{25}=-78\left(\mathrm{c}=1.0\right.$ in $\left.\mathrm{CH}_{2} \mathrm{Cl}_{2}\right) .{ }^{1} \mathbf{H}$ NMR $\left(500 \mathrm{MHz}, \mathrm{CDCl}_{3}\right) \delta 7.56(\mathrm{~d}, J=7.5 \mathrm{~Hz}, 2 \mathrm{H}), 7.41-7.36(\mathrm{~m}, 6 \mathrm{H}), 7.24(\mathrm{t}, J=7.3 \mathrm{~Hz}, 5 \mathrm{H})$, $7.10(\mathrm{t}, J=7.5 \mathrm{~Hz}, 2 \mathrm{H}), 6.89-6.83(\mathrm{~m}, 1 \mathrm{H}), 6.73(\mathrm{~d}, J=8.1 \mathrm{~Hz}, 1 \mathrm{H}), 5.92(\mathrm{~d}, J=1 \mathrm{~Hz}, 1 \mathrm{H}), 5.38$ $(\mathrm{d}, J=12.4 \mathrm{~Hz}, 1 \mathrm{H}), 4.98(\mathrm{~d}, J=12.4 \mathrm{~Hz}, 1 \mathrm{H}), 3.81(\mathrm{~d}, J=1.3 \mathrm{~Hz}, 3 \mathrm{H}) .{ }^{13} \mathbf{C}$ NMR $(126 \mathrm{MHz}$, $\left.\mathrm{CDCl}_{3}\right) \delta 186.4,146.6,143.3,141.4,136.0,133.0,130.5,129.3,128.5,128.4,127.9,127.4,126.0$, 120.0, 119.7, 109.0, 87.6, 64.7, 55.8, 46.5. HRMS (ESI) m/z: $[\mathrm{M}+\mathrm{Na}]+$ Calcd for $\mathrm{C}_{30} \mathrm{H}_{24} \mathrm{O}_{3} \mathrm{Na}$ 455.1618; Found 455.1623.

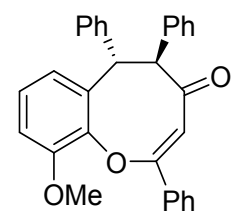

(5S,6S,Z)-10-methoxy-2,5,6-triphenyl-5,6-dihydro-4H-benzo[b]oxocin-4-one (4aa). Purified by silica gel chromatography using PE/EA 4:1, white solid, mp 205-207 ${ }^{\circ} \mathrm{C}, 78 \%$ yield, $33.7 \mathrm{mg}, 96 \%$ $e e,>20: 1 \mathrm{dr}$. The enantiomeric excess was determined by HPLC on Daicel Chiralpak IB-H with hexane/i-PrOH (90:10) as the eluent, flow $=1.0 \mathrm{~mL} / \mathrm{min}, \mathrm{UV}=254 \mathrm{~nm},[\alpha]_{D}^{25}=-274(\mathrm{c}=1.0$ in $\left.\mathrm{CH}_{2} \mathrm{Cl}_{2}\right) .{ }^{1} \mathbf{H}$ NMR $\left(500 \mathrm{MHz}, \mathrm{CDCl}_{3}\right) \delta 8.10-8.09(\mathrm{~m}, 2 \mathrm{H}), 7.58(\mathrm{~d}, J=7.5 \mathrm{~Hz}, 2 \mathrm{H}), 7.55-7.52$ 
(m, 3H), $7.34(\mathrm{~d}, J=8.0 \mathrm{~Hz}, 4 \mathrm{H}), 7.29-7.25(\mathrm{~m}, 3 \mathrm{H}), 7.19(\mathrm{t}, J=7.3 \mathrm{~Hz}, 1 \mathrm{H}), 7.14(\mathrm{t}, J=8.0 \mathrm{~Hz}$, $1 \mathrm{H}), 6.82(\mathrm{~d}, J=8.1 \mathrm{~Hz}, 1 \mathrm{H}), 6.71(\mathrm{~d}, J=7.7 \mathrm{~Hz}, 1 \mathrm{H}), 5.77(\mathrm{~s}, 1 \mathrm{H}), 5.57(\mathrm{~s}, 1 \mathrm{H}), 4.88(\mathrm{~s}, 1 \mathrm{H}), 3.82$ (s, 3H). ${ }^{13} \mathbf{C}$ NMR $\left(126 \mathrm{MHz}, \mathrm{CDCl}_{3}\right) \delta 200.9,161.4,151.6,143.7,139.6,138.5,137.5,134.9,130.4$, 128.7, 128.6, 128.5, 128.4, 128.3, 127.4, 127.3, 127.0, 126.6, 120.1, 110.2, 104.9, 67.2, 55.5, 43.4 . HRMS (ESI) m/z: [M + Na]+ Calcd for $\mathrm{C}_{30} \mathrm{H}_{24} \mathrm{O}_{3} \mathrm{Na}$ 455.1618; Found 455.1623.<smiles>COc1ccc2c(c1)OC(c1ccccc1)CC(=O)C(c1ccccc1)C2c1ccccc1</smiles>

(5S,6S,Z)-9-methoxy-2,5,6-triphenyl-5,6-dihydro-4H-benzo[b]oxocin-4-one (4ba). Purified by silica gel chromatography using PE/EA 4:1, white solid, mp $138-141{ }^{\circ} \mathrm{C}, 70 \%$ yield, $30.2 \mathrm{mg}, 94 \%$ $e e,>20: 1 \mathrm{dr}$. The enantiomeric excess was determined by HPLC on Daicel Chiralpak IB-H with hexane/i-PrOH (90:10) as the eluent, flow $=1.0 \mathrm{~mL} / \mathrm{min}, \mathrm{UV}=254 \mathrm{~nm},[\alpha]_{D}^{25}=-70(\mathrm{c}=1.0 \mathrm{in}$ $\left.\mathrm{CH}_{2} \mathrm{Cl}_{2}\right) .{ }^{1} \mathbf{H}$ NMR $\left(500 \mathrm{MHz}, \mathrm{CDCl}_{3}\right) \delta$ 7.98-7.96 (m, $\left.2 \mathrm{H}\right), 7.55-7.54(\mathrm{~m}, 3 \mathrm{H}), 7.45(\mathrm{~d}, J=7.3 \mathrm{~Hz}$, 2H), 7.28-7.22 (m, 5H), 7.19-7.16 (m, 2H), $7.11(\mathrm{t}, J=7.2 \mathrm{~Hz}, 1 \mathrm{H}), 7.06(\mathrm{~d}, J=8.6 \mathrm{~Hz}, 1 \mathrm{H}), 6.75$ $(\mathrm{d}, J=2.5 \mathrm{~Hz}, 1 \mathrm{H}), 6.71(\mathrm{dd}, J=8.6,2.6 \mathrm{~Hz}, 1 \mathrm{H}), 6.04(\mathrm{~s}, 1 \mathrm{H}), 5.15(\mathrm{~s}, 1 \mathrm{H}), 4.97(\mathrm{~s}, 1 \mathrm{H}), 3.73(\mathrm{~s}$, 3H). ${ }^{13} \mathrm{C}$ NMR $\left(126 \mathrm{MHz}, \mathrm{CDCl}_{3}\right) \delta 202.0,159.3,137.9,134.4,131.5,130.8,129.2,128.9,128.4$, $128.3,128.1,127.3,126.7,126.6,113.0,107.1,100.0,64.5,55.5,49.2$. HRMS (ESI) m/z: [M + $\mathrm{Na}]+$ Calcd for $\mathrm{C}_{30} \mathrm{H}_{24} \mathrm{O}_{3} \mathrm{Na}$ 455.1618; Found 455.1624.<smiles>CCOc1cccc2c1OC(c1ccccc1)CC(=O)C(c1ccccc1)c1ccccc1-2</smiles>

(5S,6S,Z)-10-ethoxy-2,5,6-triphenyl-5,6-dihydro-4H-benzo[b]oxocin-4-one (4ca). Purified by silica gel chromatography using PE/EA 4:1, white solid, mp $179-182{ }^{\circ} \mathrm{C}, 80 \%$ yield, $35.7 \mathrm{mg}, 95 \%$ $e e,>20: 1 \mathrm{dr}$. The enantiomeric excess was determined by HPLC on Daicel Chiralpak IC-H with hexane/i-PrOH (90:10) as the eluent, flow $=1.0 \mathrm{~mL} / \mathrm{min}, \mathrm{UV}=254 \mathrm{~nm},[\alpha]_{D}^{25}=-24^{3}(\mathrm{c}=1.0$ in $\left.\mathrm{CH}_{2} \mathrm{Cl}_{2}\right) .{ }^{1} \mathbf{H}$ NMR $\left(500 \mathrm{MHz}, \mathrm{CDCl}_{3}\right) \delta 8.09(\mathrm{~d}, J=6.9 \mathrm{~Hz}, 2 \mathrm{H}), 7.54-7.49(\mathrm{~m}, 5 \mathrm{H}), 7.32-7.28$ (m, 4H), 7.25-7.23 (m, 3H), $7.16(\mathrm{t}, J=7.2 \mathrm{~Hz}, 1 \mathrm{H}), 7.08(\mathrm{t}, J=8.0 \mathrm{~Hz}, 1 \mathrm{H}), 6.78(\mathrm{~d}, J=8.1 \mathrm{~Hz}$, 1H), $6.66(\mathrm{~d}, J=7.3 \mathrm{~Hz}, 1 \mathrm{H}), 5.75$ (s, 1H), $5.46(\mathrm{~s}, 1 \mathrm{H}), 4.86$ (s, 2H), 5.9 (d, $J=5.9 \mathrm{~Hz}, 2 \mathrm{H}), 1.32$ (s, 3H). ${ }^{13} \mathbf{C}$ NMR $\left(126 \mathrm{MHz}, \mathrm{CDCl}_{3}\right) \delta 201.1,151.2,144.1,140.0,138.5,137.7,135.2,130.5,128.9$, $128.8,128.6,128.5,128.4,127.5,127.1,126.8,120.2,111.0,105.2,67.3,64.2,53.5,14.6$. HRMS (ESI) $\mathrm{m} / \mathrm{z}:[\mathrm{M}+\mathrm{Na}]+$ Calcd for $\mathrm{C}_{31} \mathrm{H}_{26} \mathrm{O}_{3} \mathrm{Na}$ 469.1774; Found 469.1781 .<smiles>Cc1ccc2c(c1)[C@@H](c1ccccc1)C(c1ccccc1)C=C(c1ccccc1)O2</smiles>

(5S,6S,Z)-8-methyl-2,5,6-triphenyl-5,6-dihydro-4H-benzo[b]oxocin-4-one (4da). Purified by silica gel chromatography using PE/EA 4:1, white solid, mp 210-212 ${ }^{\circ} \mathrm{C}, 54 \%$ yield, $22.4 \mathrm{mg}, 87 \%$ $e e,>20: 1 \mathrm{dr}$. The enantiomeric excess was determined by HPLC on Daicel Chiralpak IB-H with hexane/i-PrOH (90:10) as the eluent, flow $=1.0 \mathrm{~mL} / \mathrm{min}, \mathrm{UV}=254 \mathrm{~nm},[\alpha]_{D}^{25}=-83(\mathrm{c}=1.0 \mathrm{in}$ $\left.\mathrm{CH}_{2} \mathrm{Cl}_{2}\right) .{ }^{1} \mathbf{H}$ NMR $\left(500 \mathrm{MHz}, \mathrm{CDCl}_{3}\right) \delta$ 8.00-7.97 (m, 2H), 7.55-7.53 (m, 3H), 7.45-7.44 (d, $J=7.4$ 
$\mathrm{Hz}, 2 \mathrm{H}), 7.27-7.24(\mathrm{~m}, 4 \mathrm{H}), 7.21-7.17(\mathrm{~m}, 3 \mathrm{H}), 7.11(\mathrm{t}, J=8.2 \mathrm{~Hz}, 2 \mathrm{H}), 6.96-6.95(\mathrm{~m}, 2 \mathrm{H}), 6.06(\mathrm{~s}$, 1H), 5.22 (s, 1H), 4.91 (s, 1H), 2.23 (s, 3H). ${ }^{13} \mathbf{C}$ NMR (126 MHz, CDCl3) $\delta$ 202.1, 154.4, 141.8, 138.0, 136.4, 135.3, 134.4, 131.3, 130.8, 129.3, 129.0, 128.9, 128.4, 128.4, 128.2, 127.2, 126.7, 126.6, 121.4, 64.3, 53.4, 20.8. HRMS (ESI) m/z: [M+ Na]+ Calcd for $\mathrm{C}_{30} \mathrm{H}_{24} \mathrm{O}_{2} \mathrm{Na}$ 439.1669; Found 439.1673 .

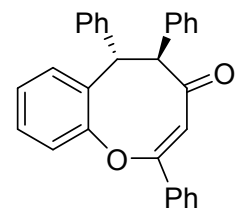

(5S,6S,Z)-2,5,6-triphenyl-5,6-dihydro-4H-benzo[b]oxocin-4-one (4ea). Purified by silica gel chromatography using PE/EA 4:1, white solid, mp 102-105 ${ }^{\circ} \mathrm{C}, 50 \%$ yield, $20.1 \mathrm{mg}, 86 \%$ ee, $>20: 1$ dr. The enantiomeric excess was determined by HPLC on Daicel Chiralpak IC-H with hexane/i$\operatorname{PrOH}(90: 10)$ as the eluent, flow $=1.0 \mathrm{~mL} / \mathrm{min}, \mathrm{UV}=254 \mathrm{~nm},[\alpha]_{D}^{25}=-56\left(\mathrm{c}=1.0\right.$ in $\left.\mathrm{CH}_{2} \mathrm{Cl}_{2}\right)$. ${ }^{1} \mathbf{H}$ NMR $\left(500 \mathrm{MHz}, \mathrm{CDCl}_{3}\right) \delta$ 7.99-7.98 (m, 2H), 7.56-7.54 (m, 3H), $7.46(\mathrm{~d}, J=7.2 \mathrm{~Hz}, 2 \mathrm{H}), 7.28$ $7.23(\mathrm{~m}, 5 \mathrm{H}), 7.22-7.19(\mathrm{~m}, 2 \mathrm{H}), 7.18-7.16(\mathrm{~m}, 3 \mathrm{H}), 7.15-7.11(\mathrm{~m}, 2 \mathrm{H}), 6.06(\mathrm{~s}, 1 \mathrm{H}), 5.21(\mathrm{~s}, 1 \mathrm{H})$, 5.00 (s, 1H). ${ }^{13} \mathrm{C}$ NMR $\left(126 \mathrm{MHz}, \mathrm{CDCl}_{3}\right) \delta 201.9,164.6,156.2,141.5,137.8,135.9,134.4,131.0$, 130.9, 129.2, 128.9, 128.4, 128.4, 128.3, 128.3, 127.3, 126.8, 126.7, 126.6, 121.7, 110.6, 64.4, 50.1 . HRMS (ESI) m/z: [M + Na]+ Calcd for $\mathrm{C}_{29} \mathrm{H}_{22} \mathrm{O}_{2} \mathrm{Na}$ 425.1512; Found 425.1518.<smiles></smiles>

(5S,6S,Z)-9-fluoro-2,5,6-triphenyl-5,6-dihydro-4H-benzo[b]oxocin-4-one (4fa). Purified by silica gel chromatography using PE/EA 4:1, white solid, mp 95-98 ${ }^{\circ} \mathrm{C}, 61 \%$ yield, $25.6 \mathrm{mg}, 89 \%$ ee, $>20: 1 \mathrm{dr}$. The enantiomeric excess was determined by HPLC on Daicel Chiralpak IB-H with hexane/i-PrOH (90:10) as the eluent, flow $=1.0 \mathrm{~mL} / \mathrm{min}, \mathrm{UV}=254 \mathrm{~nm},[\alpha]_{D}^{25}=-85(\mathrm{c}=1.0 \mathrm{in}$ $\mathrm{CH}_{2} \mathrm{Cl}_{2}$ ). ${ }^{1} \mathbf{H}$ NMR $\left(600 \mathrm{MHz}, \mathrm{CDCl}_{3}\right) \delta$ 7.99-7.98 (m, 2H), 7.58-7.57 (m, 3H), 7.49 (d, J=7.4 Hz, 2H), 7.30-7.25 (m, 5H), 7.23-7.20 (m, 2H), 7.18-7.14 (m, 2H), 7.00-6.97 (m, 1H), 6.91-6.88 (m, $1 \mathrm{H}), 6.15(\mathrm{~s}, 1 \mathrm{H}), 5.27(\mathrm{~s}, 1 \mathrm{H}), 4.98(\mathrm{~s}, 1 \mathrm{H}) .{ }^{13} \mathbf{C}$ NMR $\left(126 \mathrm{MHz}, \mathrm{CDCl}_{3}\right) \delta 201.6,164.1,161.4(\mathrm{~d}$, $\left.{ }^{1} J_{\mathrm{C}-\mathrm{F}}=247.6 \mathrm{~Hz}\right), 156.5,141.3,137.6,133.8,132.3\left(\mathrm{~d},{ }^{3} J_{\mathrm{C}-\mathrm{F}}=8.0 \mathrm{~Hz}\right), 131.9,131.0,129.2,128.9$, $128.4,128.2,128.2,127.3,126.7,126.5,114.2\left(\mathrm{~d},{ }^{2} J_{\mathrm{C}-\mathrm{F}}=21.0 \mathrm{~Hz}\right), 111.2,109.2\left(\mathrm{~d},{ }^{2} J_{\mathrm{C}-\mathrm{F}}=22.9\right.$ $\mathrm{Hz}$ ), 64.0, 50.1. HRMS (ESI) m/z: [M + Na]+ Calcd for $\mathrm{C}_{29} \mathrm{H}_{21} \mathrm{O}_{2} \mathrm{FNa}$ 443.1418; Found 443.1427.<smiles>O=C1C=C(c2ccccc2)Oc2c(F)cccc2C1c1ccccc1</smiles>

(5S,6S,Z)-10-fluoro-2,5,6-triphenyl-5,6-dihydro-4H-benzo[b]oxocin-4-one (4ga). Purified by silica gel chromatography using PE/EA 4:1, white solid, mp $92-94{ }^{\circ} \mathrm{C}, 75 \%$ yield, $31.5 \mathrm{mg}, 92 \%$ ee, $>20: 1 \mathrm{dr}$. The enantiomeric excess was determined by HPLC on Daicel Chiralpak IB-H with hexane/i-PrOH (90:10) as the eluent, flow $=1.0 \mathrm{~mL} / \mathrm{min}, \mathrm{UV}=254 \mathrm{~nm},[\alpha]_{D}^{25}=-243$ (c $=1.0$ in $\left.\mathrm{CH}_{2} \mathrm{Cl}_{2}\right) .{ }^{1} \mathbf{H}$ NMR $(500 \mathrm{MHz}, \mathrm{CDCl} 3) \delta 8.0-7.98(\mathrm{~m}, 2 \mathrm{H}), 7.54-7.49(\mathrm{~m}, 5 \mathrm{H}), 7.31-7.29(\mathrm{~m}, 4 \mathrm{H})$, $7.24(\mathrm{t}, J=7.4 \mathrm{~Hz}, 3 \mathrm{H}), 7.17(\mathrm{t}, J=7.2 \mathrm{~Hz}, 1 \mathrm{H}), 7.11(\mathrm{td}, J=8.0,5.1 \mathrm{~Hz}, 1 \mathrm{H}), 7.04-7.00(\mathrm{~m}, 1 \mathrm{H})$, $6.90(\mathrm{~d}, J=7.8 \mathrm{~Hz}, 1 \mathrm{H}), 5.92(\mathrm{~s}, 1 \mathrm{H}), 5.28(\mathrm{~s}, 1 \mathrm{H}), 4.98(\mathrm{~s}, 1 \mathrm{H}) .{ }^{13} \mathbf{C} \mathbf{N M R}\left(126 \mathrm{MHz}, \mathrm{CDCl}_{3}\right) \delta$ $200.9,162.3,154.7\left(\mathrm{~d},{ }^{1} J_{\mathrm{C}-\mathrm{F}}=248.6 \mathrm{~Hz}\right), 143.1,139.9,139.5,137.2,133.9,130.8,128.9,128.6$, 
$128.6,128.6,128.5,127.5,127.2\left(\mathrm{~d},{ }^{3} J_{\mathrm{C}-\mathrm{F}}=7.7 \mathrm{~Hz}\right), 127.0,126.9,124.9,115.1\left(\mathrm{~d},{ }^{2} J_{\mathrm{C}-\mathrm{F}}=18.8 \mathrm{~Hz}\right)$, 107.7, 65.9, 47.0. HRMS (ESI) m/z: [M + Na]+ Calcd for $\mathrm{C}_{29} \mathrm{H}_{21} \mathrm{O}_{2} \mathrm{FNa} 443.1418$; Found 443.1425.<smiles></smiles>

(5S,6S,Z)-8-chloro-2,5,6-triphenyl-5,6-dihydro-4H-benzo[b]oxocin-4-one (4ha). Purified by silica gel chromatography using PE/EA 4:1, white solid, mp 107-109 ${ }^{\circ} \mathrm{C}, 48 \%$ yield, $20.9 \mathrm{mg}, 90 \%$ $e e,>20: 1 \mathrm{dr}$. The enantiomeric excess was determined by HPLC on Daicel Chiralpak IC-H with hexane/i-PrOH (90:10) as the eluent, flow $=1.0 \mathrm{~mL} / \mathrm{min}, \mathrm{UV}=254 \mathrm{~nm},[\alpha]_{D}^{25}=-116(\mathrm{c}=1.0$ in $\mathrm{CH}_{2} \mathrm{Cl}_{2}$ ). ${ }^{1} \mathrm{H}$ NMR (500 MHz, Chloroform- $\left.d\right)^{1} \mathbf{H}$ NMR $\left(500 \mathrm{MHz}, \mathrm{CDCl}_{3}\right) \delta$ 7.97-7.95 (m, 2H), 7.56-7.54 (m, 3H), 7.44-7.42 (m, 2H), 7.28-7.17 (m, 8H), 7.15-7.14 (m, 3H), $6.10(\mathrm{~s}, 1 \mathrm{H}), 5.21(\mathrm{~s}$, 1H), $4.90(\mathrm{~s}, 1 \mathrm{H}) .{ }^{13} \mathbf{C}$ NMR $\left(126 \mathrm{MHz}, \mathrm{CDCl}_{3}\right) \delta 200.7,151.8,138.0,137.3,134.9,132.5,130.5$, 130.1, 128.7, 128.6, 128.4, 127.6, 127.4, 127.2, 120.1, 110.6, 104.9, 67.1, 55.6, 41.0. HRMS (ESI) $\mathrm{m} / \mathrm{z}:[\mathrm{M}+\mathrm{Na}]+$ Calcd for $\mathrm{C}_{29} \mathrm{H}_{21} \mathrm{O}_{2} \mathrm{ClNa} 459.1122$; Found 459.1130 .<smiles>O=C1C=C(c2ccccc2)C(c2ccccc2)c2cccc(C(F)(F)F)c2OC1c1ccccc1</smiles>

(5S,6S,Z)-2,5,6-triphenyl-10-(trifluoromethyl)-5,6-dihydro-4H-benzo[b]oxocin-4-one (4ia). Purified by silica gel chromatography using PE/EA 4:1, white solid, mp $87-89^{\circ} \mathrm{C}, 76 \%$ yield, 33.1 $\mathrm{mg}, 85 \% e e,>20: 1 \mathrm{dr}$. The enantiomeric excess was determined by HPLC on Daicel Chiralpak ID-H with hexane/i-PrOH (90:10) as the eluent, flow $=1.0 \mathrm{~mL} / \mathrm{min}, \mathrm{UV}=254 \mathrm{~nm},[\alpha]_{D}^{25}=-153(\mathrm{c}=$ 1.0 in $\left.\mathrm{CH}_{2} \mathrm{Cl}_{2}\right) .{ }^{1} \mathbf{H}$ NMR $\left(500 \mathrm{MHz}, \mathrm{CDCl}_{3}\right) \delta 7.90-7.88(\mathrm{~m}, 2 \mathrm{H}), 7.58(\mathrm{dd}, J=7.5,1.4 \mathrm{~Hz}, 1 \mathrm{H})$, 7.53-7.47 (m, 5H), 7.31-7.23 (m, 9H), 7.22-7.19 (m, 1H), $5.73(\mathrm{~d}, J=12.7 \mathrm{~Hz}, 1 \mathrm{H}), 5.65(\mathrm{~s}, 1 \mathrm{H})$, $4.54(\mathrm{~d}, J=12.7 \mathrm{~Hz}, 1 \mathrm{H}) .{ }^{13} \mathbf{C}$ NMR $\left(126 \mathrm{MHz}, \mathrm{CDCl}_{3}\right) \delta 200.2,157.4,149.8,139.8,138.1,136.6$, $133.8,132.8,130.5,129.2,128.9,128.8,128.7,128.3,127.8,127.2,127.0,127.0,125.7\left(\mathrm{q},{ }^{3} J_{\mathrm{C}-\mathrm{F}}=\right.$ $5.1 \mathrm{~Hz}), 123.0\left(\mathrm{q},{ }^{1} J_{\mathrm{C}-\mathrm{F}}=271.4 \mathrm{~Hz}\right) 122.8\left(\mathrm{q},{ }^{2} J_{\mathrm{C}-\mathrm{F}}=31.3 \mathrm{~Hz}\right), 101.5,67.8,41.1$. HRMS (ESI) m/z: $[\mathrm{M}+\mathrm{Na}]+$ Calcd for $\mathrm{C}_{30} \mathrm{H}_{21} \mathrm{~F}_{3} \mathrm{O}_{2} \mathrm{Na}$ 493.1386; Found 493.1394.<smiles>O=C1/C=C(\c2ccccc2)C(c2ccccc2)c2cc(Br)cc(Br)c2OC1c1ccccc1</smiles>

(5S,6S,Z)-8,10-dibromo-2,5,6-triphenyl-5,6-dihydro-4H-benzo[b]oxocin-4-one (4ja). Purified by silica gel chromatography using PE/EA 4:1, white solid, mp $220-222{ }^{\circ} \mathrm{C}, 73 \%$ yield, $40.8 \mathrm{mg}, 85 \%$ $e e,>20: 1 \mathrm{dr}$. The enantiomeric excess was determined by HPLC on Daicel Chiralpak ID-H with hexane/i-PrOH (90:10) as the eluent, flow $=1.0 \mathrm{~mL} / \mathrm{min}, \mathrm{UV}=254 \mathrm{~nm},[\alpha]_{D}^{25}=-344(\mathrm{c}=1.0$ in $\left.\mathrm{CH}_{2} \mathrm{Cl}_{2}\right) .{ }^{1} \mathbf{H}$ NMR $(500 \mathrm{MHz}, \mathrm{CDCl} 3) \delta 8.04-8.02(\mathrm{~m}, 2 \mathrm{H}), 7.64(\mathrm{~d}, J=2.3 \mathrm{~Hz}, 1 \mathrm{H}), 7.53-7.49$ $(\mathrm{m}, 3 \mathrm{H}), 7.46(\mathrm{~d}, J=7.3 \mathrm{~Hz}, 2 \mathrm{H}), 7.30-7.27(\mathrm{~m}, 6 \mathrm{H}), 7.24-7.21(\mathrm{~m}, 2 \mathrm{H}), 7.08(\mathrm{~d}, J=2.3 \mathrm{~Hz}, 1 \mathrm{H})$, $5.69(\mathrm{~s}, 1 \mathrm{H}), 5.64(\mathrm{~d}, J=12.7 \mathrm{~Hz}, 1 \mathrm{H}), 4.57(\mathrm{~d}, J=12.7 \mathrm{~Hz}, 1 \mathrm{H}) .{ }^{13} \mathbf{C}$ NMR $\left(126 \mathrm{MHz}, \mathrm{CDCl}_{3}\right) \delta$ $199.8,157.5,149.3,141.5,137.5,136.4,134.5,133.7,130.8,130.6,128.9,128.9,128.6,128.3$, $127.8,127.4,127.2,120.6,117.4,103.0,68.2,43.0$. HRMS (ESI) $\mathrm{m} / \mathrm{z}:[\mathrm{M}+\mathrm{Na}]+$ Calcd for $\mathrm{C}_{29} \mathrm{H}_{20} \mathrm{Br}_{2} \mathrm{O}_{2} \mathrm{Na} 580.9722$; Found 580.9730 . 


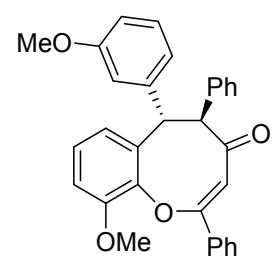

(5S,6S,Z)-10-methoxy-6-(3-methoxyphenyl)-2,5-diphenyl-5,6-dihydro-4H-benzo[b]oxocin-4one (4ka). Purified by silica gel chromatography using PE/EA 4:1, colorless oil, 77\% yield, 35.5 $\mathrm{mg}, 97 \% e e,>20: 1 \mathrm{dr}$. The enantiomeric excess was determined by HPLC on Daicel Chiralpak IB-H with hexane/i-PrOH (90:10) as the eluent, flow $=1.0 \mathrm{~mL} / \mathrm{min}, \mathrm{UV}=254 \mathrm{~nm},[\alpha]_{D}^{25}=-427(\mathrm{c}=$ 1.0 in $\left.\mathrm{CH}_{2} \mathrm{Cl}_{2}\right) .{ }^{1} \mathbf{H}$ NMR $\left(500 \mathrm{MHz}, \mathrm{CDCl}_{3}\right) \delta$ 8.04-8.02 (m, 2H), 7.53-7.49 (m, 5H), 7.31-7.28 (m, 2H), 7.24-7.21 (m, 1H), $7.15(\mathrm{t}, J=8.0 \mathrm{~Hz}, 1 \mathrm{H}), 7.11(\mathrm{t}, J=8.1 \mathrm{~Hz}, 1 \mathrm{H}), 6.89(\mathrm{~d}, J=7.7 \mathrm{~Hz}, 1 \mathrm{H})$, 6.84-6.82 (m, 1H), $6.80(\mathrm{dd}, J=8.2,1.1 \mathrm{~Hz}, 1 \mathrm{H}), 6.71-6.68(\mathrm{~m}, 2 \mathrm{H}), 5.69(\mathrm{~s}, 1 \mathrm{H}), 5.46(\mathrm{~s}, 1 \mathrm{H}), 4.77$ (s, 1H), $3.82(\mathrm{~s}, 3 \mathrm{H}), 3.70(\mathrm{~s}, 3 \mathrm{H}) .{ }^{13} \mathbf{C}$ NMR $\left(126 \mathrm{MHz}, \mathrm{CDCl}_{3}\right) \delta 200.9,159.6,151.6,141.3,138.4$, 137.6, 135.1, 130.5, 129.4, 128.7, 128.6, 128.4, 127.5, 127.4, 127.1, 121.1, 120.2, 114.7, 112.2, 110.4, 67.2, 55.7, 55.1, 48.1. HRMS (ESI) $\mathrm{m} / \mathrm{z}$ : $[\mathrm{M}+\mathrm{Na}]+\mathrm{Calcd}$ for $\mathrm{C}_{31} \mathrm{H}_{26} \mathrm{O}_{4} \mathrm{Na}$ 485.1723; Found 485.1730 .

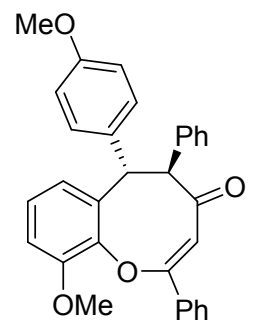

(5S,6S,Z)-10-methoxy-6-(4-methoxyphenyl)-2,5-diphenyl-5,6-dihydro-4H-benzo[b]oxocin-4one (4la). Purified by silica gel chromatography using PE/EA 4:1, white solid, mp $226-228^{\circ} \mathrm{C}, 82 \%$ yield, $37.9 \mathrm{mg}, 97 \% e e,>20: 1 \mathrm{dr}$. The enantiomeric excess was determined by HPLC on Daicel Chiralpak IB-H with hexane/i-PrOH (90:10) as the eluent, flow $=1.0 \mathrm{~mL} / \mathrm{min}, \mathrm{UV}=254 \mathrm{~nm},[\alpha]_{D}^{25}$ $=-340\left(\mathrm{c}=1.0\right.$ in $\left.\mathrm{CH}_{2} \mathrm{Cl}_{2}\right) .{ }^{1} \mathbf{H}$ NMR $\left(500 \mathrm{MHz}, \mathrm{CDCl}_{3}\right) \delta 8.04-8.03(\mathrm{~m}, 2 \mathrm{H}), 7.52-7.50(\mathrm{~m}$, $5 \mathrm{H}), 7.31-7.28(\mathrm{~m}, 2 \mathrm{H}), 7.24-7.20(\mathrm{~m}, 3 \mathrm{H}), 7.11(\mathrm{t}, J=8.0 \mathrm{~Hz}, 1 \mathrm{H}), 6.80-6.77(\mathrm{~m}, 3 \mathrm{H}), 6.66(\mathrm{~d}, J=$ $7.8 \mathrm{~Hz}, 1 \mathrm{H}), 5.69(\mathrm{~s}, 1 \mathrm{H}), 5.45(\mathrm{~s}, 1 \mathrm{H}), 4.74(\mathrm{~s}, 1 \mathrm{H}), 3.82(\mathrm{~s}, 3 \mathrm{H}), 3.73(\mathrm{~s}, 3 \mathrm{H}) .{ }^{13} \mathbf{C ~ N M R}(126 \mathrm{MHz}$, $\left.\mathrm{CDCl}_{3}\right) \delta 201.1,158.2,151.6,138.8,137.7,135.1,130.4,129.7,128.7,128.6,128.4,127.4,127.1$, 120.2, 113.9, 110.2, 67.6, 55.6, 55.1, 43.5. HRMS (ESI) m/z: $[\mathrm{M}+\mathrm{Na}]+$ Calcd for $\mathrm{C}_{31} \mathrm{H}_{26} \mathrm{O}_{4} \mathrm{Na}$ 485.1723; Found 485.1728.

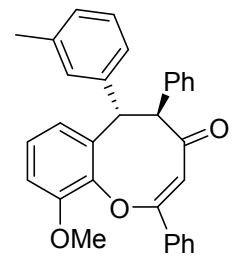

(5S,6S,Z)-10-methoxy-2,5-diphenyl-6-(m-tolyl)-5,6-dihydro-4H-benzo[b]oxocin-4-one (4ma). Purified by silica gel chromatography using PE/EA 4:1, white solid, mp $119-121^{\circ} \mathrm{C}, 70 \%$ yield, $31.2 \mathrm{mg}, 97 \% e e,>20: 1 \mathrm{dr}$. The enantiomeric excess was determined by HPLC on Daicel Chiralpak IB-H with hexane/i-PrOH (90:10) as the eluent, flow $=1.0 \mathrm{~mL} / \mathrm{min}, \mathrm{UV}=254 \mathrm{~nm},[\alpha]_{D}^{25}=-332$ (c = 1.0 in $\left.\mathrm{CH}_{2} \mathrm{Cl}_{2}\right) .{ }^{1} \mathbf{H}$ NMR $\left(500 \mathrm{MHz}, \mathrm{CDCl}_{3}\right)^{\mathbf{1}} \mathbf{H} \mathbf{~ N M R}\left(500 \mathrm{MHz}, \mathrm{CDCl}_{3}\right) \delta 8.04-8.02(\mathrm{~m}, 2 \mathrm{H})$, 7.52-7.47 (m, 5H), 7.30-7.27 (m, 2H), 7.23-7.20 (m, 1H), 7.12-7.09 (m, 4H), $6.96(\mathrm{~d}, J=6.2 \mathrm{~Hz}$, $1 \mathrm{H}), 6.79(\mathrm{dd}, J=8.2,1.4 \mathrm{~Hz}, 1 \mathrm{H}), 6.66(\mathrm{~d}, J=7.7 \mathrm{~Hz}, 1 \mathrm{H}), 5.67(\mathrm{~s}, 1 \mathrm{H}), 5.47(\mathrm{~s}, 1 \mathrm{H}), 4.76(\mathrm{~s}, 1 \mathrm{H})$, 
$3.82(\mathrm{~s}, 3 \mathrm{H}), 2.25(\mathrm{~s}, 3 \mathrm{H}) .{ }^{13} \mathbf{C}$ NMR $\left(126 \mathrm{MHz}, \mathrm{CDCl}_{3}\right) \delta 200.9,161.4,151.5,143.8,139.4,138.7$, 137.9, 137.6, 135.0, 130.4, 129.8, 128.6, 128.5, 128.3, 128.2, 127.5, 127.4, 127.0, 125.5, 120.1, 110.2, 105.1, 67.2, 55.5, 44.0, 21.3. HRMS (ESI) m/z: [M + Na]+ Calcd for $\mathrm{C}_{31} \mathrm{H}_{26} \mathrm{O}_{3} \mathrm{Na}$ 469.1774; Found 469.1781 .

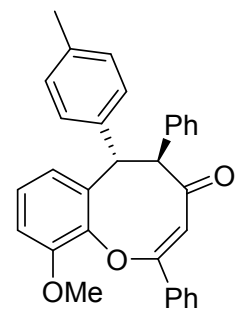

(5S,6S,Z)-10-methoxy-2,5-diphenyl-6-(p-tolyl)-5,6-dihydro-4H-benzo[b]oxocin-4-one (4na). Purified by silica gel chromatography using PE/EA 4:1, white solid, mp $134-137^{\circ} \mathrm{C}, 80 \%$ yield, $35.6 \mathrm{mg}, 97 \% e e,>20: 1 \mathrm{dr}$. The enantiomeric excess was determined by HPLC on Daicel Chiralpak IB-H with hexane/i-PrOH (90:10) as the eluent, flow $=1.0 \mathrm{~mL} / \mathrm{min}, \mathrm{UV}=254 \mathrm{~nm},[\alpha]_{D}^{25}=-343$ (c = 1.0 in $\left.\mathrm{CH}_{2} \mathrm{Cl}_{2}\right) .{ }^{1} \mathbf{H}$ NMR $\left(500 \mathrm{MHz}, \mathrm{CDCl}_{3}\right) \delta 8.08-8.07(\mathrm{~m}, 1 \mathrm{H}), 7.56(\mathrm{~d}, J=7.4 \mathrm{~Hz}, 2 \mathrm{H})$, $7.53(\mathrm{~d}, J=5.1 \mathrm{~Hz}, 3 \mathrm{H}), 7.32(\mathrm{t}, J=7.3 \mathrm{~Hz}, 2 \mathrm{H}), 7.26-7.21(\mathrm{~m}, 3 \mathrm{H}), 7.13(\mathrm{t}, J=8.0 \mathrm{~Hz}, 1 \mathrm{H}), 7.07$ $(\mathrm{d}, J=7.5 \mathrm{~Hz}, 2 \mathrm{H}), 6.81(\mathrm{~d}, J=8.1 \mathrm{~Hz}, 1 \mathrm{H}), 6.70(\mathrm{~d}, J=7.6 \mathrm{~Hz}, 1 \mathrm{H}), 5.72(\mathrm{~s}, 1 \mathrm{H}), 5.54(\mathrm{~s}, 1 \mathrm{H})$, $4.81(\mathrm{~s}, 1 \mathrm{H}), 3.83$ (s, 3H), 2.29 (s, 3H). ${ }^{13} \mathbf{C}$ NMR $\left(126 \mathrm{MHz}, \mathrm{CDCl}_{3}\right) \delta 200.8,161.1,151.5,143.8$, 138.8, 137.6, 136.6, 136.1, 135.0, 130.3, 129.1, 128.6, 128.5, 128.5, 128.3, 127.3, 127.0, 120.0, 110.2, 104.8, 67.4, 55.5, 43.3, 20.9. HRMS (ESI) m/z: [M + Na]+ Calcd for $\mathrm{C}_{31} \mathrm{H}_{26} \mathrm{O}_{3} \mathrm{Na}$ 469.1774; Found 469.1787.

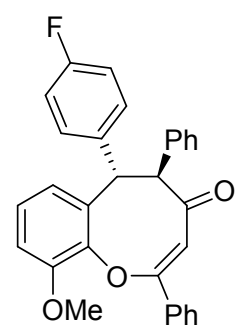

(5S,6S,Z)-6-(4-fluorophenyl)-10-methoxy-2,5-diphenyl-5,6-dihydro-4H-benzo[b]oxocin-4-one (4oa). Purified by silica gel chromatography using PE/EA 4:1, white solid, mp $220-222^{\circ} \mathrm{C}, 81 \%$ yield, $36.4 \mathrm{mg}, 96 \%$ ee, $>20: 1 \mathrm{dr}$. The enantiomeric excess was determined by HPLC on Daicel Chiralpak IB-H with hexane/i-PrOH (90:10) as the eluent, flow $=1.0 \mathrm{~mL} / \mathrm{min}, \mathrm{UV}=254 \mathrm{~nm},[\alpha]_{D}^{25}$ $=-312\left(\mathrm{c}=1.0\right.$ in $\left.\mathrm{CH}_{2} \mathrm{Cl}_{2}\right) .{ }^{1} \mathbf{H}$ NMR $\left(500 \mathrm{MHz}, \mathrm{CDCl}_{3}\right) \delta 8.04-8.03(\mathrm{~m}, 2 \mathrm{H}), 7.52-7.48(\mathrm{~m}$, 5H), 7.31-7.28 (m, 2H), 7.26-7.22 (m, 3H), $7.12(\mathrm{t}, J=8.0 \mathrm{~Hz}, 1 \mathrm{H}), 6.92(\mathrm{t}, J=8.6 \mathrm{~Hz}, 2 \mathrm{H}), 6.81$ (dd, $J=8.2 \mathrm{~Hz}, 1.2 \mathrm{~Hz}, 1 \mathrm{H}), 6.62(\mathrm{~d}, J=7.7 \mathrm{~Hz}, 1 \mathrm{H}), 5.73(\mathrm{~s}, 1 \mathrm{H}), 5.42(\mathrm{~s}, 1 \mathrm{H}), 4.76(\mathrm{~s}, 1 \mathrm{H}), 3.81$ (s, 3H). ${ }^{13} \mathbf{C}$ NMR $\left(126 \mathrm{MHz}, \mathrm{CDCl}_{3}\right) \delta 200.8,161.5\left(\mathrm{~d},{ }^{1} J_{\mathrm{C}-\mathrm{F}}=243.9 \mathrm{~Hz}\right), 151.7,144.1,138.3(\mathrm{~d}$, $\left.{ }^{4} J_{\mathrm{C}-\mathrm{F}}=2.6 \mathrm{~Hz}\right), 137.4,134.9,130.5,130.1\left(\mathrm{~d},{ }^{3} J_{\mathrm{C}-\mathrm{F}}=7.7 \mathrm{~Hz}\right), 128.7,128.6,128.4,127.5,127.4$, 127.1, 120.0, $115.3\left(\mathrm{~d},{ }^{2} J_{\mathrm{C}-\mathrm{F}}=21.1 \mathrm{~Hz}\right), 110.5,67.2,55.6,43.9$. HRMS (ESI) $\mathrm{m} / \mathrm{z}:[\mathrm{M}+\mathrm{Na}]+\mathrm{Calcd}$ for $\mathrm{C}_{30} \mathrm{H}_{23} \mathrm{FO}_{3} \mathrm{Na}$ 473.1523; Found 473.1531.

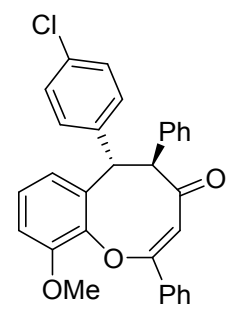


(5S,6S,Z)-6-(4-chlorophenyl)-10-methoxy-2,5-diphenyl-5,6-dihydro-4H-benzo[b]oxocin-4-

one (4pa). Purified by silica gel chromatography using PE/EA 4:1, white solid, mp 127-130 ${ }^{\circ} \mathrm{C}$, $78 \%$ yield, $36.3 \mathrm{mg}$, 95\% ee, $>20: 1 \mathrm{dr}$. The enantiomeric excess was determined by HPLC on Daicel Chiralpak IB-H with hexane/i-PrOH (90:10) as the eluent, flow $=1.0 \mathrm{~mL} / \mathrm{min}, \mathrm{UV}=254 \mathrm{~nm},[\alpha]_{D}^{25}$ $=-314\left(\mathrm{c}=1.0\right.$ in $\left.\mathrm{CH}_{2} \mathrm{Cl}_{2}\right) .{ }^{1} \mathbf{H}$ NMR $\left(500 \mathrm{MHz}, \mathrm{CDCl}_{3}\right) \delta 8.03-8.02(\mathrm{~m}, 2 \mathrm{H}), 7.52-7.48(\mathrm{~m}$, $5 \mathrm{H}), 7.31-7.28(\mathrm{~m}, 2 \mathrm{H}), 7.25-7.18(\mathrm{~m}, 5 \mathrm{H}), 7.11(\mathrm{t}, J=8.0 \mathrm{~Hz}, 1 \mathrm{H}), 6.81(\mathrm{dd}, J=8.1 \mathrm{~Hz}, 1.0 \mathrm{~Hz}$, $1 \mathrm{H}), 6.61(\mathrm{~d}, J=7.6 \mathrm{~Hz}, 1 \mathrm{H}), 5.72(\mathrm{~s}, 1 \mathrm{H}), 5.41(\mathrm{~s}, 1 \mathrm{H}), 4.76(\mathrm{~s}, 1 \mathrm{H}), 3.81(\mathrm{~s}, 3 \mathrm{H}) .{ }^{13} \mathbf{C}$ NMR $(126$ $\left.\mathrm{MHz}, \mathrm{CDCl}_{3}\right) \delta 200.7,162.2,151.8,144.1,138.6,138.0,137.3,134.8,132.5,130.5,130.0,128.7$, 128.6, 128.4 127.6, 127.4, 127.1, 120.2, 110.6, 105.6, 66.8, 55.6, 45.5. HRMS (ESI) m/z: [M + $\mathrm{Na}]+$ Calcd for $\mathrm{C}_{30} \mathrm{H}_{23} \mathrm{ClO}_{3} \mathrm{Na} 489.1228$; Found 489.1236.

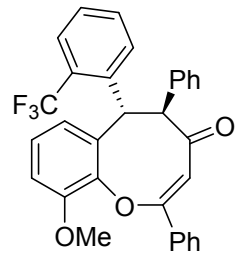

(5S,6S,Z)-10-methoxy-2,5-diphenyl-6-(2-(trifluoromethyl)phenyl)-5,6-dihydro-4H-

benzo[b]oxocin-4-one (4qa). Purified by silica gel chromatography using PE/EA 4:1, white solid, mp $161-163{ }^{\circ} \mathrm{C}, 87 \%$ yield, $43.5 \mathrm{mg}, 97 \%$ ee, $>20: 1 \mathrm{dr}$. The enantiomeric excess was determined by HPLC on Daicel Chiralpak IB-H with hexane/i-PrOH (90:10) as the eluent, flow $=1.0 \mathrm{~mL} / \mathrm{min}$, $\mathrm{UV}=254 \mathrm{~nm},[\alpha]_{D}^{25}=-344\left(\mathrm{c}=1.0\right.$ in $\left.\mathrm{CH}_{2} \mathrm{Cl}_{2}\right) .{ }^{1} \mathbf{H}$ NMR $\left(500 \mathrm{MHz}, \mathrm{CDCl}_{3}\right) \delta 8.04-8.02(\mathrm{~m}$, 2H), $7.79(\mathrm{~d}, J=7.9 \mathrm{~Hz}, 1 \mathrm{H}), 7.60(\mathrm{~d}, J=7.7 \mathrm{~Hz}, 1 \mathrm{H}), 7.55-7.48(\mathrm{~m}, 6 \mathrm{H}), 7.30-7.24(\mathrm{~m}, 3 \mathrm{H}), 7.19$ (t, $J=7.3 \mathrm{~Hz}, 1 \mathrm{H}), 7.11(\mathrm{t}, J=8.1 \mathrm{~Hz}, 1 \mathrm{H}), 6.81(\mathrm{dd}, J=8.2,1.1 \mathrm{~Hz}, 1 \mathrm{H}), 6.65(\mathrm{dd}, J=7.9,0.9 \mathrm{~Hz}$, $1 \mathrm{H}), 6.15(\mathrm{~d}, J=12.5 \mathrm{~Hz}, 1 \mathrm{H}), 5.59(\mathrm{~s}, 1 \mathrm{H}), 4.72(\mathrm{~d}, J=12.6 \mathrm{~Hz}, 1 \mathrm{H}), 3.84(\mathrm{~s}, 3 \mathrm{H}) .{ }^{13} \mathbf{C}$ NMR $(126$ $\left.\mathrm{MHz}, \mathrm{CDCl}_{3}\right) \delta 200.4,160.9,151.6,142.9,137.9,137.0,136.9,135.2,131.6,130.4,129.7\left(\mathrm{q},{ }^{2} J_{\mathrm{C}-\mathrm{F}}\right.$ $=29.5 \mathrm{~Hz}), 129.2,128.7,128.5,128.5,127.6,127.5,127.1,127.0,126.9\left(\mathrm{q},{ }^{3} J_{\mathrm{C}-\mathrm{F}}=6.0 \mathrm{~Hz}\right), 124.4$ $\left(\mathrm{q},{ }^{1} J_{\mathrm{C}-\mathrm{F}}=272.8 \mathrm{~Hz}\right), 118.3,110.5,103.3,68.7,55.7,38.6$. HRMS (ESI) $\mathrm{m} / \mathrm{z}:[\mathrm{M}+\mathrm{Na}]+$ Calcd for $\mathrm{C}_{31} \mathrm{H}_{23} \mathrm{~F}_{3} \mathrm{O}_{3} \mathrm{Na}$ 523.1492; Found 523.1502.

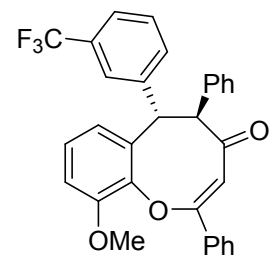

(5S,6S,Z)-10-methoxy-2,5-diphenyl-6-(3-(trifluoromethyl)phenyl)-5,6-dihydro-4H-

benzo[b]oxocin-4-one (4ra). Purified by silica gel chromatography using PE/EA 4:1, white solid, mp 102-104 ${ }^{\circ} \mathrm{C}, 68 \%$ yield, $34.0 \mathrm{mg}, 96 \%$ ee, $>20: 1 \mathrm{dr}$. The enantiomeric excess was determined by HPLC on Daicel Chiralpak IB-H with hexane/i-PrOH (90:10) as the eluent, flow $=1.0 \mathrm{~mL} / \mathrm{min}$, $\mathrm{UV}=254 \mathrm{~nm},[\alpha]_{D}^{25}=-254\left(\mathrm{c}=1.0\right.$ in $\left.\mathrm{CH}_{2} \mathrm{Cl}_{2}\right) .{ }^{1} \mathbf{H} \mathbf{~ N M R}\left(500 \mathrm{MHz}, \mathrm{CDCl}_{3}\right) \delta 8.04-8.03(\mathrm{~m}$, 2H), 7.52-7.47 (m, 7H), $7.42(\mathrm{~d}, J=7.7 \mathrm{~Hz}, 1 \mathrm{H}), 7.34(\mathrm{t}, J=7.7 \mathrm{~Hz}, 1 \mathrm{H}), 7.31-7.27(\mathrm{~m}, 2 \mathrm{H}), 7.24$ $(\mathrm{t}, J=7.3 \mathrm{~Hz}, 1 \mathrm{H}), 7.12(\mathrm{t}, J=8.0 \mathrm{~Hz}, 1 \mathrm{H}), 6.82(\mathrm{~d}, J=8.1 \mathrm{~Hz}, 1 \mathrm{H}), 6.58(\mathrm{~d}, J=7.3 \mathrm{~Hz}, 1 \mathrm{H}), 5.75$ (s, 1H), 5.49 (s, 1H), $4.80(\mathrm{~s}, 1 \mathrm{H}), 3.81(\mathrm{~s}, 3 \mathrm{H}) .{ }^{13} \mathbf{C} \mathbf{~ N M R}\left(126 \mathrm{MHz}, \mathrm{CDCl}_{3}\right) \delta$ 200.34, 151.87, $144.28,140.98,137.57,137.15,134.78,131.88,130.7$ (q, $\left.{ }^{2} J_{\mathrm{C}-\mathrm{F}}=31.9 \mathrm{~Hz}\right) 130.57,128.89,128.72$, $128.68,128.44,127.69,127.37,127.23,125.68,123.9$ (q, $\left.{ }^{1} J_{\mathrm{C}-\mathrm{F}}=270.7 \mathrm{~Hz}\right), 123.7$ (q, ${ }^{3} J_{\mathrm{C}-\mathrm{F}}=3.7$ $\mathrm{Hz}), 120.07,110.76,66.64,55.61,45.56$. HRMS (ESI) $\mathrm{m} / \mathrm{z}$ : $[\mathrm{M}+\mathrm{Na}]+$ Calcd for $\mathrm{C}_{31} \mathrm{H}_{23} \mathrm{~F}_{3} \mathrm{O}_{3} \mathrm{Na}$ 523.1492; Found 523.1505. 


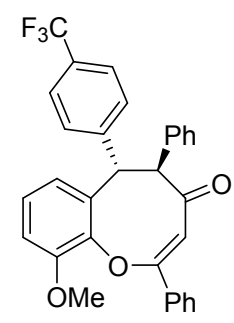

(5S,6S,Z)-10-methoxy-2,5-diphenyl-6-(4-(trifluoromethyl)phenyl)-5,6-dihydro-4H-

benzo[b]oxocin-4-one (4sa). Purified by silica gel chromatography using PE/EA 4:1, white solid, $\mathrm{mp} 118-120^{\circ} \mathrm{C}, 70 \%$ yield, $35.0 \mathrm{mg}, 96 \%$ ee, $>20: 1 \mathrm{dr}$. The enantiomeric excess was determined by HPLC on Daicel Chiralpak IB-H with hexane $/ \mathrm{i}-\mathrm{PrOH}(90: 10)$ as the eluent, flow $=1.0 \mathrm{~mL} / \mathrm{min}$, $\mathrm{UV}=254 \mathrm{~nm},[\alpha]_{D}^{25}=-208\left(\mathrm{c}=1.0\right.$ in $\left.\mathrm{CH}_{2} \mathrm{Cl}_{2}\right) .{ }^{1} \mathbf{H} \mathbf{~ N M R}\left(500 \mathrm{MHz}, \mathrm{CDCl}_{3}\right) \delta 8.04-8.02(\mathrm{~m}$, 2H), 7.51-7.48 (m, 7H), $7.39(\mathrm{~d}, J=8.1 \mathrm{~Hz}, 1 \mathrm{H}), 7.31-7.28(\mathrm{~m}, 2 \mathrm{H}), 7.24(\mathrm{t}, J=7.3 \mathrm{~Hz}, 1 \mathrm{H}), 7.12$ $(\mathrm{t}, J=8.0 \mathrm{~Hz}, 1 \mathrm{H}), 6.82(\mathrm{dd}, J=8.2 \mathrm{~Hz}, 0.8,1 \mathrm{H}), 6.58(\mathrm{~d}, J=7.2 \mathrm{~Hz}, 1 \mathrm{H}), 5.75(\mathrm{~s}, 1 \mathrm{H}), 5.47(\mathrm{~s}$, 1H), $4.82(\mathrm{~s}, 1 \mathrm{H}), 3.81(\mathrm{~s}, 3 \mathrm{H}) .{ }^{13} \mathrm{C}$ NMR $\left(126 \mathrm{MHz}, \mathrm{CDCl}_{3}\right) \delta 200.5,152.0,144.3,137.6,137.2$, $134.8,130.7,129.1,128.8,128.8\left(\mathrm{q},{ }^{2} J_{\mathrm{C}-\mathrm{F}}=27.3 \mathrm{~Hz}\right), 128.5,127.8,127.5,127.3,125.5\left(\mathrm{q},{ }^{3} J_{\mathrm{C}-\mathrm{F}}=\right.$ $3.0 \mathrm{~Hz}), 124.1\left(\mathrm{q},{ }^{1} J_{\mathrm{C}-\mathrm{F}}=225.3 \mathrm{~Hz}\right), 120.2,110.8,105.1,66.8,55.7,43.5$. HRMS (ESI) $\mathrm{m} / \mathrm{z}:[\mathrm{M}+$ $\mathrm{Na}]+$ Calcd for $\mathrm{C}_{31} \mathrm{H}_{23} \mathrm{~F}_{3} \mathrm{O}_{3} \mathrm{Na}$ 523.1492; Found 523.1509.<smiles>COc1cccc2c1O/C(c1ccccc1)=C/C(=O)C(c1ccccc1)C2c1ccccc1</smiles>

(5S,6S,Z)-10-methoxy-2,5-diphenyl-6-(3-(trifluoromethoxy)phenyl)-5,6-dihydro-4H-

benzo[b]oxocin-4-one (4ta). Purified by silica gel chromatography using PE/EA 4:1, white solid, $\mathrm{mp} 87-90{ }^{\circ} \mathrm{C}, 69 \%$ yield, $35.6 \mathrm{mg}, 95 \%$ ee, $>20: 1 \mathrm{dr}$. The enantiomeric excess was determined by HPLC on Daicel Chiralpak IB-H with hexane/i-PrOH (90:10) as the eluent, flow $=1.0 \mathrm{~mL} / \mathrm{min}, \mathrm{UV}$ $=254 \mathrm{~nm},[\alpha]_{D}^{25}=-233\left(\mathrm{c}=1.0\right.$ in $\left.\mathrm{CH}_{2} \mathrm{Cl}_{2}\right) .{ }^{1} \mathbf{H}$ NMR $\left(500 \mathrm{MHz}, \mathrm{CDCl}_{3}\right) \delta$ 8.04-8.02 (m, 2H), 7.52-7.48 (m, 5H), 7.31-7.28 (m, 2H), 7.25-7.20 (m, 3H), 7.14-7.11 (m, 2H), 7.01 (d, $J=7.8 \mathrm{~Hz}$, $1 \mathrm{H}), 6.82(\mathrm{~d}, J=8.1 \mathrm{~Hz}, 1 \mathrm{H}), 6.61(\mathrm{~d}, J=7.6 \mathrm{~Hz}, 1 \mathrm{H}), 5.74(\mathrm{~s}, 1 \mathrm{H}), 5.42(\mathrm{~s}, 1 \mathrm{H}), 4.76(\mathrm{~s}, 1 \mathrm{H}), 3.81$ (s, 3H). ${ }^{13} \mathbf{C}$ NMR $\left(126 \mathrm{MHz}, \mathrm{CDCl}_{3}\right) \delta 200.5,151.8,149.2,144.2,142.5,137.6,137.2,134.8,130.6$, 129.7, 128.7, 128.4, 127.6, 127.4, 127.2, 127.1, 121.3, 120.3 (d, $\left.{ }^{1} J_{\mathrm{C}-\mathrm{F}}=255.6 \mathrm{~Hz}\right), 120.1,119.1$, 110.7, 66.8, 55.6, 44.8. HRMS (ESI) m/z: [M + Na] + Calcd for $\mathrm{C}_{31} \mathrm{H}_{23} \mathrm{~F}_{3} \mathrm{O}_{4} \mathrm{Na}$ 539.1441; Found 539.1456 .

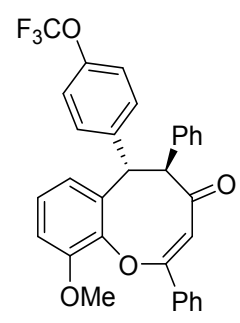

(5S,6S,Z)-10-methoxy-2,5-diphenyl-6-(4-(trifluoromethoxy)phenyl)-5,6-dihydro-4H-

benzo[b]oxocin-4-one (4ua). Purified by silica gel chromatography using PE/EA 4:1, white solid, $\mathrm{mp} 115-118^{\circ} \mathrm{C}, 73 \%$ yield, $37.6 \mathrm{mg}, 97 \%$ ee, $>20: 1 \mathrm{dr}$. The enantiomeric excess was determined by HPLC on Daicel Chiralpak IB-H with hexane/i-PrOH $(90: 10)$ as the eluent, flow $=1.0 \mathrm{~mL} / \mathrm{min}$, $\mathrm{UV}=254 \mathrm{~nm},[\alpha]_{D}^{25}=-202\left(\mathrm{c}=1.0\right.$ in $\left.\mathrm{CH}_{2} \mathrm{Cl}_{2}\right) .{ }^{1} \mathbf{H} \mathbf{~ N M R}\left(500 \mathrm{MHz}, \mathrm{CDCl}_{3}\right) \delta 8.03(\mathrm{dd}, J=$ 
7.5, 1.7 Hz, 2H), 7.51-7.48 (m, 6H), 7.32-7.28 (m, 4H), $7.25(\mathrm{dt}, J=8.5,1.9 \mathrm{~Hz}, 1 \mathrm{H}), 7.12(\mathrm{t}, J=$ $8.0 \mathrm{~Hz}, 1 \mathrm{H}), 7.07(\mathrm{~d}, J=8.2 \mathrm{~Hz}, 1 \mathrm{H}), 6.82(\mathrm{dd}, J=8.2,1.2 \mathrm{~Hz}, 1 \mathrm{H}), 6.61(\mathrm{~d}, J=7.4 \mathrm{~Hz}, 1 \mathrm{H}), 5.75$ (s, 1H), 5.43 (s, 1H), 4.79 (s, 1H), 3.81 (s, 3H). ${ }^{13} \mathbf{C}$ NMR (126 MHz, $\left.\mathrm{CDCl}_{3}\right) \delta 200.5,151.8,147.9$, 144.3, 138.7, 137.9, 137.3, 134.8, 130.5, 130.0, 128.7, 128.4, 127.6, 127.4, 127.2, 120.8, 120.4 (q, $J=257.1 \mathrm{~Hz}), 120.2,110.6,105.9,67.1,55.6,45.5$. HRMS (ESI) $\mathrm{m} / \mathrm{z}:[\mathrm{M}+\mathrm{Na}]+$ Calcd for $\mathrm{C}_{31} \mathrm{H}_{23} \mathrm{~F}_{3} \mathrm{O}_{4} \mathrm{Na}$ 539.1441; Found 539.1455.

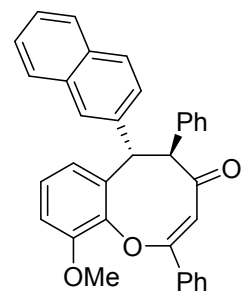

(5S,6S,Z)-10-methoxy-6-(naphthalen-2-yl)-2,5-diphenyl-5,6-dihydro-4H-benzo[b]oxocin-4one (4va). Purified by silica gel chromatography using PE/EA 4:1, white solid, mp $255-257^{\circ} \mathrm{C}, 72 \%$ yield, $34.7 \mathrm{mg}, 94 \% e e,>20: 1 \mathrm{dr}$. The enantiomeric excess was determined by HPLC on Daicel Chiralpak IB-H with hexane/i-PrOH (90:10) as the eluent, flow $=1.0 \mathrm{~mL} / \mathrm{min}, \mathrm{UV}=254 \mathrm{~nm},[\alpha]_{D}^{25}$ $=-267\left(\mathrm{c}=1.0\right.$ in $\left.\mathrm{CH}_{2} \mathrm{Cl}_{2}\right) .{ }^{1} \mathbf{H}$ NMR $\left(500 \mathrm{MHz}, \mathrm{CDCl}_{3}\right) \delta 8.07-8.05(\mathrm{~m}, 2 \mathrm{H}), 7.77-7.73(\mathrm{~m}, 4 \mathrm{H})$, $7.58(\mathrm{~d}, J=7.5 \mathrm{~Hz}, 2 \mathrm{H}), 7.53-7.51(\mathrm{~m}, 3 \mathrm{H}), 7.44-7.42(\mathrm{~m}, 3 \mathrm{H}), 7.29-7.26(\mathrm{~m}, 2 \mathrm{H}), 7.20(\mathrm{t}, J=7.3$ Hz, 1H), 7.09 (t, $J=8.0 \mathrm{~Hz}, 1 \mathrm{H}), 6.80$ (d, $J=8.2 \mathrm{~Hz}, 1 \mathrm{H}), 6.67$ (d, $J=7.7 \mathrm{~Hz}, 1 \mathrm{H}), 5.72(\mathrm{~s}, 2 \mathrm{H})$, $4.93(\mathrm{~s}, 1 \mathrm{H}), 3.84(\mathrm{~s}, 3 \mathrm{H}) .{ }^{13} \mathbf{C}$ NMR $\left(126 \mathrm{MHz}, \mathrm{CDCl}_{3}\right) \delta 200.9,151.6,138.5,137.5,135.1,133.3$, 132.3, 130.5, 128.7, 128.5, 128.2, 127.8, 127.5, 127.5, 127.3, 127.1, 126.0, 125.7, 120.3, 110.4, 67.3, 55.7, 43.7. HRMS (ESI) m/z: [M + Na]+ Calcd for $\mathrm{C}_{34} \mathrm{H}_{26} \mathrm{O}_{3} \mathrm{Na}$ 505.1774; Found 505.1789.<smiles></smiles>

(5S,6R,Z)-6-(furan-2-yl)-10-methoxy-2,5-diphenyl-5,6-dihydro-4H-benzo[b]oxocin-4-one

(4wa). Purified by silica gel chromatography using PE/EA 4:1, white solid, mp 173-175 ${ }^{\circ} \mathrm{C}, 65 \%$ yield, $27.4 \mathrm{mg}, 98 \%$ ee, $>20: 1 \mathrm{dr}$. The enantiomeric excess was determined by HPLC on Daicel Chiralpak IC-H with hexane/i-PrOH (90:10) as the eluent, flow $=1.0 \mathrm{~mL} / \mathrm{min}, \mathrm{UV}=254 \mathrm{~nm},[\alpha]_{D}^{25}$ $=-231\left(\mathrm{c}=1.0\right.$ in $\left.\mathrm{CH}_{2} \mathrm{Cl}_{2}\right) .{ }^{1} \mathbf{H}$ NMR $\left(500 \mathrm{MHz}, \mathrm{CDCl}_{3}\right) \delta 8.03-8.01(\mathrm{~m}, 2 \mathrm{H}), 7.55-7.49(\mathrm{~m}, 5 \mathrm{H})$, 7.35-7.31 (m, 3H), 7.29-7.26 (m, 1H), $7.20(\mathrm{t}, J=8.0 \mathrm{~Hz}, 1 \mathrm{H}), 6.86(\mathrm{dd}, J=8.2,1.2 \mathrm{~Hz}, 1 \mathrm{H}), 6.81$ (dd, $J=7.9,1.2 \mathrm{~Hz}, 1 \mathrm{H}), 6.24(\mathrm{dd}, J=3.2,1.9 \mathrm{~Hz}, 1 \mathrm{H}), 6.12(\mathrm{~d}, J=3.3 \mathrm{~Hz}, 1 \mathrm{H}), 5.64(\mathrm{~d}, J=12.6$ $\mathrm{Hz}, 1 \mathrm{H}), 5.59(\mathrm{~s}, 1 \mathrm{H}), 4.50(\mathrm{~d}, J=12.6 \mathrm{~Hz}, 1 \mathrm{H}), 3.87(\mathrm{~s}, 3 \mathrm{H}) .{ }^{13} \mathbf{C} \mathbf{N M R}\left(126 \mathrm{MHz}, \mathrm{CDCl}_{3}\right) \delta 199.6$, 160.66, 152.6, 151.5, 143.3, 141.9, 137.4, 136.5, 135.2, 130.5, 128.7, 128.5, 128.2, 127.7, 127.6, 127.4, 118.8, 110.8, 110.1, 108.2, 103.9, 68.2, 55.8, 38.4. HRMS (ESI) m/z: [M + Na]+ Calcd for $\mathrm{C}_{28} \mathrm{H}_{22} \mathrm{O}_{4} \mathrm{Na}$ 445.1410; Found 445.1424.<smiles>CC[C@H]1c2cccc(OC)c2OC(c2ccccc2)CC(=O)[C@H]1c1ccccc1</smiles>

(5S,6R,Z)-6-ethyl-10-methoxy-2,5-diphenyl-5,6-dihydro-4H-benzo[b]oxocin-4-one (4xa). 
Purified by silica gel chromatography using PE/EA 4:1, white solid, mp $117-120{ }^{\circ} \mathrm{C}, 30 \%$ yield, $11.5 \mathrm{mg}, 96 \% e e,>20: 1 \mathrm{dr}$. The enantiomeric excess was determined by HPLC on Daicel Chiralpak IB-H with hexane/i-PrOH (90:10) as the eluent, flow $=1.0 \mathrm{~mL} / \mathrm{min}, \mathrm{UV}=254 \mathrm{~nm},[\alpha]_{D}^{25}=-225$ $\left(\mathrm{c}=1.0\right.$ in $\left.\mathrm{CH}_{2} \mathrm{Cl}_{2}\right) .{ }^{1} \mathbf{H}$ NMR $\left(600 \mathrm{MHz}, \mathrm{CDCl}_{3}\right) \delta 8.03(\mathrm{~d}, J=7.7 \mathrm{~Hz}, 2 \mathrm{H}), 7.54(\mathrm{~d}, J=7.7 \mathrm{~Hz}$, 2H), 7.51-1.48 (m, 3H), 7.40 (t, $J=7.6 \mathrm{~Hz}, 2 \mathrm{H}), 7.33(\mathrm{t}, J=7.3 \mathrm{~Hz}, 1 \mathrm{H}), 7.28(\mathrm{~s}, 1 \mathrm{H}), 6.97$ (d, $J=$ $5.9 \mathrm{~Hz}, 1 \mathrm{H}), 6.86(\mathrm{~d}, J=8.1 \mathrm{~Hz}, 1 \mathrm{H}), 5.54(\mathrm{~s}, 1 \mathrm{H}), 4.10(\mathrm{~s}, 1 \mathrm{H}), 3.87(\mathrm{~s}, 3 \mathrm{H}), 3.72(\mathrm{~s}, 1 \mathrm{H}), 1.76-$ $1.64(\mathrm{~m}, 2 \mathrm{H}), 0.82(\mathrm{t}, J=7.3 \mathrm{~Hz}, 3 \mathrm{H}) .{ }^{13} \mathbf{C}$ NMR $\left(126 \mathrm{MHz}, \mathrm{CDCl}_{3}\right) \delta 200.9,151.6,138.4,137.5$, 135.4, 130.4, 128.8, 128.6, 128.4, 127.6, 127.2, 116.8, 110.2, 103.7, 72.7, 55.6, 53.4, 39.7, 11.9. HRMS (ESI) m/z: [M + Na]+ Calcd for $\mathrm{C}_{26} \mathrm{H}_{24} \mathrm{O}_{3} \mathrm{Na}$ 407.1618; Found 407.1625.

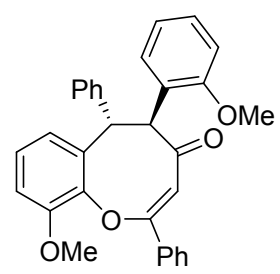

(5S,6S,Z)-10-methoxy-5-(2-methoxyphenyl)-2,6-diphenyl-5,6-dihydro-4H-benzo[b]oxocin-4one (4ab). Purified by silica gel chromatography using PE/EA 4:1, white solid, mp 202-204 ${ }^{\circ} \mathrm{C}$, $73 \%$ yield, $33.7 \mathrm{mg}, 93 \% e e,>20: 1 \mathrm{dr}$. The enantiomeric excess was determined by HPLC on Daicel Chiralpak IB-H with hexane/i-PrOH (90:10) as the eluent, flow $=1.0 \mathrm{~mL} / \mathrm{min}, \mathrm{UV}=254 \mathrm{~nm},[\alpha]_{D}^{25}$ $=-277\left(\mathrm{c}=1.0\right.$ in $\left.\mathrm{CH}_{2} \mathrm{Cl}_{2}\right) .{ }^{1} \mathrm{H}$ NMR $\left(600 \mathrm{MHz}, \mathrm{CDCl}_{3}\right) \delta 8.09(\mathrm{dd}, J=6.1,2.5 \mathrm{~Hz}, 2 \mathrm{H}), 7.77$ (d, $J=7.6 \mathrm{~Hz}, 1 \mathrm{H}), 7.53-7.52(\mathrm{~m}, 3 \mathrm{H}), 7.38(\mathrm{~d}, J=7.3 \mathrm{~Hz}, 2 \mathrm{H}), 7.24$ (t, $J=7.6 \mathrm{~Hz}, 2 \mathrm{H}), 7.20$ (td, $J$ $=8.2,1.6 \mathrm{~Hz}, 1 \mathrm{H}), 7.15(\mathrm{t}, J=7.4 \mathrm{~Hz}, 1 \mathrm{H}), 7.11(\mathrm{t}, J=8.0 \mathrm{~Hz}, 1 \mathrm{H}), 6.95(\mathrm{t}, J=7.5 \mathrm{~Hz}, 1 \mathrm{H}), 6.86$ $(\mathrm{d}, J=8.2 \mathrm{~Hz}, 1 \mathrm{H}), 6.79(\mathrm{dd}, J=8.2,1.1 \mathrm{~Hz}, 1 \mathrm{H}), 6.67(\mathrm{~d}, J=7.8 \mathrm{~Hz}, 1 \mathrm{H}), 5.76(\mathrm{~s}, 1 \mathrm{H}), 5.50(\mathrm{~s}$, $1 \mathrm{H}), 5.43(\mathrm{~s}, 1 \mathrm{H}), 3.87(\mathrm{~s}, 3 \mathrm{H}), 3.82(\mathrm{~s}, 3 \mathrm{H}) .{ }^{13} \mathrm{C}$ NMR $\left(126 \mathrm{MHz}, \mathrm{CDCl}_{3}\right) \delta 200.9,157.4,151.6$, 140.2, 138.9, 135.4, 130.3, 128.6, 128.4, 128.4, 128.3, 127.4, 127.0, 126.6, 126.5, 120.7, 120.3, 111.3, 110.2, 106.7, 68.0, 55.9, 55.6, 48.5. HRMS (ESI) m/z: $[\mathrm{M}+\mathrm{Na}]+$ Calcd for $\mathrm{C}_{31} \mathrm{H}_{26} \mathrm{O}_{4} \mathrm{Na}$ 485.1723; Found 485.1734.

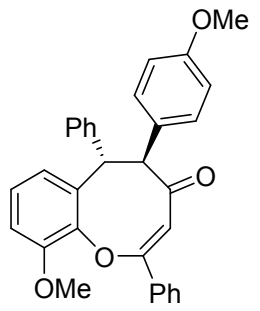

(5S,6S,Z)-10-methoxy-5-(4-methoxyphenyl)-2,6-diphenyl-5,6-dihydro-4H-benzo[b]oxocin-4one (4ac). Purified by silica gel chromatography using PE/EA 4:1, white solid, mp 108-110 ${ }^{\circ} \mathrm{C}, 71 \%$ yield, $32.8 \mathrm{mg}, 96 \% e e,>20: 1 \mathrm{dr}$. The enantiomeric excess was determined by HPLC on Daicel Chiralpak IB-H with hexane/i-PrOH (90:10) as the eluent, flow $=1.0 \mathrm{~mL} / \mathrm{min}, \mathrm{UV}=254 \mathrm{~nm},[\alpha]_{D}^{25}$ $=-237\left(\mathrm{c}=1.0\right.$ in $\left.\mathrm{CH}_{2} \mathrm{Cl}_{2}\right) .{ }^{1} \mathbf{H}$ NMR $\left(500 \mathrm{MHz}, \mathrm{CDCl}_{3}\right) \delta 8.06(\mathrm{~d}, J=4.3 \mathrm{~Hz}, 2 \mathrm{H}), 7.52-7.51$ (m, 3H), $7.45(\mathrm{~d}, J=8.3 \mathrm{~Hz}, 2 \mathrm{H}), 7.32(\mathrm{~d}, J=7.6 \mathrm{~Hz}, 2 \mathrm{H}), 7.26(\mathrm{t}, J=7.5 \mathrm{~Hz}, 2 \mathrm{H}), 7.18(\mathrm{t}, J=7.2$ $\mathrm{Hz}, 1 \mathrm{H}), 7.11$ (t, $J=8.0 \mathrm{~Hz}, 1 \mathrm{H}), 6.84(\mathrm{~d}, J=8.7 \mathrm{~Hz}, 2 \mathrm{H}), 6.80(\mathrm{~d}, J=7.4 \mathrm{~Hz}, 1 \mathrm{H}), 6.66(\mathrm{~d}, J=7.7$ $\mathrm{Hz}, 1 \mathrm{H}), 5.71(\mathrm{~s}, 1 \mathrm{H}), 5.49$ (s, 1H), $4.75(\mathrm{~s}, 1 \mathrm{H}), 3.82(\mathrm{~s}, 3 \mathrm{H}), 3.77$ (s, 3H). ${ }^{13} \mathbf{C}$ NMR $(126 \mathrm{MHz}$, $\left.\mathrm{CDCl}_{3}\right) \delta 201.1,161.5,158.8,151.6,143.9,139.8,138.6,135.0,130.4,129.6,128.8,128.4,128.4$, 127.4, 127.0, 126.6, 120.0, 114.0, 110.3, 104.9, 66.4, 55.6, 55.1, 43.2. HRMS (ESI) m/z: [M + Na]+ Calcd for $\mathrm{C}_{31} \mathrm{H}_{26} \mathrm{O}_{4} \mathrm{Na} 485.1723$; Found 485.1739 . 


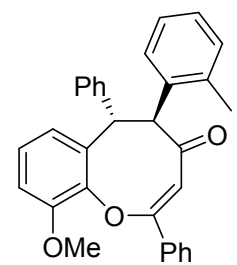

(5S,6S,Z)-10-methoxy-2,6-diphenyl-5-(o-tolyl)-5,6-dihydro-4H-benzo[b]oxocin-4-one (4ad). Purified by silica gel chromatography using PE/EA 4:1, white solid, mp 100-103 ${ }^{\circ} \mathrm{C}, 80 \%$ yield, $35.6 \mathrm{mg}, 98 \%$ ee, $>20: 1 \mathrm{dr}$. The enantiomeric excess was determined by HPLC on Daicel Chiralpak IB-H with hexane/i-PrOH $(90: 10)$ as the eluent, flow $=1.0 \mathrm{~mL} / \mathrm{min}, \mathrm{UV}=254 \mathrm{~nm},[\alpha]_{D}^{25}=-287$ $\left(\mathrm{c}=1.0\right.$ in $\left.\mathrm{CH}_{2} \mathrm{Cl}_{2}\right) .{ }^{1} \mathbf{H}$ NMR $\left(500 \mathrm{MHz}, \mathrm{CDCl}_{3}\right) \delta 8.03-8.02(\mathrm{~m}, 2 \mathrm{H}), 7.78(\mathrm{~d}, J=6.6 \mathrm{~Hz}, 1 \mathrm{H})$, 7.52-7.48 (m, 3H), 7.26-7.19 (m, 5H), 7.17-7.11 (m, 4H), $6.81(\mathrm{dd}, J=8.2,1.1 \mathrm{~Hz}, 1 \mathrm{H}), 6.67(\mathrm{~d}, J$ $=7.7 \mathrm{~Hz}, 1 \mathrm{H}), 5.63(\mathrm{~s}, 2 \mathrm{H}), 5.05(\mathrm{~s}, 1 \mathrm{H}), 3.84(\mathrm{~s}, 3 \mathrm{H}), 2.48(\mathrm{~s}, 3 \mathrm{H}) .{ }^{13} \mathbf{C}$ NMR $(126 \mathrm{MHz}, \mathrm{CDCl} 3)$ $\delta 200.5,151.6,143.7,139.8,138.6,137.3,135.7,135.2,130.7,130.3,128.6,128.4,127.4,127.1$, 126.7, 126.2, 120.1, 110.3, 105.2, 62.2, 55.7, 43.9, 20.3. HRMS (ESI) m/z: [M + Na]+ Calcd for $\mathrm{C}_{31} \mathrm{H}_{26} \mathrm{O}_{3} \mathrm{Na} 469.1774$; Found 469.1789.

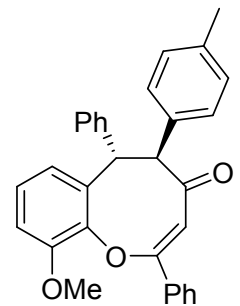

(5S,6S,Z)-10-methoxy-2,6-diphenyl-5-(p-tolyl)-5,6-dihydro-4H-benzo[b]oxocin-4-one (4ae). Purified by silica gel chromatography using PE/EA 4:1, white solid, mp 132-134 ${ }^{\circ} \mathrm{C}, 84 \%$ yield, $37.4 \mathrm{mg}, 97 \%$ ee, $>20: 1 \mathrm{dr}$. The enantiomeric excess was determined by HPLC on Daicel Chiralpak IB-H with hexane/i-PrOH (90:10) as the eluent, flow $=1.0 \mathrm{~mL} / \mathrm{min}, \mathrm{UV}=254 \mathrm{~nm},[\alpha]_{D}^{25}=-304$ $\left(\mathrm{c}=1.0\right.$ in $\left.\mathrm{CH}_{2} \mathrm{Cl}_{2}\right) .{ }^{1} \mathbf{H}$ NMR $\left(500 \mathrm{MHz}, \mathrm{CDCl}_{3}\right) \delta 8.06(\mathrm{~d}, J=4.7 \mathrm{~Hz}, 2 \mathrm{H}), 7.56-7.48(\mathrm{~m}, 3 \mathrm{H})$, $7.43(\mathrm{~d}, J=7.6 \mathrm{~Hz}, 2 \mathrm{H}), 7.33(\mathrm{~d}, J=7.5 \mathrm{~Hz}, 2 \mathrm{H}), 7.28-7.23(\mathrm{~m}, 2 \mathrm{H}), 7.18(\mathrm{t}, J=7.3 \mathrm{~Hz}, 1 \mathrm{H}), 7.13-$ $7.10(\mathrm{~m}, 3 \mathrm{H}), 6.81(\mathrm{~d}, J=7.9 \mathrm{~Hz}, 1 \mathrm{H}), 6.66(\mathrm{~d}, J=7.6 \mathrm{~Hz}, 1 \mathrm{H}), 5.70(\mathrm{~s}, 1 \mathrm{H}), 5.55(\mathrm{~s}, 1 \mathrm{H}), 4.77(\mathrm{~s}$, 1H), 3.83 (s, 3H), 2.32 (s, 3H). ${ }^{13} \mathbf{C}$ NMR (126 MHz, $\left.\mathrm{CDCl}_{3}\right) \delta 200.9,161.3,151.6,143.8,139.7$, $138.7,137.0,135.1,134.5,130.3,129.3,128.8,128.5,128.4,128.4,127.4,127.0,126.6,120.1$, 110.3, 105.2, 67.0, 55.6, 43.9, 21.0. HRMS (ESI) m/z: $[\mathrm{M}+\mathrm{Na}]+$ Calcd for $\mathrm{C}_{31} \mathrm{H}_{26} \mathrm{O}_{3} \mathrm{Na} 469.1774$; Found 469.1791.

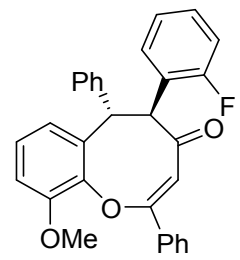

(5S,6S,Z)-5-(2-fluorophenyl)-10-methoxy-2,6-diphenyl-5,6-dihydro-4H-benzo[b]oxocin-4-one (4af). Purified by silica gel chromatography using PE/EA 4:1, white solid, mp 191-193 ${ }^{\circ} \mathrm{C}, 75 \%$ yield, $33.7 \mathrm{mg}, 98 \%$ ee, $>20: 1 \mathrm{dr}$. The enantiomeric excess was determined by HPLC on Daicel Chiralpak IB-H with hexane/i-PrOH (90:10) as the eluent, flow $=1.0 \mathrm{~mL} / \mathrm{min}, \mathrm{UV}=254 \mathrm{~nm},[\alpha]_{D}^{25}$ $=-219\left(\mathrm{c}=1.0\right.$ in $\left.\mathrm{CH}_{2} \mathrm{Cl}_{2}\right) .{ }^{1} \mathbf{H}$ NMR $\left(500 \mathrm{MHz}, \mathrm{CDCl}_{3}\right) \delta 8.11-8.09(\mathrm{~m}, 1 \mathrm{H}), 7.83(\mathrm{t}, J=6.6$ $\mathrm{Hz}, 1 \mathrm{H}), 7.54-7.53(\mathrm{~m}, 3 \mathrm{H}), 7.37$ (d, $J=7.5 \mathrm{~Hz}, 2 \mathrm{H}), 7.26(\mathrm{t}, J=7.6 \mathrm{~Hz}, 2 \mathrm{H}), 7.22-7.18(\mathrm{~m}, 2 \mathrm{H})$, 7.16-7.11 (m, 2H), $7.00(\mathrm{t}, J=9.1 \mathrm{~Hz}, 1 \mathrm{H}), 6.80(\mathrm{dd}, J=8.2,1.1 \mathrm{~Hz}, 1 \mathrm{H}), 6.72(\mathrm{~d}, J=7.8 \mathrm{~Hz}, 1 \mathrm{H})$, 
$5.88(\mathrm{~s}, 1 \mathrm{H}), 5.37(\mathrm{~s}, 2 \mathrm{H}), 3.78(\mathrm{~s}, 3 \mathrm{H}) .{ }^{13} \mathrm{C}$ NMR $\left(126 \mathrm{MHz}, \mathrm{CDCl}_{3}\right) \delta 199.9,163.2,160.7\left(\mathrm{~d},{ }^{1} J_{\mathrm{C}-}\right.$ $\left.\mathrm{F}_{\mathrm{F}}=245.3 \mathrm{~Hz}\right), 151.8,144.5,139.9,137.9,134.8,130.5,129.4,128.9\left(\mathrm{~d},{ }^{2} J_{\mathrm{C}-\mathrm{F}}=20.8 \mathrm{~Hz}\right), 128.5$, 128.4, 128.3, 127.4, 127.0, 126.8, $125.1\left(\mathrm{~d},{ }^{3} J_{\mathrm{C}-\mathrm{F}}=14.2 \mathrm{~Hz}\right), 124.1\left(\mathrm{~d},{ }^{4} J_{\mathrm{C}-\mathrm{F}}=3.3 \mathrm{~Hz}\right), 120.5,115.5$ $\left(\mathrm{d},{ }^{2} J_{\mathrm{C}-\mathrm{F}}=23.0 \mathrm{~Hz}\right), 110.5,107.1,57.4,55.5,46.3$. HRMS (ESI) $\mathrm{m} / \mathrm{z}:[\mathrm{M}+\mathrm{Na}]+$ Calcd for $\mathrm{C}_{30} \mathrm{H}_{23} \mathrm{FO}_{3} \mathrm{Na}$ 473.1523; Found 473.1542.

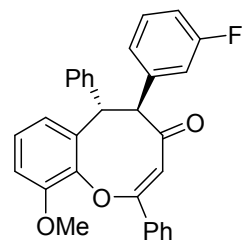

(5S,6S,Z)-5-(3-fluorophenyl)-10-methoxy-2,6-diphenyl-5,6-dihydro-4H-benzo[b]oxocin-4-one (4ag). Purified by silica gel chromatography using PE/EA 4:1, white solid, mp 213-215 ${ }^{\circ} \mathrm{C}, 70 \%$ yield, $31.5 \mathrm{mg}, 98 \%$ ee, $>20: 1 \mathrm{dr}$. The enantiomeric excess was determined by HPLC on Daicel Chiralpak IB-H with hexane/i-PrOH (90:10) as the eluent, flow $=1.0 \mathrm{~mL} / \mathrm{min}, \mathrm{UV}=254 \mathrm{~nm},[\alpha]_{D}^{25}$ $=-177\left(\mathrm{c}=1.0\right.$ in $\left.\mathrm{CH}_{2} \mathrm{Cl}_{2}\right) .{ }^{1} \mathbf{H}$ NMR $\left(500 \mathrm{MHz}, \mathrm{CDCl}_{3}\right) \delta 8.10-8.02(\mathrm{~m}, 2 \mathrm{H}), 7.54-7.49(\mathrm{~m}, 3 \mathrm{H})$, 7.30-7.22 (m, 7H), $7.18(\mathrm{t}, J=7.2 \mathrm{~Hz}, 1 \mathrm{H}), 7.11(\mathrm{t}, J=8.0 \mathrm{~Hz}, 1 \mathrm{H}), 6.94-6.90(\mathrm{~m}, 1 \mathrm{H}), 6.80(\mathrm{dd}, J$ $=8.2,0.9 \mathrm{~Hz}, 1 \mathrm{H}), 6.67(\mathrm{~d}, J=7.6 \mathrm{~Hz}, 1 \mathrm{H}), 5.78(\mathrm{~s}, 1 \mathrm{H}), 5.34(\mathrm{~s}, 1 \mathrm{H}), 4.86(\mathrm{~s}, 1 \mathrm{H}), 3.79(\mathrm{~s}, 3 \mathrm{H})$. ${ }^{13} \mathbf{C}$ NMR $\left(126 \mathrm{MHz}, \mathrm{CDCl}_{3}\right) \delta 200.3,162.7\left(\mathrm{~d},{ }^{1} J_{\mathrm{C}-\mathrm{F}}=244.6 \mathrm{~Hz}\right), 151.7,144.2,140.2\left(\mathrm{~d},{ }^{3} J_{\mathrm{C}-\mathrm{F}}=\right.$ $6.1 \mathrm{~Hz}), 139.8,137.9,134.7,130.5,129.8\left(\mathrm{~d},{ }^{3} J_{\mathrm{C}-\mathrm{F}}=7.8 \mathrm{~Hz}\right), 128.5,128.5,128.4,127.3,127.0$, $126.8,124.5,120.3,115.6\left(\mathrm{~d},{ }^{2} J_{\mathrm{C}-\mathrm{F}}=21.7 \mathrm{~Hz}\right), 114.3\left(\mathrm{~d},{ }^{2} J_{\mathrm{C}-\mathrm{F}}=20.9 \mathrm{~Hz}\right), 110.4,105.6,66.3,55.5$, 53.4. HRMS (ESI) m/z: $[\mathrm{M}+\mathrm{Na}]+$ Calcd for $\mathrm{C}_{30} \mathrm{H}_{23} \mathrm{FO}_{3} \mathrm{Na}$ 473.1523; Found 473.1540.

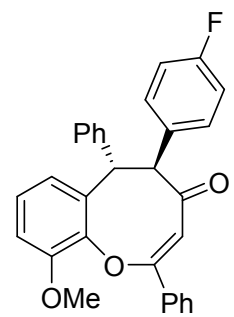

(5S,6S,Z)-5-(4-fluorophenyl)-10-methoxy-2,6-diphenyl-5,6-dihydro-4H-benzo[b]oxocin-4-one (4ah). Purified by silica gel chromatography using PE/EA 4:1, white solid, mp $210-212^{\circ} \mathrm{C}, 77 \%$ yield, $34.6 \mathrm{mg}, 96 \%$ ee, $>20: 1 \mathrm{dr}$. The enantiomeric excess was determined by HPLC on Daicel Chiralpak IB-H with hexane/i-PrOH (90:10) as the eluent, flow $=1.0 \mathrm{~mL} / \mathrm{min}, \mathrm{UV}=254 \mathrm{~nm},[\alpha]_{D}^{25}$ $=-247.000\left(\mathrm{c}=1.0\right.$ in $\left.\mathrm{CH}_{2} \mathrm{Cl}_{2}\right) .{ }^{1} \mathbf{H}$ NMR $\left(500 \mathrm{MHz}, \mathrm{CDCl}_{3}\right) \delta 8.07-8.06(\mathrm{~m}, 2 \mathrm{H}), 7.53-7.47(\mathrm{~m}$, $5 \mathrm{H}), 7.30-7.28(\mathrm{~m}, 2 \mathrm{H}), 7.25(\mathrm{t}, J=7.3 \mathrm{~Hz}, 2 \mathrm{H}), 7.18(\mathrm{t}, J=7.1 \mathrm{~Hz}, 1 \mathrm{H}), 7.11(\mathrm{t}, J=8.0 \mathrm{~Hz}, 1 \mathrm{H})$, $6.98(\mathrm{t}, J=8.5 \mathrm{~Hz}, 2 \mathrm{H}), 6.80(\mathrm{~d}, J=8.1 \mathrm{~Hz}, 1 \mathrm{H}), 6.68(\mathrm{~d}, J=7.4 \mathrm{~Hz}, 1 \mathrm{H}), 5.79(\mathrm{~s}, 1 \mathrm{H}), 5.34(\mathrm{~s}$, $1 \mathrm{H}), 4.87(\mathrm{~s}, 1 \mathrm{H}), 3.80(\mathrm{~s}, 3 \mathrm{H}){ }^{13} \mathrm{C}$ NMR $\left(126 \mathrm{MHz}, \mathrm{CDCl}_{3}\right) \delta 201.0,162.1\left(\mathrm{~d},{ }^{1} J_{\mathrm{C}-\mathrm{F}}=244.6 \mathrm{~Hz}\right)$, $151.8\left(\mathrm{~d},{ }^{4} J_{\mathrm{C}-\mathrm{F}}=3.7 \mathrm{~Hz}\right), 144.3,139.9,138.1,134.8,133.5,130.6,130.3\left(\mathrm{~d},{ }^{3} J_{\mathrm{C}-\mathrm{F}}=7.3 \mathrm{~Hz}\right), 128.6$, $128.5,128.4,127.3,127.0,126.8,120.4,115.4\left(\mathrm{~d},{ }^{2} J_{\mathrm{C}-\mathrm{F}}=21.1 \mathrm{~Hz}\right), 110.4,65.9,55.6,44.8$. HRMS (ESI) $\mathrm{m} / \mathrm{z}:[\mathrm{M}+\mathrm{Na}]+$ Calcd for $\mathrm{C}_{30} \mathrm{H}_{23} \mathrm{FO}_{3} \mathrm{Na} 473.1523$; Found 473.1538 .<smiles></smiles> 
(5S,6S,Z)-5-(2-chlorophenyl)-10-methoxy-2,6-diphenyl-5,6-dihydro-4H-benzo[b]oxocin-4one (4ai). Purified by silica gel chromatography using PE/EA 4:1, white solid, mp $216-218^{\circ} \mathrm{C}, 81 \%$ yield, $37.7 \mathrm{mg}, 99 \%$ ee, $>20: 1 \mathrm{dr}$. The enantiomeric excess was determined by HPLC on Daicel Chiralpak IC-H with hexane/i-PrOH (90:10) as the eluent, flow $=1.0 \mathrm{~mL} / \mathrm{min}, \mathrm{UV}=254 \mathrm{~nm},[\alpha]_{D}^{25}$ $=-159\left(\mathrm{c}=1.0\right.$ in $\left.\mathrm{CH}_{2} \mathrm{Cl}_{2}\right) .{ }^{1} \mathbf{H}$ NMR $\left(500 \mathrm{MHz}, \mathrm{CDCl}_{3}\right) \delta 8.08-8.06(\mathrm{~m}, 2 \mathrm{H}), 7.91(\mathrm{~d}, J=6.6$ $\mathrm{Hz}, 1 \mathrm{H}), 7.53-7.49(\mathrm{~m}, 3 \mathrm{H}), 7.35-7.31(\mathrm{~m}, 3 \mathrm{H}), 7.28-7.27(\mathrm{~m}, 1 \mathrm{H}), 7.23(\mathrm{t}, J=7.7 \mathrm{~Hz}, 2 \mathrm{H}), 7.17-$ $7.12(\mathrm{~m}, 2 \mathrm{H}), 7.10(\mathrm{t}, J=8.5 \mathrm{~Hz}, 1 \mathrm{H}), 6.78(\mathrm{~d}, J=8.1 \mathrm{~Hz}, 1 \mathrm{H}), 6.71(\mathrm{~d}, J=7.4 \mathrm{~Hz}, 1 \mathrm{H}), 5.83(\mathrm{~s}$, 1H), 5.65 (s, 1H), 5.34 (s, 1H), 3.77 (s, 3H). ${ }^{13} \mathbf{C}$ NMR (126 MHz, $\left.\mathrm{CDCl}_{3}\right) \delta$ 199.8, 151.9, 144.6, 140.0, 137.7, 135.3, 134.7, 131.8, 130.5, 129.7, 129.3, 128.5, 128.4, 127.3, 127.0, 126.8, 120.7, 110.5, 106.3, 60.4, 55.5, 46.1. HRMS (ESI) $\mathrm{m} / \mathrm{z}$ : $[\mathrm{M}+\mathrm{Na}]+$ Calcd for $\mathrm{C}_{30} \mathrm{H}_{23} \mathrm{ClO}_{3} \mathrm{Na} 489.1228$; Found 489.1245 .

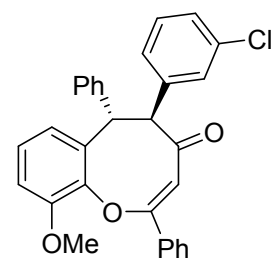

(5S,6S,Z)-5-(3-chlorophenyl)-10-methoxy-2,6-diphenyl-5,6-dihydro-4H-benzo[b]oxocin-4one (4aj). Purified by silica gel chromatography using PE/EA 4:1, white solid, mp 190-193 ${ }^{\circ} \mathrm{C}, 84 \%$ yield, $39.1 \mathrm{mg}, 97 \%$ ee, $>20: 1 \mathrm{dr}$. The enantiomeric excess was determined by HPLC on Daicel Chiralpak IB-H with hexane/i-PrOH (90:10) as the eluent, flow $=1.0 \mathrm{~mL} / \mathrm{min}, \mathrm{UV}=254 \mathrm{~nm},[\alpha]_{D}^{25}$ $=-237\left(\mathrm{c}=1.0\right.$ in $\left.\mathrm{CH}_{2} \mathrm{Cl}_{2}\right) .{ }^{1} \mathrm{H}$ NMR $\left(500 \mathrm{MHz}, \mathrm{CDCl}_{3}\right) \delta 8.11-8.11(\mathrm{~m}, 2 \mathrm{H}), 7.61(\mathrm{~s}, 1 \mathrm{H}), 7.55-$ 7.53 (m, 3H), 7.41 (d, $J=6.1 \mathrm{~Hz}, 1 \mathrm{H}), 7.33$ (d, $J=7.8 \mathrm{~Hz}, 2 \mathrm{H}), 7.27(\mathrm{t}, J=7.5 \mathrm{~Hz}, 2 \mathrm{H}), 7.22-7.18$ $(\mathrm{m}, 3 \mathrm{H}), 7.12(\mathrm{t}, J=8.0 \mathrm{~Hz}, 1 \mathrm{H}), 6.81(\mathrm{~d}, J=8.1 \mathrm{~Hz}, 1 \mathrm{H}), 6.71(\mathrm{~d}, J=7.4 \mathrm{~Hz}, 1 \mathrm{H}), 5.83(\mathrm{~s}, 1 \mathrm{H})$, 5.38 (s, 1H), 4.90 (s, 1H), 3.79 (s, 3H). ${ }^{13} \mathbf{C}$ NMR $\left(126 \mathrm{MHz}, \mathrm{CDCl}_{3}\right) \delta 200.2,151.7,144.4,139.7$, 137.9, 134.7, 134.3, 130.6, 129.6, 128.8, 128.5, 128.4, 127.6, 127.3, 127.0, 127.0, 126.9, 120.4, 110.4, 104.9, 66.3, 55.5, 44.6. HRMS (ESI) $\mathrm{m} / \mathrm{z}$ : $[\mathrm{M}+\mathrm{Na}]+$ Calcd for $\mathrm{C}_{30} \mathrm{H}_{23} \mathrm{ClO}_{3} \mathrm{Na}$ 489.1228; Found 489.1240 .

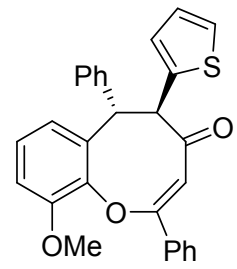

(5R,6S,Z)-10-methoxy-2,6-diphenyl-5-(thiophen-2-yl)-5,6-dihydro-4H-benzo[b]oxocin-4-one

(4ak). Purified by silica gel chromatography using PE/EA 4:1, white solid, mp $125-127^{\circ} \mathrm{C}, 74 \%$ yield, $32.4 \mathrm{mg}, 98 \%$ ee, $>20: 1 \mathrm{dr}$. The enantiomeric excess was determined by HPLC on Daicel Chiralpak IB-H with hexane/i-PrOH (95:5) as the eluent, flow $=1.0 \mathrm{~mL} / \mathrm{min}, \mathrm{UV}=254 \mathrm{~nm},[\alpha]_{D}^{25}$ $=-158\left(\mathrm{c}=1.0\right.$ in $\left.\mathrm{CH}_{2} \mathrm{Cl}_{2}\right) .{ }^{1} \mathbf{H}$ NMR $\left(500 \mathrm{MHz}, \mathrm{CDCl}_{3}\right) \delta 8.07-8.06(\mathrm{~m}, 2 \mathrm{H}), 7.53-7.48(\mathrm{~m}$, $3 \mathrm{H}), 7.32(\mathrm{~d}, J=7.2 \mathrm{~Hz}, 2 \mathrm{H}), 7.28-7.25(\mathrm{~m}, 2 \mathrm{H}), 7.21-7.18(\mathrm{~m}, 2 \mathrm{H}), 7.08(\mathrm{t}, J=8.0 \mathrm{~Hz}, 1 \mathrm{H}), 6.92-$ $6.85(\mathrm{~m}, 2 \mathrm{H}), 6.78(\mathrm{~d}, J=8.0 \mathrm{~Hz}, 1 \mathrm{H}), 6.65(\mathrm{~d}, J=7.4 \mathrm{~Hz}, 1 \mathrm{H}), 5.85(\mathrm{~s}, 1 \mathrm{H}), 5.14(\mathrm{~s}, 2 \mathrm{H}), 3.77(\mathrm{~s}$, 3H). ${ }^{13} \mathbf{C}$ NMR $\left(126 \mathrm{MHz}, \mathrm{CDCl}_{3}\right) \delta 199.9,151.8,140.7,137.7,134.8,132.1,130.5,128.5,128.5$, $128.3,127.4,126.9,126.7,126.4,125.3,120.6,110.5,62.2,55.5,26.9$. HRMS (ESI) m/z: [M + $\mathrm{Na}]+$ Calcd for $\mathrm{C}_{28} \mathrm{H}_{22} \mathrm{SO}_{3} \mathrm{Na}$ 461.1182; Found 461.1198. 


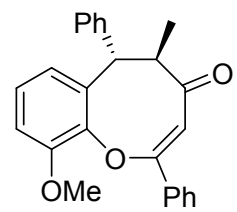

(5R,6S,Z)-10-methoxy-5-methyl-2,6-diphenyl-5,6-dihydro-4H-benzo[b]oxocin-4-one (4al). Purified by silica gel chromatography using PE/EA 4:1, white solid, mp 113-115 ${ }^{\circ} \mathrm{C}, 24 \%$ yield, 8.9 $\mathrm{mg}, 92 \% e e,>20: 1 \mathrm{dr}$. The enantiomeric excess was determined by HPLC on Daicel Chiralpak IB-H with hexane $/ \mathrm{i}-\mathrm{PrOH}(90: 10)$ as the eluent, flow $=1.0 \mathrm{~mL} / \mathrm{min}, \mathrm{UV}=254 \mathrm{~nm},[\alpha]_{D}^{25}=-178$ (c $=$ 1.0 in $\left.\mathrm{CH}_{2} \mathrm{Cl}_{2}\right) .{ }^{1} \mathbf{H}$ NMR $\left(600 \mathrm{MHz}, \mathrm{CDCl}_{3}\right) \delta 8.11-8.09(\mathrm{~m}, 4 \mathrm{H}), 7.52-7.51(\mathrm{~m}, 3 \mathrm{H}), 7.48(\mathrm{~d}, J=$ $7.8 \mathrm{~Hz}, 2 \mathrm{H}), 7.37$ (t, $J=7.6 \mathrm{~Hz}, 2 \mathrm{H}), 7.28(\mathrm{~s}, 1 \mathrm{H}), 7.01(\mathrm{t}, J=8.0 \mathrm{~Hz}, 1 \mathrm{H}), 6.71(\mathrm{~d}, J=7.2 \mathrm{~Hz}, 1 \mathrm{H})$, $6.66(\mathrm{~d}, J=7.9 \mathrm{~Hz}, 1 \mathrm{H}), 6.08(\mathrm{~s}, 1 \mathrm{H}), 4.30(\mathrm{~s}, 1 \mathrm{H}), 3.95(\mathrm{~s}, 1 \mathrm{H}), 3.66(\mathrm{~s}, 3 \mathrm{H}), 1.23(\mathrm{~d}, J=6.3 \mathrm{~Hz}$, 3H). ${ }^{13} \mathrm{C}$ NMR $\left(151 \mathrm{MHz}, \mathrm{CDCl}_{3}\right) \delta 205.7,152.3,145.6,141.9,138.3,134.7,130.5,128.8,128.5$, 128.3, 127.1, 127.0, 126.5, 121.3, 110.3, 55.4, 53.5, 51.1, 17.4. HRMS (ESI) m/z: [M + Na]+ Calcd for $\mathrm{C}_{25} \mathrm{H}_{22} \mathrm{O}_{3} \mathrm{Na} 393.1461$; Found 393.1469.

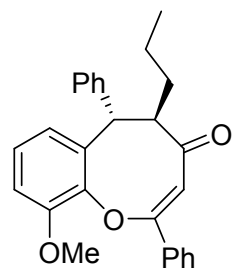

(5R,6S,Z)-10-methoxy-2,6-diphenyl-5-propyl-5,6-dihydro-4H-benzo[b]oxocin-4-one (4am). Purified by silica gel chromatography using PE/EA 4:1, white solid, mp 136-138 ${ }^{\circ} \mathrm{C}, 17 \%$ yield, 6.8 mg, 93\% ee, $>20: 1 \mathrm{dr}$. The enantiomeric excess was determined by HPLC on Daicel Chiralpak IB-H with hexane $/ \mathrm{i}-\mathrm{PrOH}(90: 10)$ as the eluent, flow $=1.0 \mathrm{~mL} / \mathrm{min}, \mathrm{UV}=254 \mathrm{~nm},[\alpha]_{D}^{25}=-159(\mathrm{c}=$ 1.0 in $\left.\mathrm{CH}_{2} \mathrm{Cl}_{2}\right)$. ${ }^{1} \mathrm{H}$ NMR $\left(500 \mathrm{MHz}, \mathrm{CDCl}_{3}\right) \delta 8.08(\mathrm{dd}, J=6.6,2.9 \mathrm{~Hz}, 1 \mathrm{H}), 7.51-7.46(\mathrm{~m}, 5 \mathrm{H})$, $7.36(\mathrm{t}, J=7.6 \mathrm{~Hz}, 2 \mathrm{H}), 7.26-7.25(\mathrm{~m}, 1 \mathrm{H}), 6.99(\mathrm{t}, J=8.0 \mathrm{~Hz}, 1 \mathrm{H}), 6.69(\mathrm{~d}, J=7.8 \mathrm{~Hz}, 1 \mathrm{H}), 6.63$ $(\mathrm{d}, J=7.7 \mathrm{~Hz}, 1 \mathrm{H}), 6.04(\mathrm{~s}, 1 \mathrm{H}), 4.30(\mathrm{~s}, 1 \mathrm{H}), 3.82(\mathrm{~s}, 1 \mathrm{H}), 3.65(\mathrm{~s}, 3 \mathrm{H}), 1.61-1.17(\mathrm{~m}, 4 \mathrm{H}), 0.82(\mathrm{t}$, $J=7.3 \mathrm{~Hz}, 3 \mathrm{H}) .{ }^{13} \mathrm{C} \mathrm{NMR}\left(126 \mathrm{MHz}, \mathrm{CDCl}_{3}\right) \delta 199.4,152.2,145.2,138.2,134.6,132.1,130.4$, $128.8,128.5,128.2,127.1,126.9,126.4,121.0,110.2,61.6,55.3,51.8,34.3,20.8,14.0$. HRMS (ESI) m/z: $[\mathrm{M}+\mathrm{Na}]+$ Calcd for $\mathrm{C}_{27} \mathrm{H}_{26} \mathrm{O}_{3} \mathrm{Na}$ 421.1774; Found 421.1782.<smiles>COc1cccc2c1O/C(c1ccccc1)=C\C(=O)C(OCc1ccccc1)C2c1ccccc1</smiles>

(5R,6S,Z)-5-(benzyloxy)-10-methoxy-2,6-diphenyl-5,6-dihydro-4H-benzo[b]oxocin-4-one (4an). Purified by silica gel chromatography using PE/EA 4:1, white solid, mp 89-91 ${ }^{\circ} \mathrm{C}, 78 \%$ yield, 36.2 mg, 95\% ee, $>20: 1 \mathrm{dr}$. The enantiomeric excess was determined by HPLC on Daicel Chiralpak IB-H with hexane/i-PrOH (95:5) as the eluent, flow $=1.0 \mathrm{~mL} / \mathrm{min}, \mathrm{UV}=254 \mathrm{~nm},[\alpha]_{D}^{25}=-201 \quad(\mathrm{c}=$ 1.0 in $\left.\mathrm{CH}_{2} \mathrm{Cl}_{2}\right) .{ }^{1} \mathbf{H}$ NMR $\left(600 \mathrm{MHz}, \mathrm{CDCl}_{3}\right) \delta 8.06-8.05(\mathrm{~m}, 2 \mathrm{H}), 7.52-7.48(\mathrm{~m}, 3 \mathrm{H}), 7.42(\mathrm{~s}, 2 \mathrm{H})$ $7.34(\mathrm{t}, J=7.5 \mathrm{~Hz}, 2 \mathrm{H}), 7.30-7.28(\mathrm{~m}, 2 \mathrm{H}), 7.25(\mathrm{~s}, 3 \mathrm{H}), 7.12(\mathrm{~s}, 2 \mathrm{H}), 7.04(\mathrm{t}, J=8.0 \mathrm{~Hz}, 1 \mathrm{H}), 6.74$ $(\mathrm{d}, J=8.0 \mathrm{~Hz}, 1 \mathrm{H}), 6.67-6.66(\mathrm{~m}, 1 \mathrm{H}), 6.10(\mathrm{~s}, 1 \mathrm{H}), 5.07(\mathrm{~s}, 1 \mathrm{H}), 4.54(\mathrm{~d}, J=11.8 \mathrm{~Hz}, 1 \mathrm{H}), 4.36$ $(\mathrm{d}, J=11.0 \mathrm{~Hz}, 1 \mathrm{H}), 3.63(\mathrm{~s}, 3 \mathrm{H}) .{ }^{13} \mathbf{C} \mathbf{N M R}\left(126 \mathrm{MHz}, \mathrm{CDCl}_{3}\right) \delta 201.1,152.1,137.4,135.0,130.7$, 128.6, 128.5, 128.2, 128.2, 128.0, 127.6, 127.2, 126.9, 126.7, 110.8, 72.2, 55.3, 26.9. HRMS (ESI) $\mathrm{m} / \mathrm{z}:[\mathrm{M}+\mathrm{Na}]+$ Calcd for $\mathrm{C}_{31} \mathrm{H}_{26} \mathrm{O}_{4} \mathrm{Na} 485.1723$; Found 485.1737. 
<smiles>COc1ccc2c(c1)O/C(c1ccccc1)=C\C(=O)C(Oc1ccccc1)C2c1ccccc1</smiles>

(5R,6S,Z)-5-(benzyloxy)-9-methoxy-2,6-diphenyl-5,6-dihydro-4H-benzo[b]oxocin-4-one (4bn). Purified by silica gel chromatography using PE/EA 4:1, white solid, mp $158-160{ }^{\circ} \mathrm{C}, 75 \%$ yield, $34.7 \mathrm{mg}, 94 \%$ ee, $>20: 1 \mathrm{dr}$. The enantiomeric excess was determined by HPLC on Daicel Chiralpak AD-H with hexane/i-PrOH (90:10) as the eluent, flow $=1.0 \mathrm{~mL} / \mathrm{min}, \mathrm{UV}=254 \mathrm{~nm},[\alpha]_{D}^{25}=-158$ (c $=1.0$ in $\mathrm{CH}_{2} \mathrm{Cl}_{2}$ ). ${ }^{1} \mathbf{H}$ NMR (400 MHz, Chloroform- $d$ ) $\delta$ 8.01-7.99 (m, $\left.2 \mathrm{H}\right), 7.58-7.55(\mathrm{~m}, 3 \mathrm{H})$, $7.48(\mathrm{~d}, J=7.3 \mathrm{~Hz}, 2 \mathrm{H}), 7.38-7.31(\mathrm{~m}, 3 \mathrm{H}), 7.22-7.19(\mathrm{~m}, J=5.5 \mathrm{~Hz}, 3 \mathrm{H}), 7.08(\mathrm{~d}, J=9.2 \mathrm{~Hz}, 1 \mathrm{H})$, $7.03(\mathrm{~d}, J=5.4 \mathrm{~Hz}, 2 \mathrm{H}), 6.68-6.66(\mathrm{~m}, 2 \mathrm{H}), 6.37(\mathrm{~s}, 1 \mathrm{H}), 5.44(\mathrm{~d}, J=7.32 \mathrm{~Hz}, 1 \mathrm{H}), 4.53(\mathrm{~d}, J=$ $12.0 \mathrm{~Hz}, 1 \mathrm{H}), 4.33(\mathrm{~d}, J=8.8 \mathrm{~Hz}, 1 \mathrm{H}), 4.28(\mathrm{~d}, J=12.0 \mathrm{~Hz}, 1 \mathrm{H}), 3.67(\mathrm{~s}, 3 \mathrm{H}) .{ }^{13} \mathbf{C}$ NMR $(100 \mathrm{MHz}$, $\left.\mathrm{CDCl}_{3}\right) \delta 202.1,159.3,158.2,143.2,137.5,133.6,132.9,131.6,129.1,128.6,128.5,128.2,127.8$, 127.5, 126.9, 126.8, 125.0, 112.3, 107.3, 86.5, 72.2, 55.5. HRMS (ESI) m/z: [M + Na]+ Calcd for $\mathrm{C}_{31} \mathrm{H}_{26} \mathrm{O}_{4} \mathrm{Na}$ 485.1723; Found 485.1731.<smiles>O=C1/C=C(\c2ccccc2)C(O)C(c2ccccc2)c2cccc(F)c2O1</smiles>

(5R,6S,Z)-5-(benzyloxy)-10-fluoro-2,6-diphenyl-5,6-dihydro-4H-benzo[b]oxocin-4-one

(4gn). Purified by silica gel chromatography using PE/EA 4:1, white solid, mp $113-115{ }^{\circ} \mathrm{C}, 69 \%$ yield, $31.0 \mathrm{mg}, 94 \%$ ee, $>20: 1 \mathrm{dr}$. The enantiomeric excess was determined by HPLC on Daicel Chiralpak IB-H with hexane/i-PrOH (95:5) as the eluent, flow $=1.0 \mathrm{~mL} / \mathrm{min}, \mathrm{UV}=254 \mathrm{~nm},[\alpha]_{D}^{25}=-158$ $\left(\mathrm{c}=1.0\right.$ in $\left.\mathrm{CH}_{2} \mathrm{Cl}_{2}\right) .{ }^{1} \mathbf{H}$ NMR $\left(600 \mathrm{MHz}, \mathrm{CDCl}_{3}\right) \delta 8.00(\mathrm{~d}, J=8.0 \mathrm{~Hz}, 2 \mathrm{H}), 7.55-7.51(\mathrm{~m}, 3 \mathrm{H})$, 7.43 (d, $J=7.4 \mathrm{~Hz}, 2 \mathrm{H}), 7.36$ (t, $J=7.4 \mathrm{~Hz}, 2 \mathrm{H}), 7.30$ (t, $J=7.3 \mathrm{~Hz}, 1 \mathrm{H}), 7.25-7.23$ (m 3H), 7.07 $(\mathrm{d}, J=7.4 \mathrm{~Hz}, 2 \mathrm{H}), 7.06-7.03(\mathrm{~m}, 1 \mathrm{H}), 6.96(\mathrm{ddd}, J=9.9,8.2,1.5 \mathrm{~Hz}, 1 \mathrm{H}), 6.91(\mathrm{~d}, J=7.8 \mathrm{~Hz}, 1 \mathrm{H})$, $6.23(\mathrm{~s}, 1 \mathrm{H}), 5.32(\mathrm{~s}, 1 \mathrm{H}), 5.22(\mathrm{~s}, 1 \mathrm{H}), 4.52(\mathrm{~d}, J=11.9 \mathrm{~Hz}, 2 \mathrm{H}), 4.33(\mathrm{~d}, J=11.9 \mathrm{~Hz}, 1 \mathrm{H}) .{ }^{13} \mathrm{C}$ NMR $\left(151 \mathrm{MHz}, \mathrm{CDCl}_{3}\right) \delta 201.1,155.5\left(\mathrm{~d},{ }^{1} J_{\mathrm{C}-\mathrm{F}}=249.6 \mathrm{~Hz}\right), 145.0,141.6,137.3,136.3,132.8$, $131.3,128.6,128.5,128.2,128.0,127.7,127.1,127.0\left(\mathrm{~d},{ }^{3} J_{\mathrm{C}-\mathrm{F}}=7.8 \mathrm{~Hz}\right), 126.8\left(\mathrm{~d},{ }^{4} J_{\mathrm{C}-\mathrm{F}}=2.5 \mathrm{~Hz}\right)$, 126.4, $115.5\left(\mathrm{~d},{ }^{2} J_{\mathrm{C}-\mathrm{F}}=18.9 \mathrm{~Hz}\right), 86.8,72.3,53.4,50.9$. HRMS (ESI) m/z: $[\mathrm{M}+\mathrm{Na}]+$ Calcd for $\mathrm{C}_{30} \mathrm{H}_{23} \mathrm{FO}_{3} \mathrm{Na}$ 473.1523; Found 473.1527.

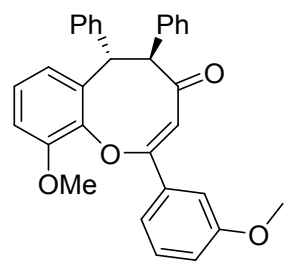

(5S,6S,Z)-10-methoxy-2-(3-methoxyphenyl)-5,6-diphenyl-5,6-dihydro-4H-benzo[b]oxocin-4one (4ao). Purified by silica gel chromatography using PE/EA 4:1, white solid, mp 172-174 ${ }^{\circ} \mathrm{C}$, $79 \%$ yield, $36.4 \mathrm{mg}$, 98\% ee, $>20: 1 \mathrm{dr}$. The enantiomeric excess was determined by HPLC on Daicel Chiralpak IB-H with hexane/i-PrOH (95:5) as the eluent, flow $=1.0 \mathrm{~mL} / \mathrm{min}, \mathrm{UV}=254 \mathrm{~nm},[\alpha]_{D}^{25}$ $=-296\left(\mathrm{c}=1.0\right.$ in $\left.\mathrm{CH}_{2} \mathrm{Cl}_{2}\right) .{ }^{1} \mathbf{H}$ NMR $\left(500 \mathrm{MHz}, \mathrm{CDCl}_{3}\right) \delta 7.70(\mathrm{t}, J=1.9 \mathrm{~Hz}, 1 \mathrm{H}), 7.54-7.51$ (m, 3H), 7.39 (t, $J=8.0 \mathrm{~Hz}, 1 \mathrm{H}), 7.30-7.27(\mathrm{~m}, 4 \mathrm{H}), 7.25-7.20(\mathrm{~m}, 3 \mathrm{H}), 7.16(\mathrm{t}, J=7.3 \mathrm{~Hz}, 1 \mathrm{H})$, $7.11(\mathrm{t}, J=8.0 \mathrm{~Hz}, 1 \mathrm{H}), 7.06(\mathrm{ddd}, J=8.2,2.6,0.8 \mathrm{~Hz}, 1 \mathrm{H}), 6.80(\mathrm{dd}, J=8.2,1.2 \mathrm{~Hz}, 1 \mathrm{H}), 6.65(\mathrm{~d}$, $J=7.8 \mathrm{~Hz}, 1 \mathrm{H}), 5.68(\mathrm{~s}, 1 \mathrm{H}), 5.51(\mathrm{~s}, 1 \mathrm{H}), 4.77(\mathrm{~s}, 1 \mathrm{H}), 3.92(\mathrm{~s}, 3 \mathrm{H}), 3.83(\mathrm{~s}, 3 \mathrm{H}) .{ }^{13} \mathbf{C}$ NMR $(126$ 
$\left.\mathrm{MHz}, \mathrm{CDCl}_{3}\right) \delta 201.9,157.5,151.8,143.9,140.2,138.6,137.7,131.0,130.5,129.1,128.7,128.3$, 128.2, 127.2, 126.8, 126.6, 123.8, 120.2, 111.1, 110.2, 67.0, 55.5, 55.3, 45.8. HRMS (ESI) m/z: [M $+\mathrm{Na}]+$ Calcd for $\mathrm{C}_{31} \mathrm{H}_{26} \mathrm{O}_{4} \mathrm{Na} 485.1723$; Found 485.1728.

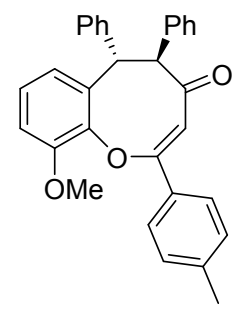

(5S,6S,Z)-10-methoxy-5,6-diphenyl-2-(p-tolyl)-5,6-dihydro-4H-benzo[b]oxocin-4-one (4ap). Purified by silica gel chromatography using PE/EA 4:1, white solid, mp 226-228 ${ }^{\circ} \mathrm{C}$, 83\% yield, $37.0 \mathrm{mg}, 97 \% e e,>20: 1 \mathrm{dr}$. The enantiomeric excess was determined by HPLC on Daicel Chiralpak IB-H with hexane/i-PrOH (90:10) as the eluent, flow $=1.0 \mathrm{~mL} / \mathrm{min}, \mathrm{UV}=254 \mathrm{~nm},[\alpha]_{D}^{25}=-255$ (c = 1.0 in $\left.\mathrm{CH}_{2} \mathrm{Cl}_{2}\right) .{ }^{1} \mathbf{H}$ NMR $\left(500 \mathrm{MHz}, \mathrm{CDCl}_{3}\right) \delta 7.94(\mathrm{~d}, J=8.1 \mathrm{~Hz}, 2 \mathrm{H}), 7.51(\mathrm{~d}, J=7.4 \mathrm{~Hz}$, 2H), 7.32-7.26 (m, 6H), 7.24-7.20 (m, 3H), $7.15(\mathrm{t}, J=7.3 \mathrm{~Hz}, 1 \mathrm{H}), 7.10(\mathrm{t}, J=8.0 \mathrm{~Hz}, 1 \mathrm{H}), 6.79$ (dd, $J=8.2,1.2 \mathrm{~Hz}, 1 \mathrm{H}), 6.65(\mathrm{~d}, J=7.7 \mathrm{~Hz}, 1 \mathrm{H}), 5.69(\mathrm{~s}, 1 \mathrm{H}), 5.47$ (s, 1H), $4.80(\mathrm{~s}, 1 \mathrm{H}), 3.81(\mathrm{~s}$, 3H), $2.47(\mathrm{~s}, 3 \mathrm{H}) .{ }^{13} \mathbf{C}$ NMR $\left(126 \mathrm{MHz}, \mathrm{CDCl}_{3}\right) \delta 201.0,151.7,144.3,140.8,139.9,138.6,137.7$, 132.2, 129.1, 128.8, 128.5, 128.4, 127.4, 127.4, 127.0, 126.7, 120.3, 110.4, 67.2, 55.6, 41.5, 21.5. HRMS (ESI) m/z: [M + Na]+ Calcd for $\mathrm{C}_{31} \mathrm{H}_{26} \mathrm{O}_{3} \mathrm{Na}$ 469.1774; Found 469.1782.<smiles>COc1cccc2c1OC(c1ccc(F)cc1)C(=O)C(c1ccccc1)c1ccccc1-2</smiles>

\section{(5S,6S,Z)-2-(4-fluorophenyl)-10-methoxy-5,6-diphenyl-5,6-dihydro-4H-benzo[b]oxocin-4-one}

(4aq). Purified by silica gel chromatography using PE/EA 4:1, white solid, mp 251-253 ${ }^{\circ} \mathrm{C}, 75 \%$ yield, $33.7 \mathrm{mg}, 90 \% e e,>20: 1 \mathrm{dr}$. The enantiomeric excess was determined by HPLC on Daicel Chiralpak IB-H with hexane/i-PrOH (90:10) as the eluent, flow $=1.0 \mathrm{~mL} / \mathrm{min}, \mathrm{UV}=254 \mathrm{~nm},[\alpha]_{D}^{25}$ $=-256\left(\mathrm{c}=1.0\right.$ in $\left.\mathrm{CH}_{2} \mathrm{Cl}_{2}\right) .{ }^{1} \mathbf{H}$ NMR $\left(500 \mathrm{MHz}, \mathrm{CDCl}_{3}\right) \delta 8.02(\mathrm{dd}, J=8.6,5.4 \mathrm{~Hz}, 2 \mathrm{H}), 7.50$ $(\mathrm{d}, J=7.3 \mathrm{~Hz}, 2 \mathrm{H}), 7,30-7.27(\mathrm{~m}, 4 \mathrm{H}), 7.24-7.15(\mathrm{~m}, 6 \mathrm{H}), 7.10(\mathrm{t}, J=8.0 \mathrm{~Hz}, 1 \mathrm{H}), 6.79(\mathrm{dd}, J=$ $8.2,1.2 \mathrm{~Hz}, 1 \mathrm{H}), 6.65(\mathrm{~d}, J=7.7 \mathrm{~Hz}, 1 \mathrm{H}), 5.63(\mathrm{~s}, 1 \mathrm{H}), 5.46(\mathrm{~s}, 1 \mathrm{H}), 4.80(\mathrm{~s}, 1 \mathrm{H}), 3.82(\mathrm{~s}, 3 \mathrm{H}) .{ }^{13} \mathrm{C}$ NMR $\left(126 \mathrm{MHz}, \mathrm{CDCl}_{3}\right) \delta 200.7,164.2\left(\mathrm{~d},{ }^{4} J_{\mathrm{C}-\mathrm{F}}=249.4 \mathrm{~Hz}\right), 151.6,144.0,139.8,138.5,137.5$, $131.2,129.5\left(\mathrm{~d},{ }^{3} J_{\mathrm{C}-\mathrm{F}}=8.5 \mathrm{~Hz}\right), 128.7,128.6,128.4,127.5,127.1,126.7,120.3,115.4\left(\mathrm{~d},{ }^{2} J_{\mathrm{C}-\mathrm{F}}=21.7\right.$ $\mathrm{Hz}$ ), 110.3, 105.4, 67.0, 55.6, 43.9. HRMS (ESI) m/z: $[\mathrm{M}+\mathrm{Na}]+$ Calcd for $\mathrm{C}_{30} \mathrm{H}_{23} \mathrm{FO}_{3} \mathrm{Na}$ 473.1523; Found 473.1525 .

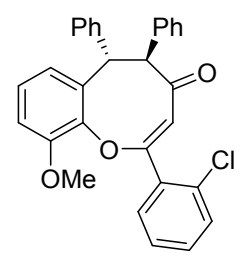

(5S,6S,Z)-2-(2-chlorophenyl)-10-methoxy-5,6-diphenyl-5,6-dihydro-4H-benzo[b]oxocin-4-one

(4ar). Purified by silica gel chromatography using PE/EA 4:1, white solid, mp 104-106 ${ }^{\circ} \mathrm{C}, 84 \%$ yield, $39.1 \mathrm{mg}, 97 \%$ ee, $>20: 1 \mathrm{dr}$. The enantiomeric excess was determined by HPLC on Daicel 
Chiralpak ID-H with hexane/i-PrOH (90:10) as the eluent, flow $=1.0 \mathrm{~mL} / \mathrm{min}, \mathrm{UV}=254 \mathrm{~nm},[\alpha]_{D}^{25}$ $=-209\left(\mathrm{c}=1.0\right.$ in $\left.\mathrm{CH}_{2} \mathrm{Cl}_{2}\right) .{ }^{1} \mathbf{H}$ NMR $\left(500 \mathrm{MHz}, \mathrm{CDCl}_{3}\right) \delta 8.08(\mathrm{~d}, J=7.0 \mathrm{~Hz}, 1 \mathrm{H}), 7.58(\mathrm{~d}, J=$ $7.6 \mathrm{~Hz}, 2 \mathrm{H}), 7.50$ (dd, $J=7.6,1.6 \mathrm{~Hz}, 1 \mathrm{H}), 7.40$ (pd, $J=7.4,1.8 \mathrm{~Hz}, 2 \mathrm{H}), 7.32-7.31(\mathrm{~m}, 2 \mathrm{H}), 7.29-$ $7.24(\mathrm{~m}, 4 \mathrm{H}), 7.22-7.12(\mathrm{~m}, 2 \mathrm{H}), 7.12(\mathrm{t}, J=8.0 \mathrm{~Hz}, 1 \mathrm{H}), 6.79(\mathrm{dd}, J=8.2,1.1 \mathrm{~Hz}, 1 \mathrm{H}), 6.64(\mathrm{~d}, J$ $=7.8 \mathrm{~Hz}, 1 \mathrm{H}), 5.70(\mathrm{~s}, 1 \mathrm{H}), 5.48(\mathrm{~s}, 1 \mathrm{H}), 4.75(\mathrm{~s}, 1 \mathrm{H}), 3.84(\mathrm{~s}, 3 \mathrm{H}) .{ }^{13} \mathbf{C ~ N M R}\left(126 \mathrm{MHz}, \mathrm{CDCl}_{3}\right) \delta$ 200.5, 157.4, 151.2, 142.8, 139.3, 138.6, 136.5, 134.9, 132.4, 131.0, 130.4, 130.0, 128.8, 128.4, 128.4, 127.4, 127.2, 126.7, 126.6, 119.9, 110.1, 109.5, 67.1, 55.5, 43.2. HRMS (ESI) m/z: [M + $\mathrm{Na}+$ Calcd for $\mathrm{C}_{30} \mathrm{H}_{23} \mathrm{ClO}_{3} \mathrm{Na}$ 489.1228; Found 489.1242.

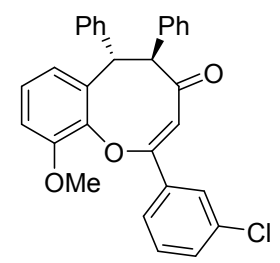

\section{(5S,6S,Z)-2-(3-chlorophenyl)-10-methoxy-5,6-diphenyl-5,6-dihydro-4H-benzo[b]oxocin-4-one}

(4as). Purified by silica gel chromatography using PE/EA 4:1, white solid, mp 205-207 ${ }^{\circ} \mathrm{C}, 70 \%$ yield, $32.6 \mathrm{mg}, 96 \%$ ee, $>20: 1 \mathrm{dr}$. The enantiomeric excess was determined by HPLC on Daicel Chiralpak IB-H with hexane/i-PrOH (95:5) as the eluent, flow $=1.0 \mathrm{~mL} / \mathrm{min}, \mathrm{UV}=254 \mathrm{~nm},[\alpha]_{D}^{25}$ $=-240\left(\mathrm{c}=1.0\right.$ in $\left.\mathrm{CH}_{2} \mathrm{Cl}_{2}\right) .{ }^{1} \mathbf{H} \mathbf{~ N M R}\left(500 \mathrm{MHz}, \mathrm{CDCl}_{3}\right) \delta 8.12(\mathrm{~s}, 1 \mathrm{H}), 7.82(\mathrm{~d}, J=7.8 \mathrm{~Hz}, 1 \mathrm{H})$, 7.50-7.47 (m, 3H), $7.40(\mathrm{t}, J=7.9 \mathrm{~Hz}, 1 \mathrm{H}), 7.30-7.27(\mathrm{~m}, 4 \mathrm{H}), 7.25-7.22(\mathrm{~m}, 3 \mathrm{H}), 7.16(\mathrm{t}, J=7.2$ $\mathrm{Hz}, 1 \mathrm{H}), 7.11$ (t, $J=8.0 \mathrm{~Hz}, 1 \mathrm{H}), 6.79(\mathrm{dd}, J=8.2 \mathrm{~Hz}, 0.8 \mathrm{~Hz}, 1 \mathrm{H}), 6.65(\mathrm{~d}, J=7.7 \mathrm{~Hz}, 1 \mathrm{H}), 5.70$ $(\mathrm{s}, 1 \mathrm{H}), 5.44(\mathrm{~s}, 1 \mathrm{H}), 4.81(\mathrm{~s}, 1 \mathrm{H}), 3.82(\mathrm{~s}, 3 \mathrm{H}) .{ }^{13} \mathbf{C} \mathbf{~ N M R}\left(126 \mathrm{MHz}, \mathrm{CDCl}_{3}\right) \delta 200.6,151.6,143.8$, 139.8, 138.4, 137.3, 136.8, 134.5, 130.2, 129.6, 128.7, 128.6, 128.4, 127.6, 127.5, 127.2, 126.7, $125.3,120.3,110.3,106.4,67.0,55.5,44.0$. HRMS (ESI) $\mathrm{m} / \mathrm{z}$ : $[\mathrm{M}+\mathrm{Na}]+$ Calcd for $\mathrm{C}_{30} \mathrm{H}_{23} \mathrm{ClO}_{3} \mathrm{Na}$ 489.1228; Found 489.1240.

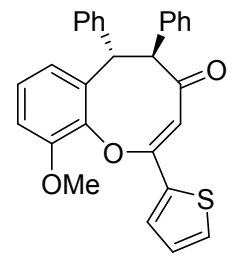

(5S,6S,Z)-10-methoxy-5,6-diphenyl-2-(thiophen-2-yl)-5,6-dihydro-4H-benzo[b]oxocin-4-one

(4at). Purified by silica gel chromatography using PE/EA 4:1, white solid, mp 120-122 ${ }^{\circ} \mathrm{C}, 78 \%$ yield, $34.1 \mathrm{mg}, 96 \%$ ee, $>20: 1 \mathrm{dr}$. The enantiomeric excess was determined by HPLC on Daicel Chiralpak IC-H with hexane/i-PrOH (90:10) as the eluent, flow $=1.0 \mathrm{~mL} / \mathrm{min}, \mathrm{UV}=254 \mathrm{~nm},[\alpha]_{D}^{25}$ $=-71\left(\mathrm{c}=1.0\right.$ in $\left.\mathrm{CH}_{2} \mathrm{Cl}_{2}\right) .{ }^{1} \mathbf{H}$ NMR $\left(500 \mathrm{MHz}, \mathrm{CDCl}_{3}\right) \delta 7.74(\mathrm{~s}, 1 \mathrm{H}), 7.54(\mathrm{~d}, J=4.9 \mathrm{~Hz}, 1 \mathrm{H})$, $7.48(\mathrm{~d}, J=7.4 \mathrm{~Hz}, 2 \mathrm{H}), 7.26(\mathrm{t}, J=7.8 \mathrm{~Hz}, 4 \mathrm{H}), 7.22-7.19(\mathrm{~m}, 3 \mathrm{H}), 7.16(\mathrm{dd}, J=5.0,3.8 \mathrm{~Hz}, 1 \mathrm{H})$, $7.13(\mathrm{t}, J=7.2 \mathrm{~Hz}, 1 \mathrm{H}), 7.09$ (t, $J=8.0 \mathrm{~Hz}, 1 \mathrm{H}), 6.78(\mathrm{dd}, J=8.2,1.3 \mathrm{~Hz}, 1 \mathrm{H}), 6.67(\mathrm{~d}, J=7.0 \mathrm{~Hz}$, $1 \mathrm{H}), 5.80(\mathrm{~s}, 1 \mathrm{H}), 5.27(\mathrm{~s}, 1 \mathrm{H}), 4.90(\mathrm{~s}, 1 \mathrm{H}), 3.81(\mathrm{~s}, 3 \mathrm{H}) .{ }^{13} \mathrm{C} \mathrm{NMR}\left(126 \mathrm{MHz}, \mathrm{CDCl}_{3}\right) \delta 200.41$, 151.97, 138.64, 138.13, 137.74, 129.36, 128.93, 128.57, 128.42, 128.39, 127.75, 127.33, 127.04, 126.68, 120.83, 110.43, 66.22, 55.47, 53.40. HRMS (ESI) m/z: [M + Na]+ Calcd for $\mathrm{C}_{28} \mathrm{H}_{22} \mathrm{O}_{3} \mathrm{SNa}$ 461.1182; Found 461.1195. 


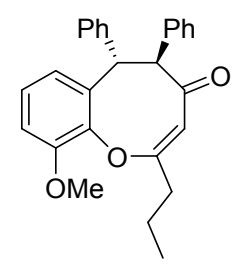

(5S,6S,Z)-10-methoxy-5,6-diphenyl-2-propyl-5,6-dihydro-4H-benzo[b]oxocin-4-one

(4au).

Purified by silica gel chromatography using PE/EA 4:1, white solid, mp 174-176 ${ }^{\circ} \mathrm{C}, 70 \%$ yield, $27.8 \mathrm{mg}, 97 \%$ ee, $>20: 1 \mathrm{dr}$. The enantiomeric excess was determined by HPLC on Daicel Chiralpak IC-H with hexane/i-PrOH $(90: 10)$ as the eluent, flow $=1.0 \mathrm{~mL} / \mathrm{min}, \mathrm{UV}=254 \mathrm{~nm},[\alpha]_{D}^{25}$ $=-91.000$ (c=1.0 in $\left.\mathrm{CH}_{2} \mathrm{Cl}_{2}\right) .{ }^{1} \mathbf{H} \mathbf{N M R}\left(500 \mathrm{MHz}, \mathrm{CDCl}_{3}\right) \delta 7.44(\mathrm{~d}, J=7.3 \mathrm{~Hz}, 2 \mathrm{H}), 7.27-7.19$ $(\mathrm{m}, 7 \mathrm{H}), 7.13(\mathrm{t}, J=7.0 \mathrm{~Hz}, 1 \mathrm{H}), 7.04(\mathrm{t}, J=8.0 \mathrm{~Hz}, 1 \mathrm{H}), 6.75(\mathrm{dd}, J=8.2,1.3 \mathrm{~Hz}, 1 \mathrm{H}), 6.59(\mathrm{~d}, J$ $=7.7 \mathrm{~Hz}, 1 \mathrm{H}), 5.30(\mathrm{~s}, 1 \mathrm{H}), 5.06(\mathrm{~s}, 1 \mathrm{H}), 4.71(\mathrm{~s}, 1 \mathrm{H}), 3.85(\mathrm{~s}, 3 \mathrm{H}), 2.63-2.49(\mathrm{~m}, 3 \mathrm{H}), 1.78-1.90$ $(\mathrm{m}, 2 \mathrm{H}), 1.10(\mathrm{t}, J=7.4 \mathrm{~Hz}, 3 \mathrm{H}) .{ }^{13} \mathbf{C} \mathbf{N M R}\left(126 \mathrm{MHz}, \mathrm{CDCl}_{3}\right) \delta 201.7,151.5,143.8,140.1,138.1$, $137.5,128.7,128.7,128.4,128.4,127.3,126.7,126.6,120.5,110.1,106.7,66.7,55.7,45.1,37.7$, 20.3, 13.7. HRMS (ESI) m/z: [M + Na]+ Calcd for $\mathrm{C}_{27} \mathrm{H}_{26} \mathrm{O}_{3} \mathrm{Na}$ 421.1774; Found 421.1789.<smiles>COc1cccc2c1O/C(C(C)(C)C)=C\C(=O)C(c1ccccc1)C(c1ccccc1)c1ccccc1-2</smiles>

(5S,6S,Z)-2-(tert-butyl)-10-methoxy-5,6-diphenyl-5,6-dihydro-4H-benzo[b]oxocin-4-one

(4av). Purified by silica gel chromatography using PE/EA 4:1, white solid, mp $134-136{ }^{\circ} \mathrm{C}, 62 \%$ yield, $25.5 \mathrm{mg}$, 93\% ee, $>20: 1 \mathrm{dr}$. The enantiomeric excess was determined by HPLC on Daicel Chiralpak IB-H with hexane/i-PrOH (95:5) as the eluent, flow $=1.0 \mathrm{~mL} / \mathrm{min}, \mathrm{UV}=254 \mathrm{~nm},[\alpha]_{D}^{25}$ $=-121.000$ (c = 1.0 in $\left.\mathrm{CH}_{2} \mathrm{Cl}_{2}\right) .{ }^{1} \mathbf{H} \mathbf{N M R}\left(500 \mathrm{MHz}, \mathrm{CDCl}_{3}\right) \delta 7.47(\mathrm{~d}, J=7.4 \mathrm{~Hz}, 2 \mathrm{H}), 7.27$ $(\mathrm{dt}, J=9.7,7.6 \mathrm{~Hz}, 10 \mathrm{H}), 7.19(\mathrm{dt}, J=13.9,7.2 \mathrm{~Hz}, 2 \mathrm{H}), 7.08(\mathrm{t}, J=8.0 \mathrm{~Hz}, 1 \mathrm{H}), 6.74(\mathrm{dd}, J=8.2$, $1.0 \mathrm{~Hz}, 1 \mathrm{H}), 6.53(\mathrm{dd}, J=7.9,1.1 \mathrm{~Hz}, 1 \mathrm{H}), 5.55(\mathrm{~d}, J=12.7 \mathrm{~Hz}, 1 \mathrm{H}), 4.92(\mathrm{~s}, 2 \mathrm{H}), 4.48(\mathrm{~d}, J=12.7$ $\mathrm{Hz}, 7 \mathrm{H}), 3.81(\mathrm{~s}, 3 \mathrm{H}), 1.43(\mathrm{~s}, 8 \mathrm{H}) .{ }^{13} \mathbf{C} \mathbf{N M R}\left(126 \mathrm{MHz}, \mathrm{CDCl}_{3}\right) \delta 202.2,169.8,151.1,142.3$, 139.0, 137.3, 129.1, 128.6, 128.4, 128.3, 127.4, 127.0, 126.7, 119.1, 110.0, 99.2, 68.3, 55.4, 41.7, 37.7, 28.6. HRMS (ESI) m/z: [M+ Na]+ Calcd for $\mathrm{C}_{28} \mathrm{H}_{28} \mathrm{O}_{3} \mathrm{Na}$ 435.1931; Found 435.1945.<smiles>CC(C)(C)C1=CC(=O)C(c2ccccc2)C(c2ccccc2)c2cccc(F)c2O1</smiles>

(5S,6S,Z)-2-(tert-butyl)-10-fluoro-5,6-diphenyl-5,6-dihydro-4H-benzo[b]oxocin-4-one (4gv). Purified by silica gel chromatography using PE/EA 4:1, white solid, mp 89-92 ${ }^{\circ} \mathrm{C}$, 65\% yield, 26.0 $\mathrm{mg}, 85 \%$ ee, $>20: 1 \mathrm{dr}$. The enantiomeric excess was determined by HPLC on Daicel Chiralpak IB-H with hexane/i-PrOH $(95: 5)$ as the eluent, flow $=1.0 \mathrm{~mL} / \mathrm{min}, \mathrm{UV}=254 \mathrm{~nm},[\alpha]_{D}^{25}=-136.000$ (c $=1.0$ in $\left.\mathrm{CH}_{2} \mathrm{Cl}_{2}\right) .{ }^{1} \mathbf{H}$ NMR $\left(600 \mathrm{MHz}, \mathrm{CDCl}_{3}\right) \delta$ 7.44-7.43 (m, 2H), 7.29-7.26 (m, 7H), 7.23-7.18 $(\mathrm{m}, 2 \mathrm{H}), 7.08(\mathrm{td}, J=8.1,5.1 \mathrm{~Hz}, 1 \mathrm{H}), 6.97(\mathrm{ddd}, J=9.7,8.2,1.5 \mathrm{~Hz}, 1 \mathrm{H}), 6.73(\mathrm{~d}, J=7.9 \mathrm{~Hz}, 1 \mathrm{H})$, $5.51(\mathrm{~d}, J=12.6 \mathrm{~Hz}, 1 \mathrm{H}), 4.97(\mathrm{~s}, 1 \mathrm{H}), 4.48(\mathrm{~d}, J=12.6 \mathrm{~Hz}, 1 \mathrm{H}), 1.41(\mathrm{~s}, 9 \mathrm{H}) .{ }^{13} \mathrm{C}$ NMR $(151 \mathrm{MHz}$, $\left.\mathrm{CDCl}_{3}\right) \delta 201.7,169.0,153.9\left(\mathrm{~d},{ }^{1} J_{\mathrm{C}-\mathrm{F}}=249.9 \mathrm{~Hz}\right), 141.2\left(\mathrm{~d},{ }^{3} J_{\mathrm{C}-\mathrm{F}}=12.0 \mathrm{~Hz}\right), 140.2,138.5,136.8$, 129.1, 128.8, 128.6, 128.3, 127.7, $127.2\left(\mathrm{~d},{ }^{3} J_{\mathrm{C}-\mathrm{F}}=7.7 \mathrm{~Hz}\right), 127.0123 .3\left(\mathrm{~d},{ }^{4} J_{\mathrm{C}-\mathrm{F}}=3.6 \mathrm{~Hz}\right), 114.8$ $\left(\mathrm{d},{ }^{2} J_{\mathrm{C}-\mathrm{F}}=18.7 \mathrm{~Hz}\right), 100.0,68.1,42.0,37.7,28.4$. HRMS (ESI) $\mathrm{m} / \mathrm{z}:[\mathrm{M}+\mathrm{Na}]+$ Calcd for $\mathrm{C}_{27} \mathrm{H}_{25} \mathrm{FO}_{2} \mathrm{Na} 423.1731$; Found 421.1737 . 


\section{Scale-up experiment}
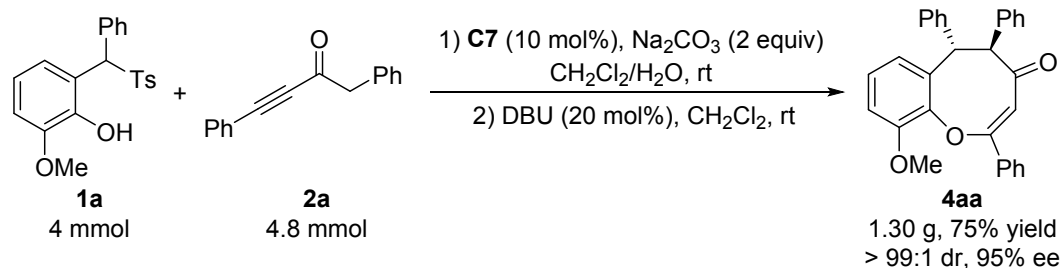

To a test tube were added catalyst $\mathbf{C} 7(0.4 \mathrm{mmol}), \mathrm{Na}_{2} \mathrm{CO}_{3}(8 \mathrm{mmol})$, the substituted 2(phenyl(tosyl)methyl)phenol 1a (4 mmol) and ynones $2 \mathbf{a}(4.8 \mathrm{mmol}), 60 \mathrm{~mL} \mathrm{DCM}$ and $20 \mathrm{~mL} \mathrm{H}_{2} \mathrm{O}$ were then added through syringe. The resulting mixture was stirred at room temperature for $48 \mathrm{~h}$. Then it was extracted with $\mathrm{CH}_{2} \mathrm{Cl}_{2}$, and the organic layer was concentrated under reduced pressure. The resulting residue was directly subjected to flash column chromatography on silica gel (petroleum ether/ethyl acetate $=3: 1$ ) to give the Michael adduct 3aa. The collected intermediates were dissolved in $\mathrm{CH}_{2} \mathrm{Cl}_{2}(40 \mathrm{~mL})$, then DBU $(0.8 \mathrm{mmol})$ was added to the solution and the resulting mixture was stirred at $25{ }^{\circ} \mathrm{C}$ until the reaction was detected complete. The solvent was removed under reduced pressure, the resulting residue was purified by column chromatography on silica gel (petroleum ether/ethyl acetate $=4: 1)$ to give the eight-membered cyclic ether $\mathbf{4 a a}$ in $75 \%$ yield $(1.30$ $\mathrm{g},>99: 1 \mathrm{dr}$, and $95 \%$ ee).

\section{Synthetic transformations of products}

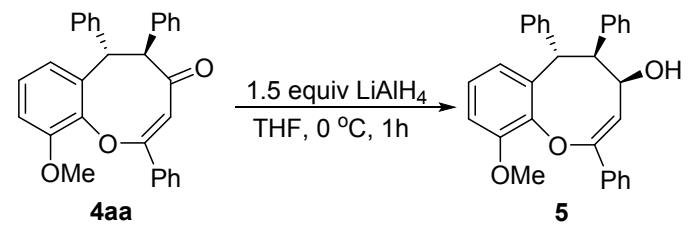

The 4 aa $(0.1 \mathrm{mmol})$ was dissolved in $2 \mathrm{~mL}$ of THF, cooled to $0{ }^{\circ} \mathrm{C}$ and $\mathrm{LiAlH}_{4}(0.15 \mathrm{mmol})$ was added slowly. The mixture was stirred at room temperature for 1 hour. After the reaction was complete, a little water was added to quench the reaction, and the organic product was extracted with ethyl acetate $(10 \mathrm{~mL} \times 3)$. The residue was purified by column chromatography on silica gel with EtOAc/petroleum ether $(1 / 3)$ as the eluent to give 5. Purified by silica gel chromatography using PE/EA 3:1, white solid, mp 148-150 ${ }^{\circ} \mathrm{C}, 95 \%$ yield, $41.2 \mathrm{mg}, 94 \%$ ee, $>99: 1 \mathrm{dr}$. The enantiomeric excess was determined by HPLC on Daicel Chiralpak IB-H with hexane/i-PrOH (90:10) as the eluent, flow $=1.0 \mathrm{~mL} / \mathrm{min}, \mathrm{UV}=254 \mathrm{~nm},[\alpha]_{D}^{25}=-125.000\left(\mathrm{c}=1.0\right.$ in $\mathrm{CH}_{2} \mathrm{Cl}_{2}$ ). ${ }^{1} \mathbf{H}$ NMR $\left(500 \mathrm{MHz}, \mathrm{CDCl}_{3}\right) \delta 7.93(\mathrm{~d}, J=7.2 \mathrm{~Hz}, 2 \mathrm{H}), 7.48(\mathrm{dd}, J=13.8,7.3 \mathrm{~Hz}, 4 \mathrm{H}), 7.43-7.41$ (m, 1H), 7.33-7.30 (m, 4H), 7.28-7.20 (m, 4H), 7.14-7.09 (m, 2H), $6.89(\mathrm{dd}, J=8.1,1.0 \mathrm{~Hz}, 1 \mathrm{H})$, $6.56(\mathrm{~d}, J=7.8 \mathrm{~Hz}, 1 \mathrm{H}), 5.35(\mathrm{~d}, J=12.2 \mathrm{~Hz}, 1 \mathrm{H}), 4.92(\mathrm{~d}, J=5.1 \mathrm{~Hz}, 1 \mathrm{H}), 4.54(\mathrm{~s}, 1 \mathrm{H}), 3.94(\mathrm{~s}$, $3 \mathrm{H}), 3.71(\mathrm{dd}, J=12.2,5.0 \mathrm{~Hz}, 1 \mathrm{H}) .{ }^{13} \mathrm{C}$ NMR $\left(126 \mathrm{MHz}, \mathrm{CDCl}_{3}\right) \delta 152.0,150.9,142.1,140.4$, 138.3, 137.7, 137.1, 129.9, 129.3, 128.5, 128.4, 128.4, 128.2, 127.4, 126.4, 126.3, 126.1, 120.0, 110.8, 107.5, 68.5, 56.2, 55.1, 44.8. HRMS (ESI) m/z: [M + Na]+ Calcd for $\mathrm{C}_{30} \mathrm{H}_{26} \mathrm{O}_{3} \mathrm{Na}$ 457.1774; Found 457.1781.

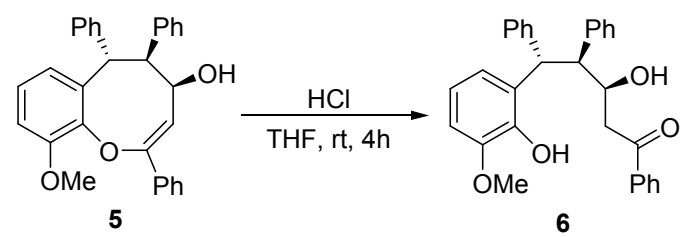


The $5(0.1 \mathrm{mmol})$ was dissolved in $2 \mathrm{~mL}$ of THF, $1 \mathrm{~mL} \mathrm{HCl}(4 \mathrm{M})$ were added and the reaction was stirred $3 \mathrm{~h}$ at rt. After the reaction was complete, the organic product was extracted with $\mathrm{CH}_{2} \mathrm{Cl}_{2}$ $(10 \mathrm{~mL} \times 3)$. The residue was purified by column chromatography on silica gel with EtOAc/petroleum ether $(1 / 5)$ as the eluent to give 6. Purified by silica gel chromatography using PE/EA 5:1, colorless oil, 78\% yield, $35.2 \mathrm{mg}, 88 \%$ ee, $>99: 1 \mathrm{dr}$. The enantiomeric excess was determined by HPLC on Daicel Chiralpak ID-H with hexane/i-PrOH (80:20) as the eluent, flow = $1.0 \mathrm{~mL} / \mathrm{min}, \mathrm{UV}=254 \mathrm{~nm},[\alpha]_{D}^{25}=-47.000\left(\mathrm{c}=1.0\right.$ in $\left.\mathrm{CH}_{2} \mathrm{Cl}_{2}\right) .{ }^{1} \mathbf{H} \mathbf{~ N M R}\left(500 \mathrm{MHz}, \mathrm{CDCl}_{3}\right) \delta$ 7.81-7.79 (m, 2H), 7.56-7.53 (m, 1H), 7.42-7.36 (m, 4H), 7.32 (d, $J=7.4 \mathrm{~Hz}, 2 \mathrm{H}), 7.28-7.23(\mathrm{~m}$, $3 \mathrm{H}), 7.15-7.06(\mathrm{~m}, 4 \mathrm{H}), 6.98(\mathrm{t}, J=7.4 \mathrm{~Hz}, 1 \mathrm{H}), 6.84(\mathrm{t}, J=8.0 \mathrm{~Hz}, 1 \mathrm{H}), 6.70(\mathrm{dd}, J=8.0,1.2 \mathrm{~Hz}$, $1 \mathrm{H}), 6.03(\mathrm{~s}, 1 \mathrm{H}), 5.20(\mathrm{~d}, J=12.5 \mathrm{~Hz}, 1 \mathrm{H}), 4.43-4.41(\mathrm{~m}, 1 \mathrm{H}), 4.13(\mathrm{dd}, J=12.5,4.1 \mathrm{~Hz}, 1 \mathrm{H}), 3.85$ (s, 1H), 3.53 (dd, $J=16.7,1.9 \mathrm{~Hz}, 1 \mathrm{H}), 2.70(\mathrm{dd}, J=16.7,10.4 \mathrm{~Hz}, 1 \mathrm{H}) .{ }^{13} \mathbf{C}$ NMR $(126 \mathrm{MHz}$, $\left.\mathrm{CDCl}_{3}\right) \delta 201.1,146.6,143.1,142.6,138.9,136.9,133.2,130.2,129.3,128.6,128.5,128.1,128.0$, 127.9, 126.5, 125.7, 120.6, 120.4, 108.5, 69.3, 55.9, 53.4, 43.1, 41.2. HRMS (ESI) m/z: [M + Na]+ Calcd for $\mathrm{C}_{30} \mathrm{H}_{28} \mathrm{O}_{4} \mathrm{Na}$ 475.1880; Found 475.1891.

\section{ESI-MS studies}

The addition adduct 3aa $(21.6 \mathrm{mg}, 0.05 \mathrm{mmol})$ were dissolved in methanol $(1 \mathrm{~mL})$, then DBU (20 mol \%) was added to the solution and the resulting mixture was stirred at room temperature for 5 min. then diluted by methanol and subjected directly to ESI-MS analysis. The spectrum was shown in Figure S1. The intermediate $(\mathrm{I} \rightleftharpoons \mathrm{II} \rightleftharpoons \mathrm{III} \rightleftharpoons \mathrm{IV} \rightleftharpoons \mathrm{V})$ was detected at $\mathrm{m} / \mathrm{z}=585.3\left([\mathrm{I}+\mathrm{H}]^{+}\right)$.

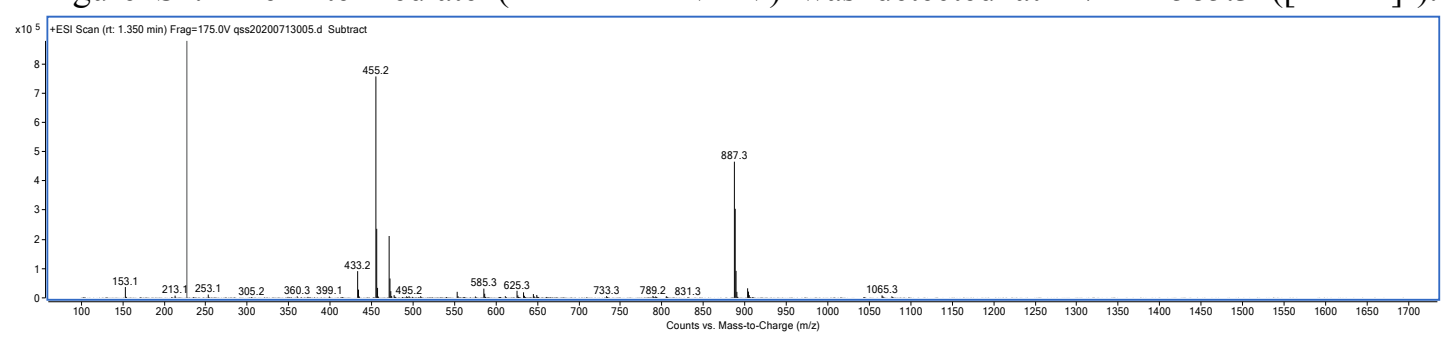

Figure S1. ESI-MS spectrum of the diluted reaction mixture

\section{References}

[1] (a) M-W. Chen, L-L. Cao, Z-S. Ye, G-F. Jiang, and Y-G. Zhou, Chem. Commun. 2013, 49, 1660-1662. (b) M-M. Chu, X-Y. Chen, Y-F. Wang, S-S. Qi, Z-H. Jiang, D-Q. Xu, and Z-Y. Xu, J. Org. Chem. 2020, 85, 9491-9502

[2] (a) X.-F. Wu, H. Neumann and M. Beller. Org. Biomol. Chem., 2011, 9, 8003-8005. (b) Feng, X.; Song, J.; Liu, H.; Wang, L.; Yu, X.; Bao M. RSC. Adv. 2013, 3, 18985-18991. (c) Campano, T. E.; Iriarte, I.; Olaizola, O.; Etxabe, Julen.; Mielgo, A.; Ganboa, I.; Odriozola, J. M.; Garcia, J. M.; Oiarbide, M.; Palomo, C. Chem. Eur. J. 2019, 25, 4390-4397.

\section{HPLC spectra of products}



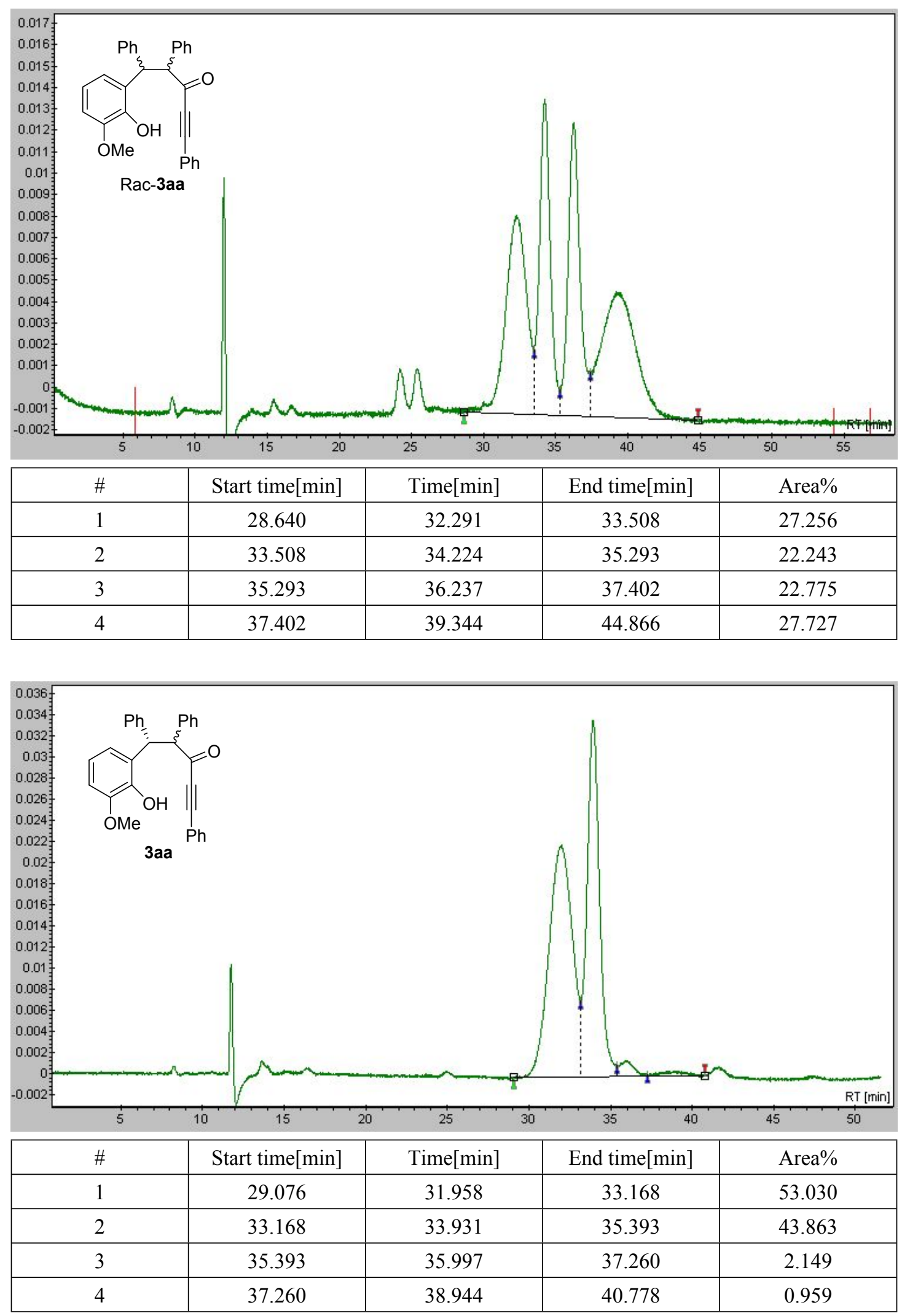

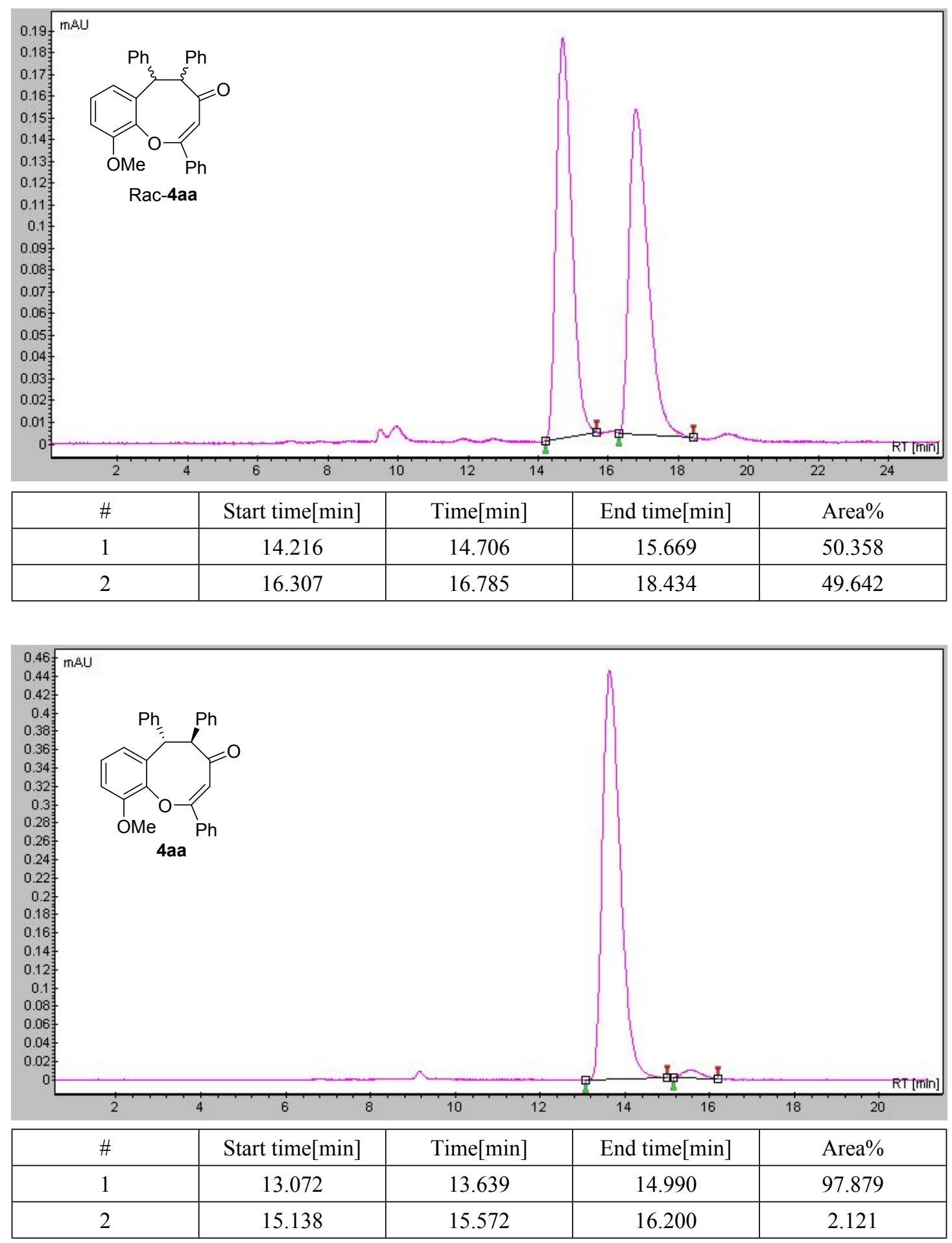

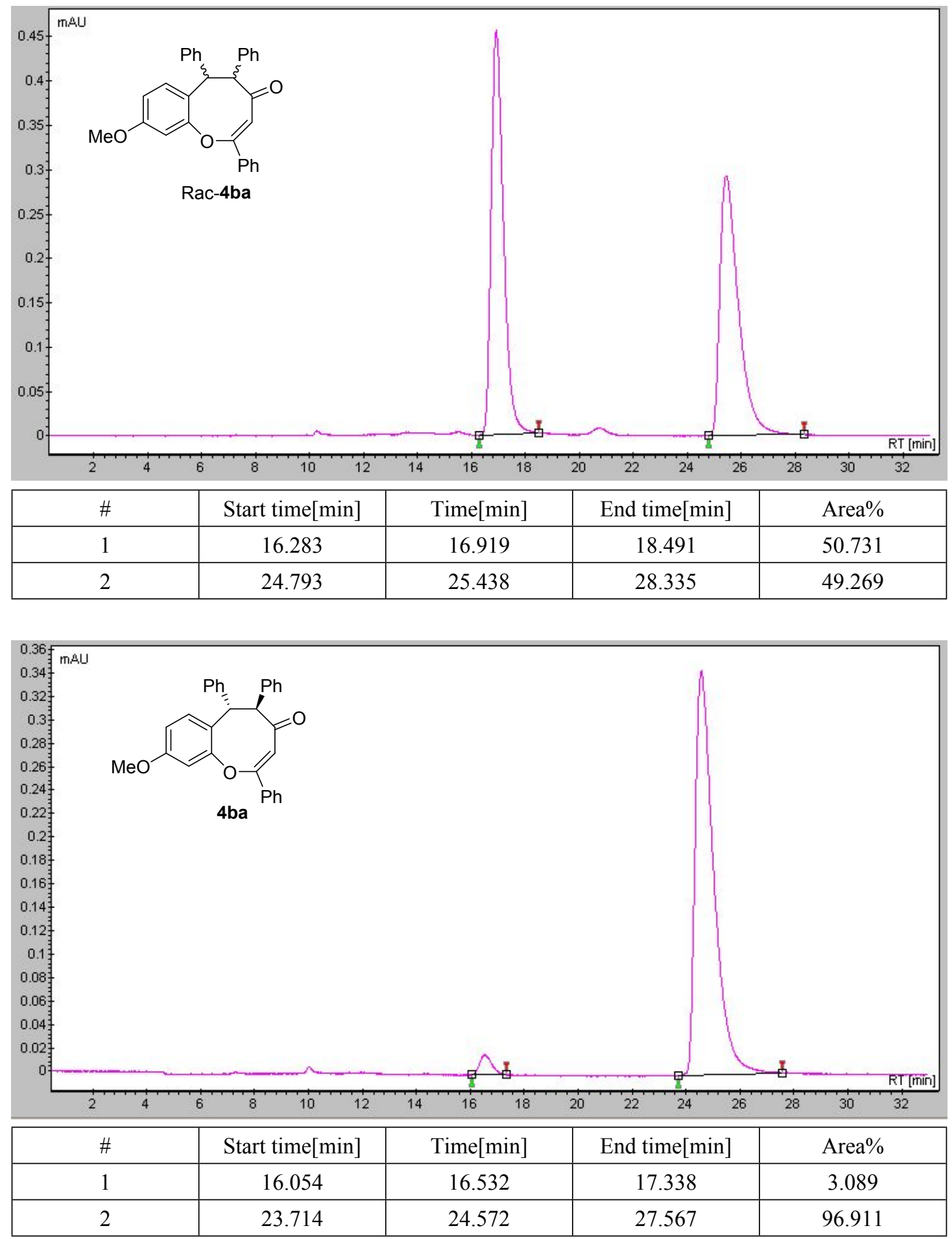

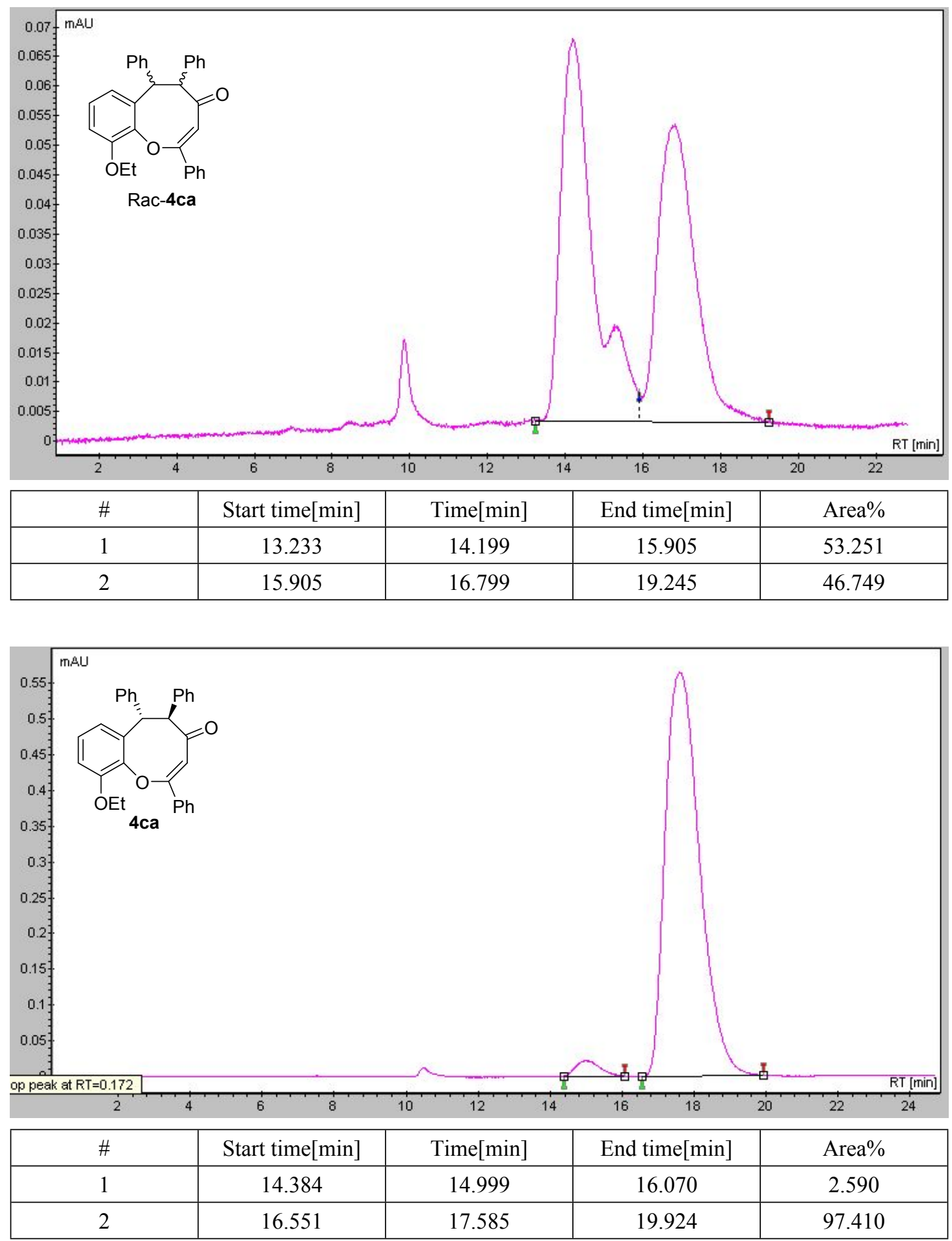

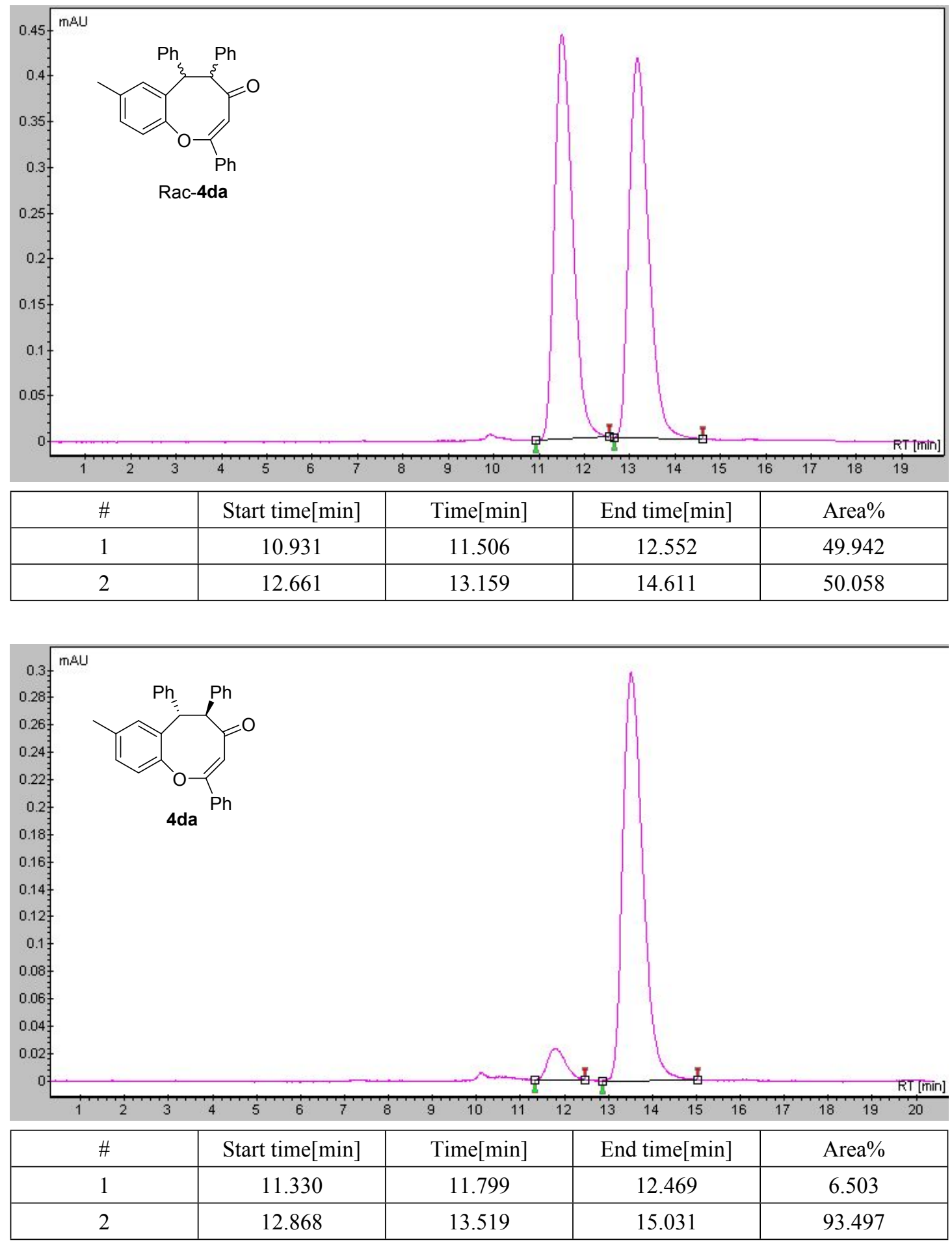

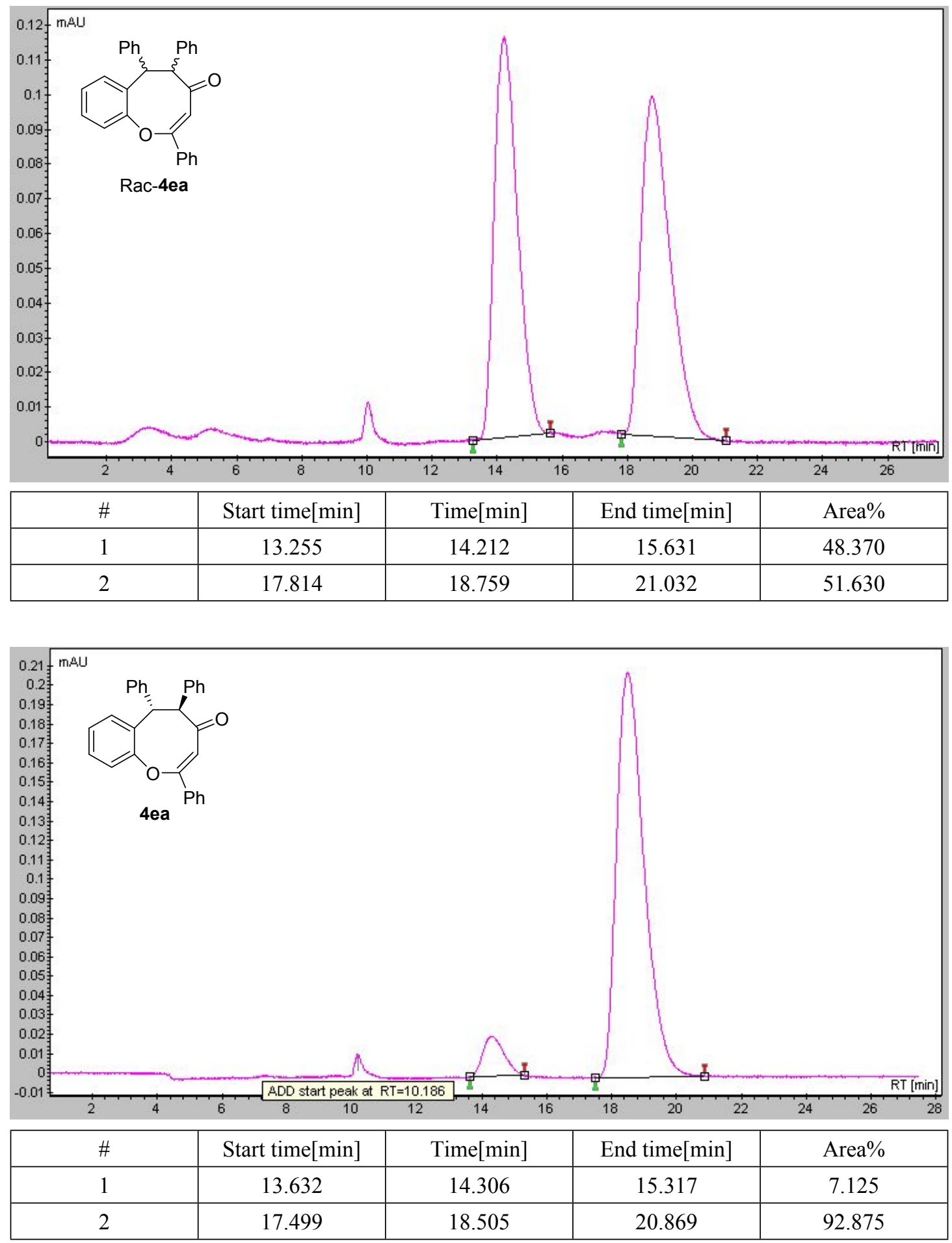

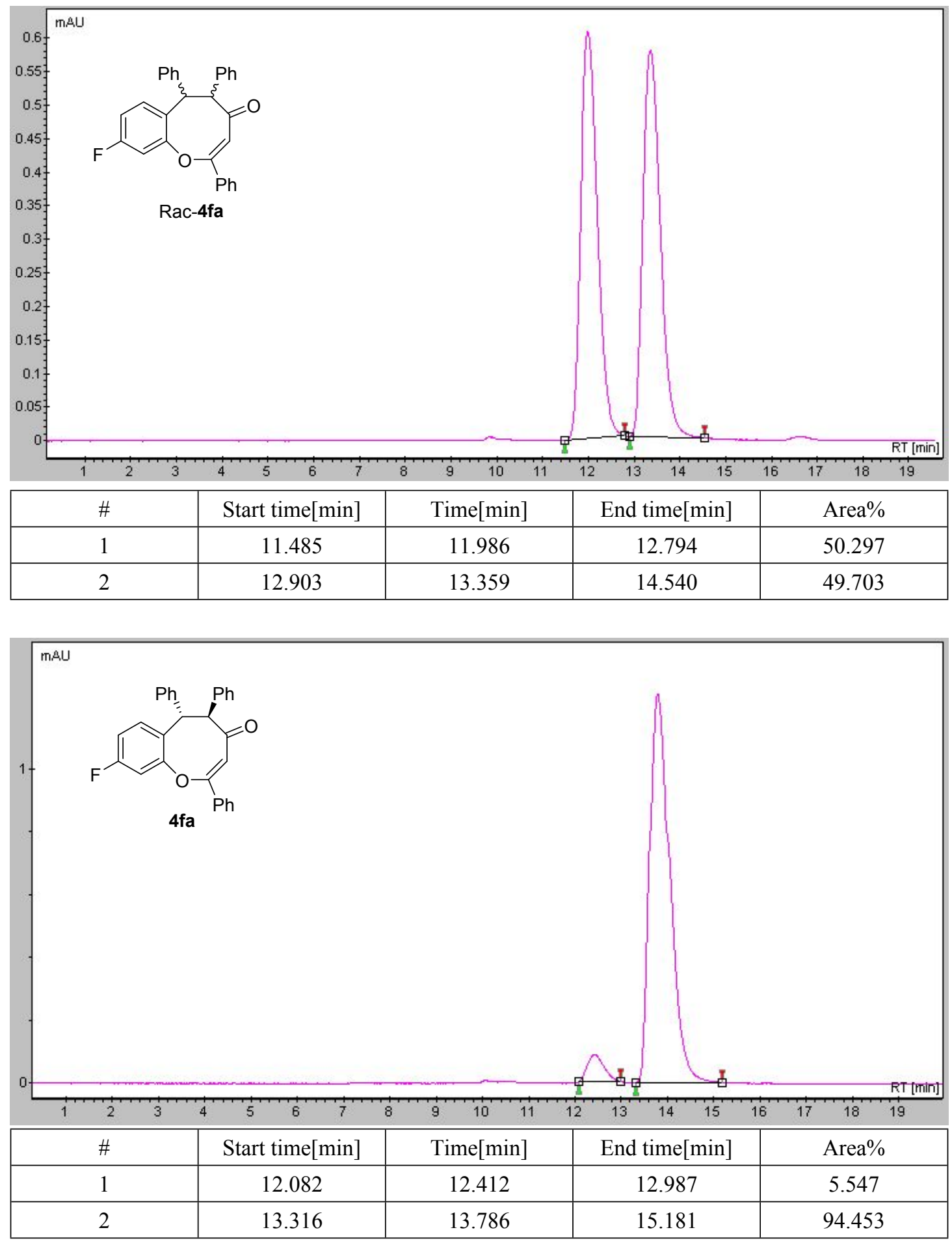

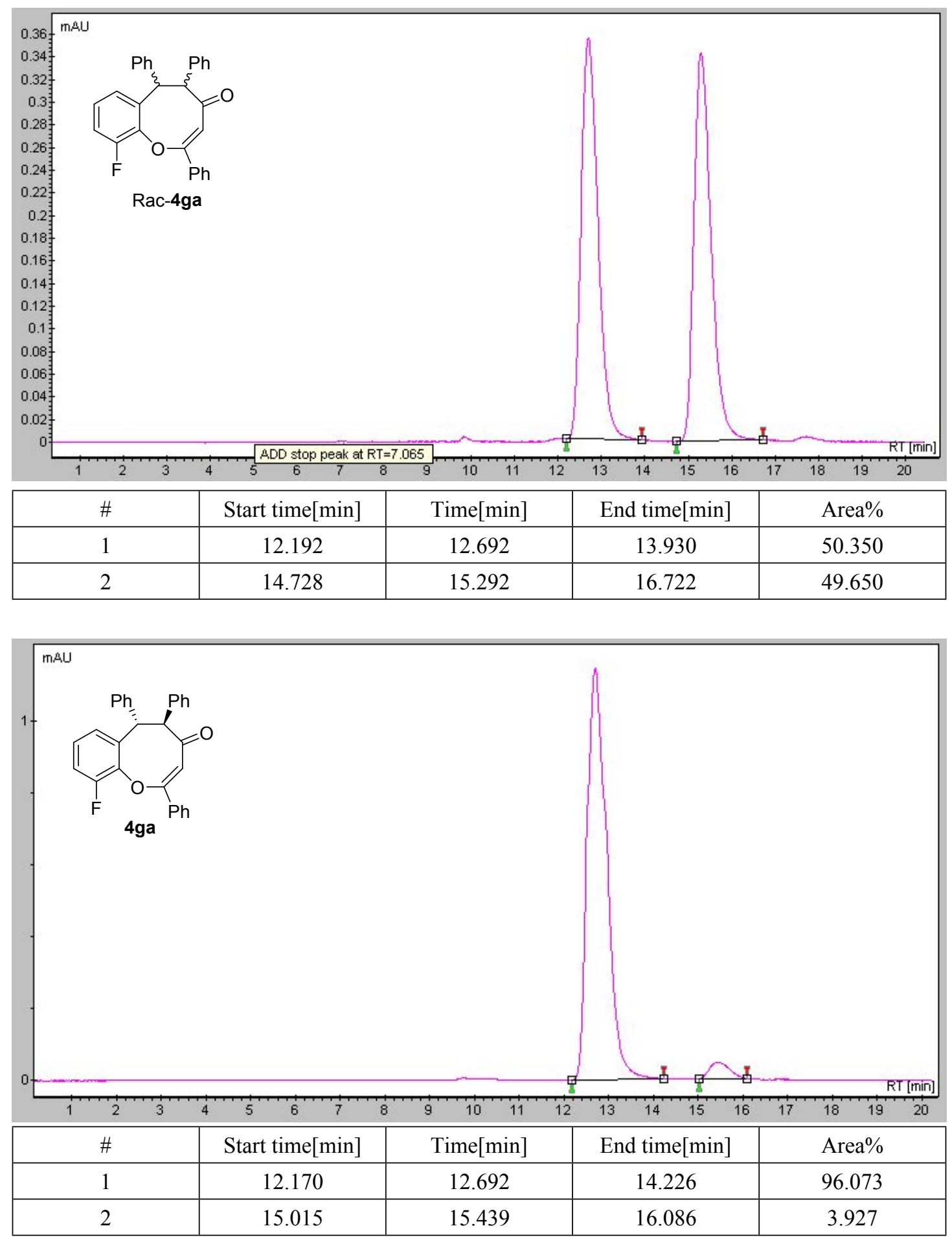

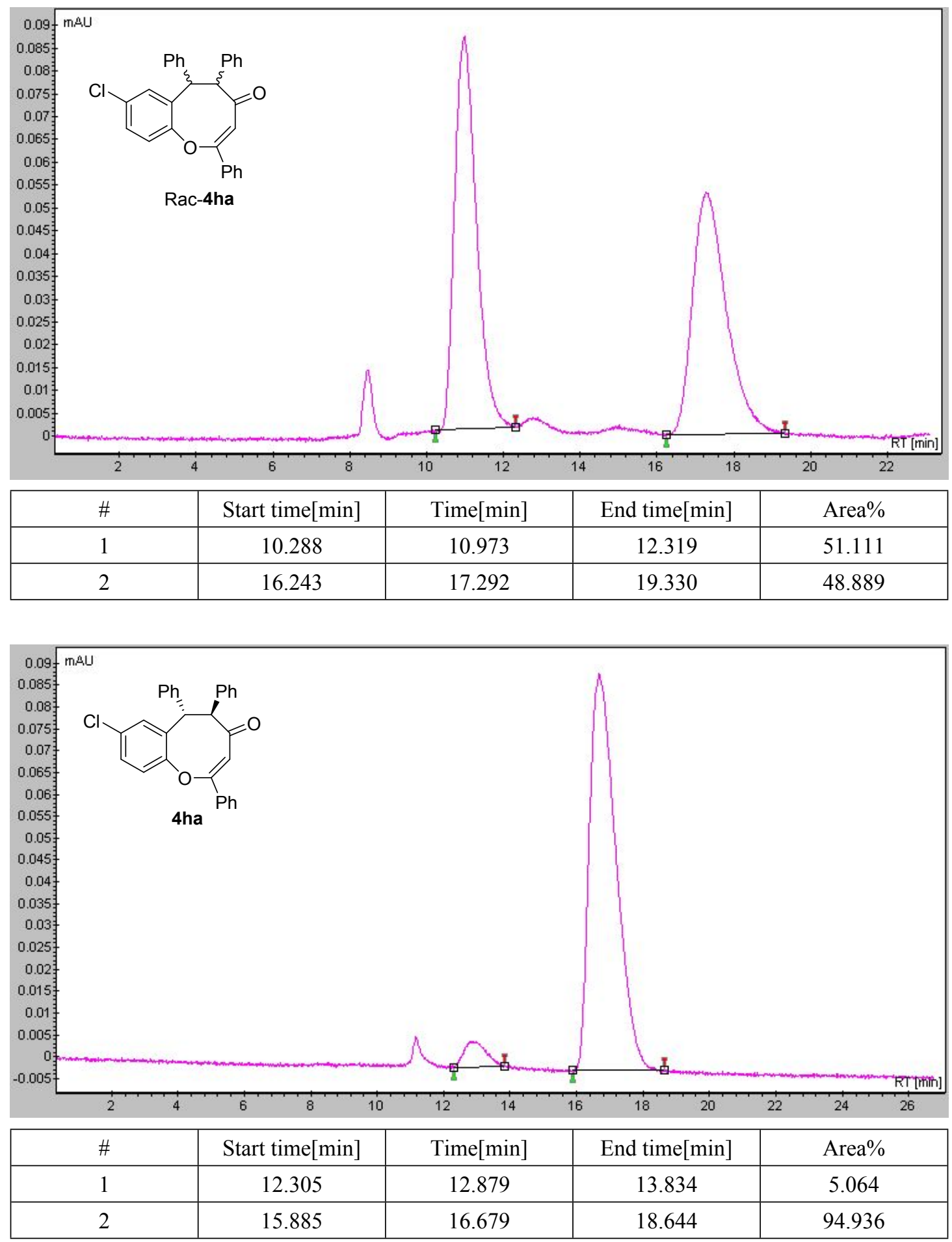

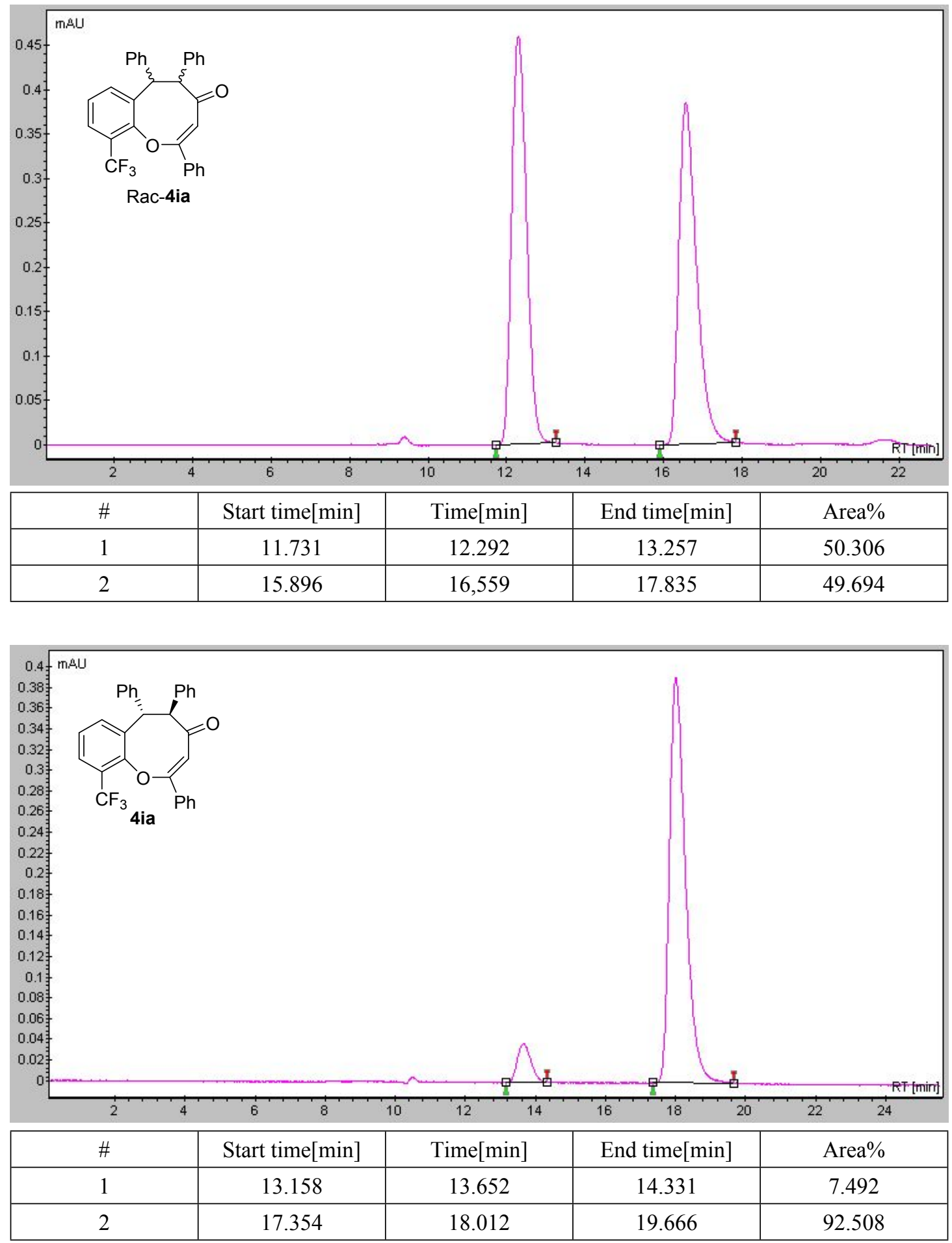

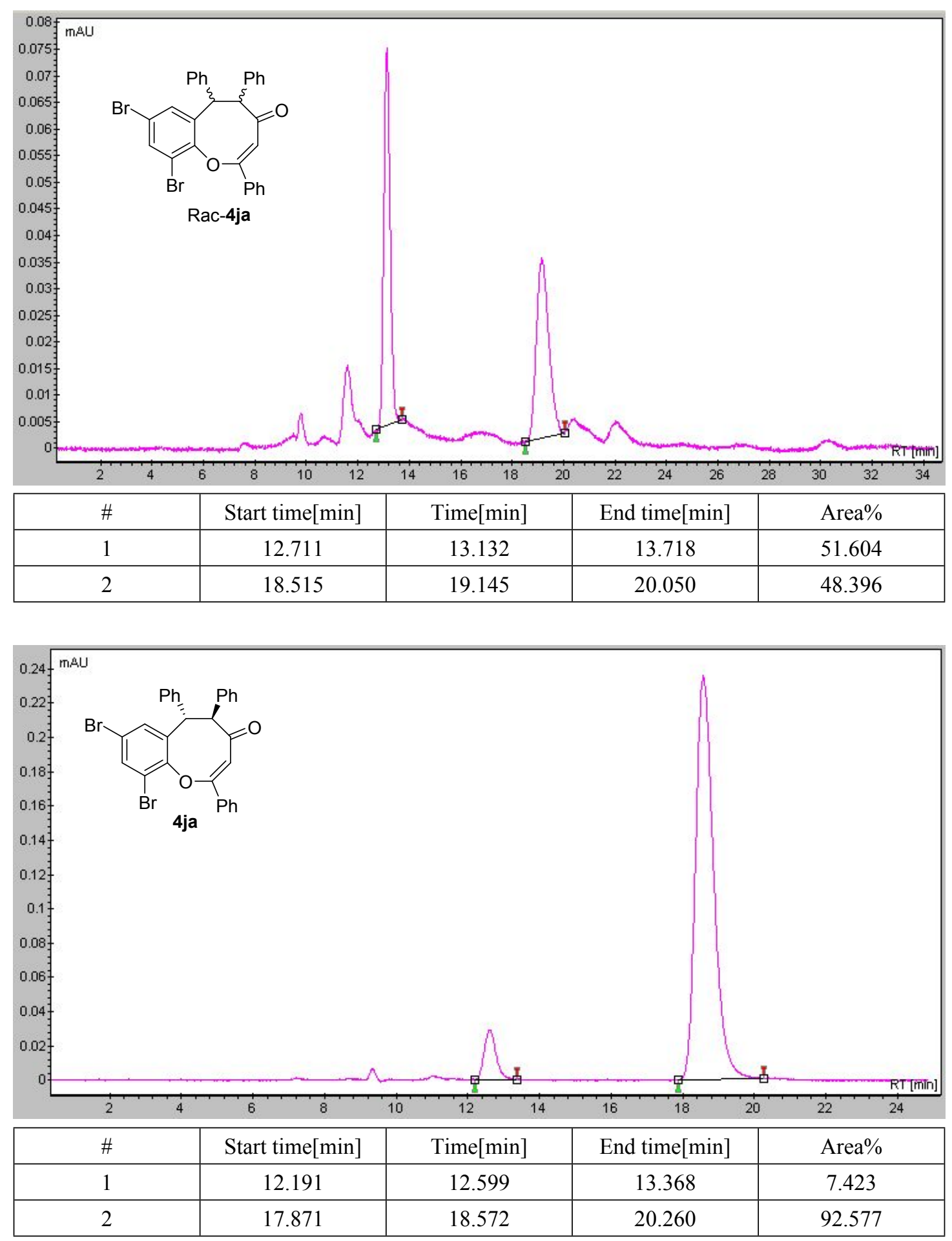

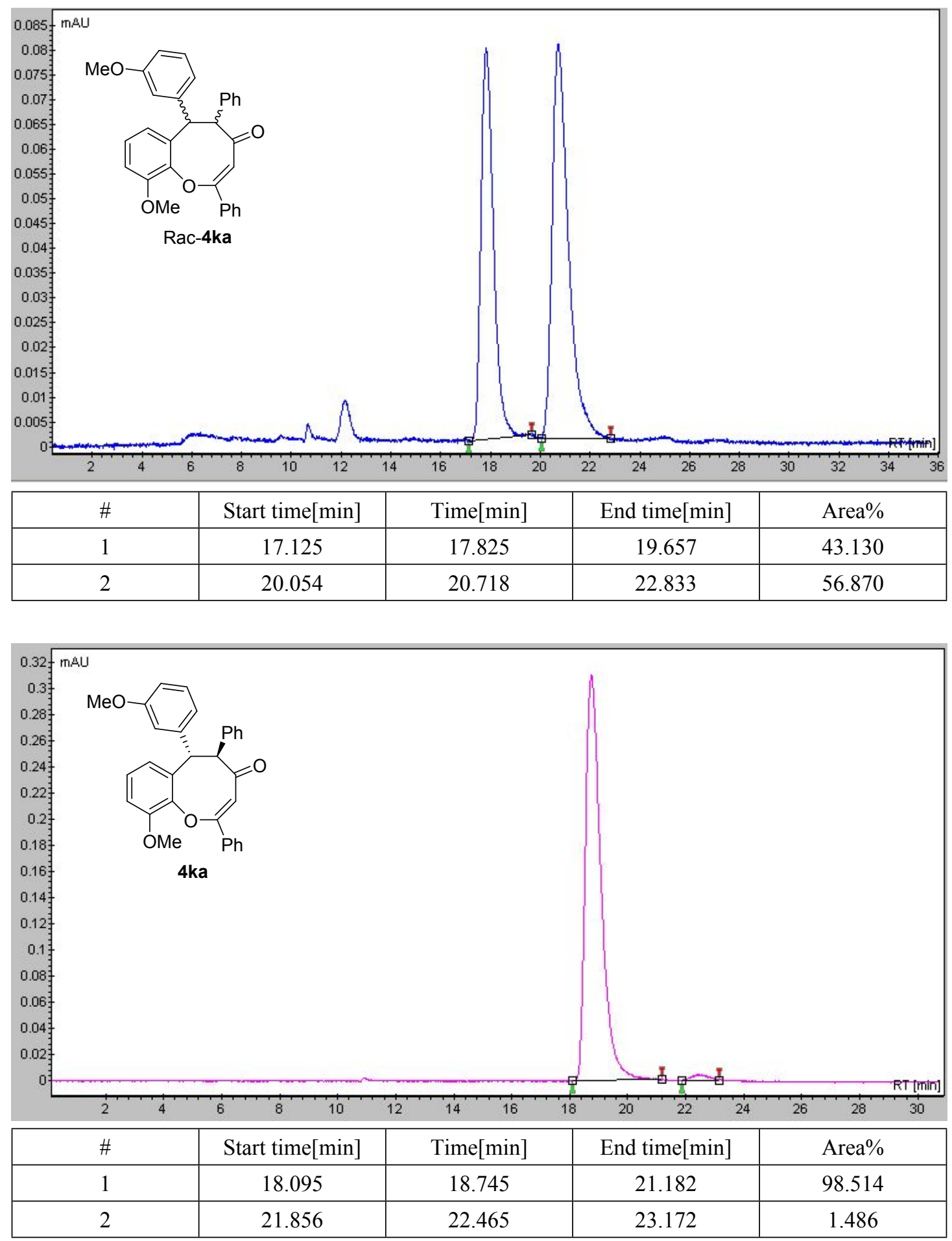

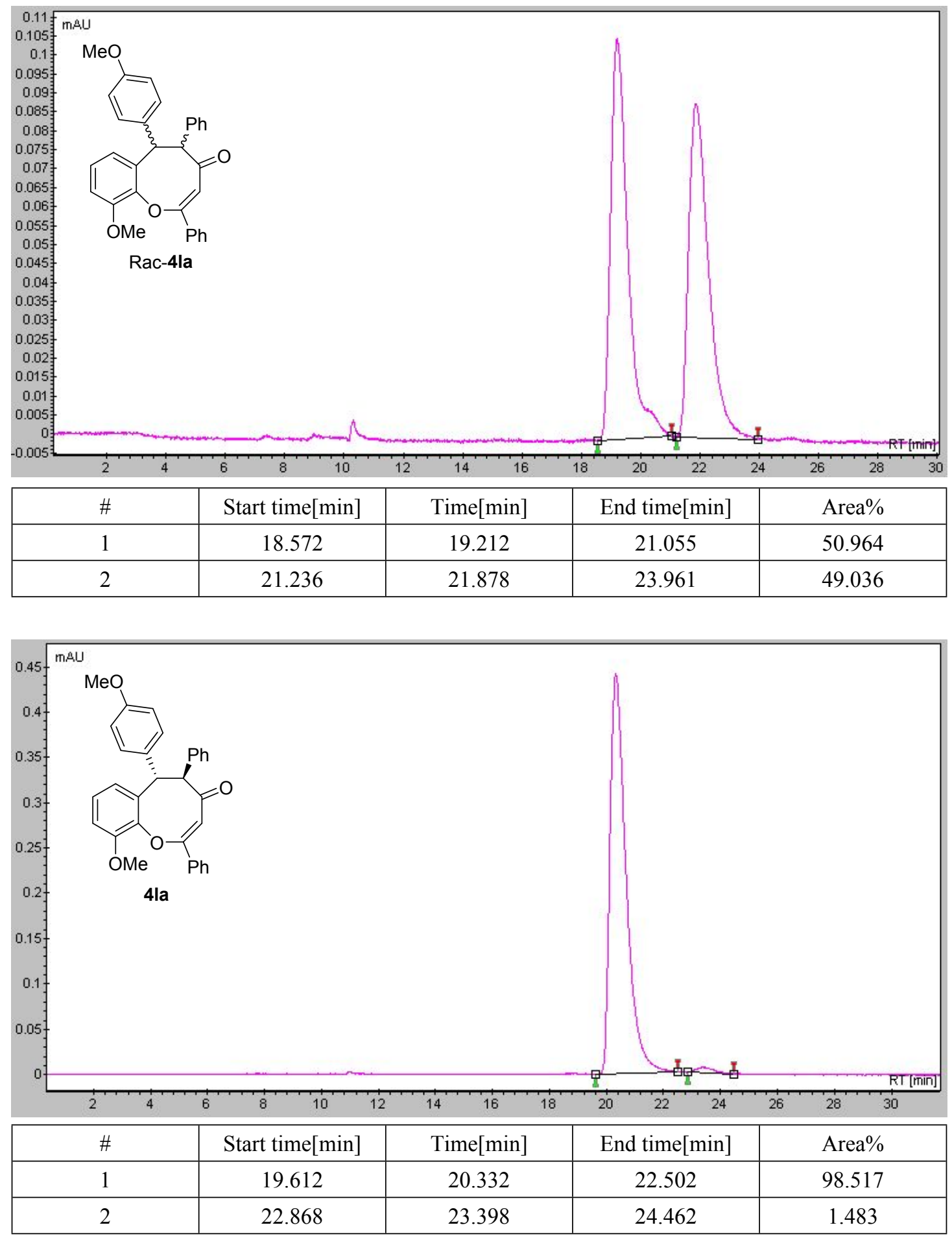

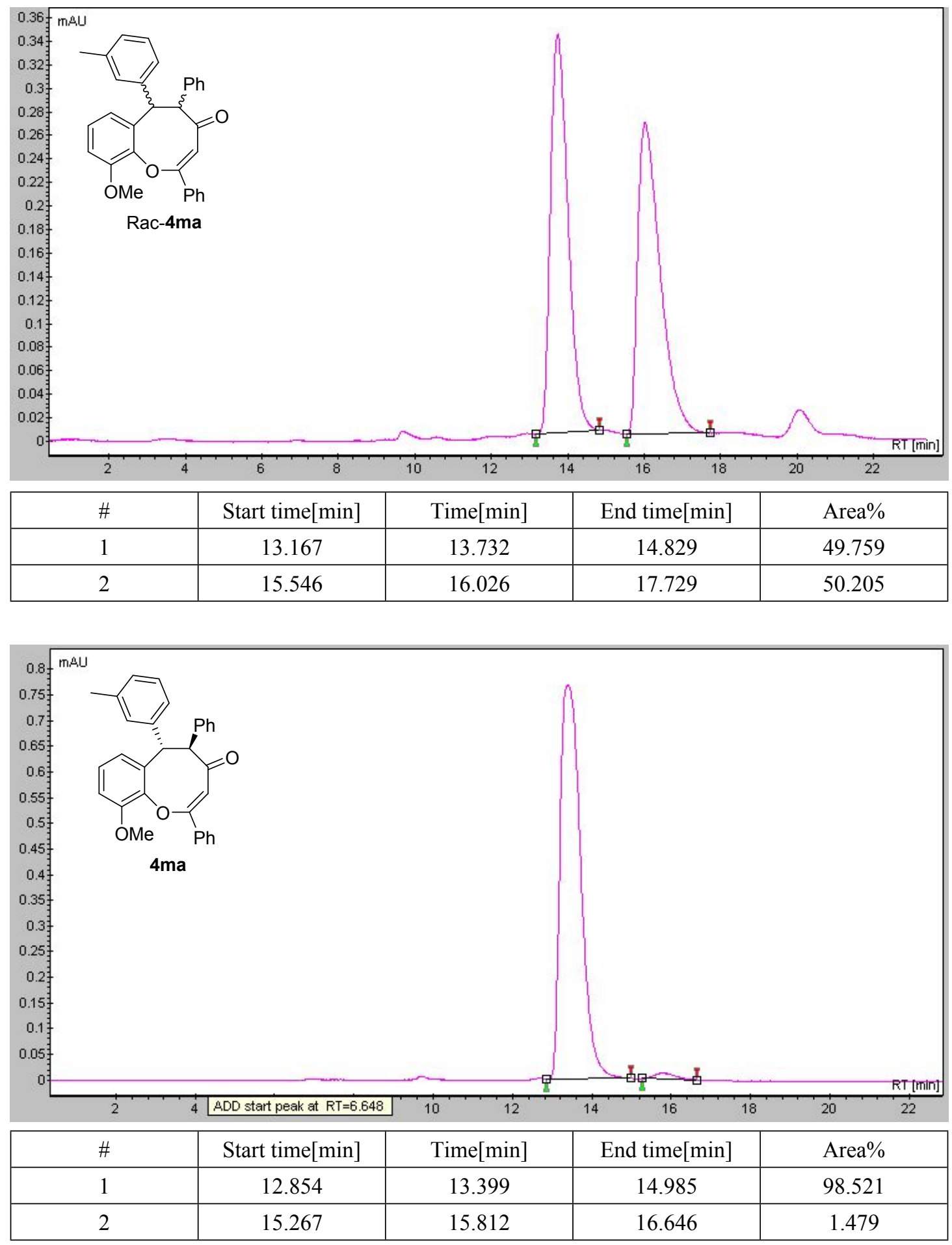

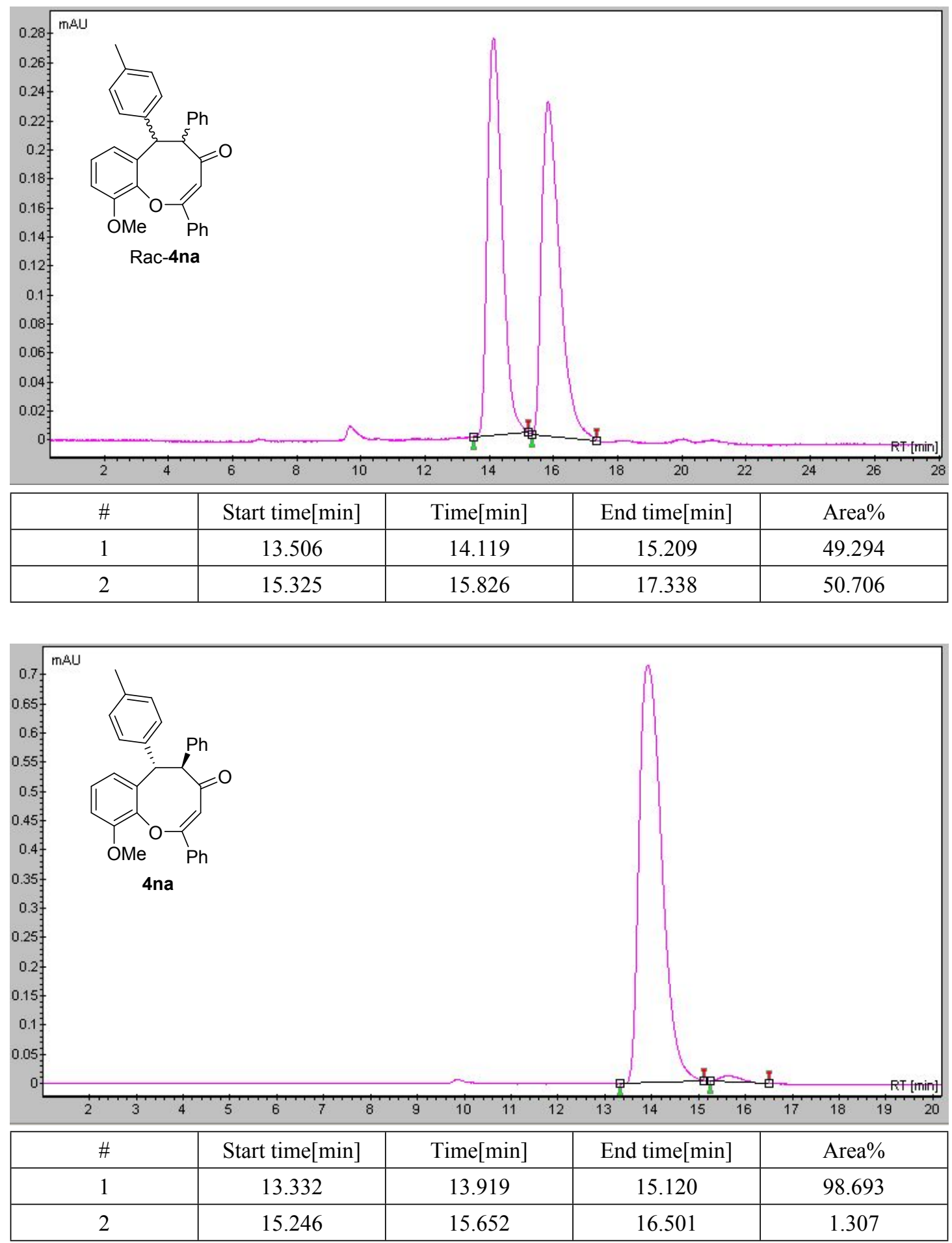

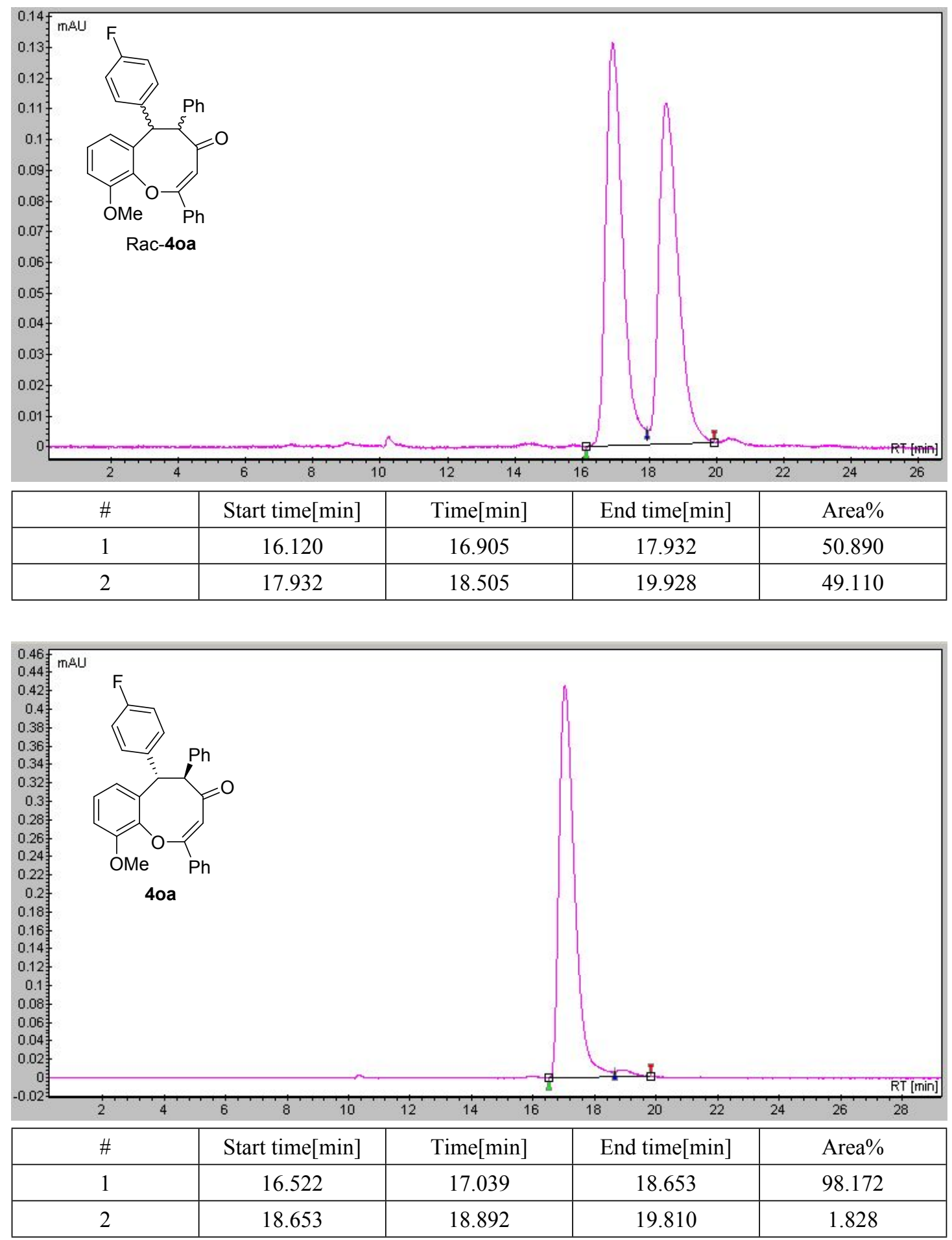

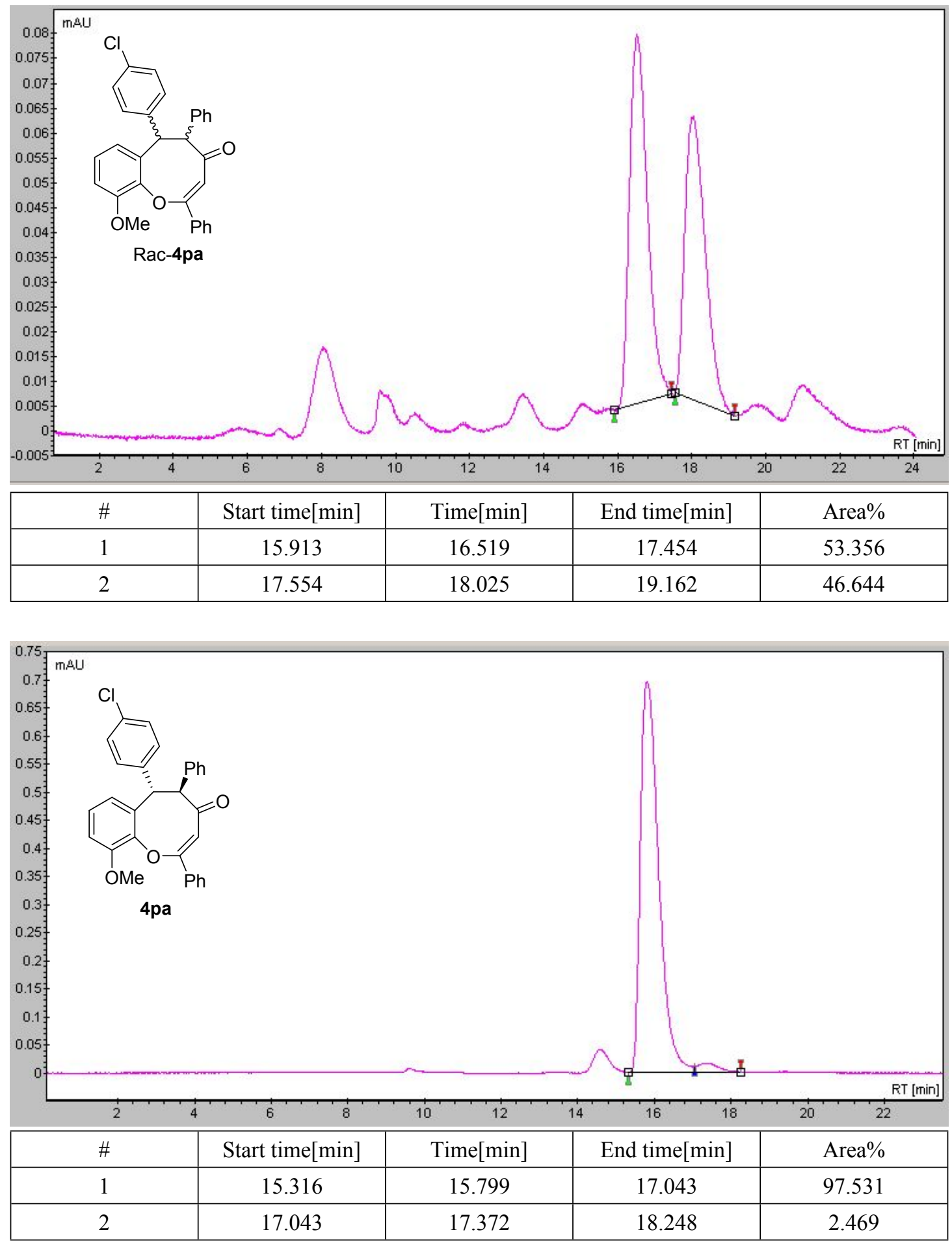

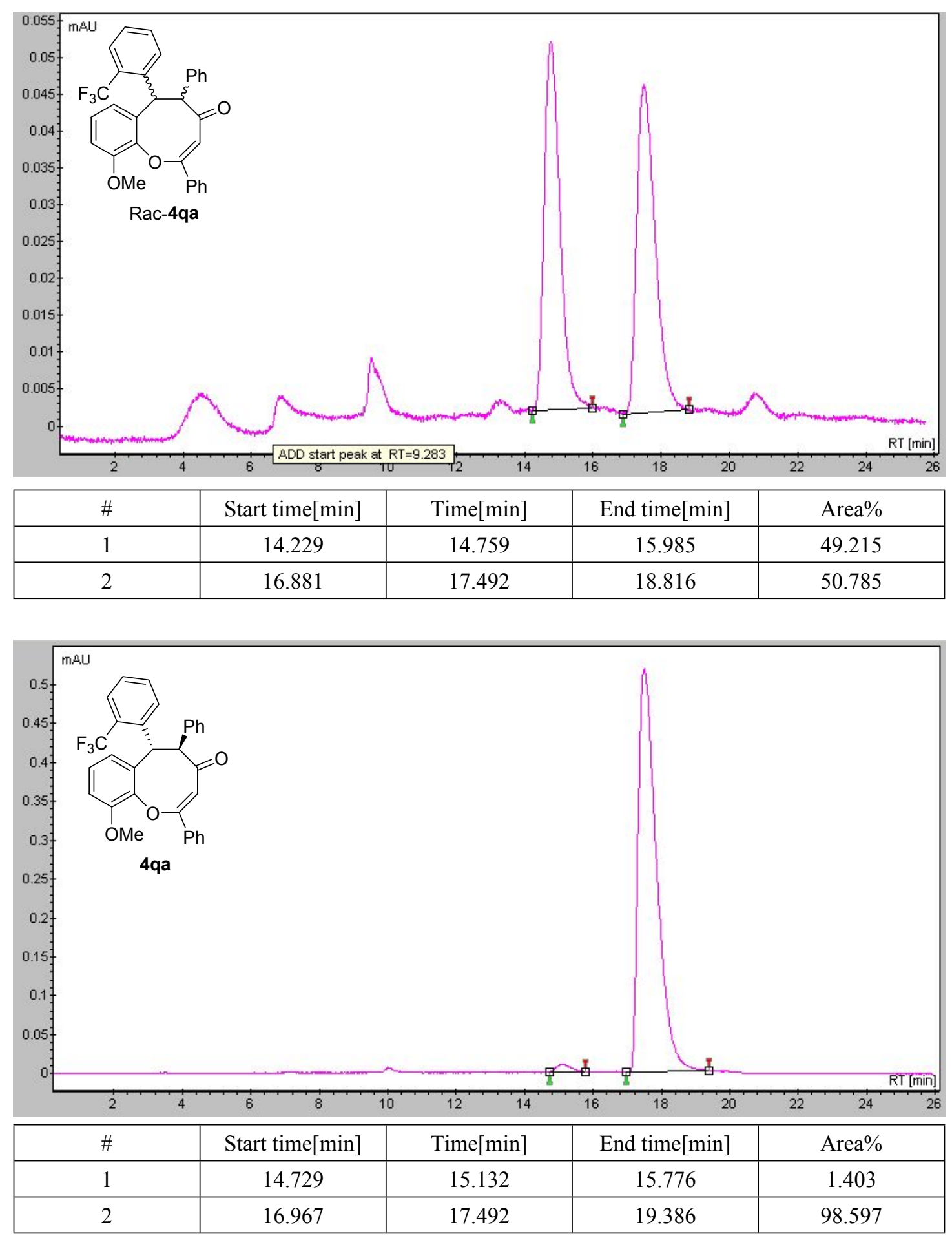

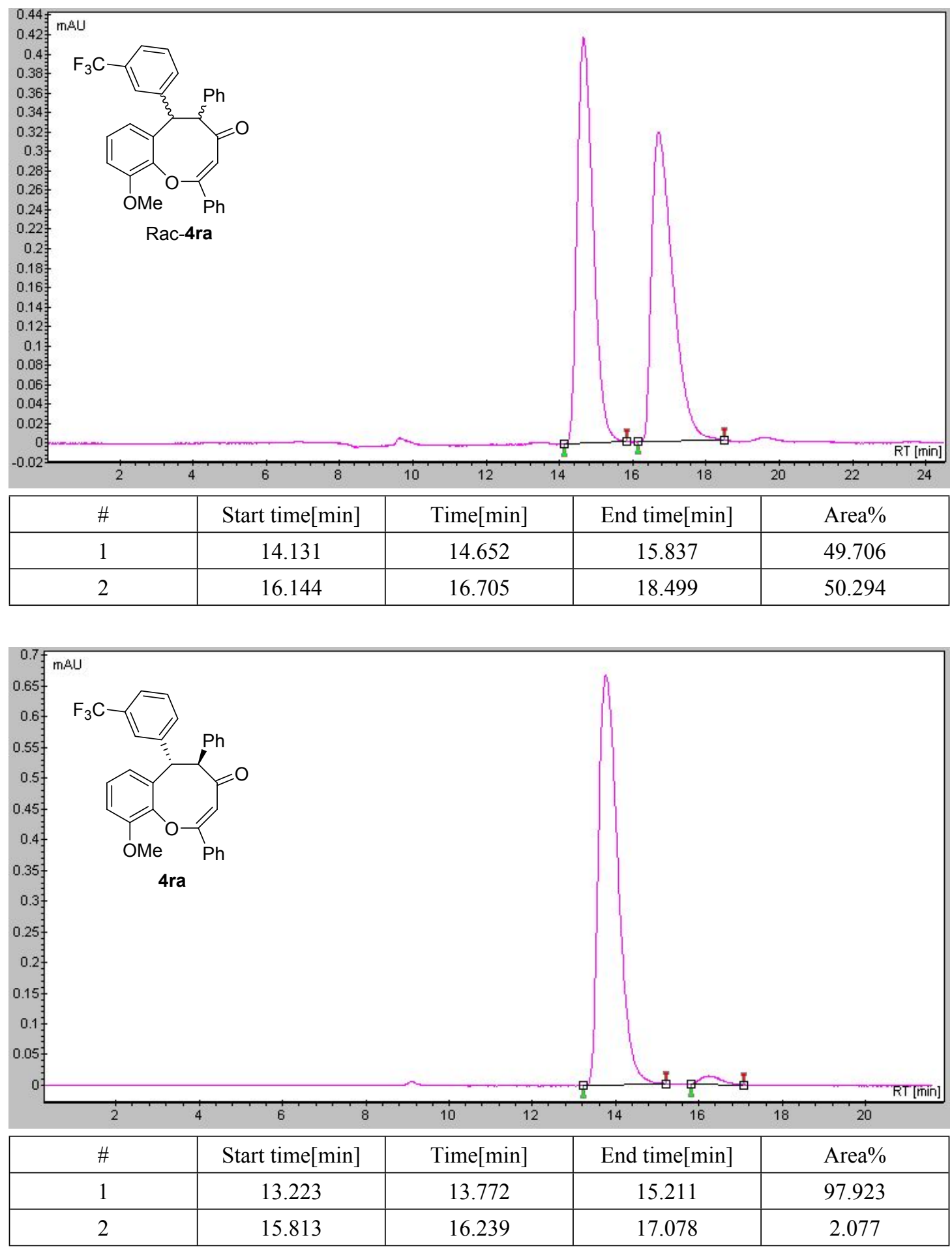

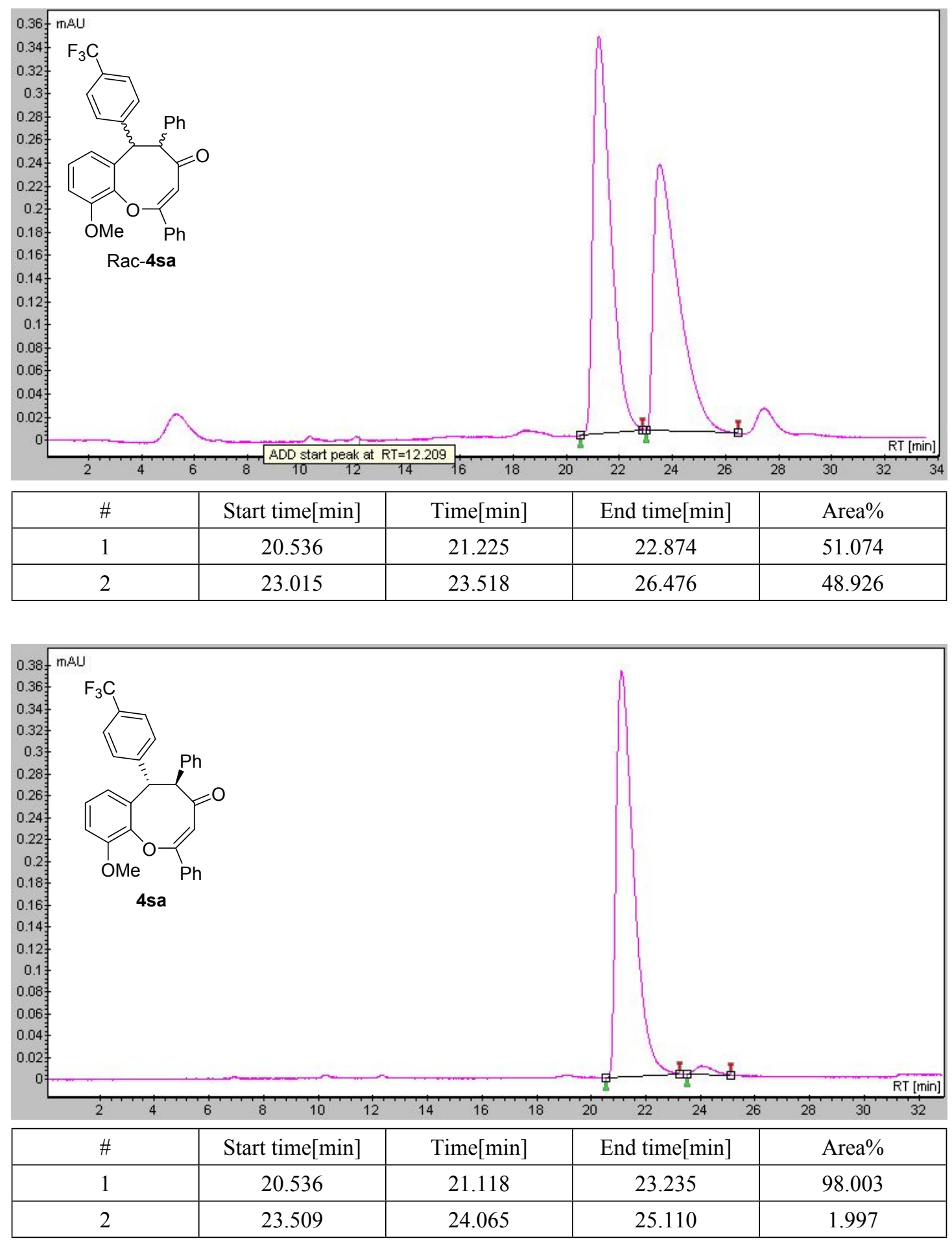

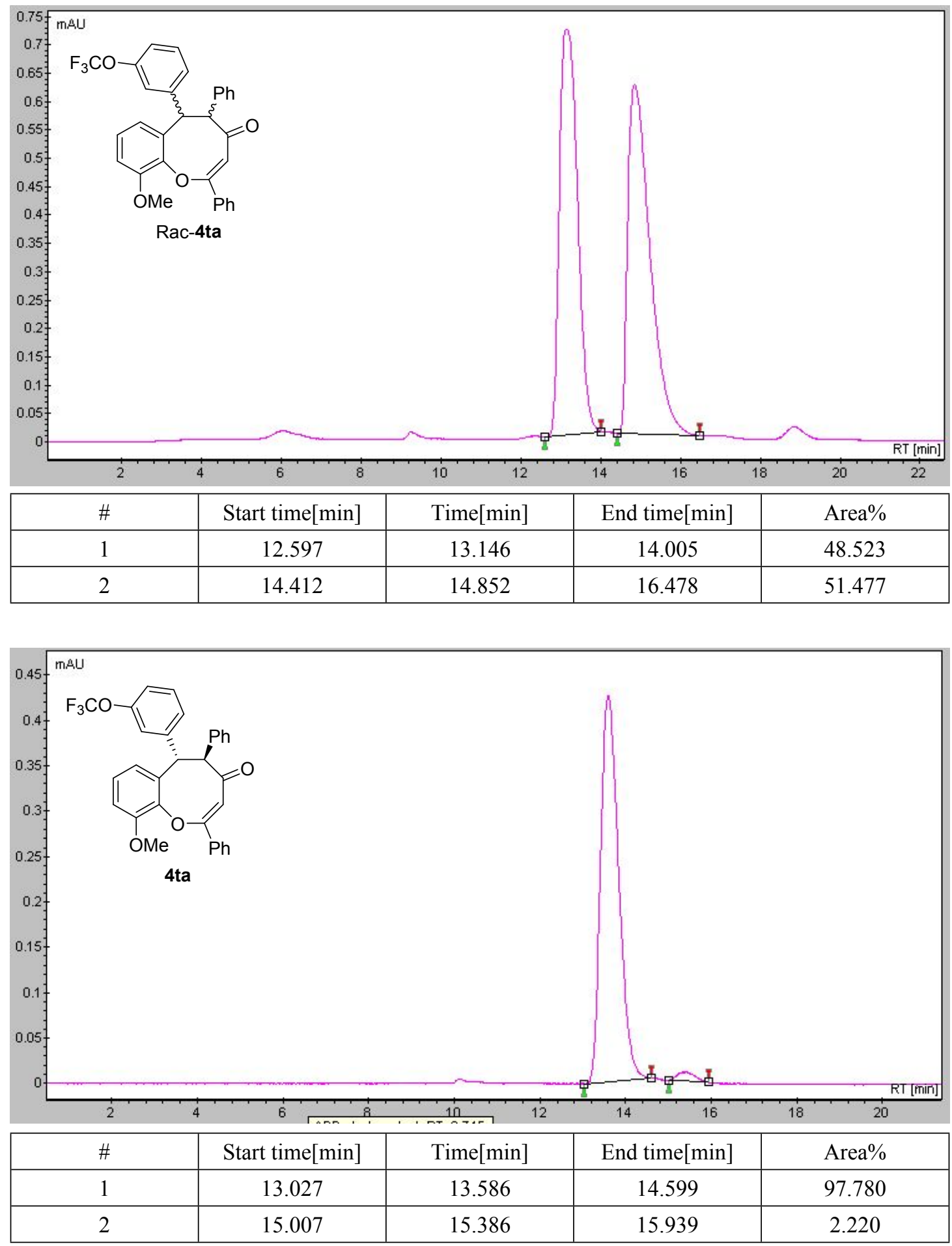

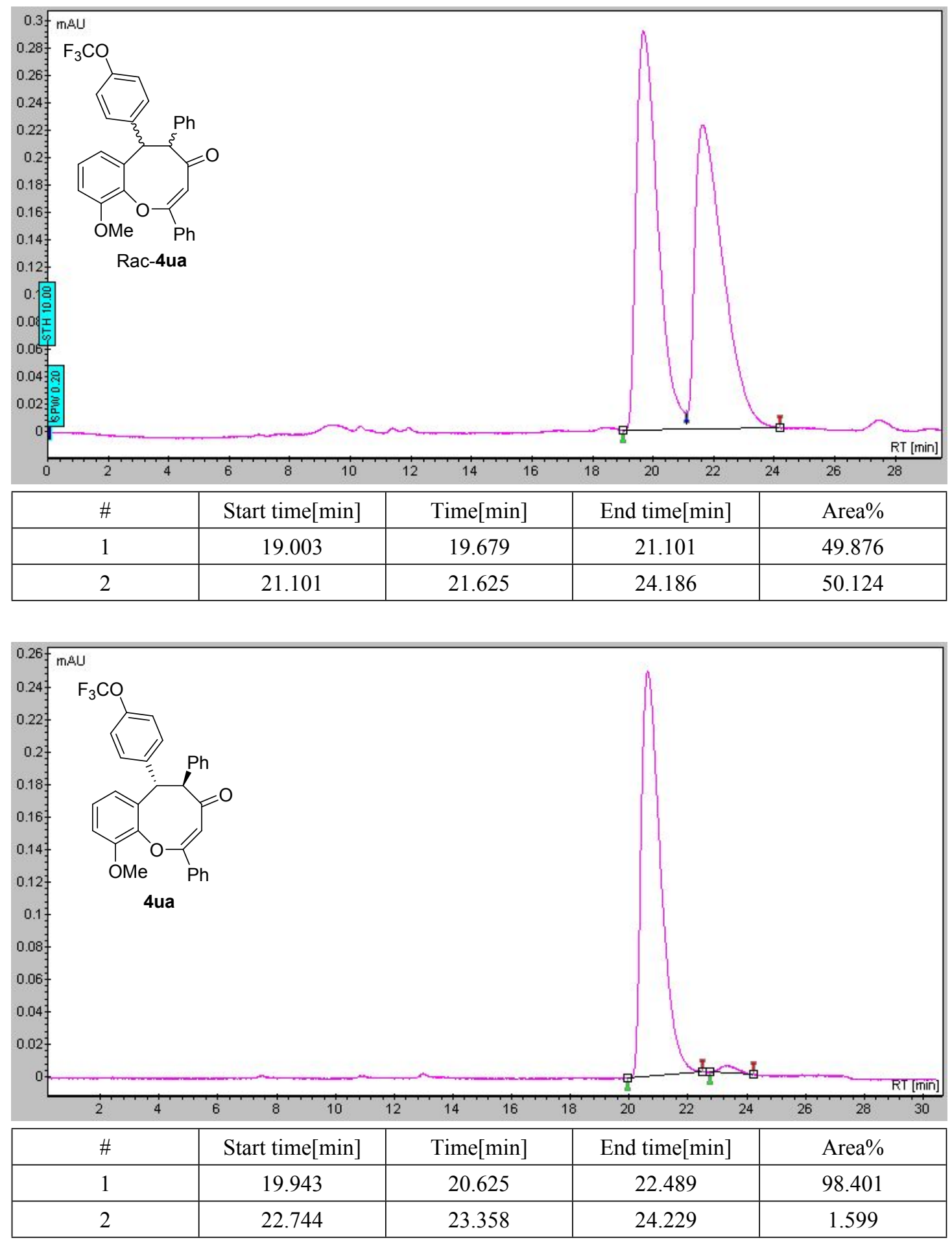

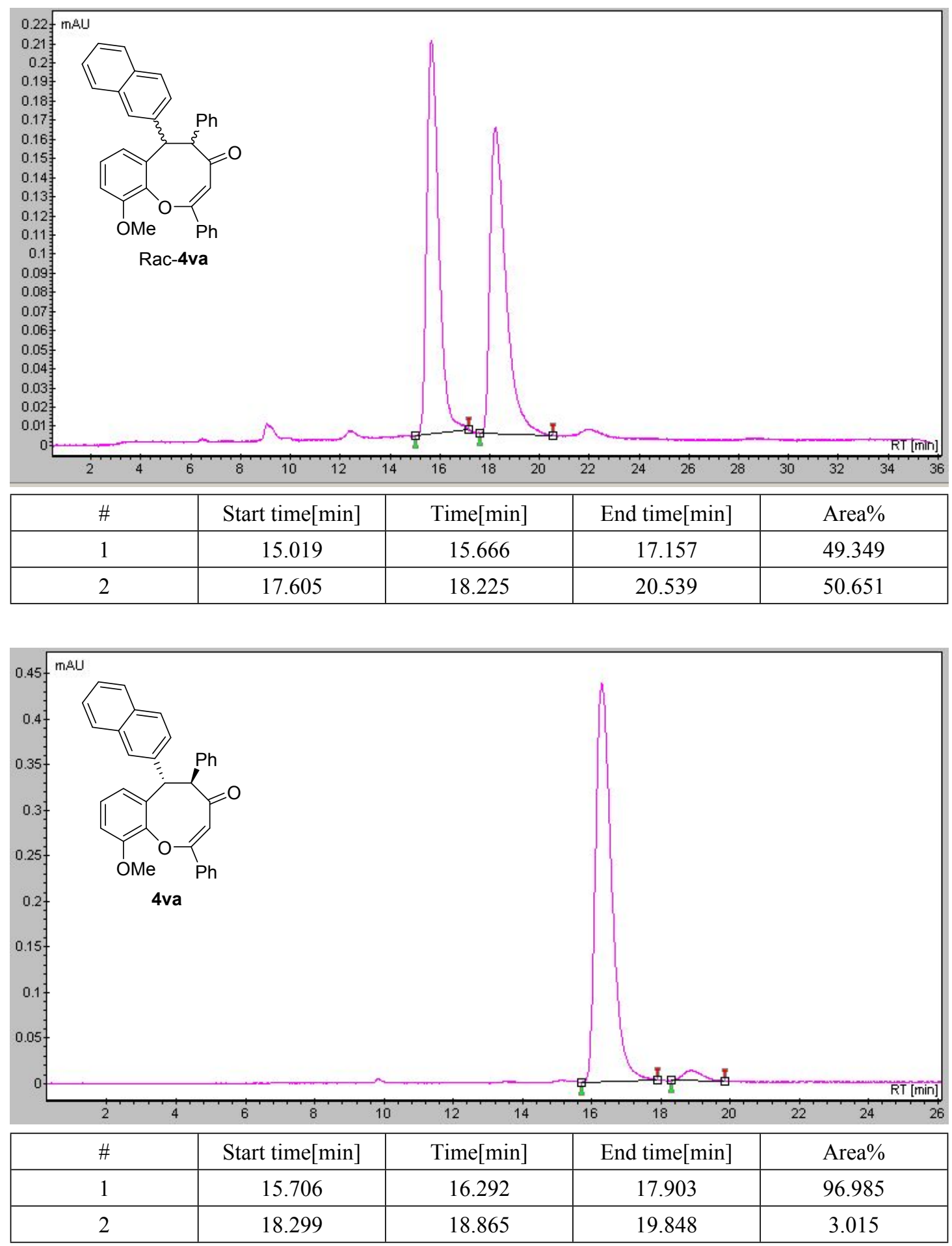

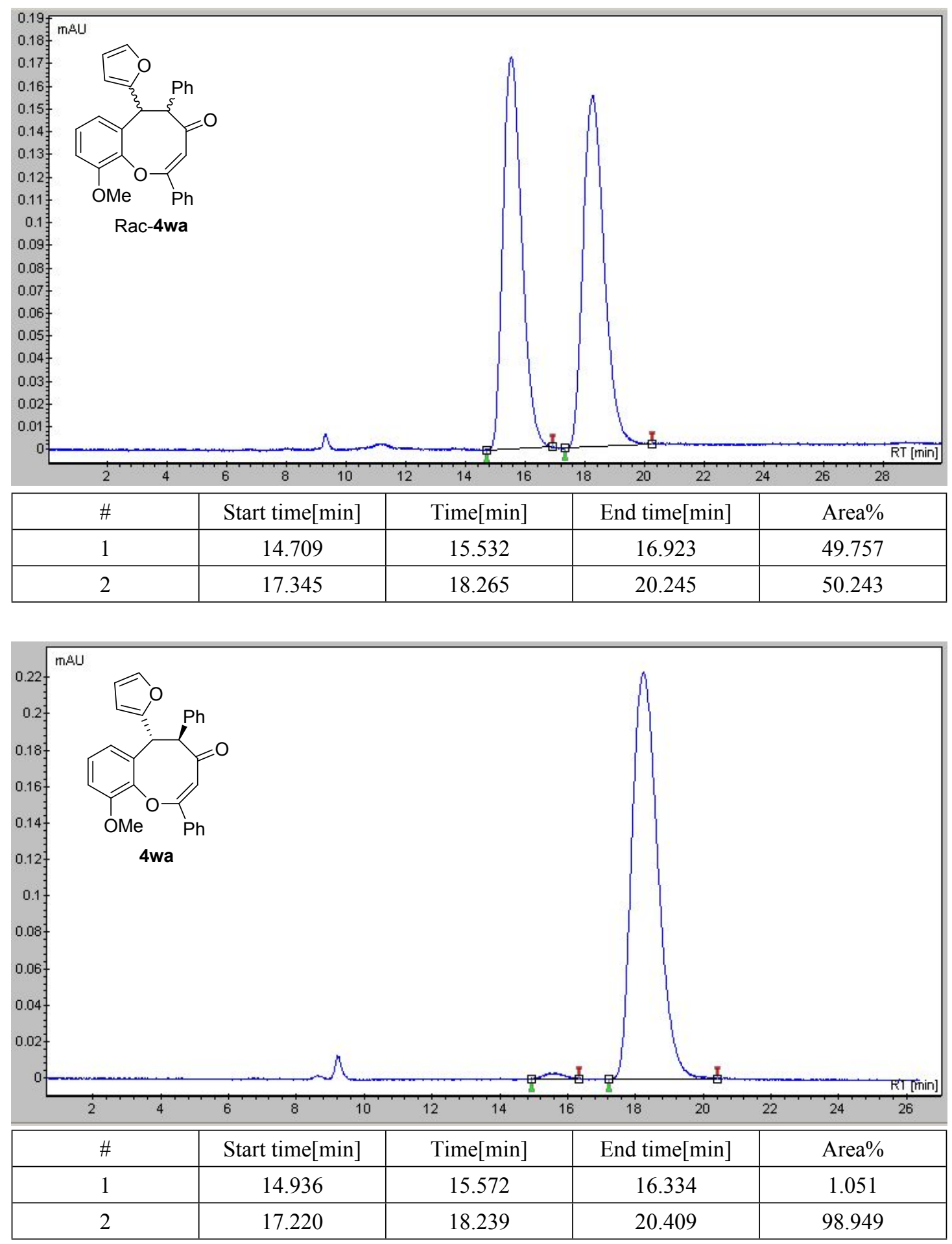

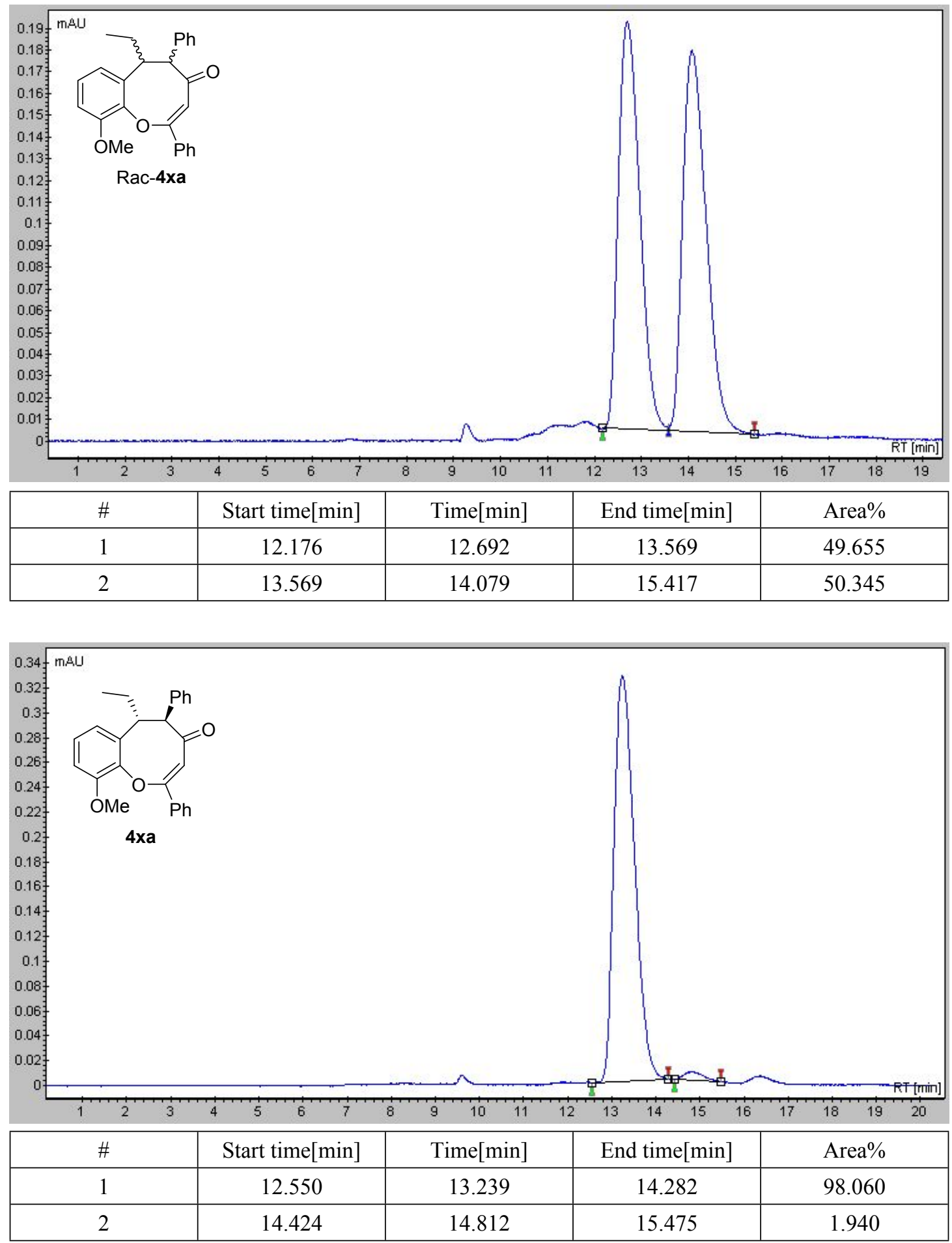

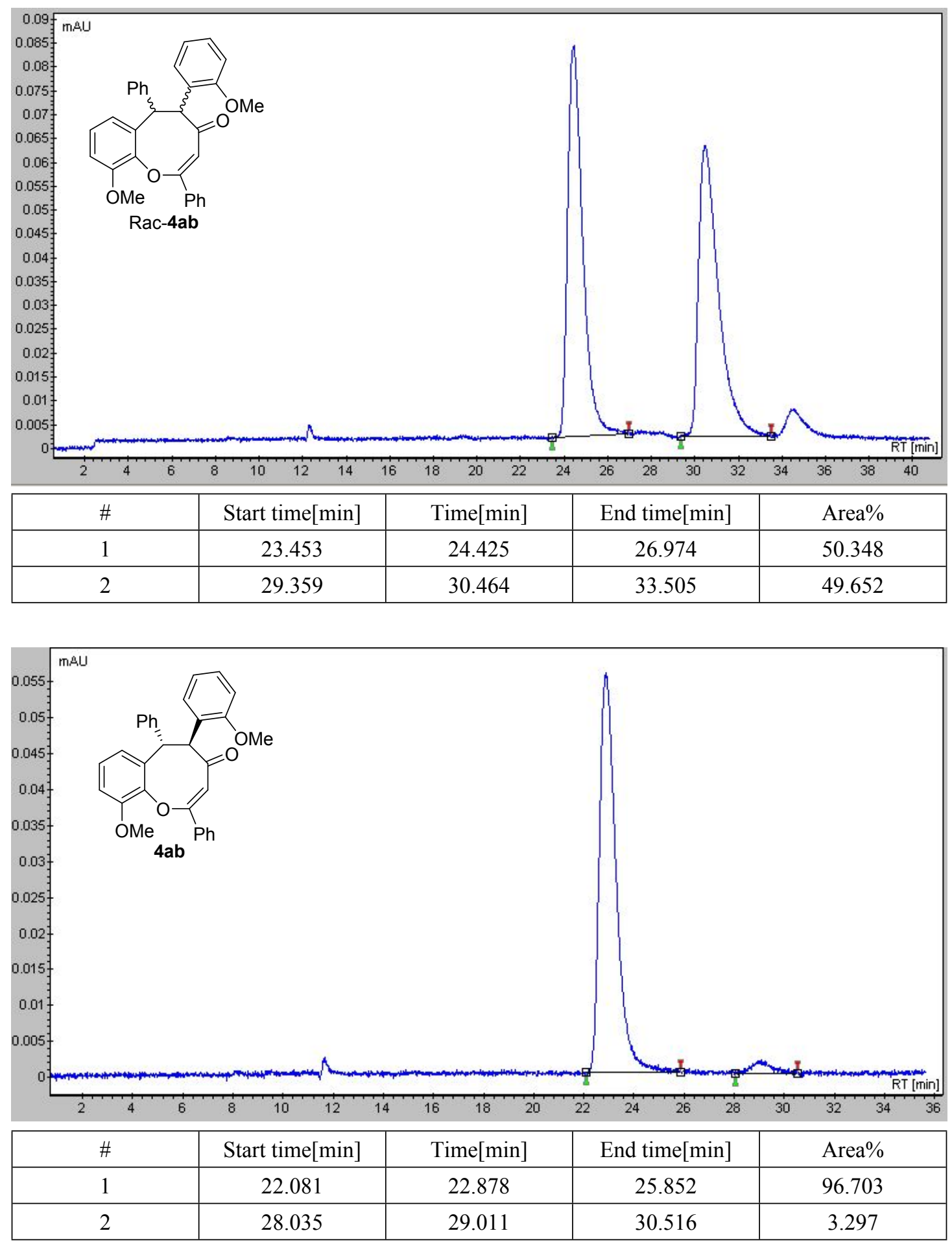

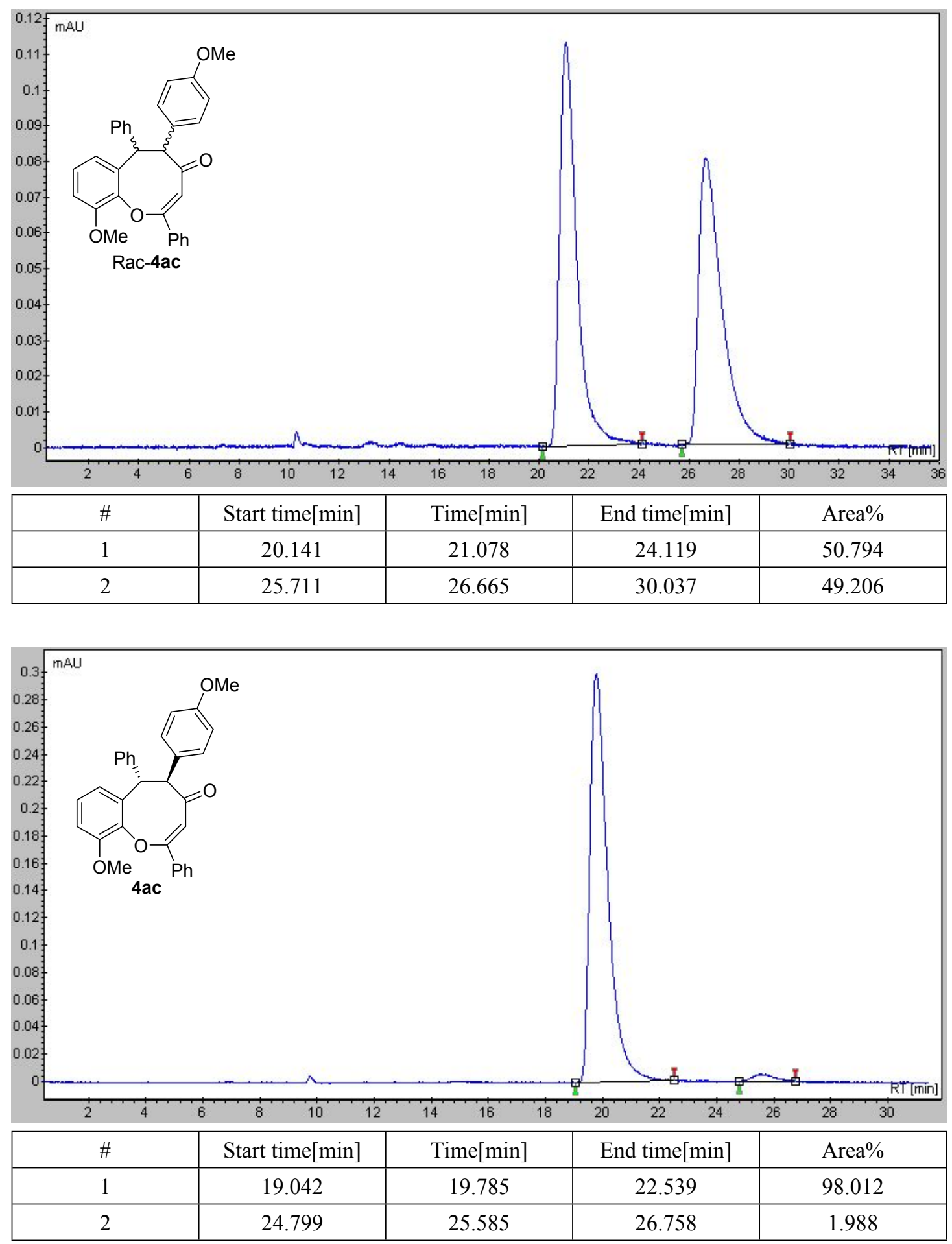

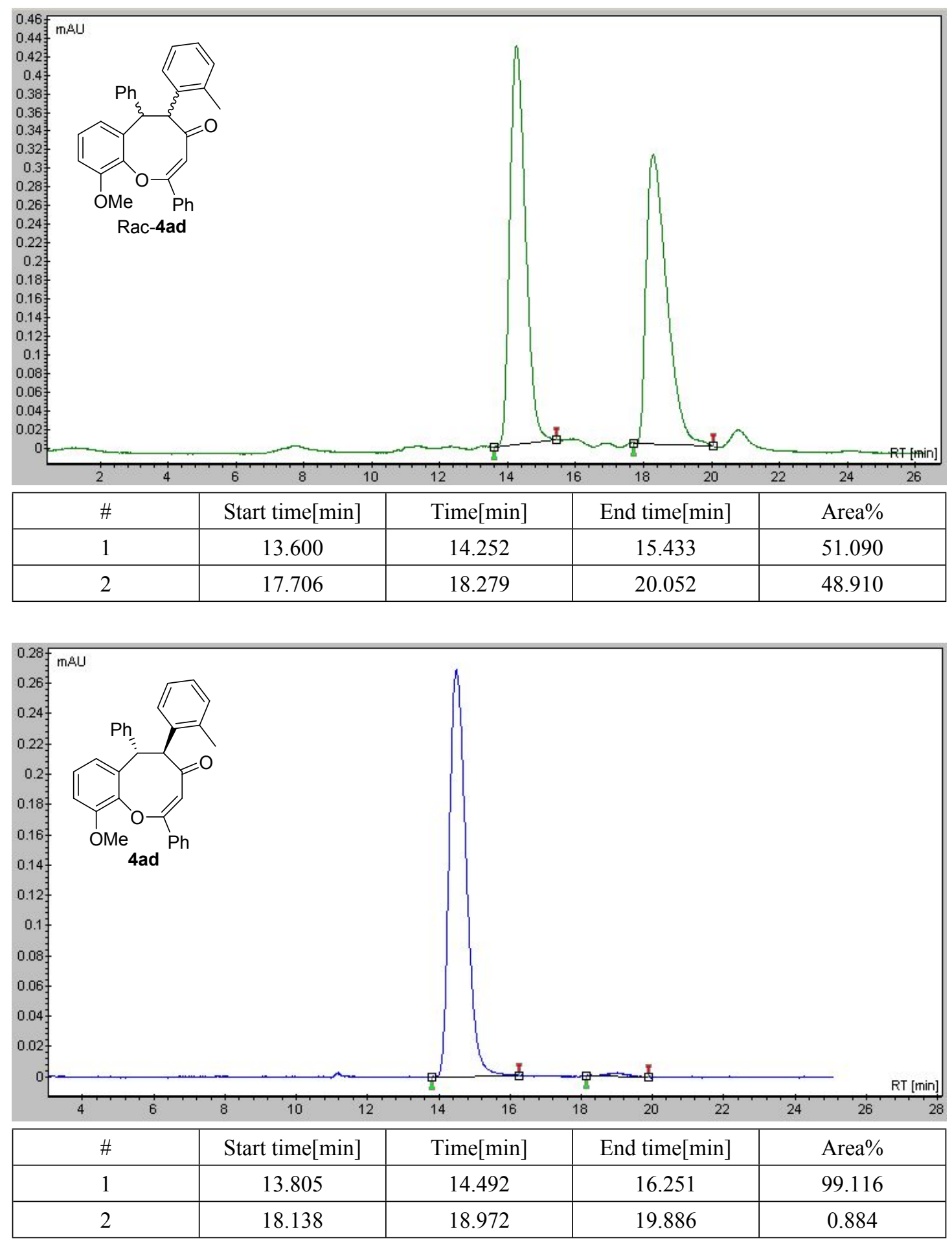

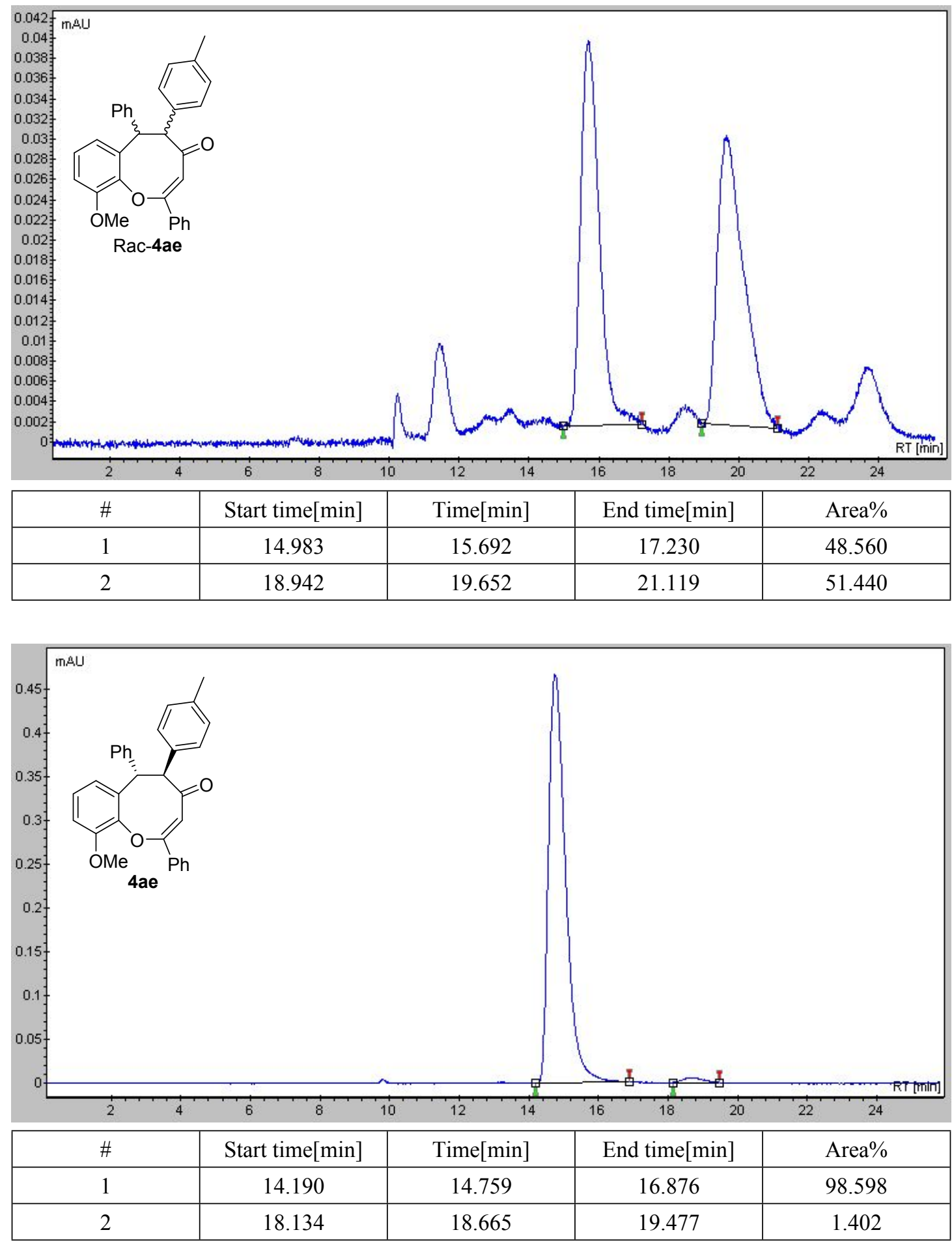

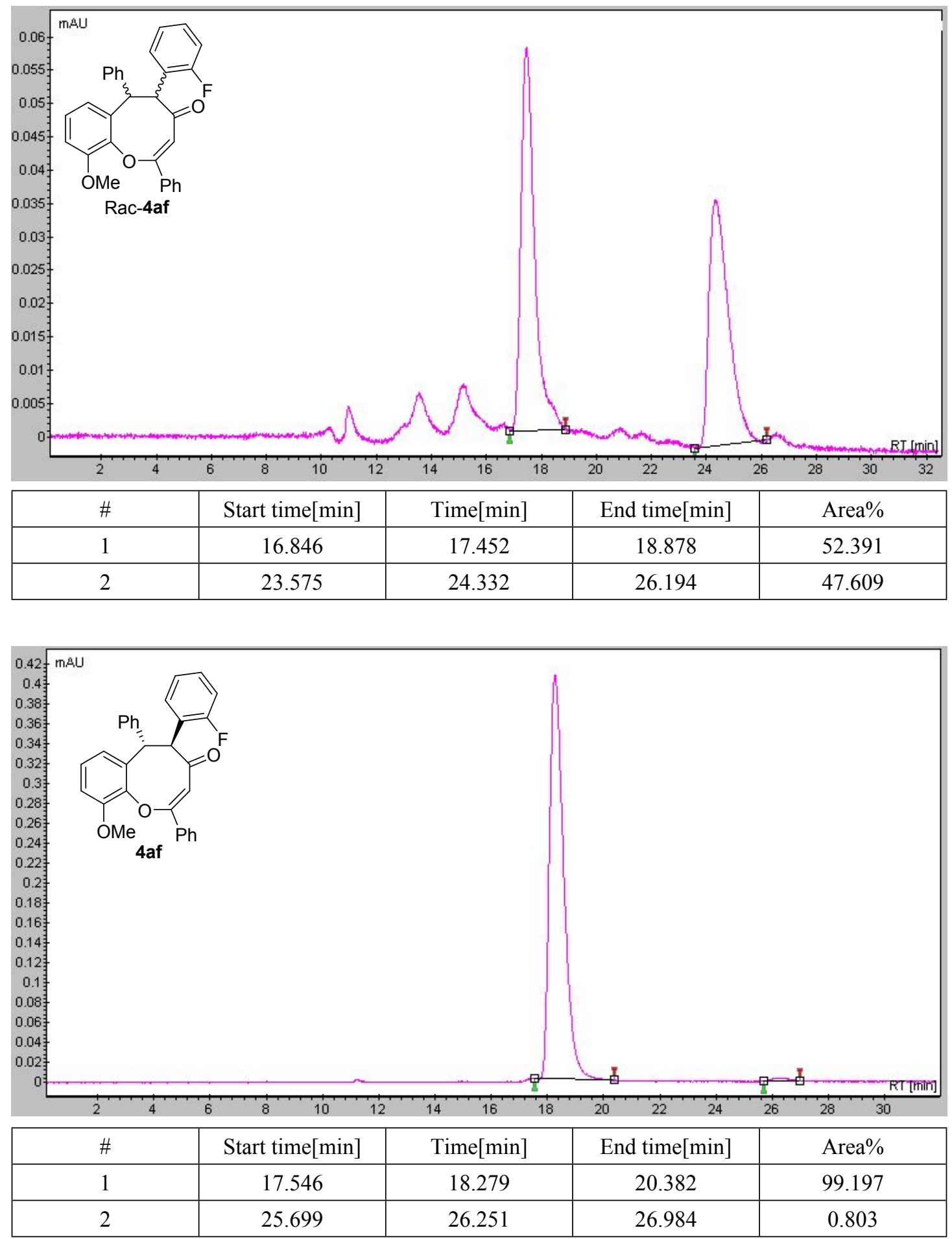

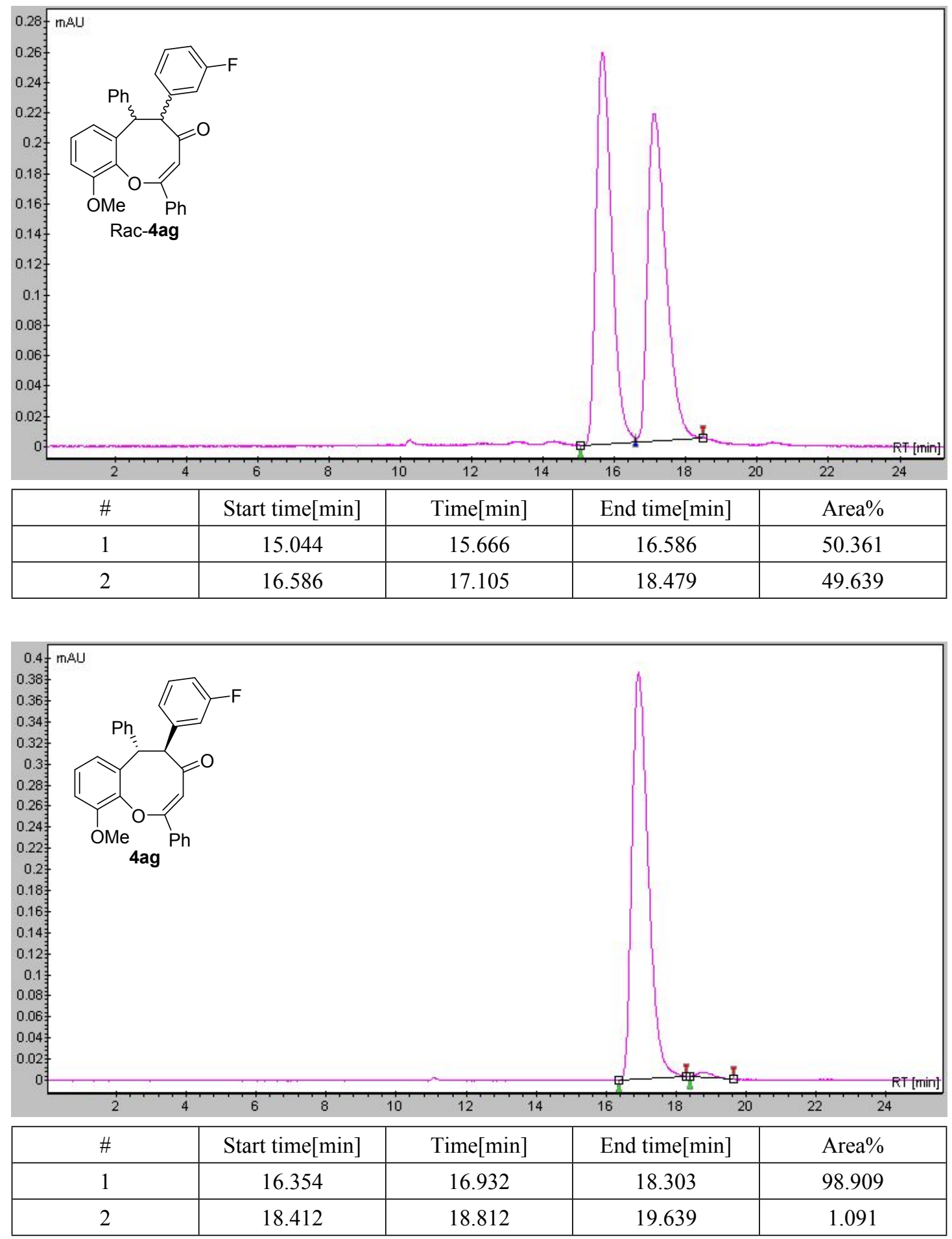

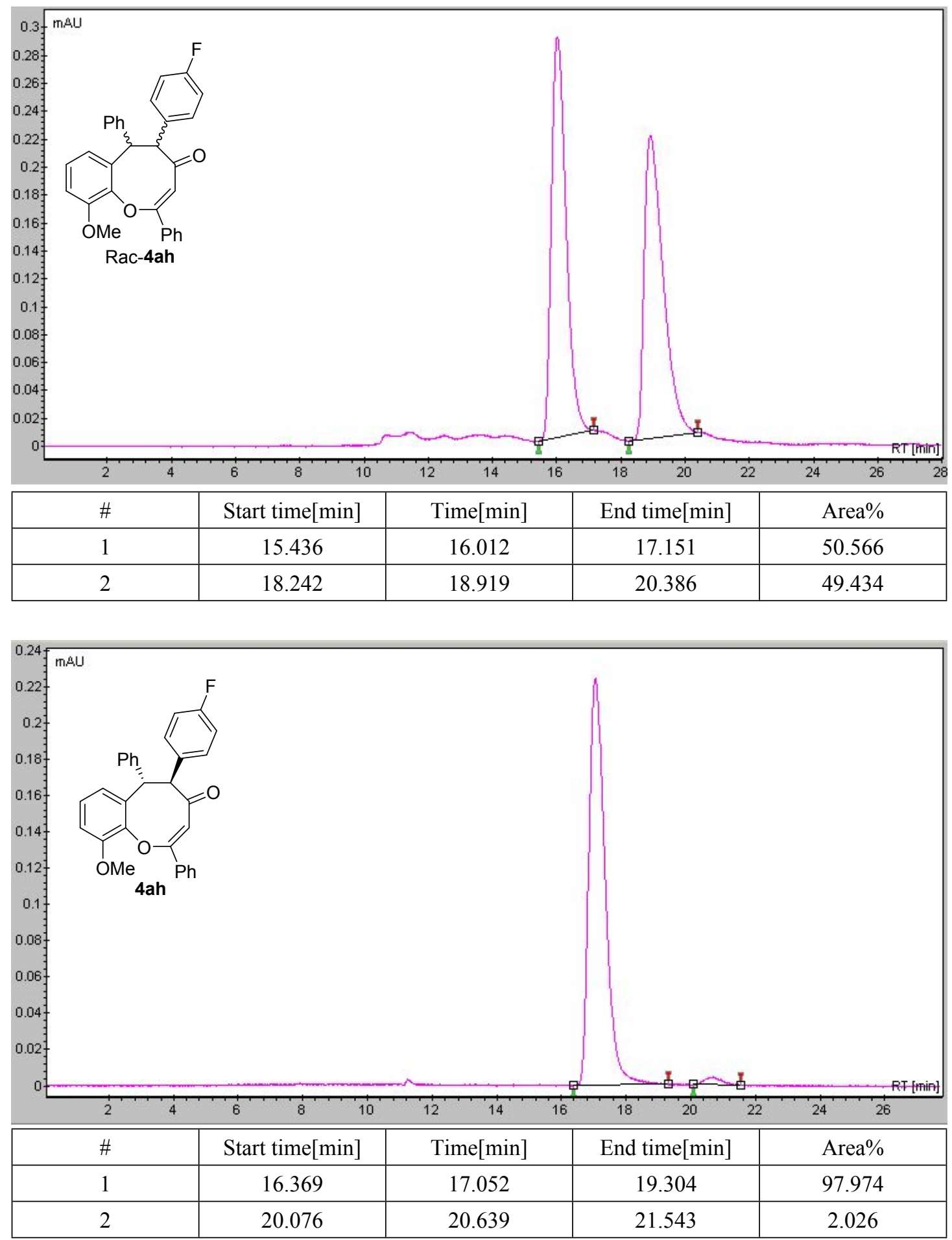

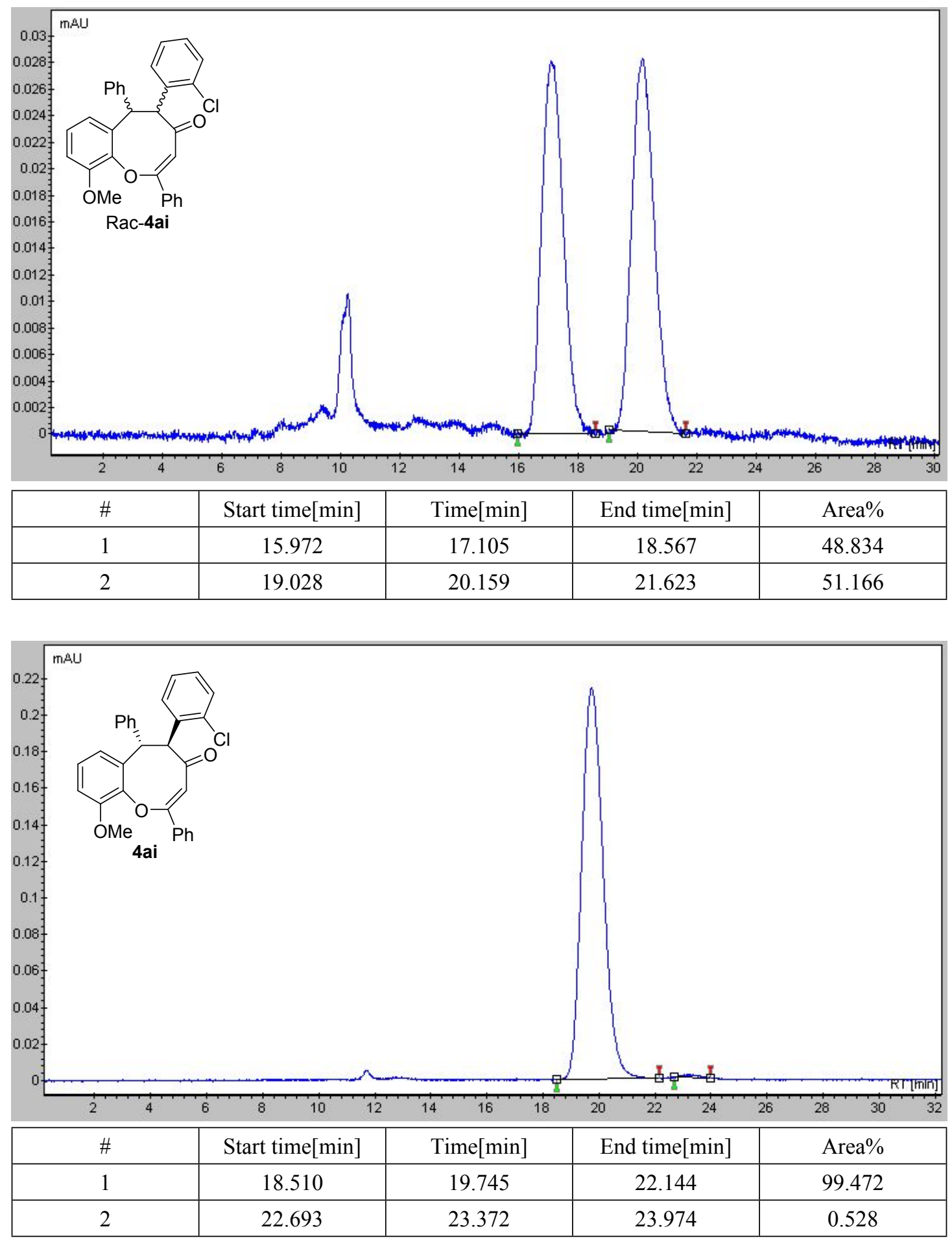

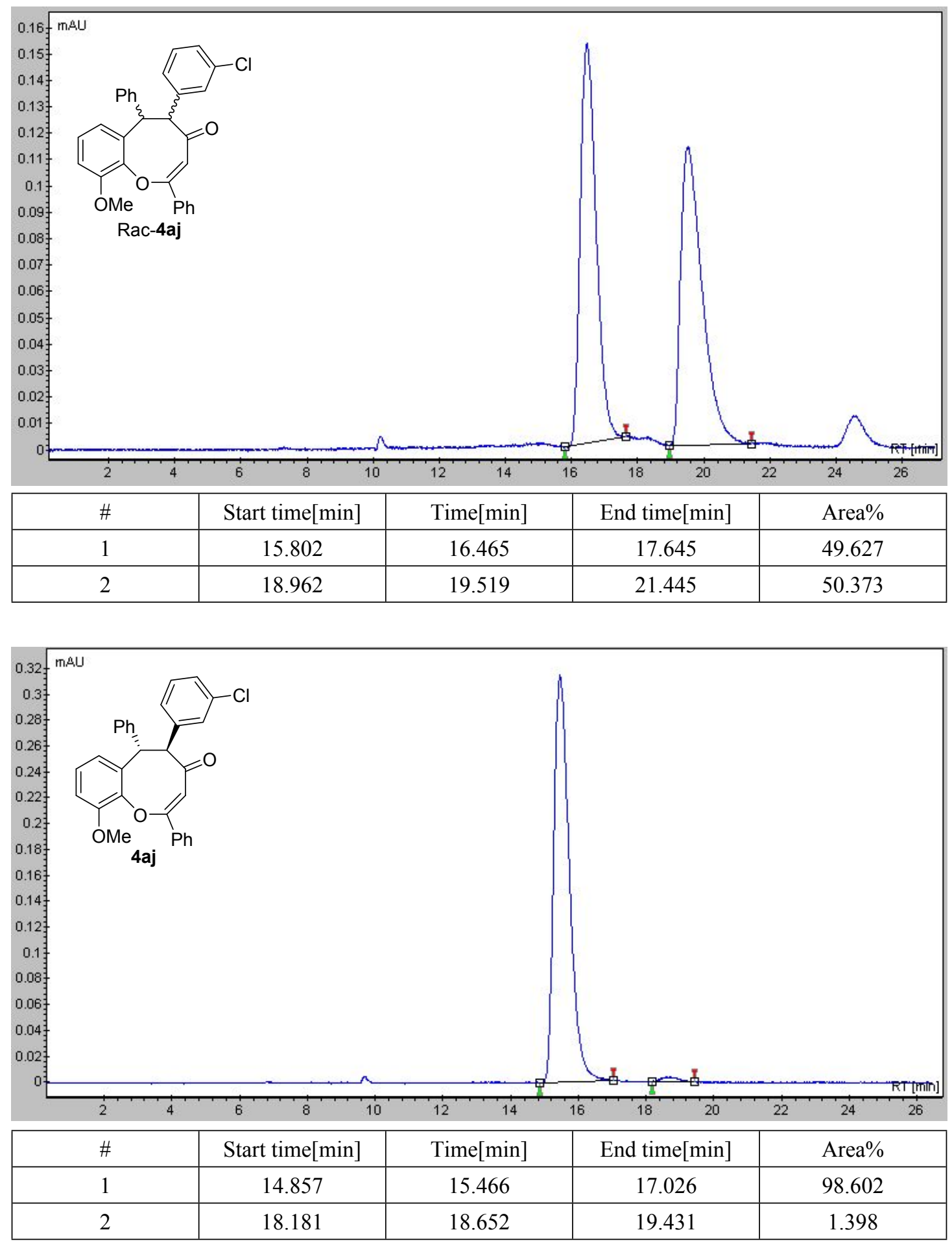

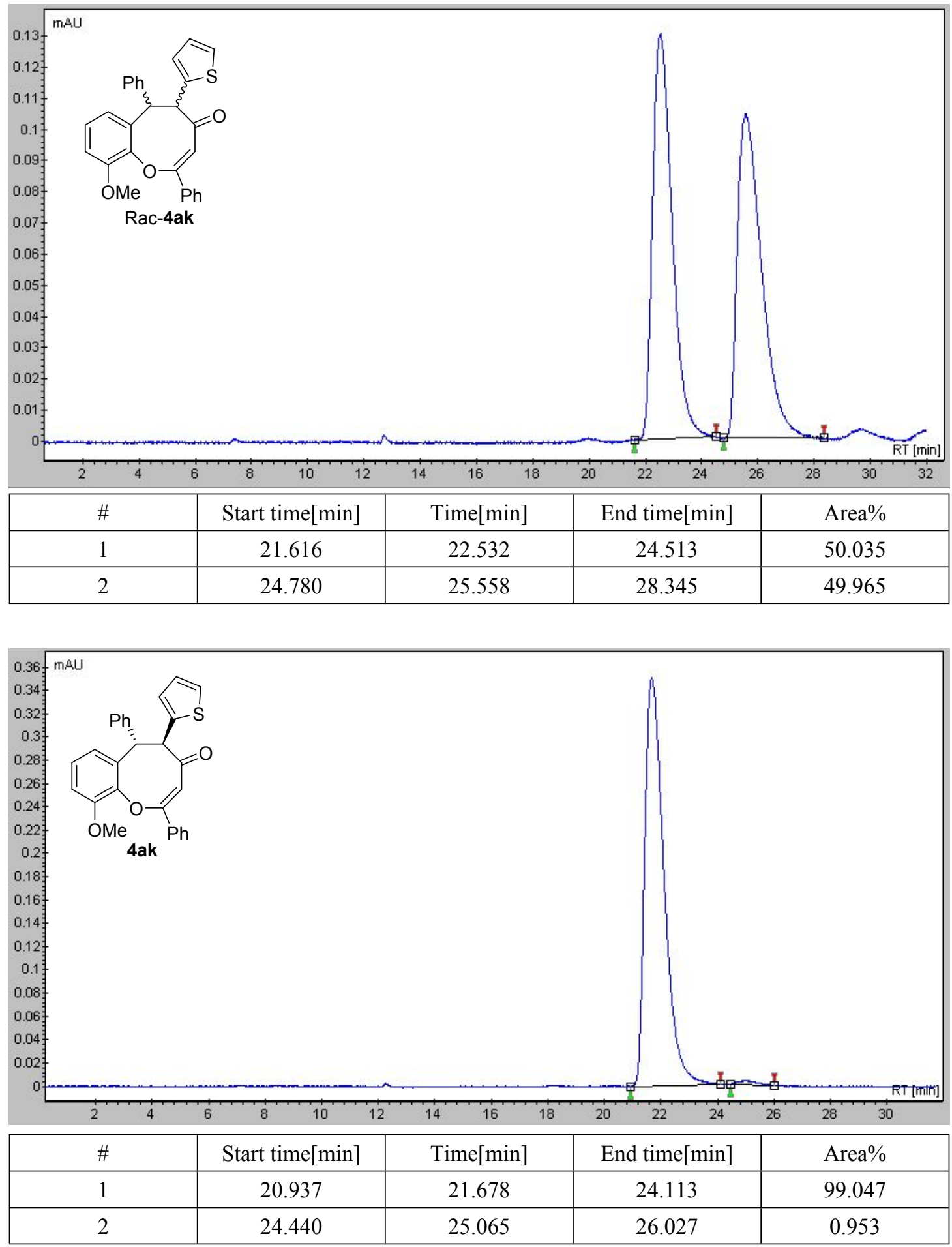

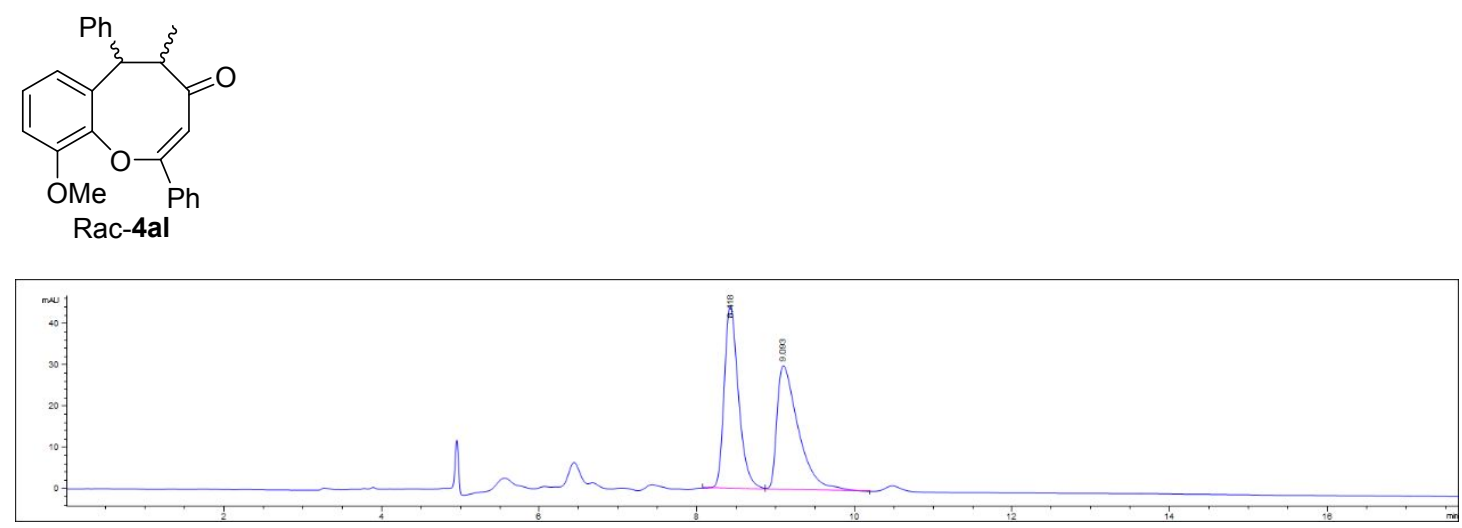

\begin{tabular}{|c|c|c|c|c|c|c|c|}
\hline$\#$ & 时间 & 崔面积 & 㠛高 & 峰赛 & 对称因子 & 峰面积％ & 类型 \\
\hline 1 & 8.418 & 560.2 & 44.4 & 0.193 & 0.688 & 49.561 & BV \\
\hline 2 & 9.093 & 570.1 & 30.1 & 0.2832 & 0.394 & 50.439 & VB \\
\hline
\end{tabular}<smiles>COc1cccc2c1O[C@H](c1ccccc1)C=C([C@H]([18OH])c1ccccc1)C(=O)C2C</smiles>

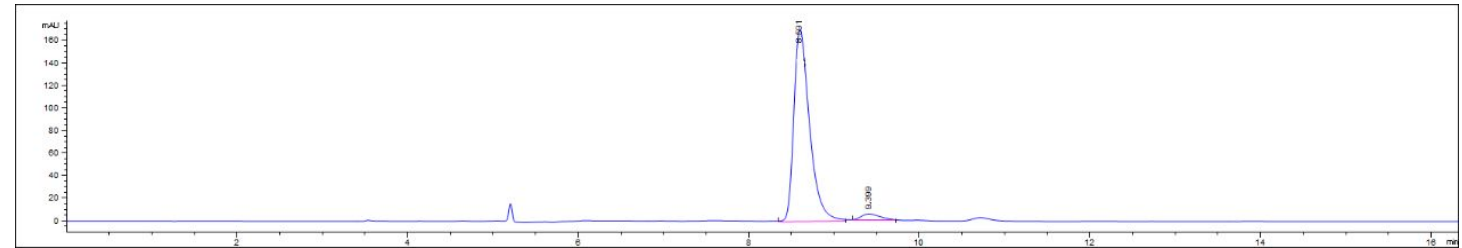

\begin{tabular}{|c|c|c|c|c|c|c|c|}
\hline$\#$ & 时间 & 㠦面积 & 㠎高 & 峰宽 & 对称因子 & 峰面积％ & 类型 \\
\hline 1 & 8.591 & 2195.7 & 170.2 & 0.2151 & 0.619 & 96.242 & MM \\
\hline 2 & 9.399 & 85.7 & 5.5 & 0.2615 & 0.661 & 3.758 & MM \\
\hline
\end{tabular}<smiles>CCCC1C(=O)C=C(c2ccccc2)[C@H](c2ccccc2)Oc2c(O)cccc2[C@H]1c1ccccc1</smiles>

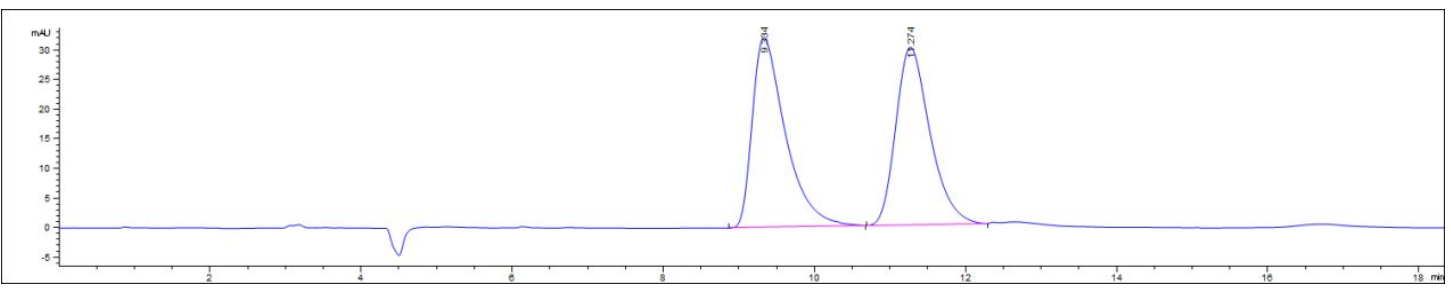

\begin{tabular}{|c|c|c|c|c|c|c|c|}
\multicolumn{1}{|c}{ \# } & \multicolumn{1}{c}{ 时间 } & \multicolumn{1}{c}{ 峰面积 } & \multicolumn{1}{c|}{ 崔高 } & \multicolumn{2}{c|}{ 峰宽 } & \multicolumn{2}{c|}{ 对称因子 峰面积 \% 类型 } \\
\hline 1 & 9.334 & 971.9 & 31.9 & 0.4577 & 0.504 & 50.921 & BB \\
\hline 2 & 11.274 & 936.8 & 29.8 & 0.4784 & 0.705 & 49.079 & BB \\
\hline
\end{tabular}



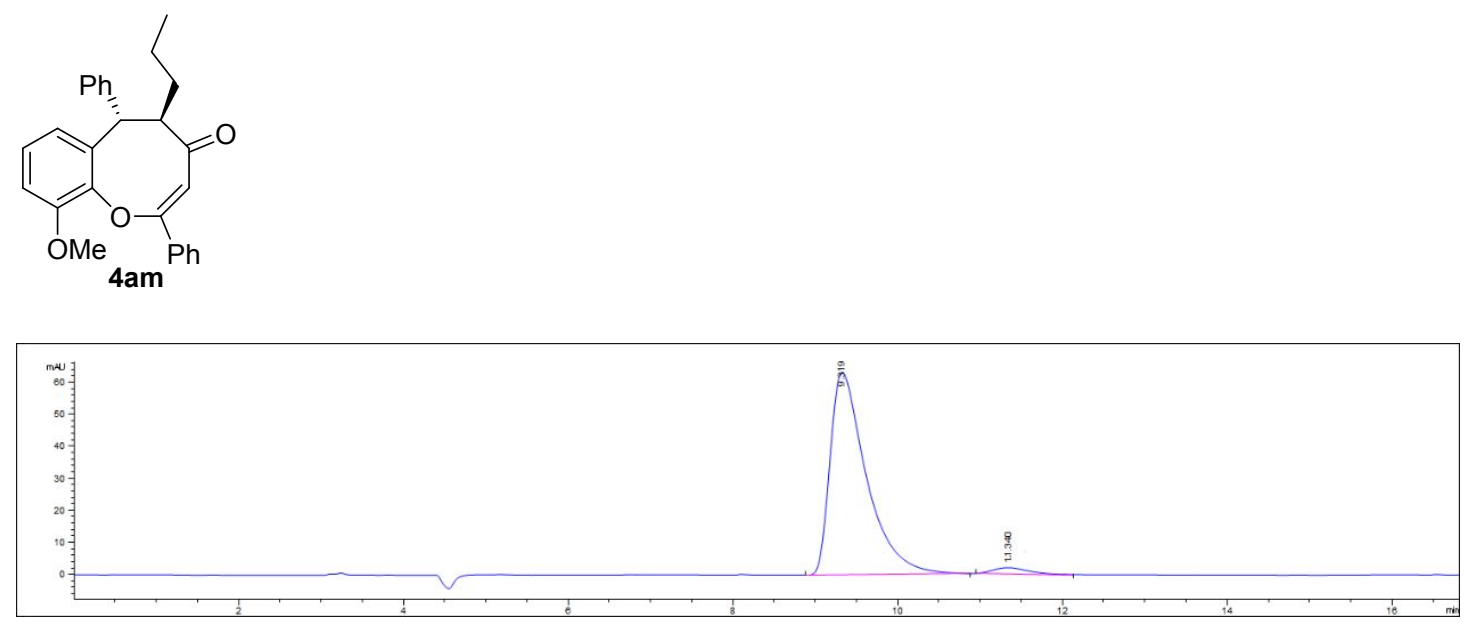

\begin{tabular}{|c|c|c|c|c|c|c|c|}
\hline$\#$ & 时间 & 㠦面积 & 崔高 & 峰宽 & 对称因子 & 峰面积 \% & 类琹 \\
\hline 1 & 9.319 & 1927.5 & 63.1 & 0.4544 & 0.465 & 96.495 & BB \\
\hline 2 & 11.34 & 70 & 2.1 & 0.5659 & 0.559 & 3.505 & $\mathrm{MM}$ \\
\hline
\end{tabular}<smiles>COc1cccc2c1OC(c1ccccc1)CC(=O)C(Cc1ccccc1)C2c1ccccc1</smiles>

Rac-4an

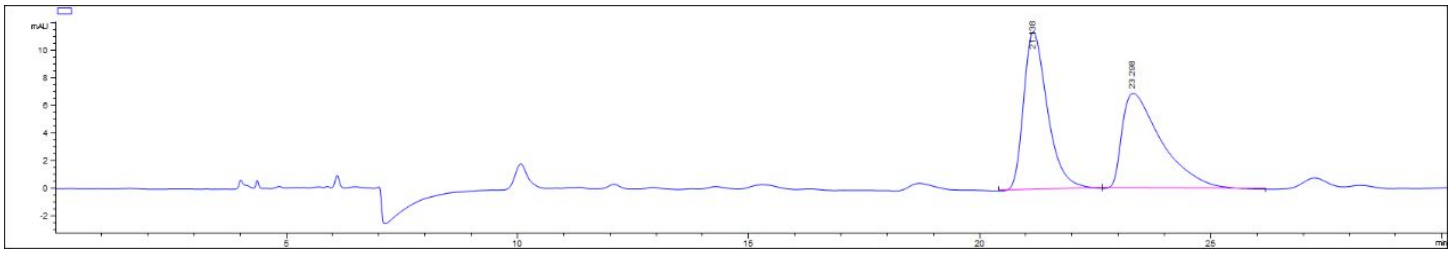

\begin{tabular}{|c|c|c|c|c|c|c|c|}
\hline$\#$ & 时间 & 崔面积 & 峰高 & 峰宽 & 对称因子 & 峰面积％ & 类型 \\
\hline 1 & 21.138 & 414.5 & 11.5 & 0.5498 & 0.638 & 50.009 & $\mathrm{BB}$ \\
\hline 2 & 23.298 & 414.4 & 6.9 & 0.8524 & 0.359 & 49.991 & $\mathrm{BB}$ \\
\hline
\end{tabular}

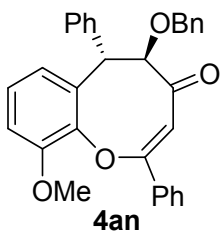

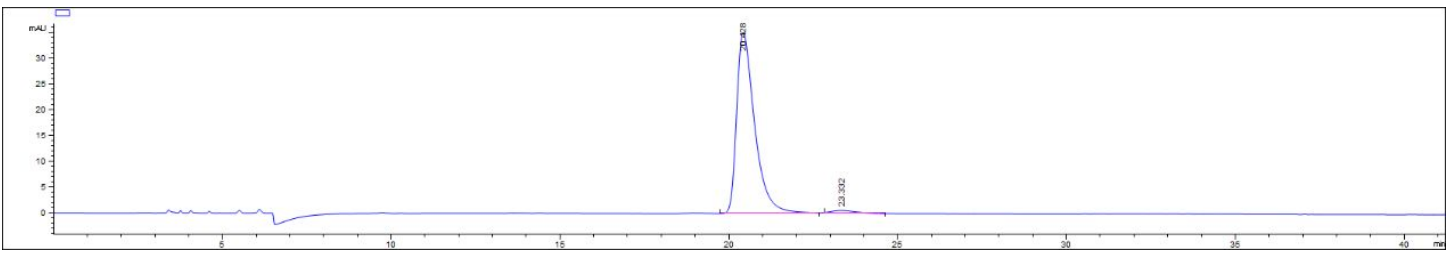

\begin{tabular}{|c|c|c|c|c|c|c|c|}
\multicolumn{1}{c}{ \# } & \multicolumn{1}{c}{ 时间 } & \multicolumn{1}{c}{ 峰面积 } & \multicolumn{1}{c}{ 峰高 } & \multicolumn{2}{c|}{ 峰宽 } & \multicolumn{2}{c|}{ 对称因子 峰面积 \% 类型 } \\
\hline 1 & 20.428 & 1290.7 & 35 & 0.5559 & 0.538 & 97.828 & BB \\
\hline 2 & 23.332 & 28.7 & $5.8 \mathrm{E}-1$ & 0.8237 & 0.709 & 2.172 & MM \\
\hline
\end{tabular}




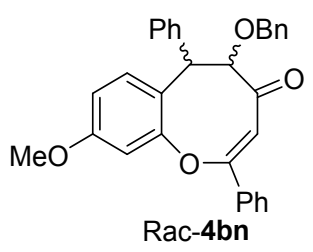

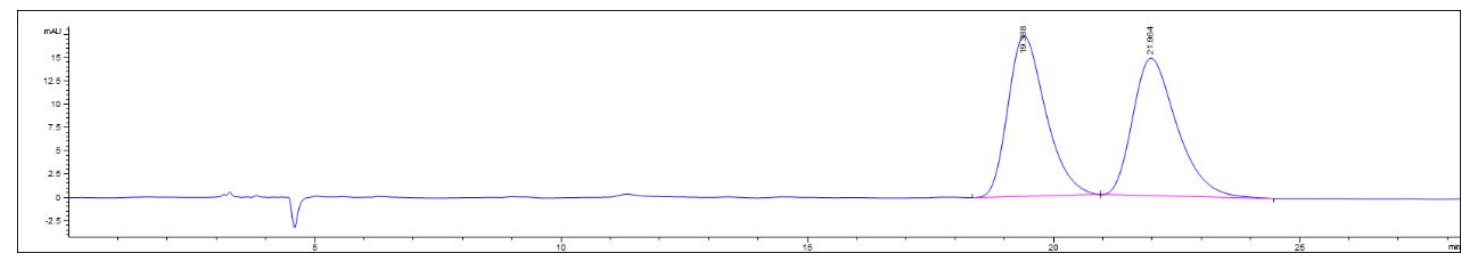

\begin{tabular}{|c|c|c|c|c|c|c|c|}
\hline$\#$ & 时间 & 峰面积 & 峰高 & 崔宽 & 对称因子 & 峰面积 \% & 类型 \\
\hline 1 & 19.388 & 917.7 & 17.2 & 0.8127 & 0.707 & 50.001 & BB \\
\hline 2 & 21.964 & 917.7 & 14.7 & 0.9464 & 0.666 & 49.999 & $\mathrm{BB}$ \\
\hline
\end{tabular}<smiles>COc1ccc2c(c1)OC(c1ccccc1)C(c1ccccc1)C(=O)C=C2C(=O)c1ccccc1</smiles>

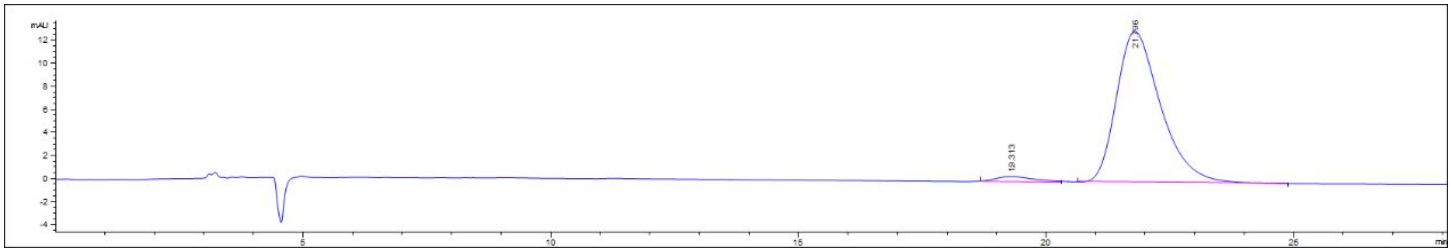

\begin{tabular}{|c|c|c|c|c|c|c|c|}
\hline$\#$ & 时间 & 峰面积 & 崔高 & 峰宽 & 对称因子 & 峰面积 \% & 类型 \\
\hline 1 & 19.313 & 25.9 & $4.6 \mathrm{E}-1$ & 0.9367 & 0.638 & 3.075 & MM \\
\hline 2 & 21.796 & 817.1 & 13.2 & 0.9283 & 0.672 & 96.925 & BB \\
\hline
\end{tabular}<smiles>O=C1C=C(c2ccccc2)[C@H]([OH2+])[C@H](c2ccccc2)Oc2c(F)cccc2[C@H]1c1ccccc1</smiles>

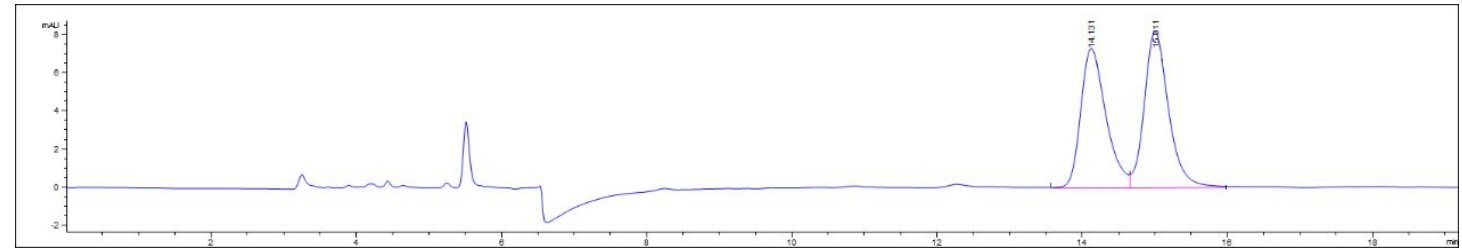

\begin{tabular}{|c|c|c|c|c|c|c|c|} 
\# & \multicolumn{1}{c}{ 时间 } & \multicolumn{1}{c}{ 峰面积 } & \multicolumn{1}{c|}{ 峰高 } & \multicolumn{3}{c|}{ 峰宽 } & \multicolumn{2}{c|}{ 对称因子 峰面积 \% 类型 } \\
\hline 1 & 14.131 & 180.7 & 7.4 & 0.4075 & 0.702 & 48.192 & MF \\
\hline 2 & 15.011 & 194.2 & 8.3 & 0.3917 & 0.762 & 51.808 & FM \\
\hline
\end{tabular}<smiles>[O]C1c2cccc(F)c2OC(=O)/C=C(\c2ccccc2)C([OH2+])C1c1ccccc1</smiles> 


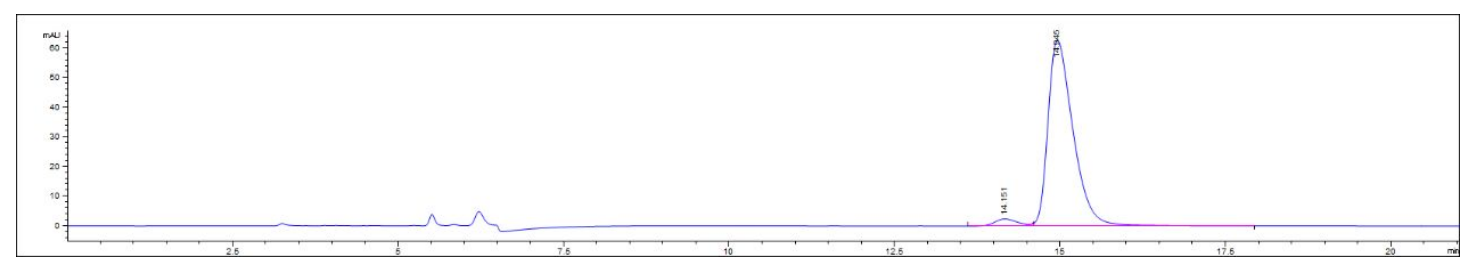

\begin{tabular}{|c|c|c|c|c|c|c|c|}
\hline$\#$ & 时间 & 峰面积 & 崔高 & 峰宽 & 对称因子 & 峰面积 \% & 类型 \\
\hline 1 & 14.151 & 56.2 & 2.4 & 0.3603 & 0.777 & 3.290 & BVE \\
\hline 2 & 14.945 & 1651 & 62.7 & 0.3991 & 0.662 & 96.710 & VB R \\
\hline
\end{tabular}

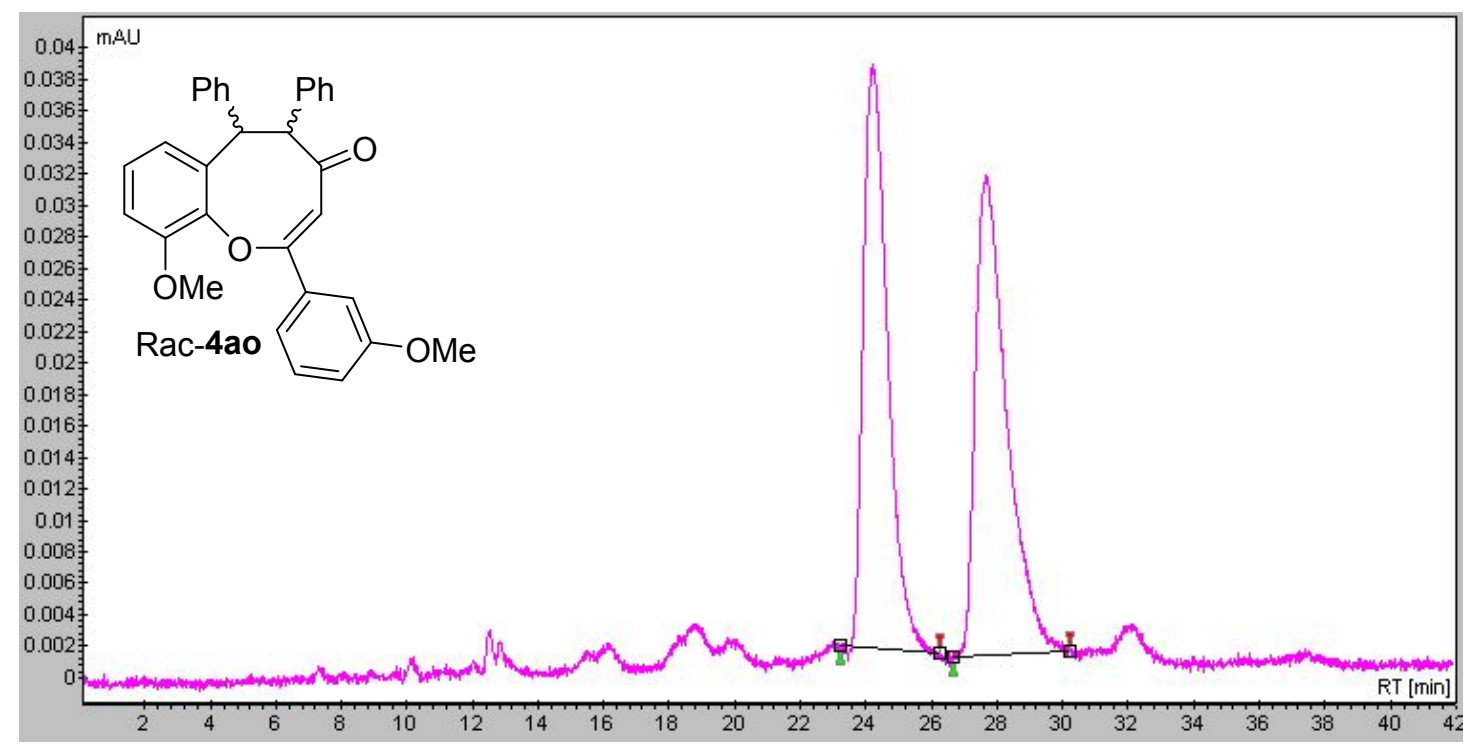

\begin{tabular}{|c|c|c|c|c|}
\hline$\#$ & Start time[min] & Time[min] & End time[min] & Area\% \\
\hline 1 & 23.222 & 24.225 & 26.256 & 48.720 \\
\hline 2 & 26.665 & 27.651 & 30.224 & 51.280 \\
\hline
\end{tabular}

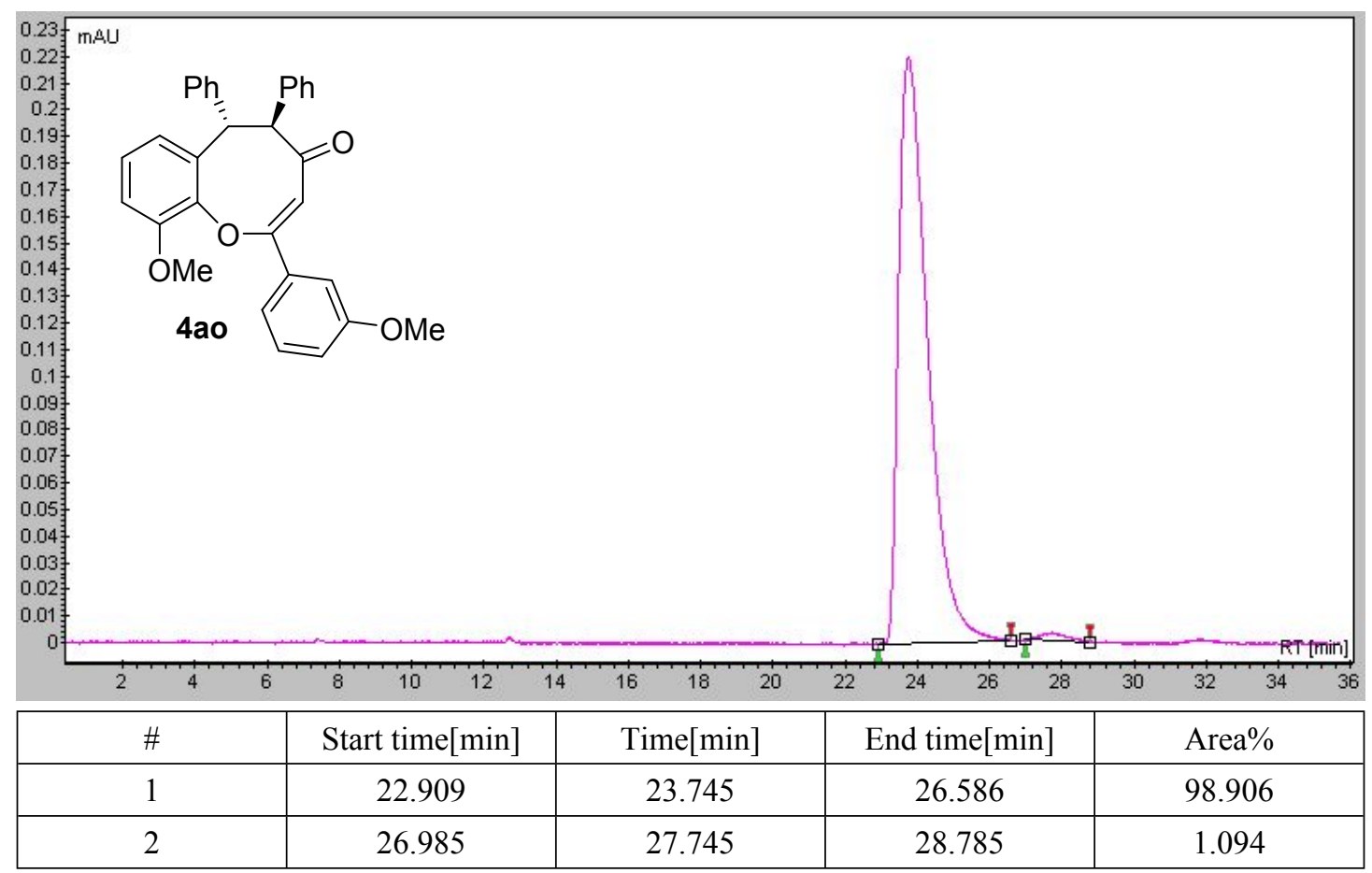



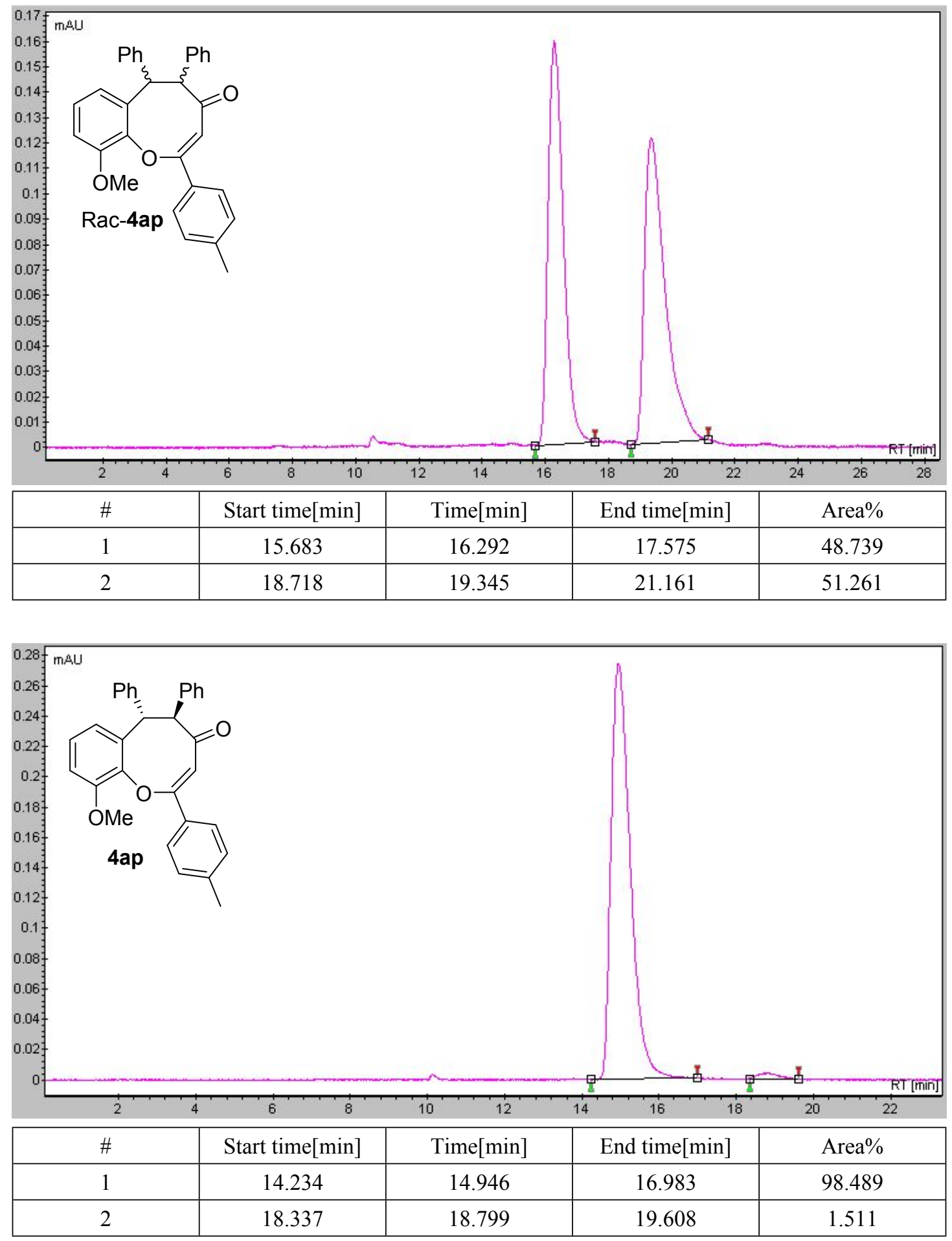

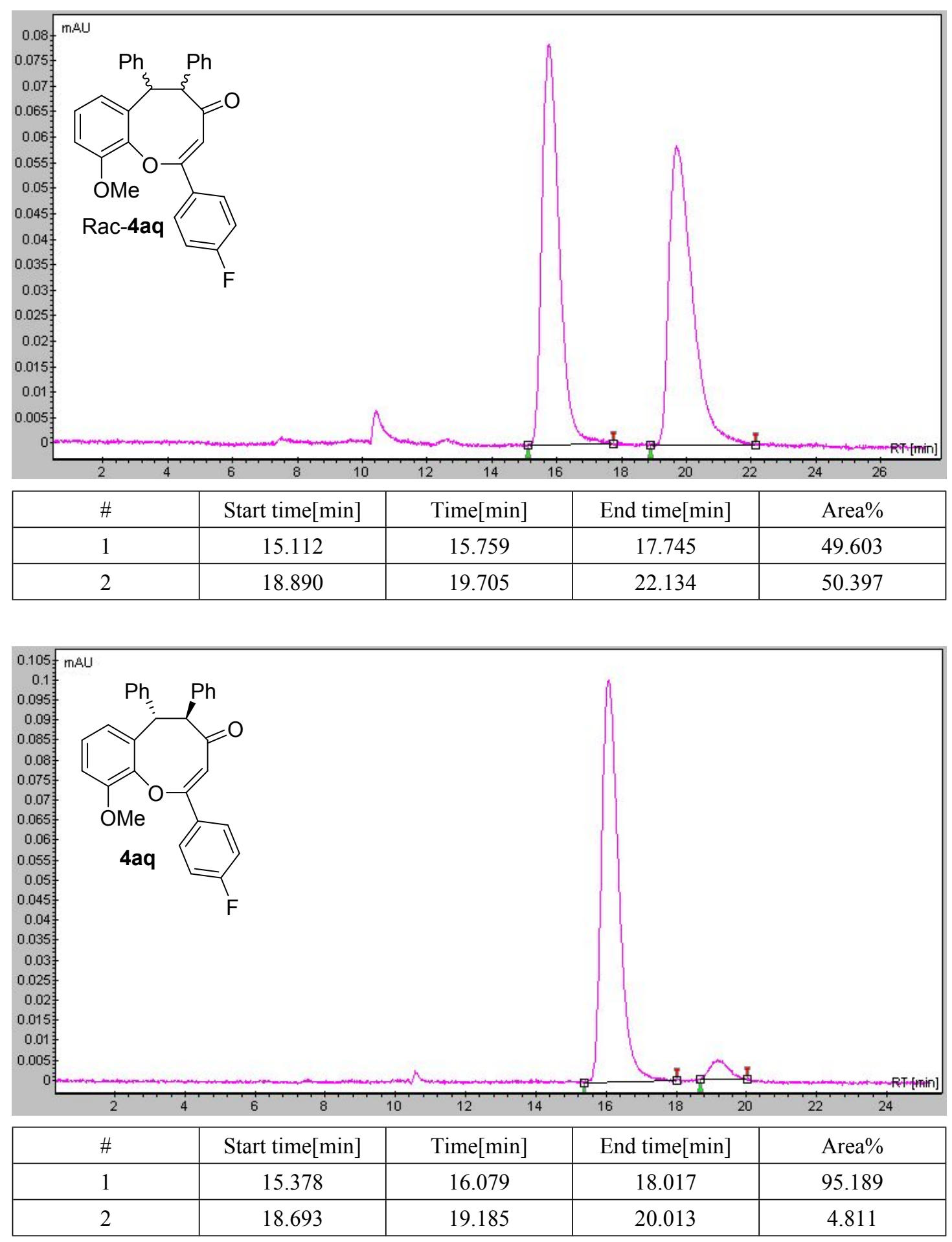

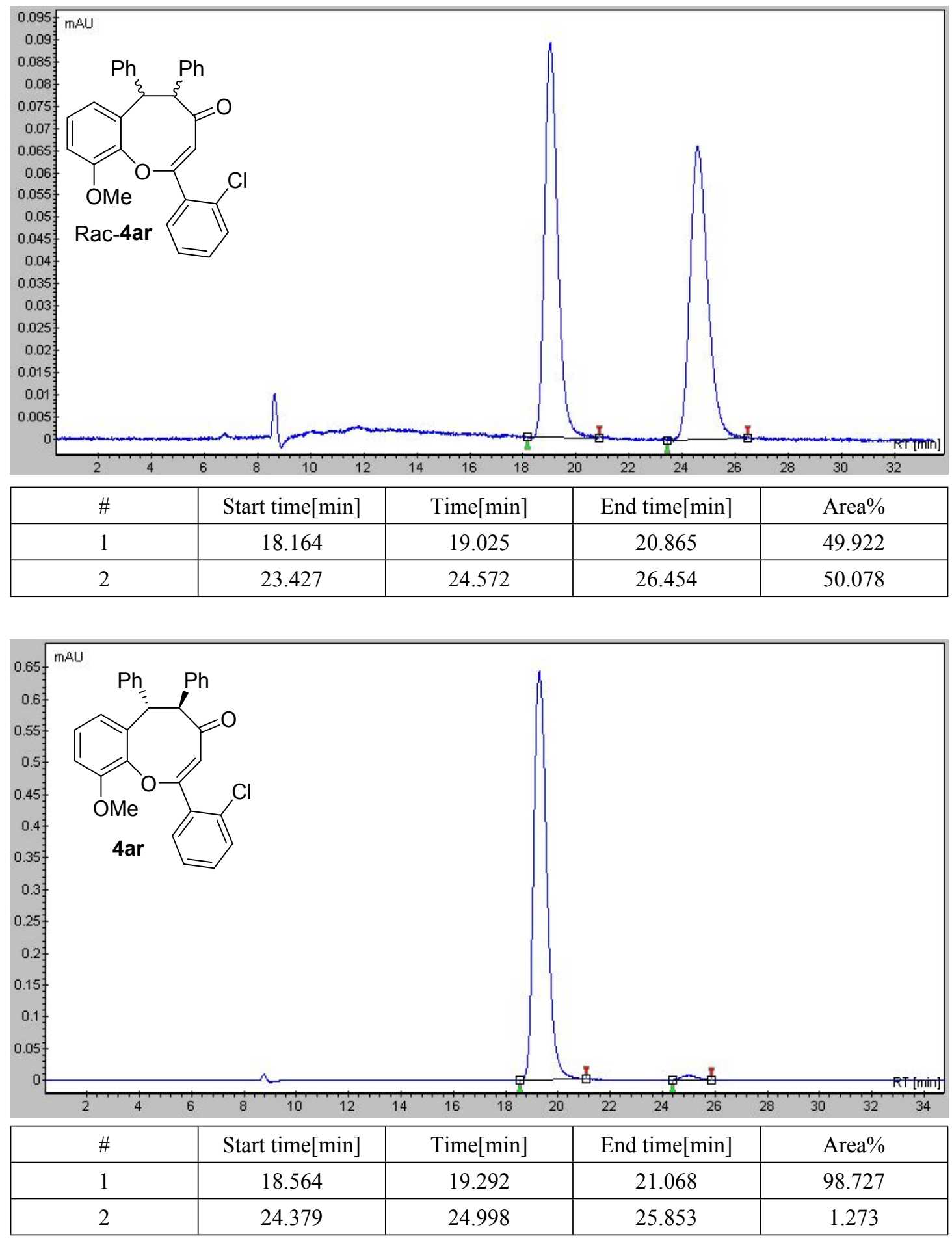

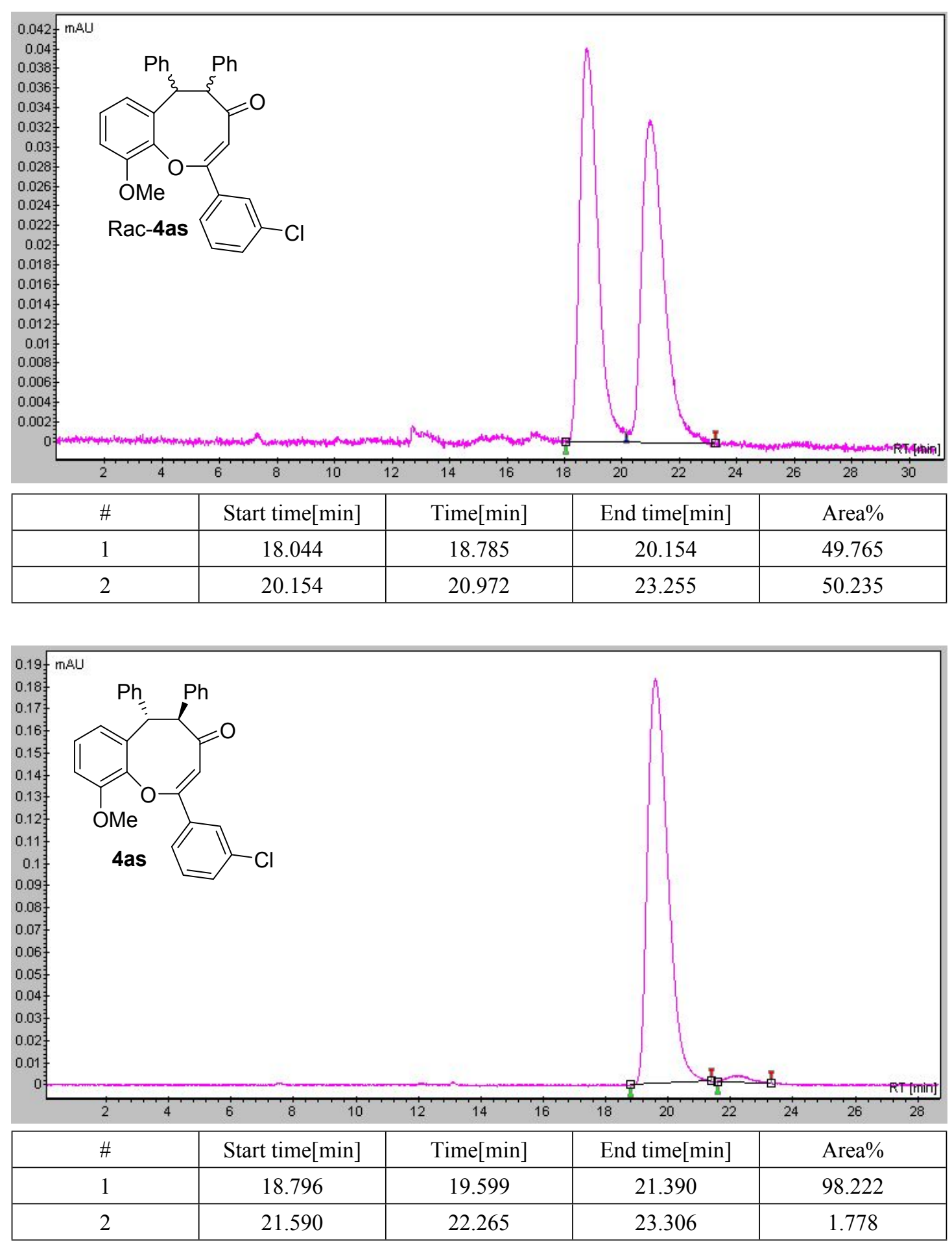

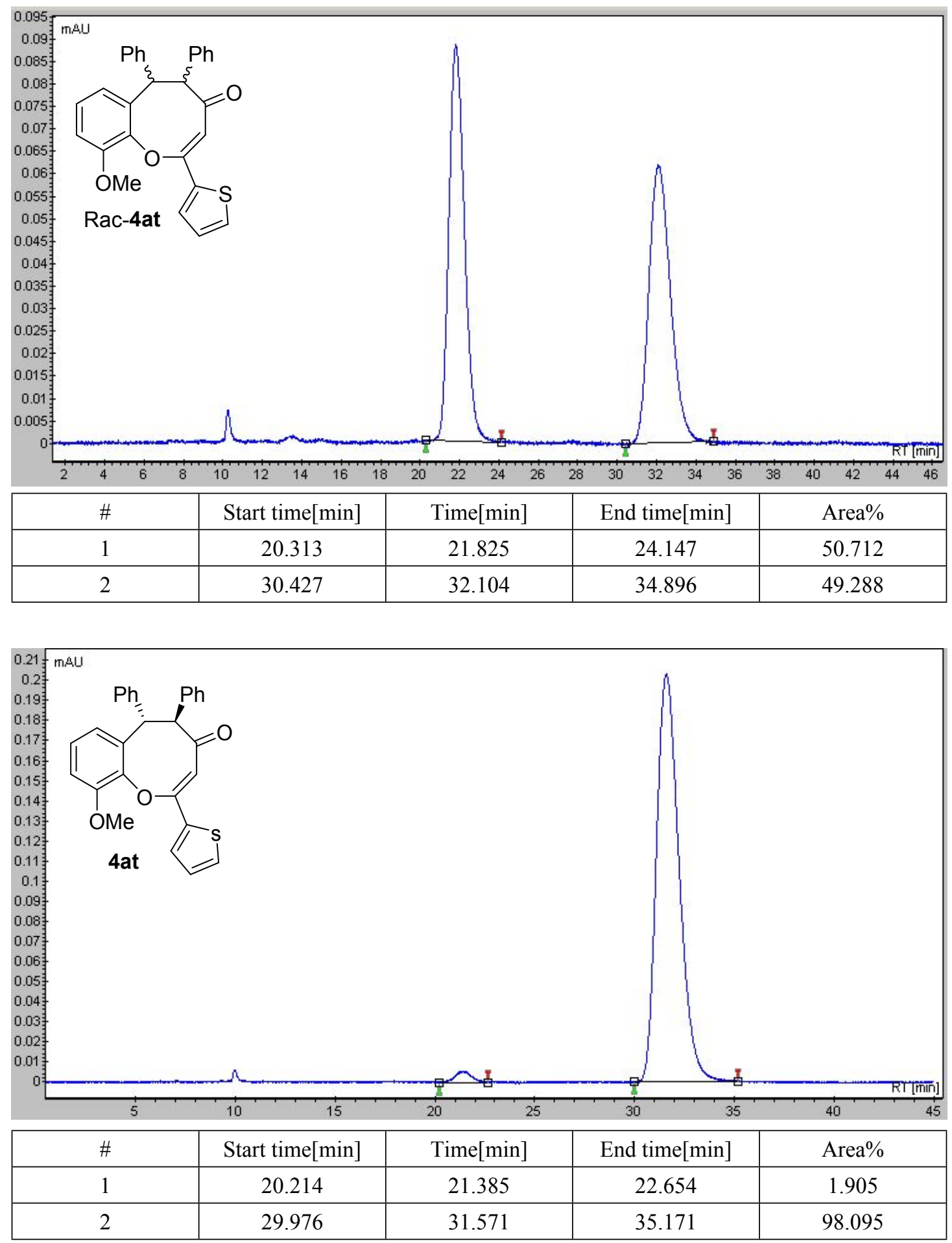

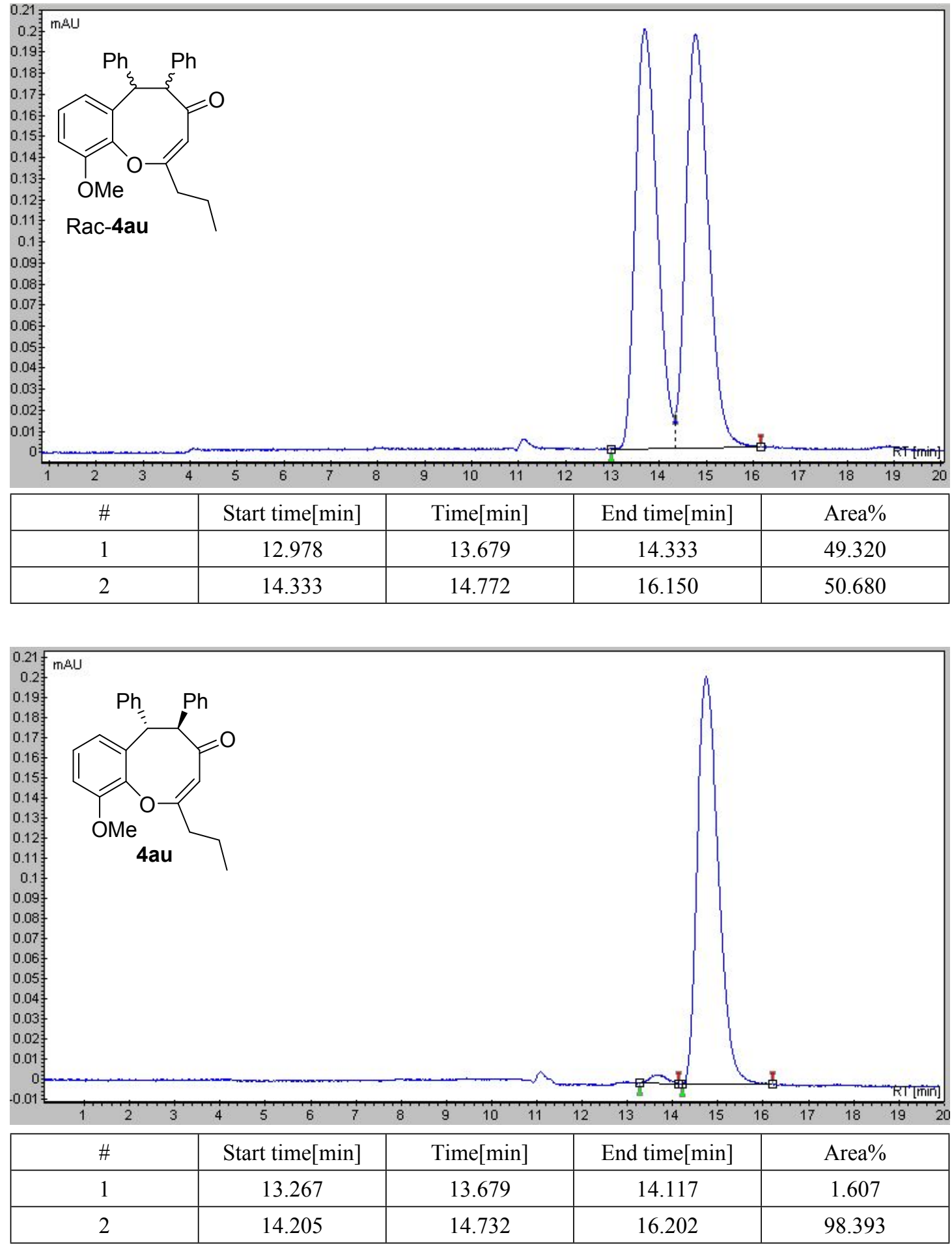<smiles>[R4]C(=O)N[14C](C)(C)C</smiles> 


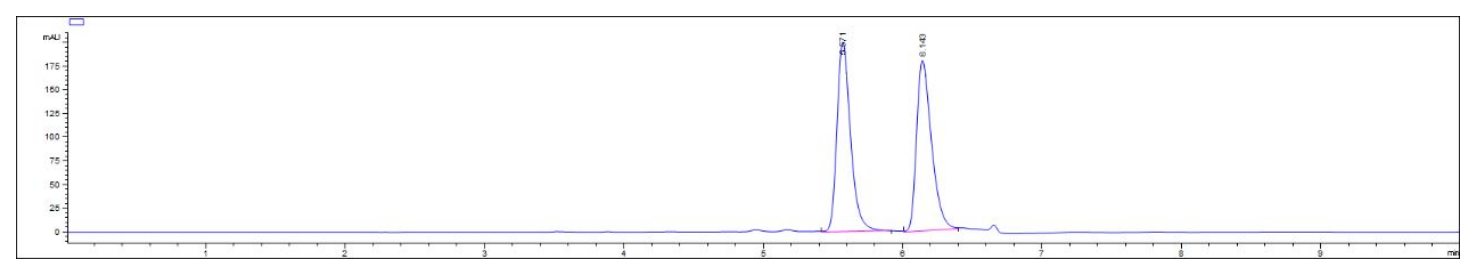

\begin{tabular}{|c|c|c|c|c|c|c|c|}
\hline$\#$ & 时间 & 峰面伿 & 峰高 & 峰宽 & 对称因子 & 峰面积 \% & 类型 \\
\hline 1 & 5.571 & 1363 & 200.6 & 0.1132 & 0.717 & 50.348 & MM \\
\hline 2 & 6.143 & 1344.2 & 180.2 & 0.1243 & 0.63 & 49.652 & MM \\
\hline
\end{tabular}
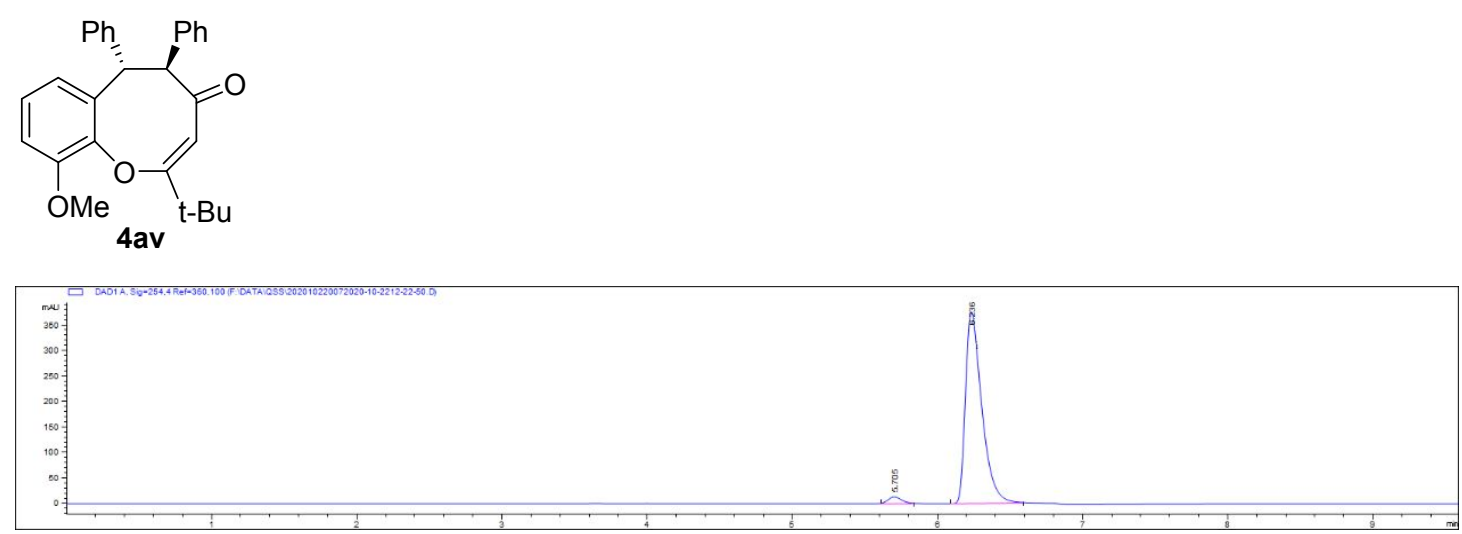

\begin{tabular}{|c|c|c|c|c|c|c|c|}
\hline$\#$ & 时间 & 㠎面积 & 峰高 & 峰宽 & 对称因子 & 峰面积 \% & 类型 \\
\hline 1 & 5.705 & 100.8 & 14.3 & 0.1174 & 0.789 & 3.304 & MM \\
\hline 2 & 6.236 & 2950.9 & 376.5 & 0.1306 & 0.55 & 96.696 & MM \\
\hline
\end{tabular}

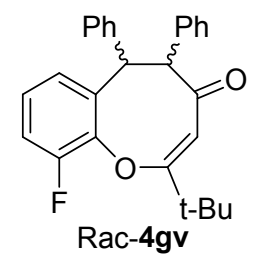

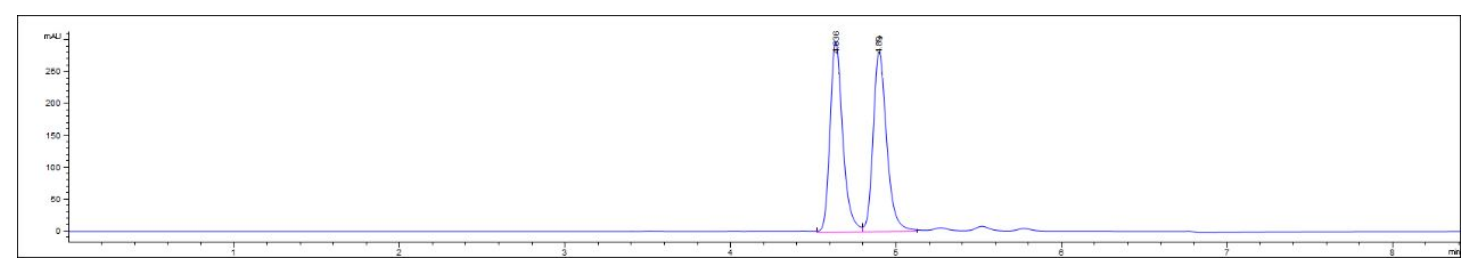

\begin{tabular}{|c|c|c|c|c|c|c|c|}
\hline$\#$ & 时间 & 峰面积 & 峰高 & 峰宽 & 对称因子 & 峰面积 \% & 类型 \\
\hline 1 & 4.636 & 1615.5 & 301.7 & 0.0892 & 0.773 & 49.965 & MF \\
\hline 2 & 4.899 & 1617.7 & 283.2 & 0.0952 & 0.761 & 50.035 & FM \\
\hline
\end{tabular}

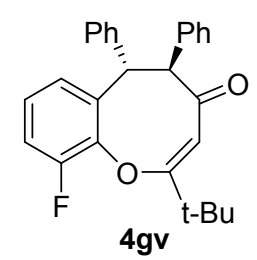




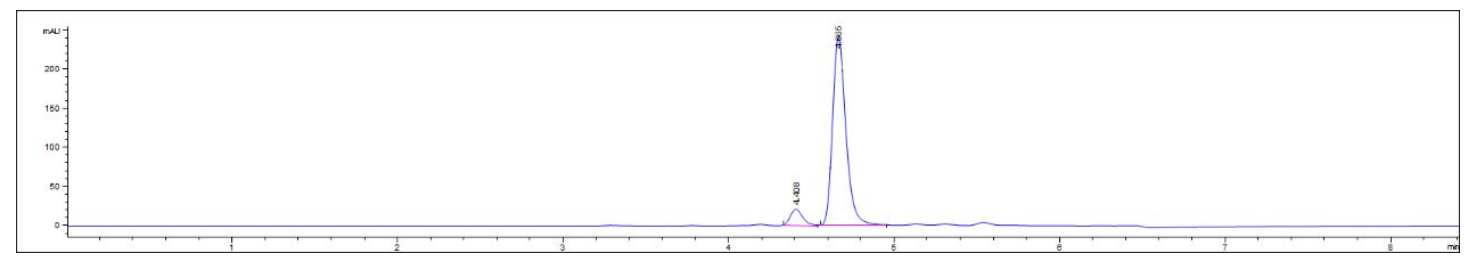

\begin{tabular}{|c|c|c|c|c|c|c|c|}
\hline$\#$ & 时间 & 峰面保 & 峰高 & 峰宽 & 对称因子 & 峰面积 \% & 类型 \\
\hline 1 & 4.408 & 112.7 & 21.3 & 0.0884 & 0.74 & 7.697 & MM \\
\hline 2 & 4.665 & 1351.6 & 242.2 & 0.093 & 0.755 & 92.303 & MM \\
\hline
\end{tabular}
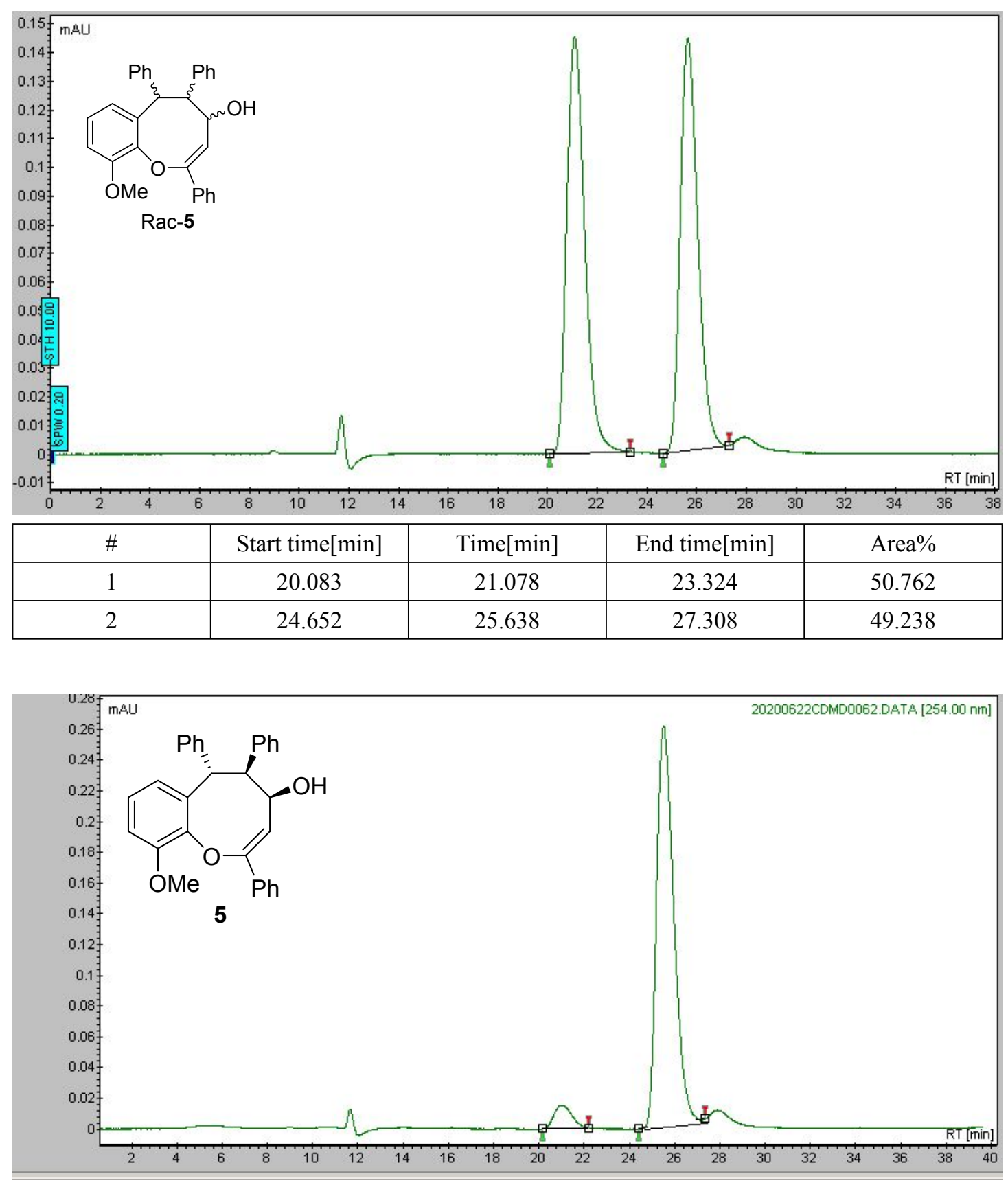

\begin{tabular}{|c|c|c|c|c|}
\hline$\#$ & Start time[min] & Time[min] & End time[min] & Area\% \\
\hline 1 & 20.178 & 21.012 & 22.224 & 3.697 \\
\hline 2 & 24.435 & 25.518 & 27.365 & 97.303 \\
\hline
\end{tabular}



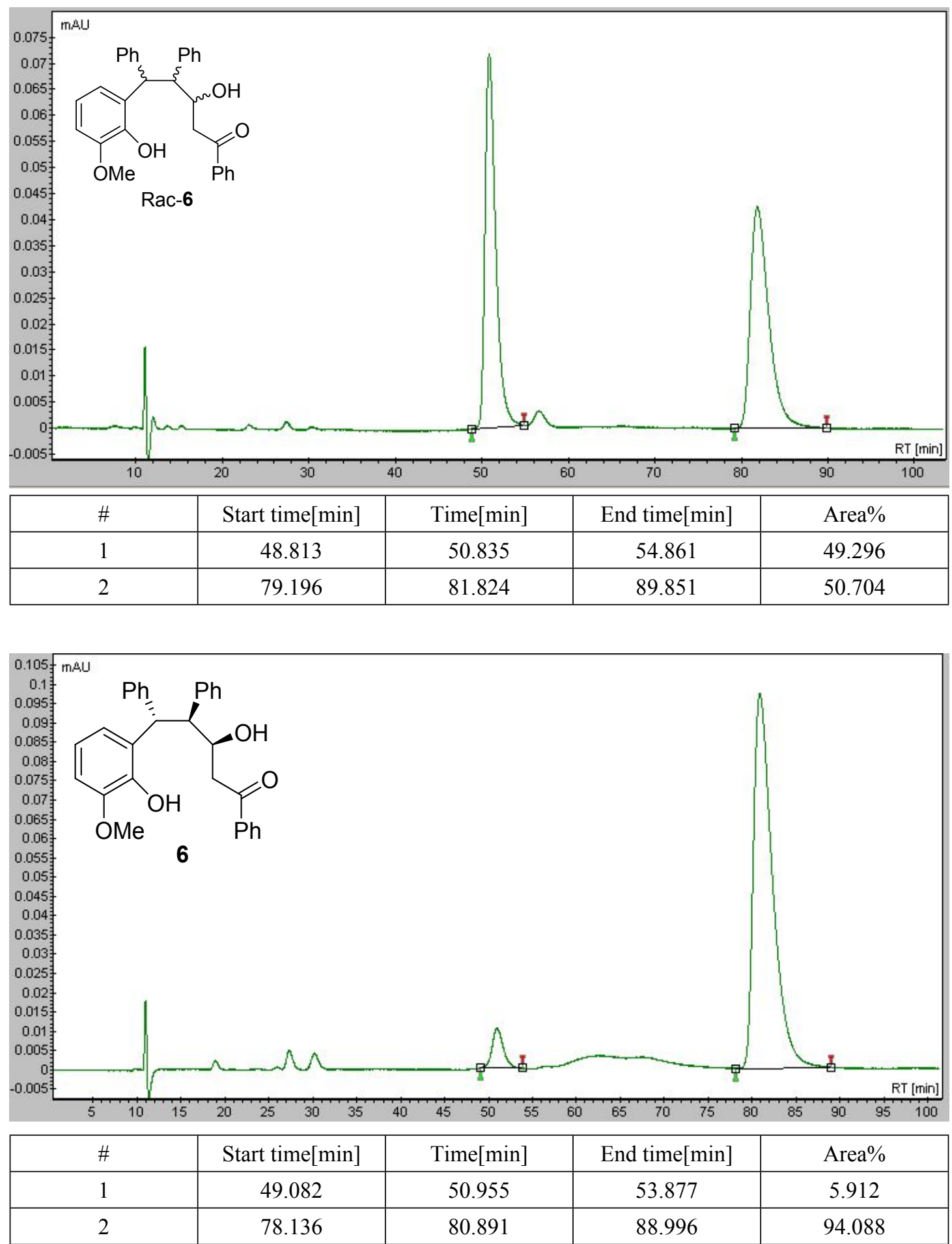


\section{9. ${ }^{1} \mathrm{H}$ NMR and ${ }^{13} \mathrm{C}$ NMR spectra}

${ }^{1} \mathrm{H}$ NMR of compound $2 \mathrm{~d}\left(600 \mathrm{MHz}\right.$ in $\left.\mathrm{CDCl}_{3}\right)$

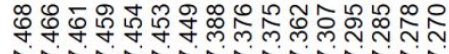<smiles>Cc1ccccc1CC(=O)C#Cc1ccccc1</smiles>

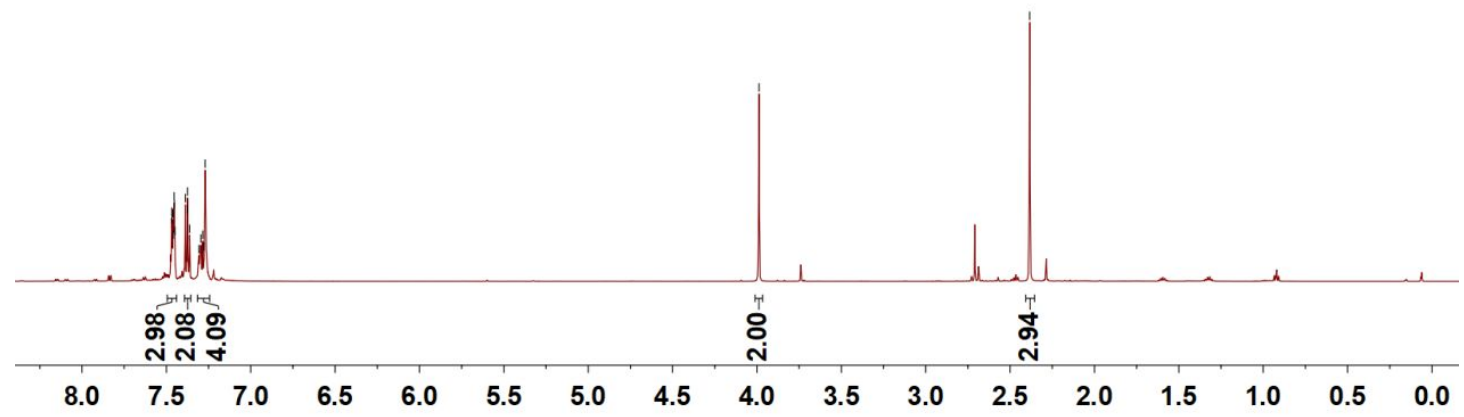

${ }^{13} \mathrm{C}$ NMR of compound $2 \mathrm{~d}\left(151 \mathrm{MHz}\right.$ in $\left.\mathrm{CDCl}_{3}\right)$

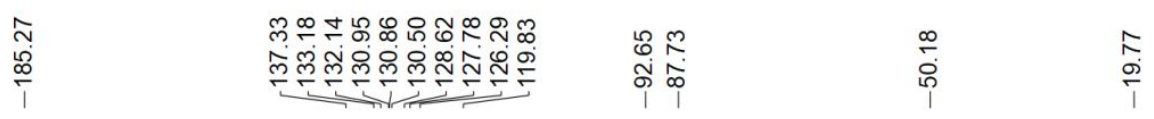<smiles>Cc1ccccc1CC(=O)C#Cc1ccccc1</smiles>

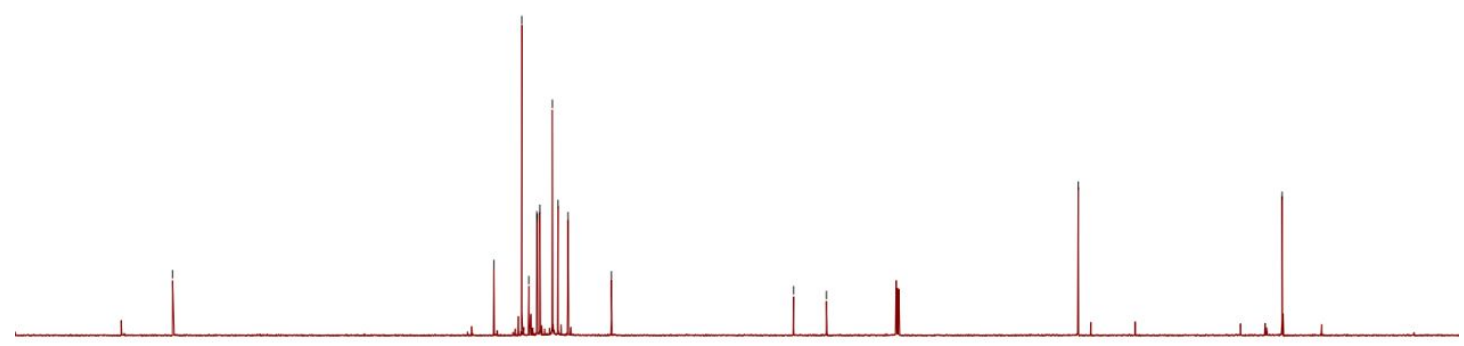

$\begin{array}{lllllllllllllllllllll}200 & 190 & 180 & 170 & 160 & 150 & 140 & 130 & 120 & 110 & 100 & 90 & 80 & 70 & 60 & 50 & 40 & 30 & 20 & 10 & 0\end{array}$ 
${ }^{1} \mathrm{H}$ NMR of compound $2 \mathrm{f}\left(600 \mathrm{MHz}\right.$ in $\left.\mathrm{CDCl}_{3}\right)$

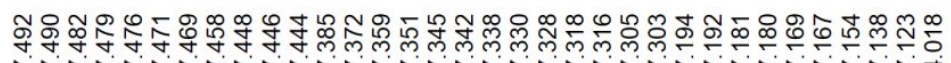

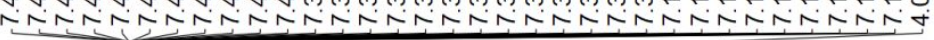

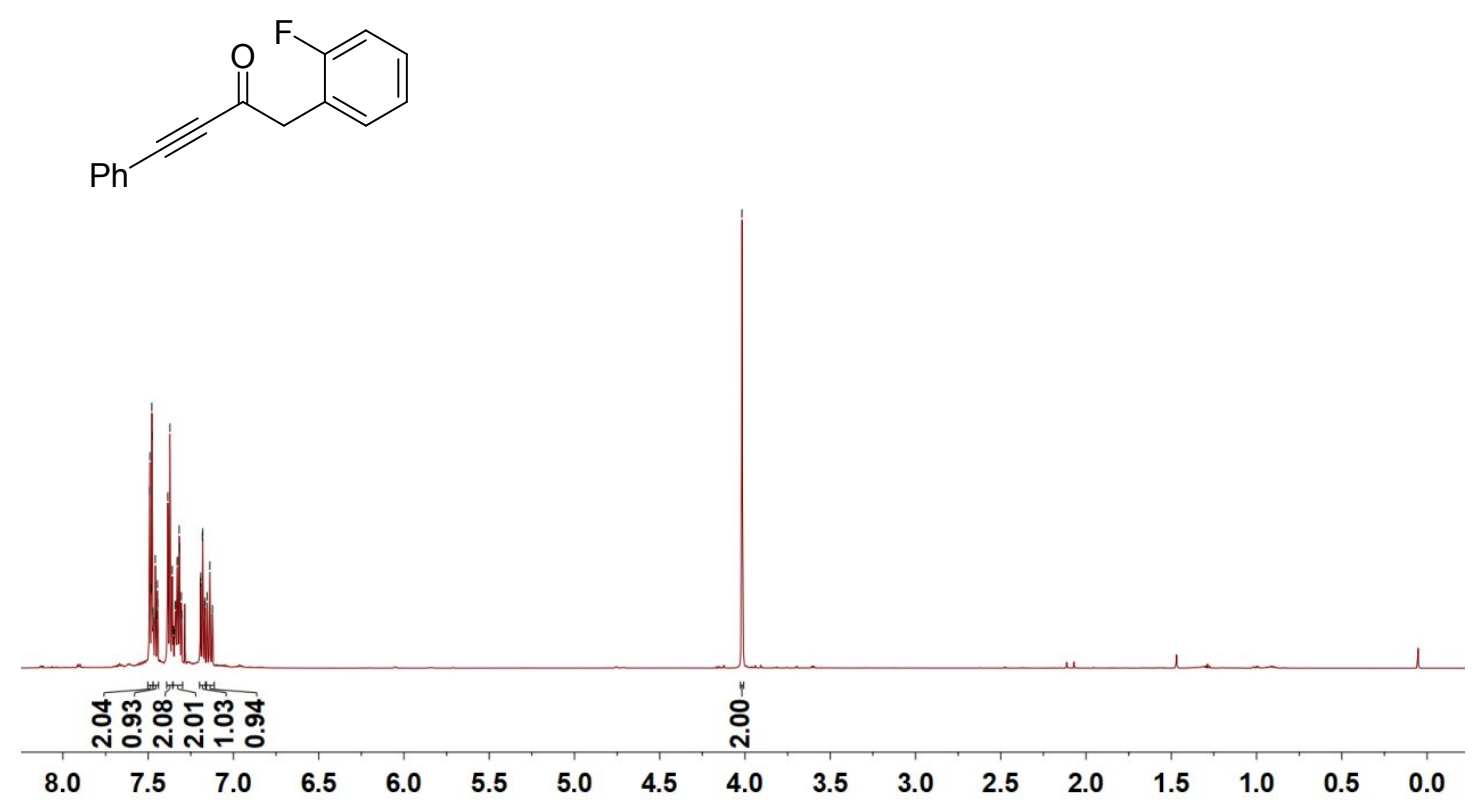

${ }^{13} \mathrm{C}$ NMR of compound $2 \mathrm{f}\left(151 \mathrm{MHz}\right.$ in $\left.\mathrm{CDCl}_{3}\right)$

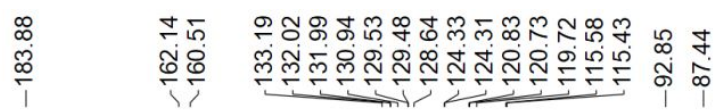

重<smiles>O=C(C#Cc1ccccc1)Cc1ccccc1F</smiles>

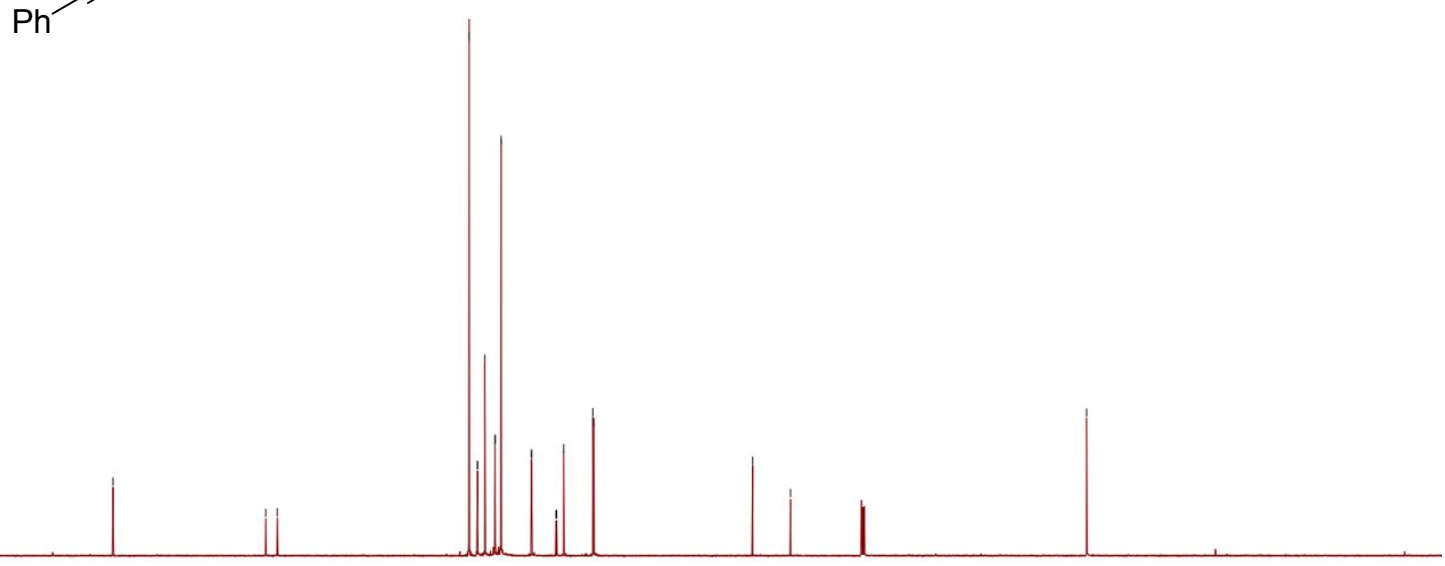

$\begin{array}{lllllllllllllllllllll}\text { J0 } & 190 & 180 & 170 & 160 & 150 & 140 & 130 & 120 & 110 & 100 & 90 & 80 & 70 & 60 & 50 & 40 & 30 & 20 & 10 & 0\end{array}$ 
${ }^{1} \mathrm{H}$ NMR of compound $2 \mathrm{i}\left(600 \mathrm{MHz}\right.$ in $\left.\mathrm{CDCl}_{3}\right)$

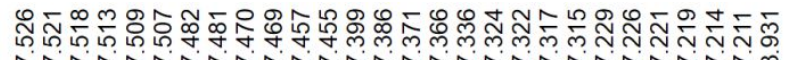

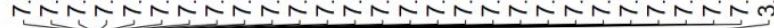

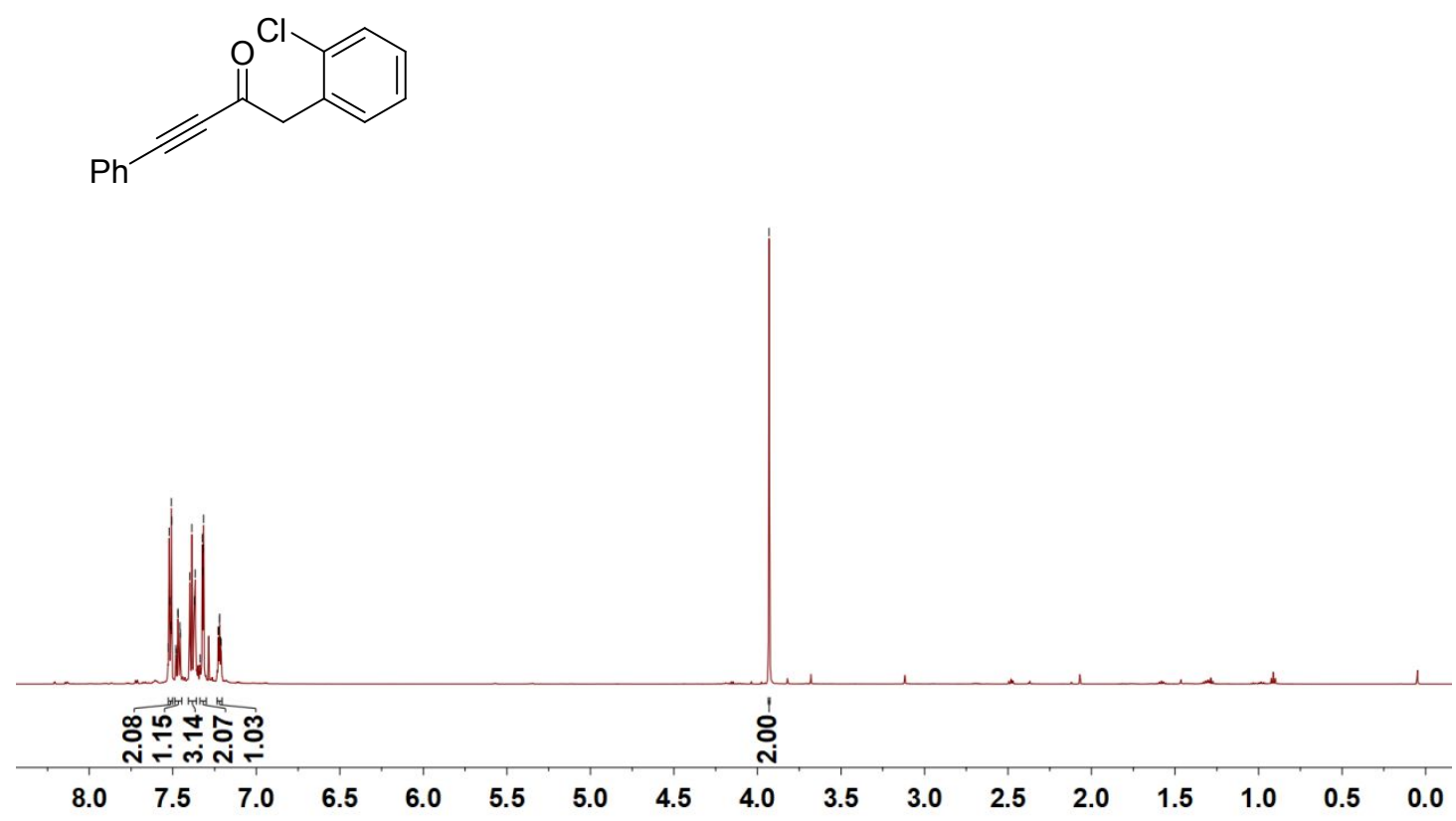

${ }^{13} \mathrm{C}$ NMR of compound $2 \mathrm{i}\left(151 \mathrm{MHz}\right.$ in $\left.\mathrm{CDCl}_{3}\right)$

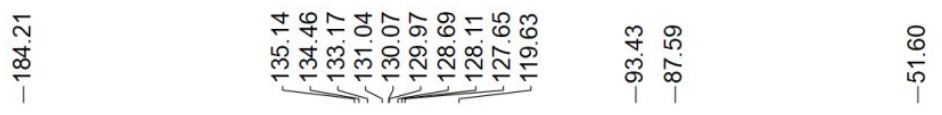

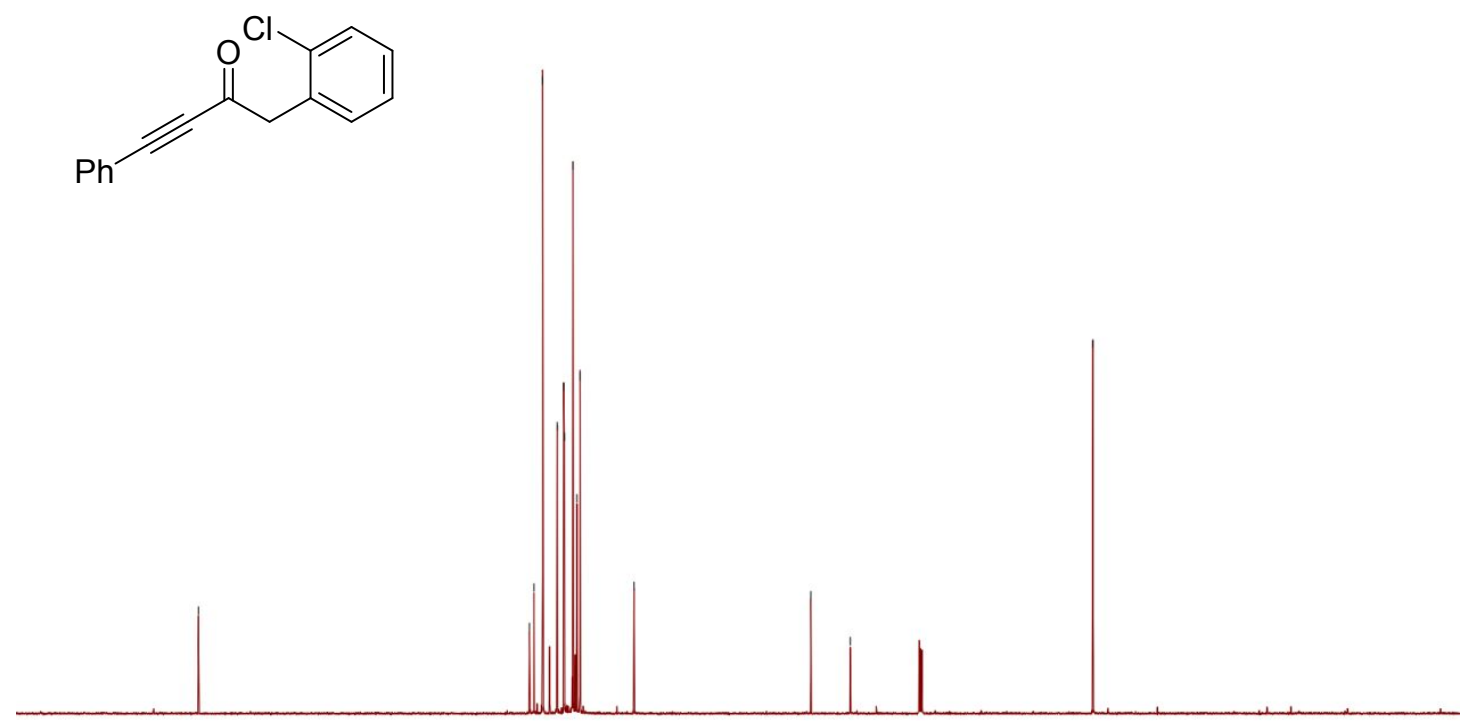

$\begin{array}{llllllllllllllllllllll}10 & 200 & 190 & 180 & 170 & 160 & 150 & 140 & 130 & 120 & 110 & 100 & 90 & 80 & 70 & 60 & 50 & 40 & 30 & 20 & 10 & 0\end{array}$ 
${ }^{1} \mathrm{H}$ NMR of compound $2 \mathrm{q}\left(600 \mathrm{MHz}\right.$ in $\left.\mathrm{CDCl}_{3}\right)$

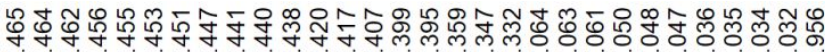

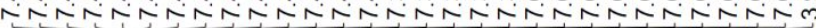<smiles>O=C(C#Cc1ccc(F)cc1)c1ccccc1</smiles>

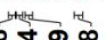
\&े

$\begin{array}{lllllllllllllllll}8.0 & 7.5 & 7.0 & 6.5 & 6.0 & 5.5 & 5.0 & 4.5 & 4.0 & 3.5 & 3.0 & 2.5 & 2.0 & 1.5 & 1.0 & 0.5 & 0.0\end{array}$

${ }^{13} \mathrm{C}$ NMR of compound $2 \mathrm{q}\left(151 \mathrm{MHz}\right.$ in $\left.\mathrm{CDCl}_{3}\right)$

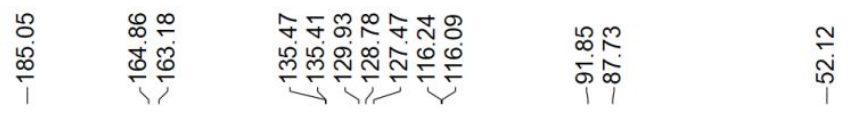<smiles>O=C(C#Cc1ccc(F)cc1)Cc1ccccc1</smiles>

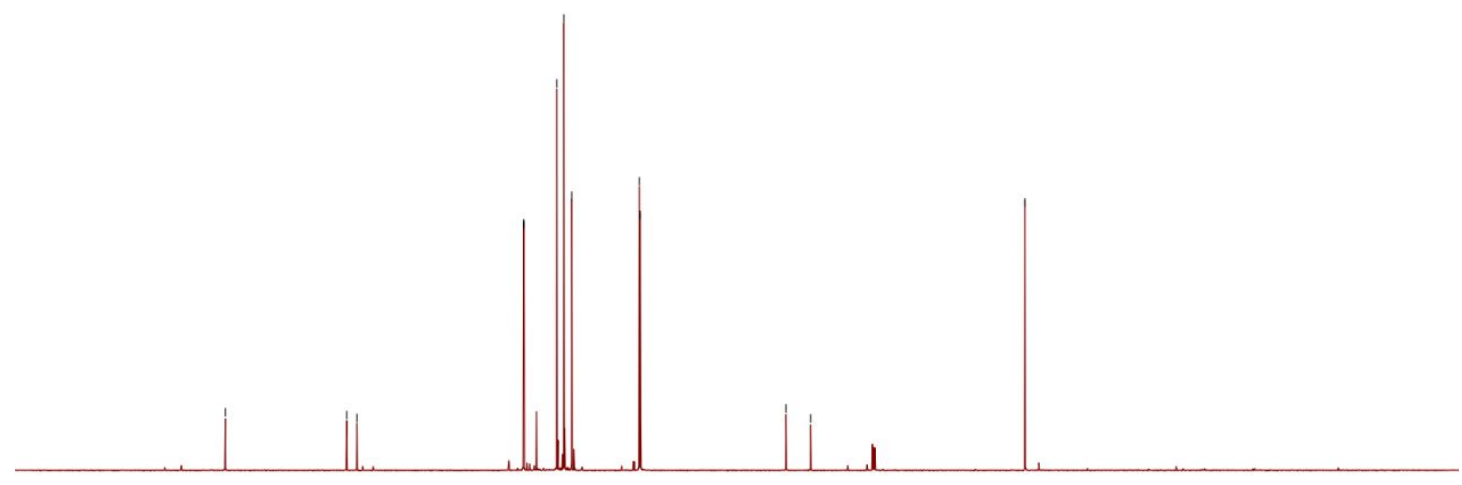

$\begin{array}{lllllllllllllllllllllllll}20 & 210 & 200 & 190 & 180 & 170 & 160 & 150 & 140 & 130 & 120 & 110 & 100 & 90 & 80 & 70 & 60 & 50 & 40 & 30 & 20 & 10 & 0 & -10 & -2\end{array}$ 
${ }^{1} \mathrm{H}$ NMR of compound 2r (600 $\mathrm{MHz}$ in $\left.\mathrm{CDCl}_{3}\right)$

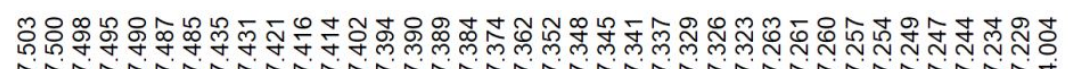

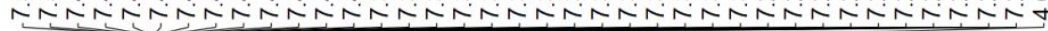<smiles>O=C(C#Cc1ccccc1Cl)c1ccccc1</smiles>

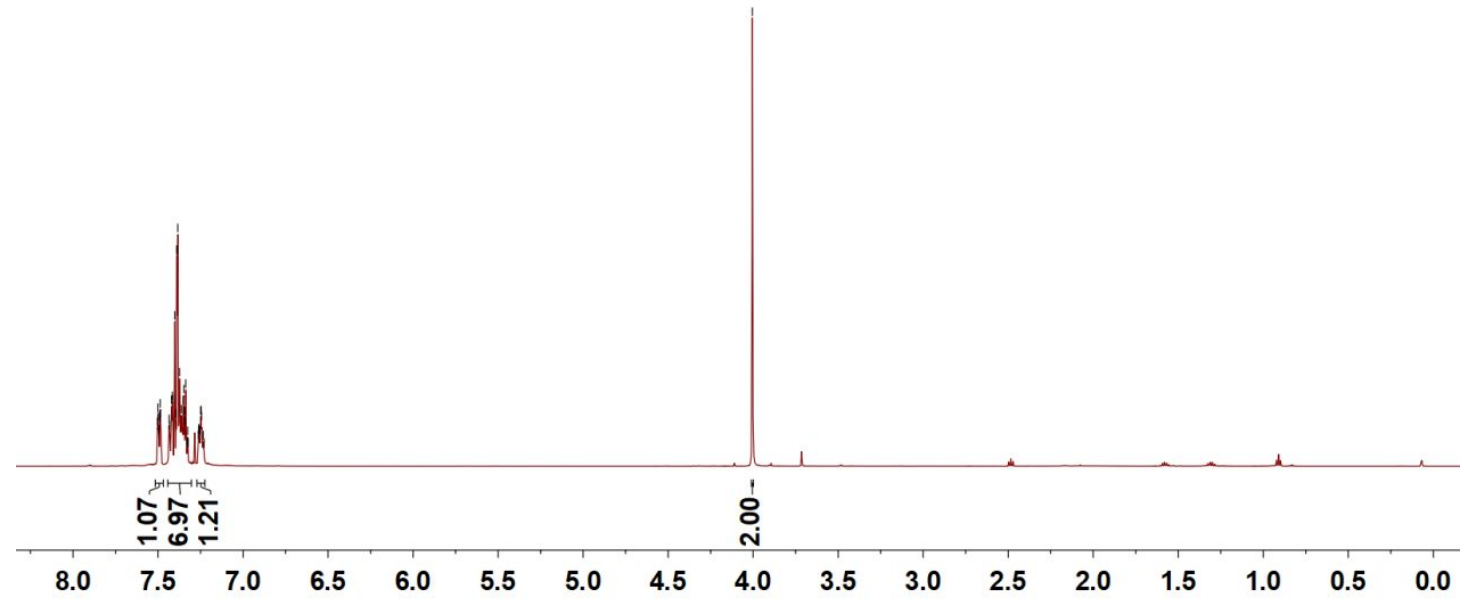

${ }^{13} \mathrm{C}$ NMR of compound 2r (151 $\mathrm{MHz}$ in $\left.\mathrm{CDCl}_{3}\right)$

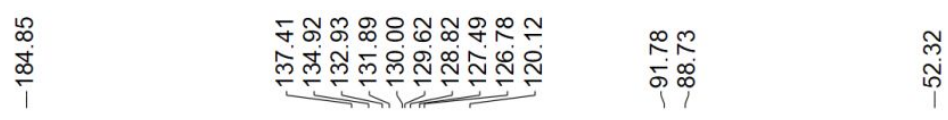<smiles>O=C(C#Cc1ccccc1Cl)c1ccccc1</smiles>

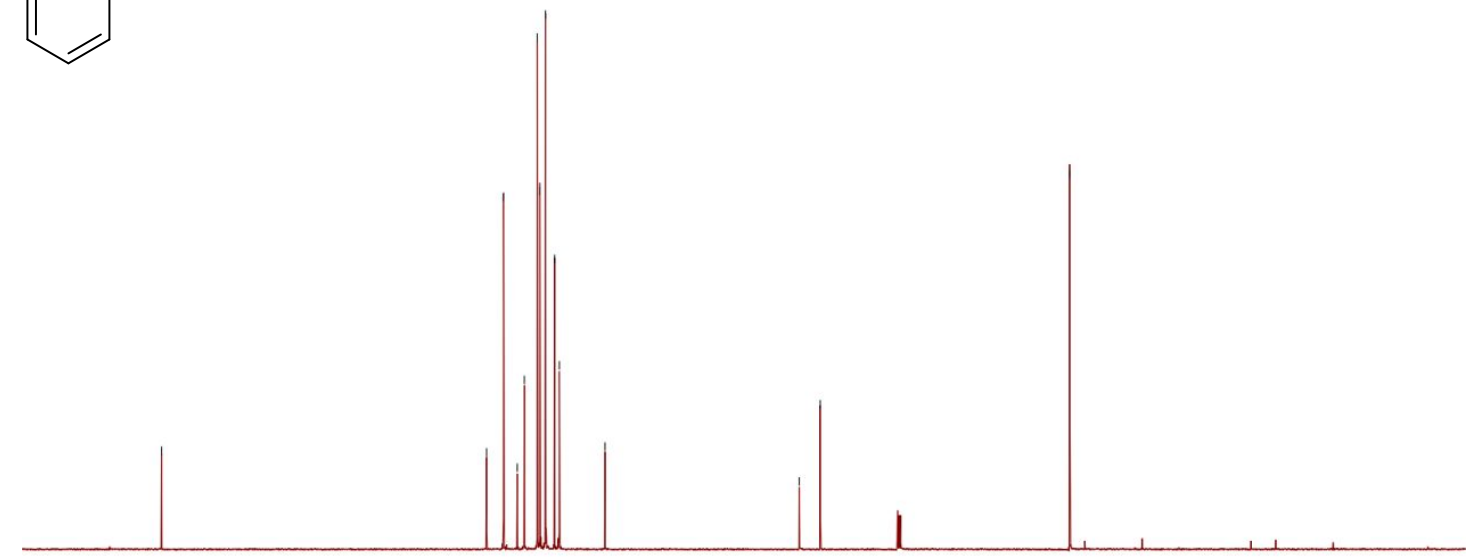

$\begin{array}{lllllllllllllllllllll}200 & 190 & 180 & 170 & 160 & 150 & 140 & 130 & 120 & 110 & 100 & 90 & 80 & 70 & 60 & 50 & 40 & 30 & 20 & 10 & 0\end{array}$ 
${ }^{1} \mathrm{H}$ NMR of compound 2s (600 $\mathrm{MHz}$ in $\left.\mathrm{CDCl}_{3}\right)$

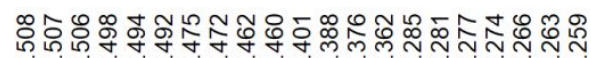

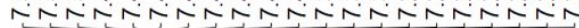<smiles>O=C(C#Cc1cccc(Cl)c1)Cc1ccccc1</smiles>

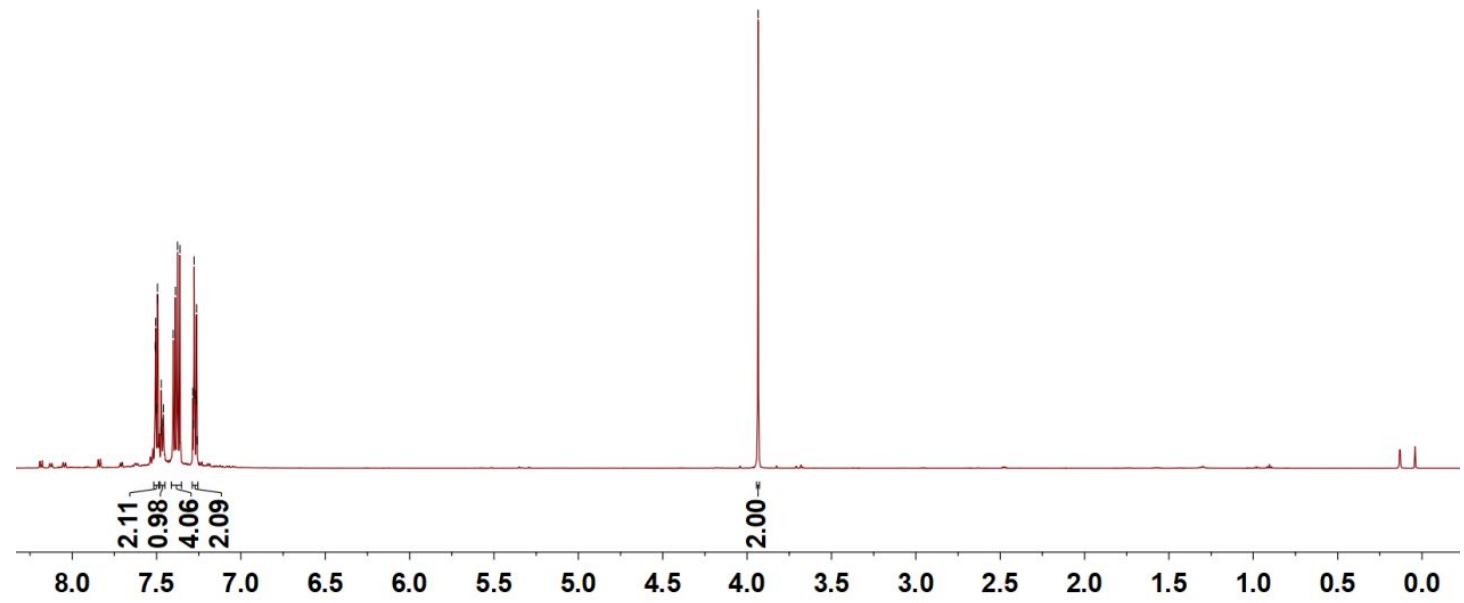

${ }^{13} \mathrm{C}$ NMR of compound 2s (151 $\mathrm{MHz}$ in $\left.\mathrm{CDCl}_{3}\right)$

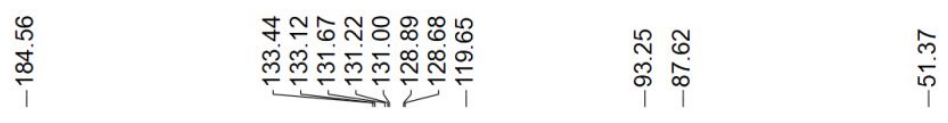<smiles>O=C(C#Cc1cccc(Cl)c1)c1ccccc1</smiles>

in

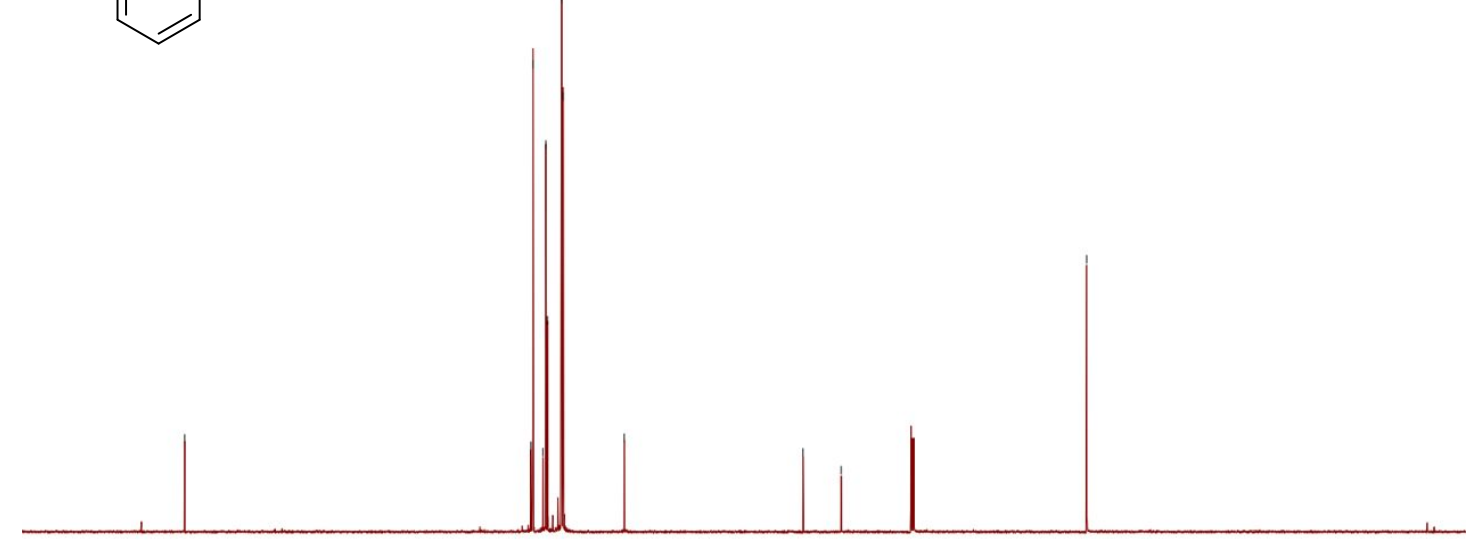

$\begin{array}{lllllllllllllllllllll}200 & 190 & 180 & 170 & 160 & 150 & 140 & 130 & 120 & 110 & 100 & 90 & 80 & 70 & 60 & 50 & 40 & 30 & 20 & 10 & 0\end{array}$ 
${ }^{1} \mathrm{H}$ NMR of compound 2v (600 $\mathrm{MHz}$ in $\left.\mathrm{CDCl}_{3}\right)$

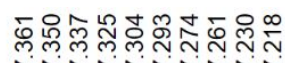

riñNiñNin<smiles>CC(C)(C)C#CC(=O)c1ccccc1</smiles>

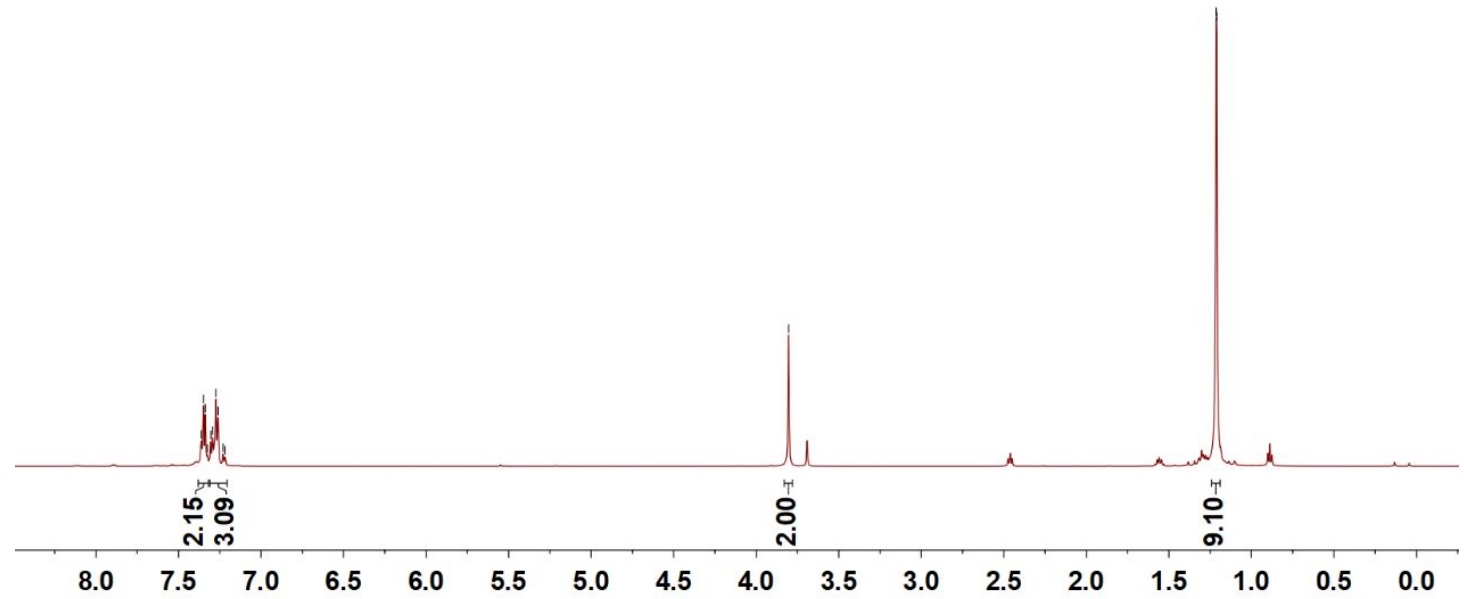

${ }^{13} \mathrm{C}$ NMR of compound 2v (151 $\mathrm{MHz}$ in $\left.\mathrm{CDCl}_{3}\right)$

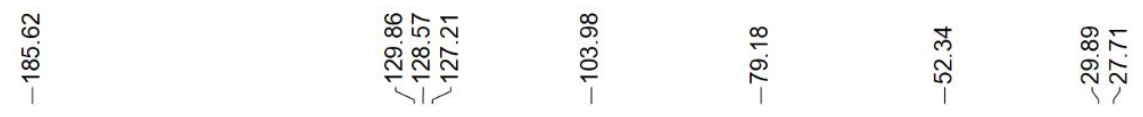<smiles>CC(C)(C)C#CC(=O)c1ccccc1</smiles>

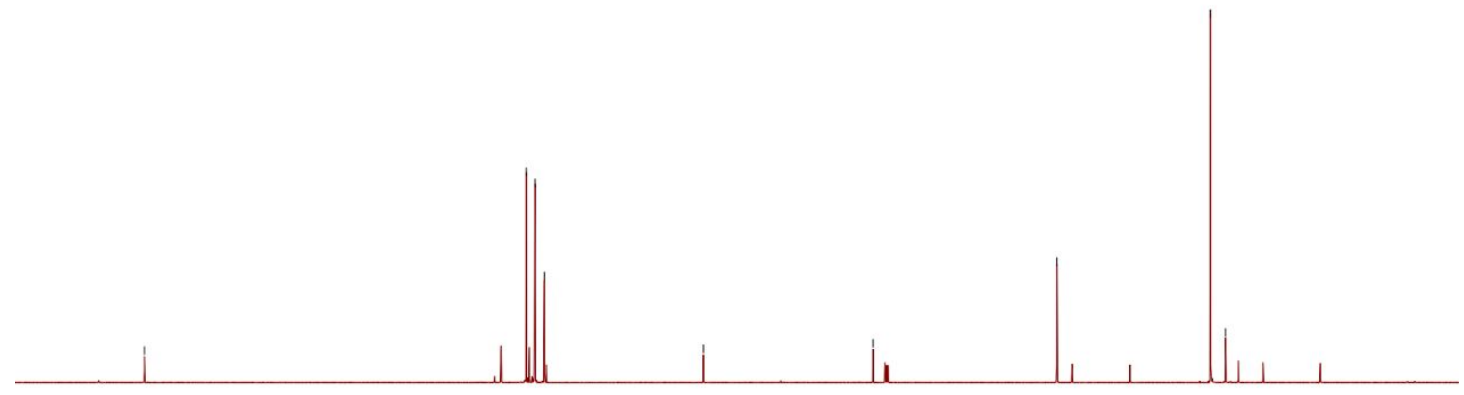

$\begin{array}{lllllllllllllllllllll}200 & 190 & 180 & 170 & 160 & 150 & 140 & 130 & 120 & 110 & 100 & 90 & 80 & 70 & 60 & 50 & 40 & 30 & 20 & 10 & 0\end{array}$ 
${ }^{1} \mathrm{H}$ NMR of compound 3aa (500 $\mathrm{MHz}$ in $\left.\mathrm{CDCl}_{3}\right)$

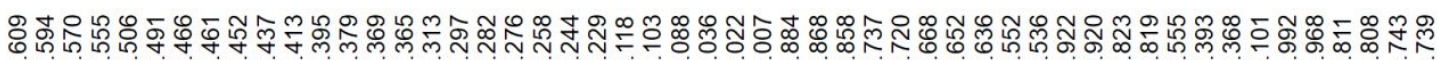

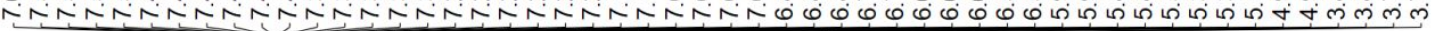<smiles>COc1cccc(C(C(=O)C#Cc2ccccc2)c2ccccc2)c1</smiles>

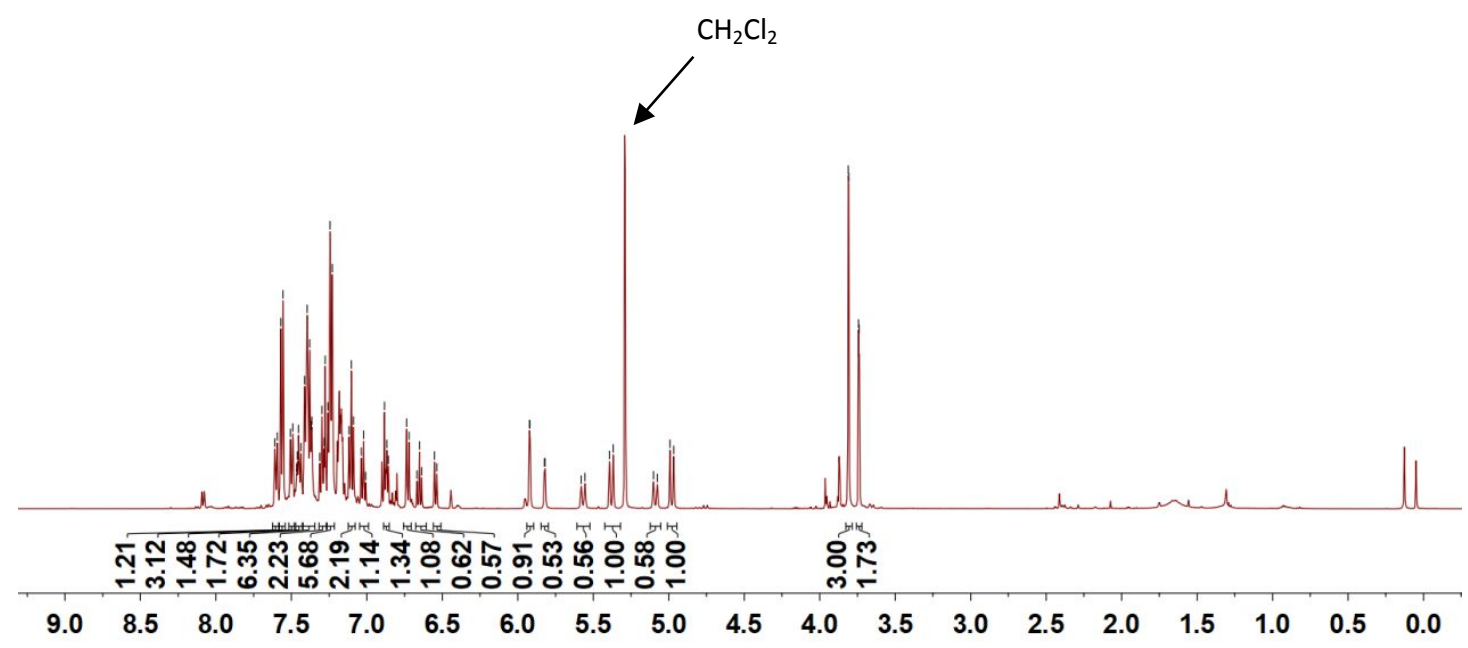

${ }^{13} \mathrm{C}$ NMR of compound 3aa (126 $\mathrm{MHz}$ in $\mathrm{CDCl}_{3}$ )

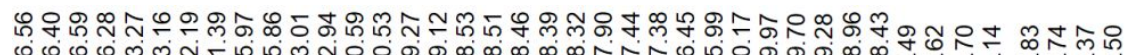

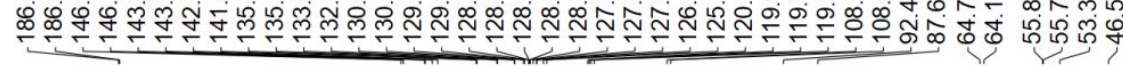<smiles>COc1cccc(C(C(=O)C#CC#Cc2ccccc2)c2ccccc2)c1</smiles> 
${ }^{1} \mathrm{H}$ NMR of compound $4 \mathrm{aa}\left(500 \mathrm{MHz}\right.$ in $\left.\mathrm{CDCl}_{3}\right)$
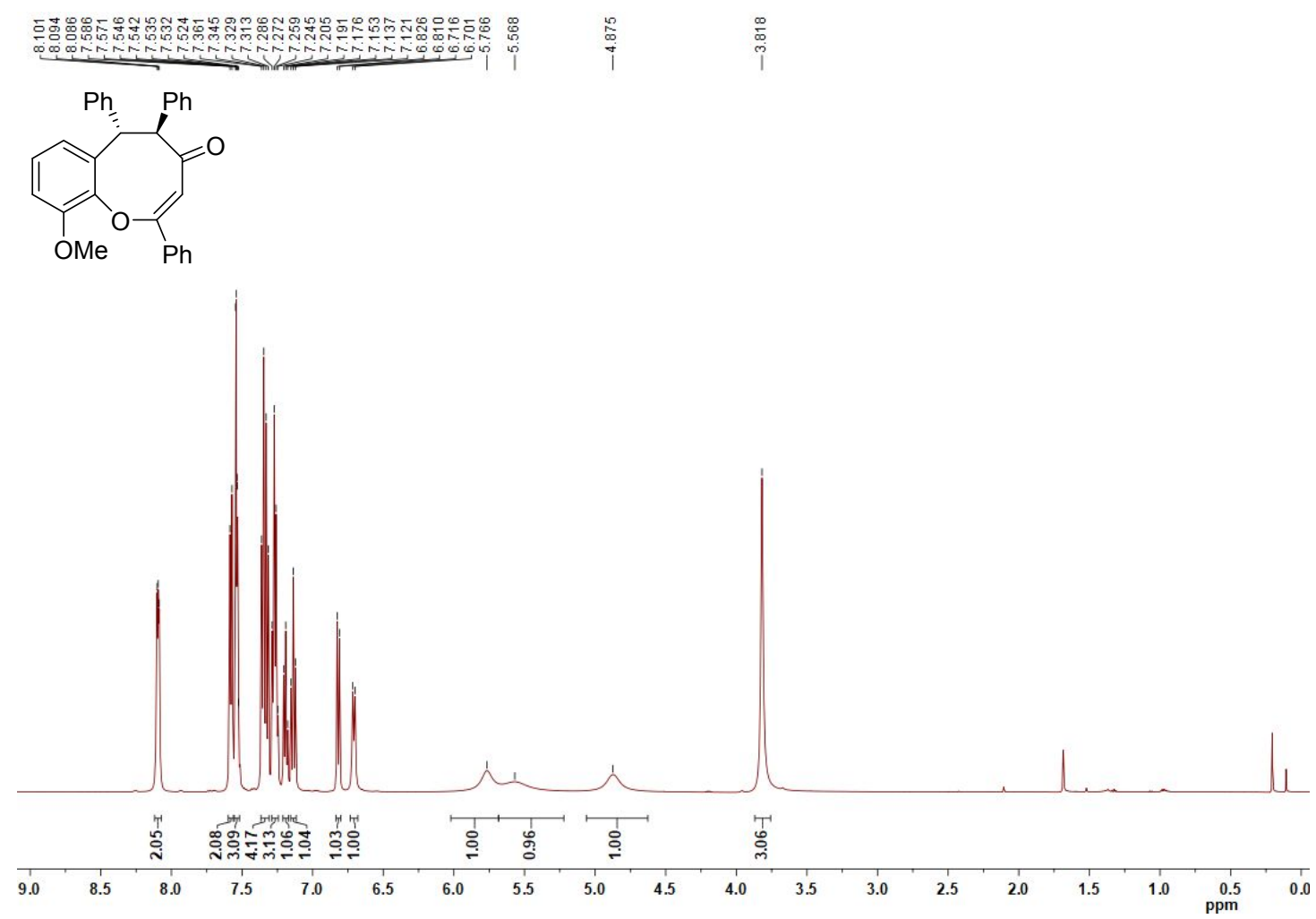

${ }^{13} \mathrm{C}$ NMR of compound $4 \mathrm{aa}\left(126 \mathrm{MHz}\right.$ in $\left.\mathrm{CDCl}_{3}\right)$

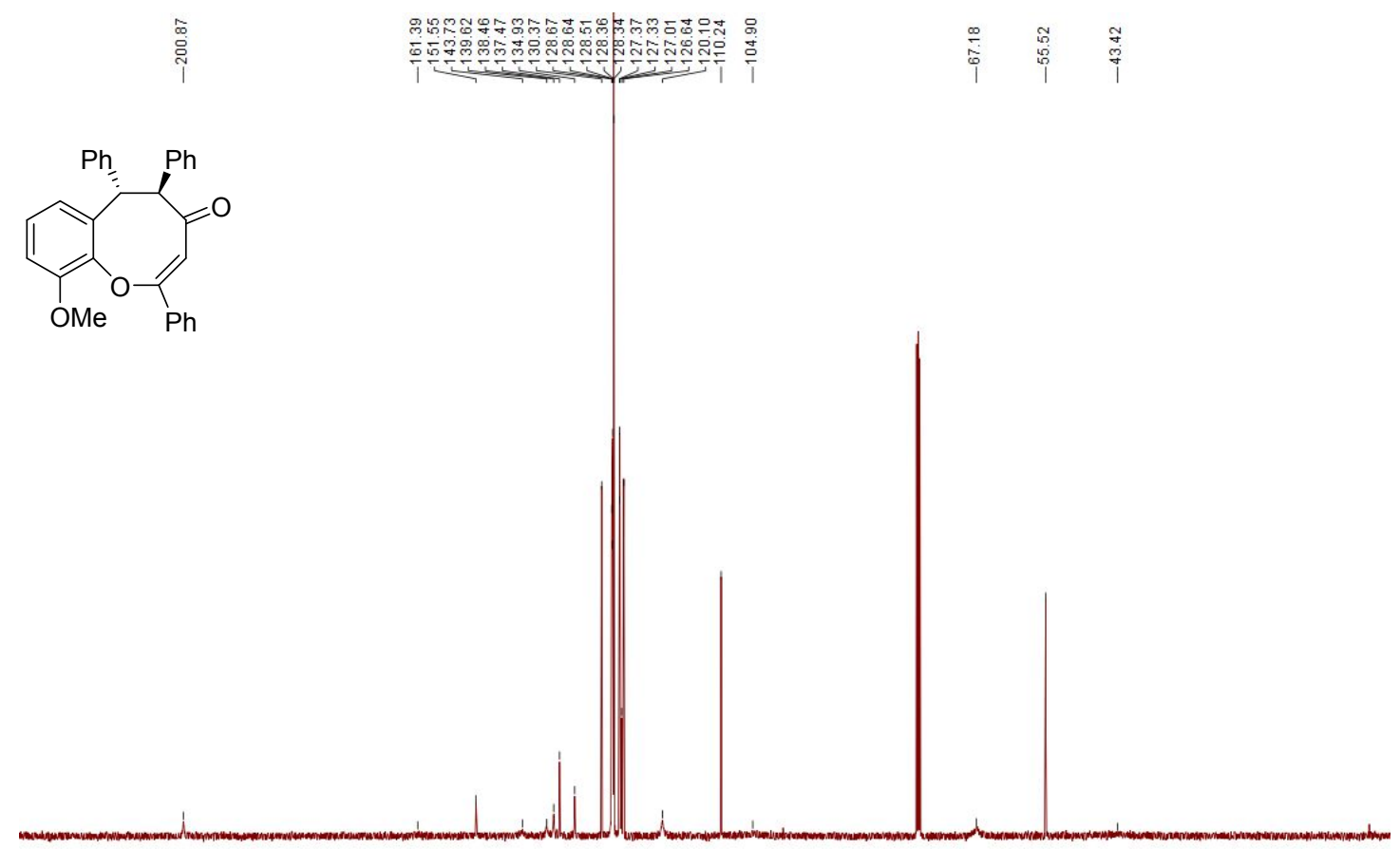

$\begin{array}{lllllllllllllllllllllllllll}1 & 220 & 210 & 200 & 190 & 180 & 170 & 160 & 150 & 140 & 130 & 120 & 110 & 100 & 90 & 80 & 70 & 60 & 50 & 40 & 30 & 20 & 10 & 0 \\ \mathrm{ppm} & \end{array}$ 
${ }^{1} \mathrm{H}$ NMR of compound $4 \mathrm{ba}\left(500 \mathrm{MHz}\right.$ in $\left.\mathrm{CDCl}_{3}\right)$

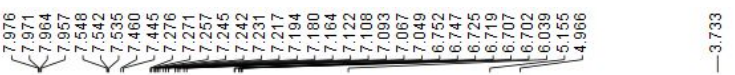<smiles>COc1ccc2c(c1)OC(c1ccccc1)CC(=O)C(c1ccccc1)C(c1ccccc1)C=C2</smiles>

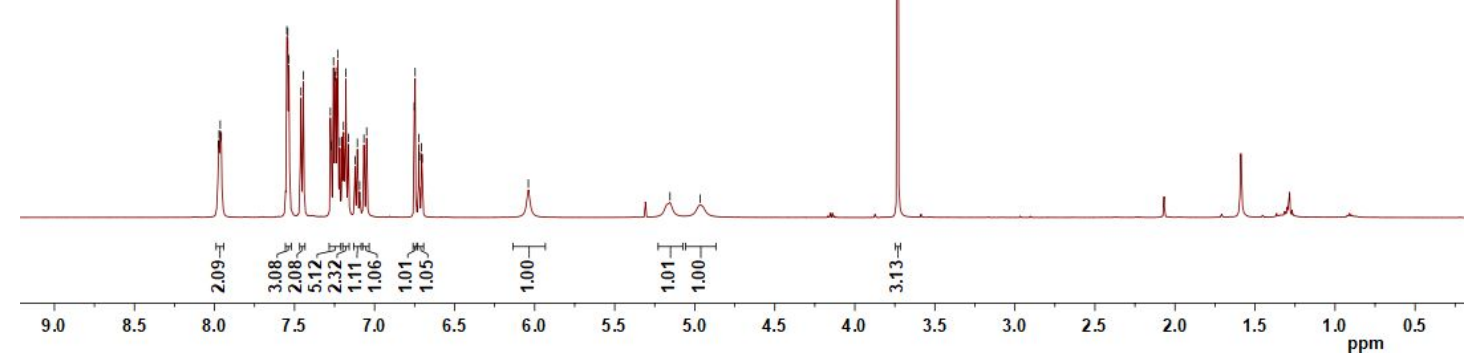

${ }^{13} \mathrm{C}$ NMR of compound $4 \mathrm{ba}\left(126 \mathrm{MHz}\right.$ in $\left.\mathrm{CDCl}_{3}\right)$
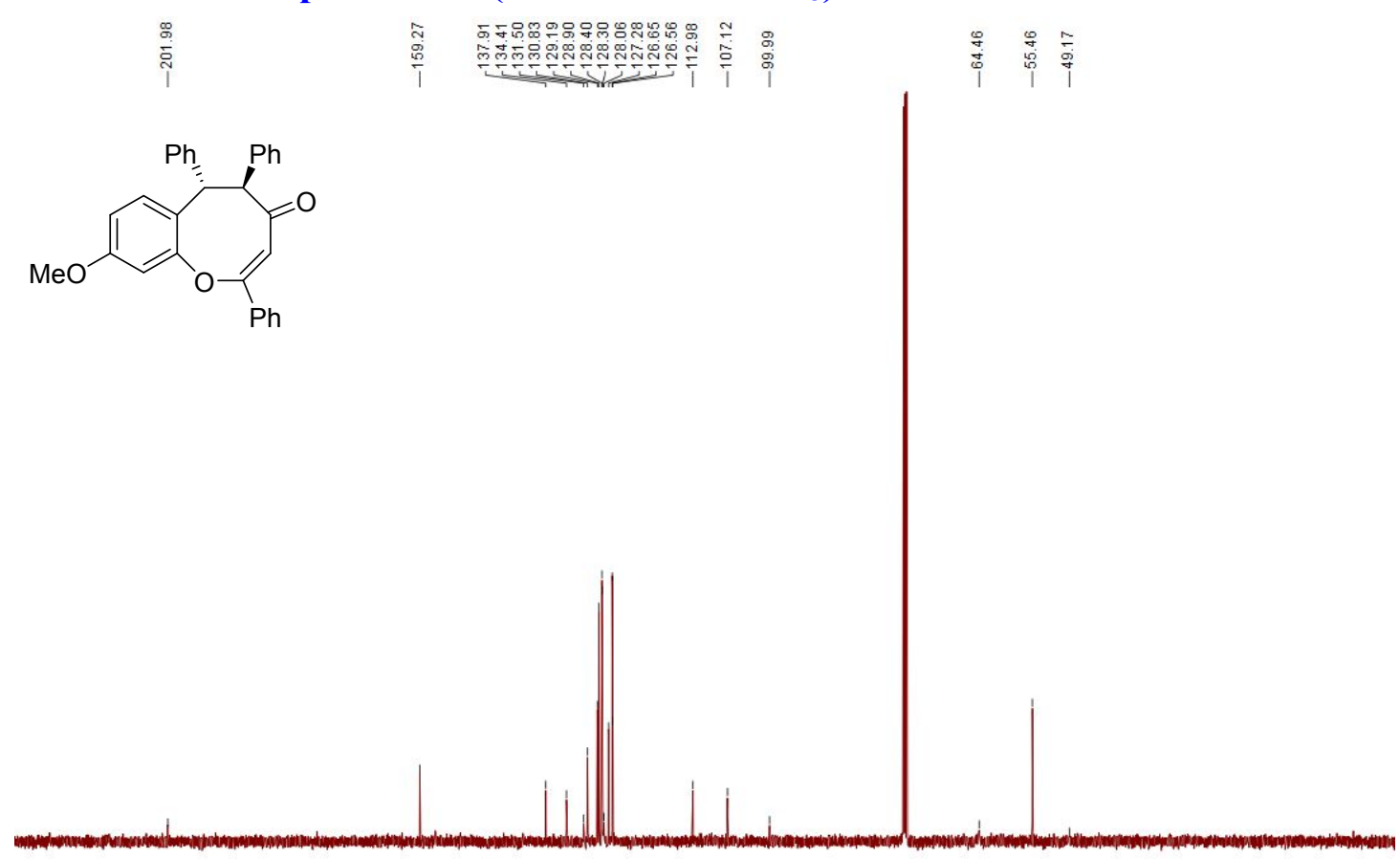

$\begin{array}{lllllllllllllllllllllllllllll} & 120 & 210 & 200 & 190 & 180 & 170 & 160 & 150 & 140 & 130 & 120 & 110 & 100 & 90 & 80 & 70 & 60 & 50 & 40 & 30 & 20 & 10 & 0\end{array}$ 
${ }^{1} \mathrm{H}$ NMR of compound $4 \mathrm{ca}\left(500 \mathrm{MHz}\right.$ in $\left.\mathrm{CDCl}_{3}\right)$
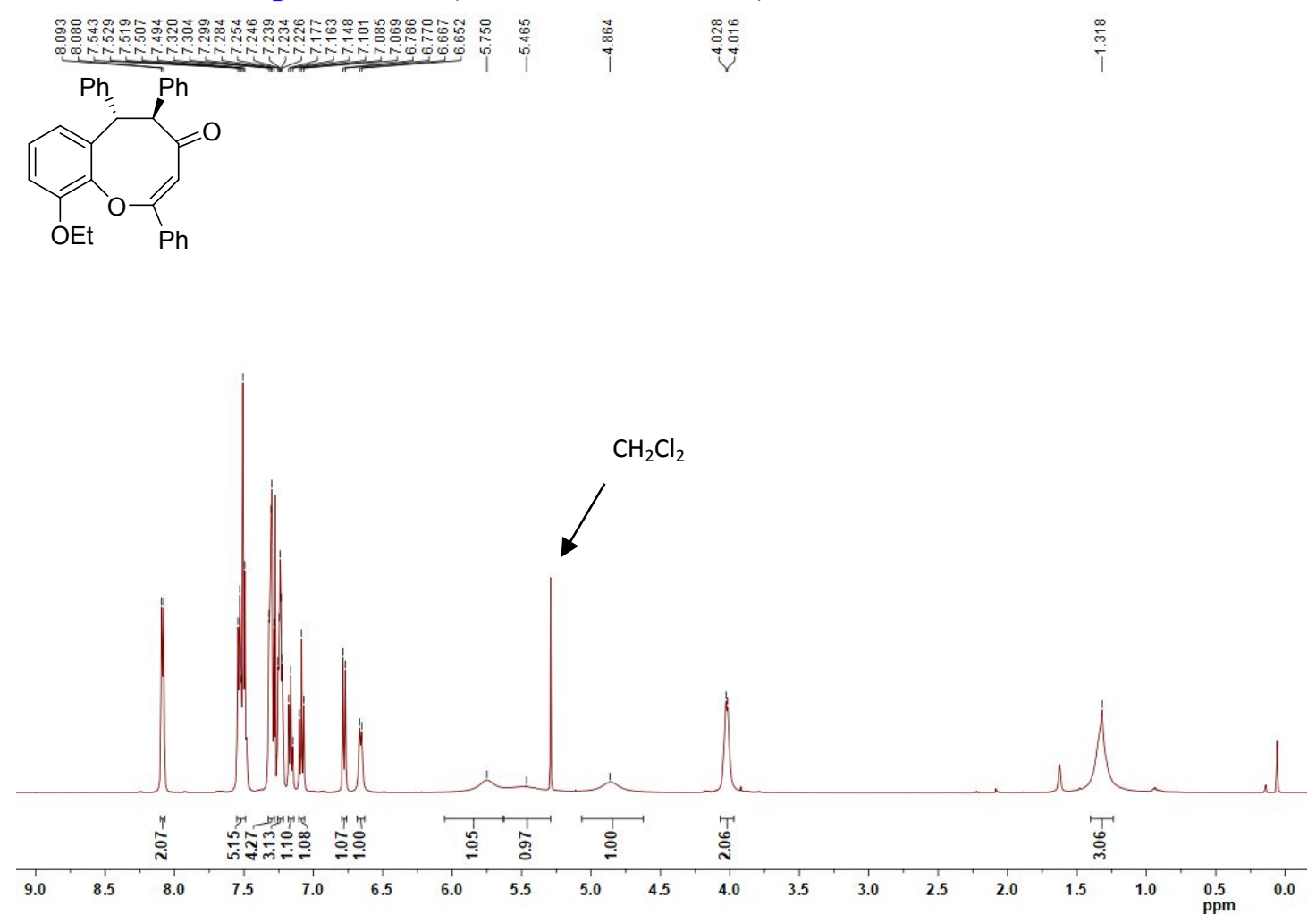

${ }^{13} \mathrm{C}$ NMR of compound $4 \mathrm{ca}\left(126 \mathrm{MHz}\right.$ in $\left.\mathrm{CDCl}_{3}\right)$
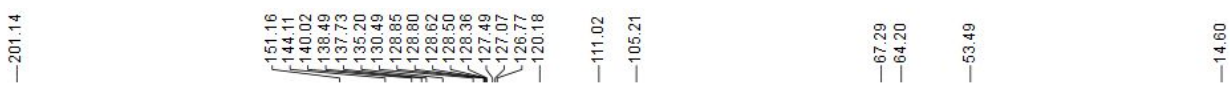<smiles>CCOc1cccc2c1OC(c1ccccc1)CC(=O)C(c1ccccc1)C2c1ccccc1</smiles> 
${ }^{1} \mathrm{H}$ NMR of compound 4da (500 $\mathrm{MHz}$ in $\left.\mathrm{CDCl}_{3}\right)$

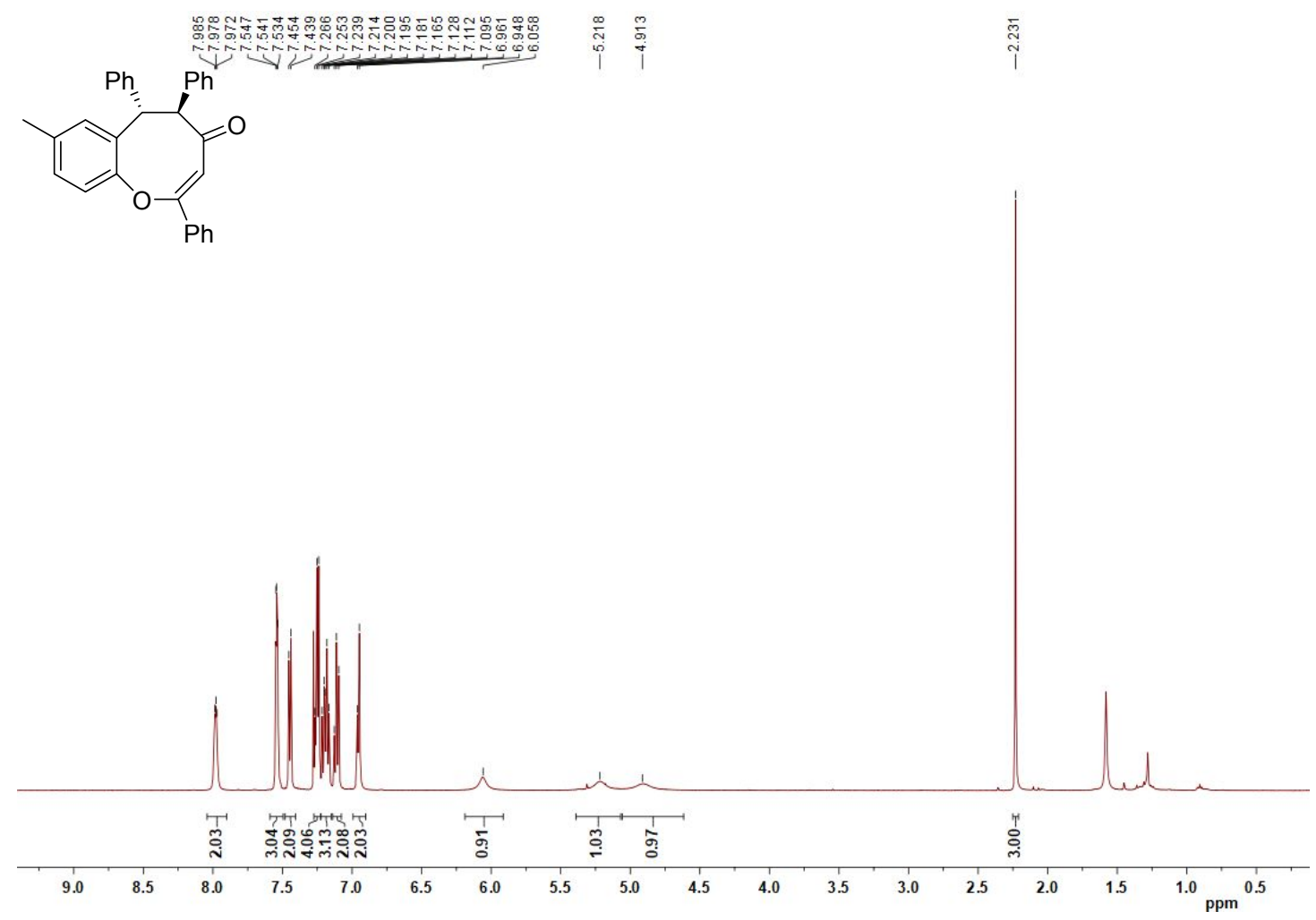

${ }^{13} \mathrm{C}$ NMR of compound $4 \mathrm{da}\left(126 \mathrm{MHz}\right.$ in $\left.\mathrm{CDCl}_{3}\right)$

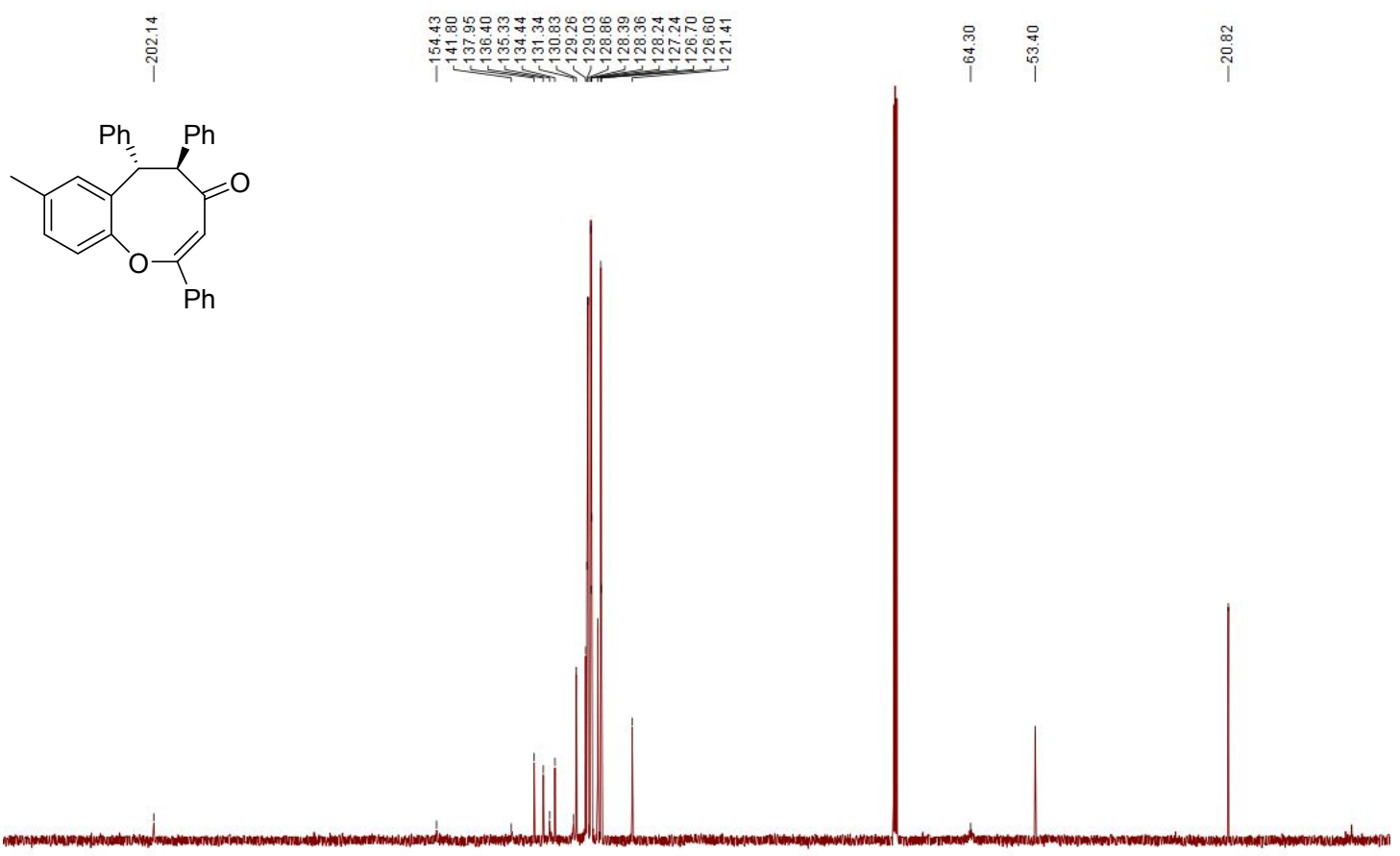

$\begin{array}{llllllllllllllllllllllllllllllllll} & 120 & 210 & 200 & 190 & 180 & 170 & 160 & 150 & 140 & 130 & 120 & 110 & 100 & 90 & 80 & 70 & 60 & 50 & 40 & 30 & 20 & 10 & 0 \\ \mathrm{ppm} & \end{array}$ 
${ }^{1} \mathrm{H}$ NMR of compound 4ea (500 $\mathrm{MHz}$ in $\left.\mathrm{CDCl}_{3}\right)$

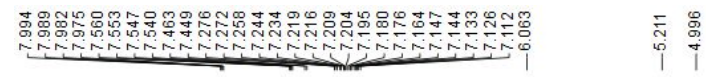<smiles>O=C1/C=C(\c2ccccc2)C(c2ccccc2)[C@H](c2ccccc2)c2ccccc2O1</smiles>

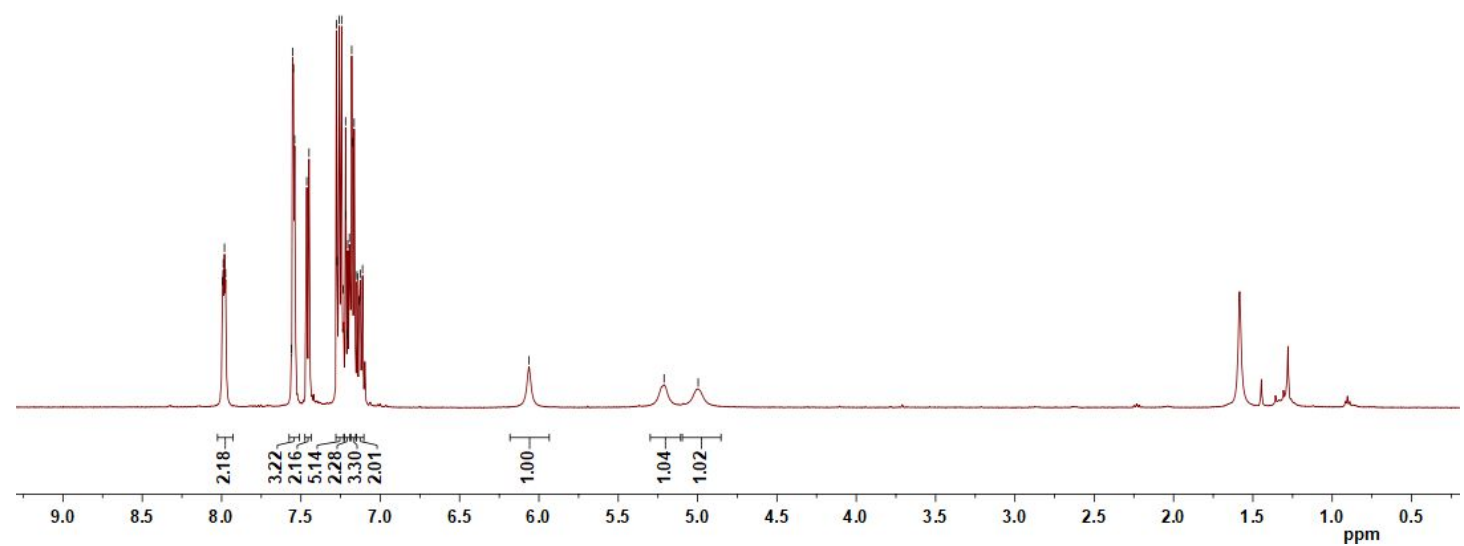

${ }^{13} \mathrm{C}$ NMR of compound 4ea (126 $\mathrm{MHz}$ in $\left.\mathrm{CDCl}_{3}\right)$

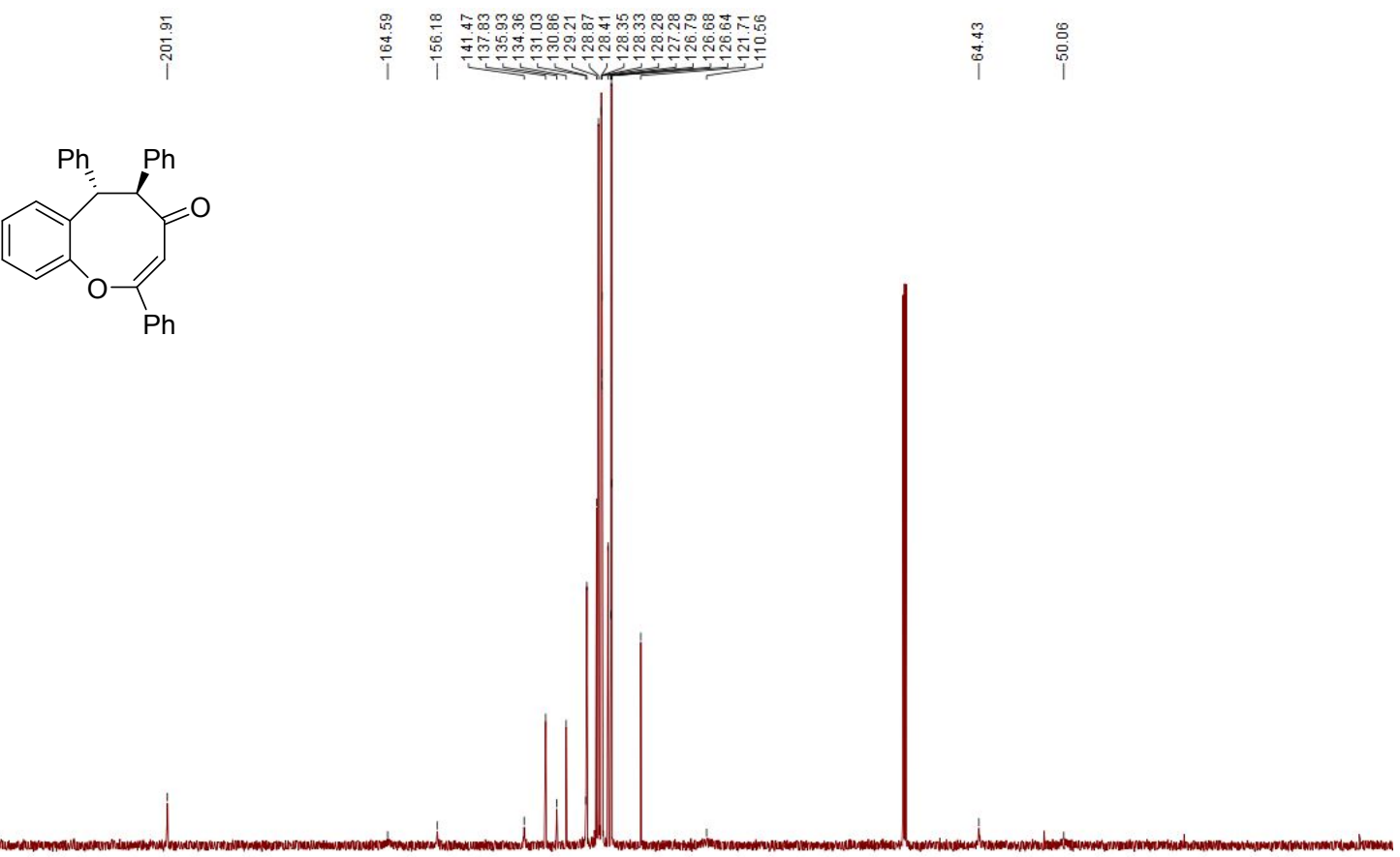

\begin{tabular}{|llllllllllllllllllllllllllll}
\hline 30 & 220 & 210 & 200 & 190 & 180 & 170 & 160 & 150 & 140 & 130 & 120 & 110 & 100 & 90 & 80 & 70 & 60 & 50 & 40 & 30 & 20 & 10 & 0 \\
$\mathrm{ppm}$ & &
\end{tabular} 
${ }^{1} \mathrm{H}$ NMR of compound $4 \mathrm{fa}\left(600 \mathrm{MHz}\right.$ in $\left.\mathrm{CDCl}_{3}\right)$

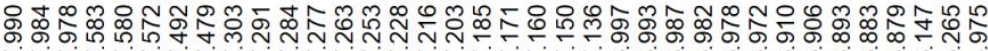

-Nan<smiles></smiles>

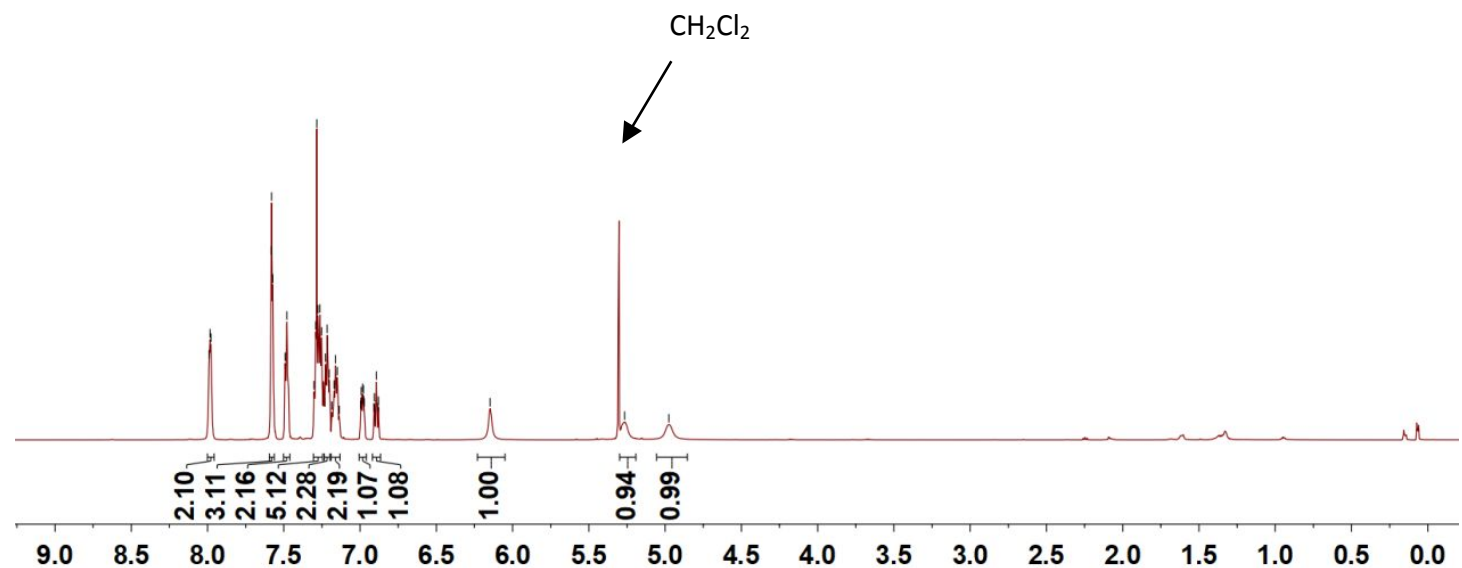

${ }^{13} \mathrm{C}$ NMR of compound $4 \mathrm{fa}\left(126 \mathrm{MHz}\right.$ in $\left.\mathrm{CDCl}_{3}\right)$

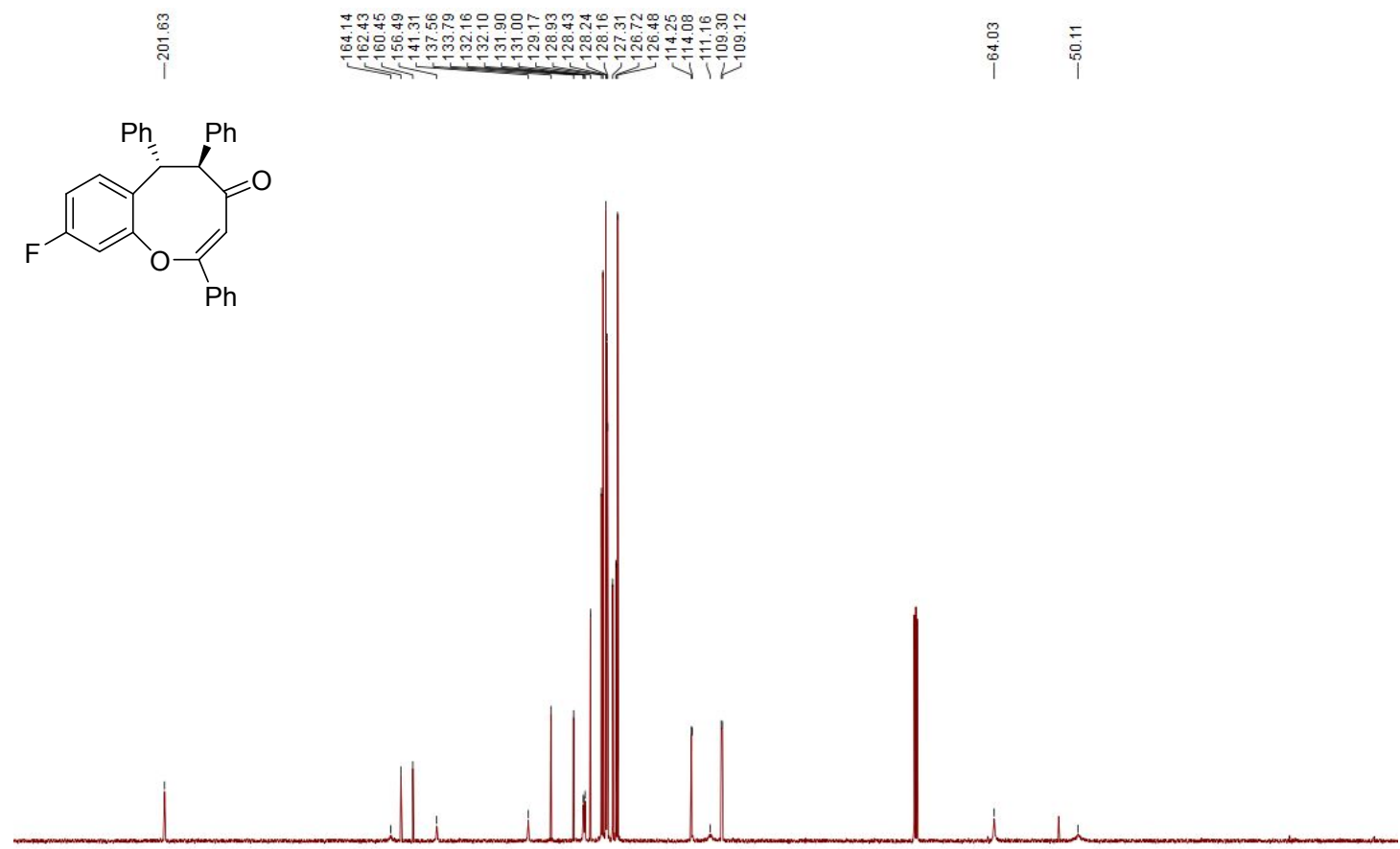

$\begin{array}{llllllllllllllllllllllllll}1 & 220 & 210 & 200 & 190 & 180 & 170 & 160 & 150 & 140 & 130 & 120 & 110 & 100 & 90 & 80 & 70 & 60 & 50 & 40 & 30 & 20 & 10 & 0\end{array}$ 
${ }^{1} \mathrm{H}$ NMR of compound $4 \mathrm{ga}\left(500 \mathrm{MHz}\right.$ in $\left.\mathrm{CDCl}_{3}\right)$

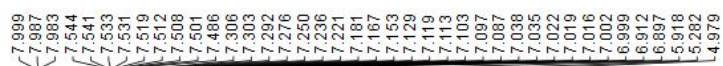<smiles>O=C1/C=C(\c2ccccc2)Oc2c(F)cccc2[C@H](c2ccccc2)C(=O)C1</smiles>

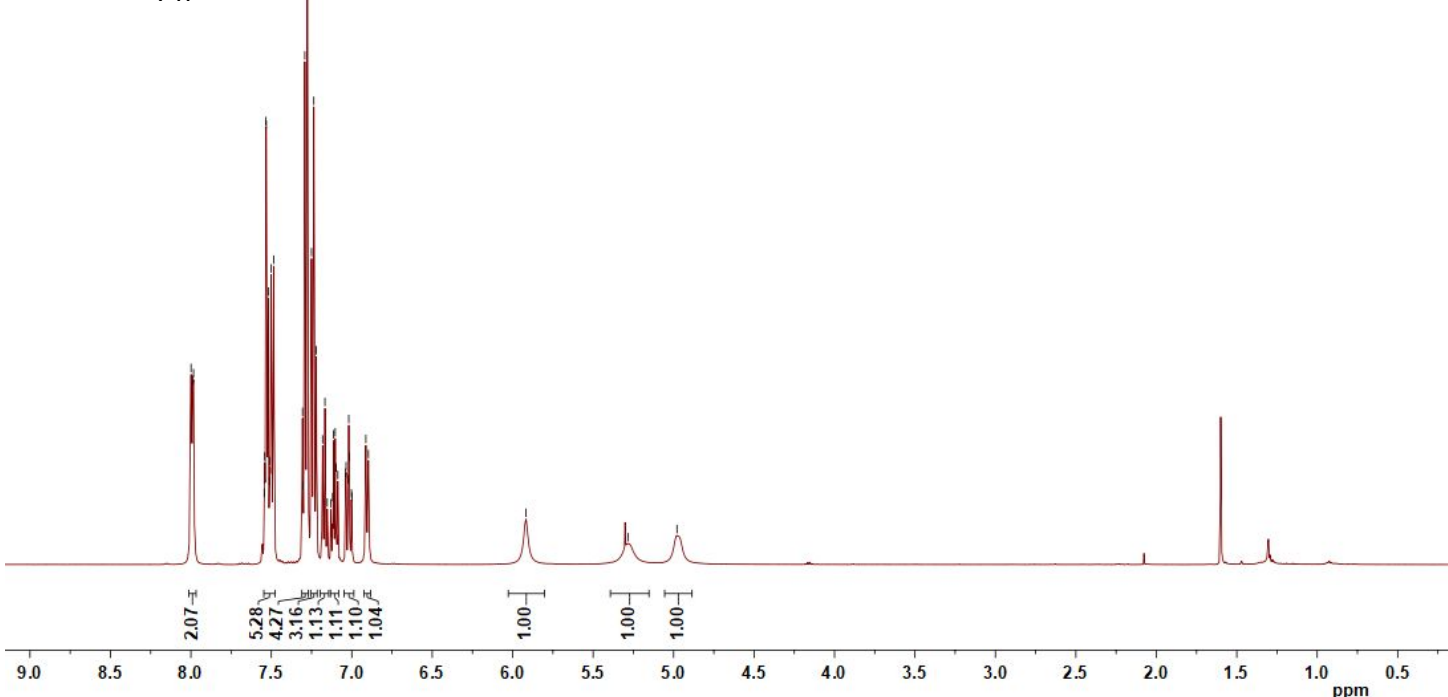

${ }^{13} \mathrm{C}$ NMR of compound $4 \mathrm{ga}\left(126 \mathrm{MHz}\right.$ in $\left.\mathrm{CDCl}_{3}\right)$

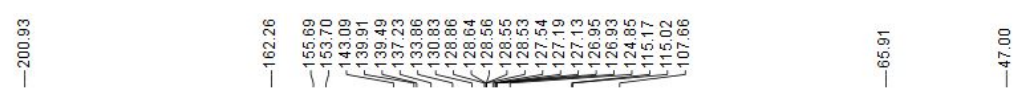<smiles>O=C1/C=C(\c2ccccc2)C(c2ccccc2)C(c2ccccc2)c2cccc(F)c2O1</smiles>

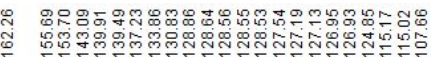

$\mathrm{Ph}$

\begin{tabular}{llllllllllllllllllllllllllllll}
\hline 220 & 210 & 200 & 190 & 180 & 170 & 160 & 150 & 140 & 130 & 120 & 110 & 100 & 90 & 80 & 70 & 60 & 50 & 40 & 30 & 20 & 10 & 0
\end{tabular} 
${ }^{1} \mathrm{H}$ NMR of compound 4ha (500 $\mathrm{MHz}$ in $\left.\mathrm{CDCl}_{3}\right)$

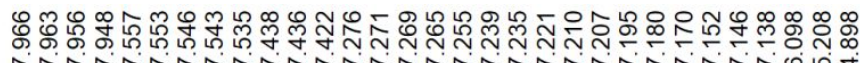

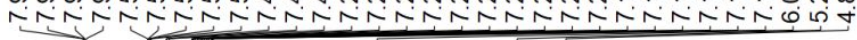<smiles>O=C1C=C(c2ccccc2)Oc2ccc(Cl)cc2[C@@H](c2ccccc2)C1c1ccccc1</smiles>

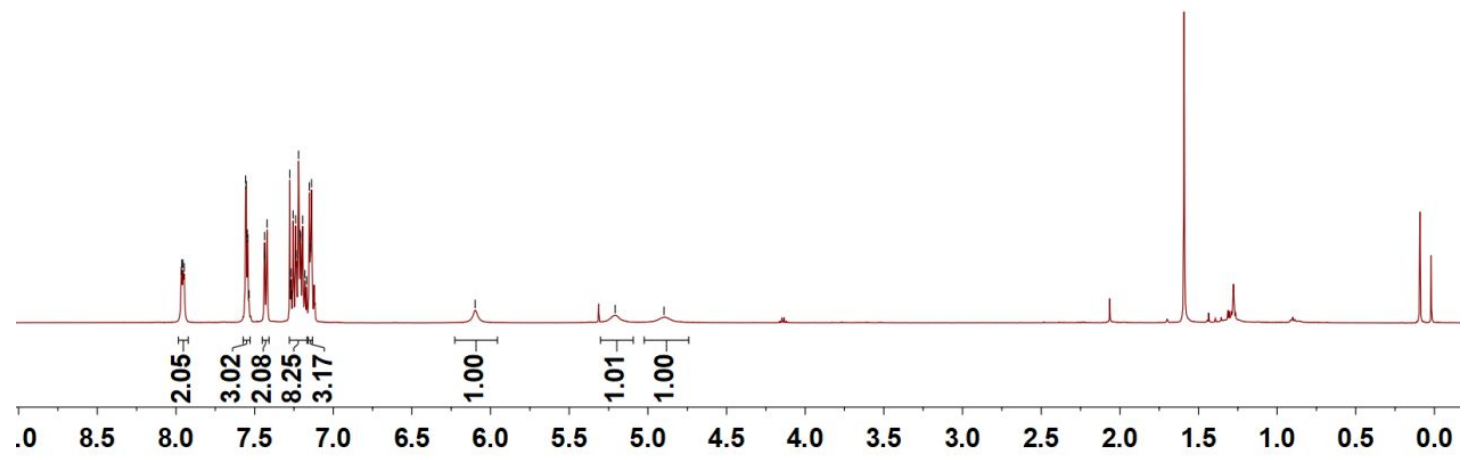

${ }^{13} \mathrm{C}$ NMR of compound 4ha (126 $\mathrm{MHz}$ in $\left.\mathrm{CDCl}_{3}\right)$<smiles>O=C1C=C(c2ccccc2)C(c2ccccc2)C(c2ccccc2)c2cc(Cl)ccc2O1</smiles>

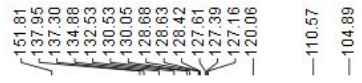

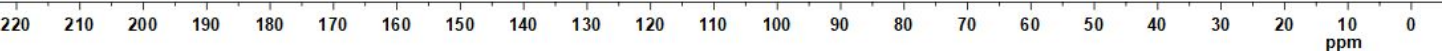


${ }^{1} \mathrm{H}$ NMR of compound 4 ia (500 $\mathrm{MHz}$ in $\left.\mathrm{CDCl}_{3}\right)$

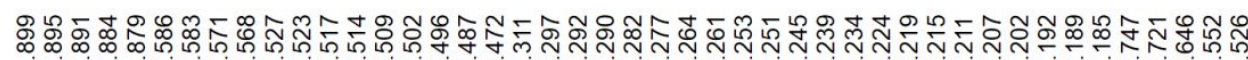

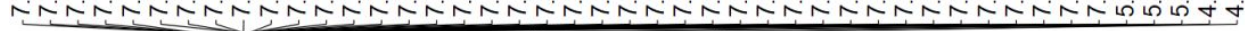<smiles>O=C1C=C(c2ccccc2)C(c2ccccc2)c2cccc(C(F)(F)F)c2O1</smiles>

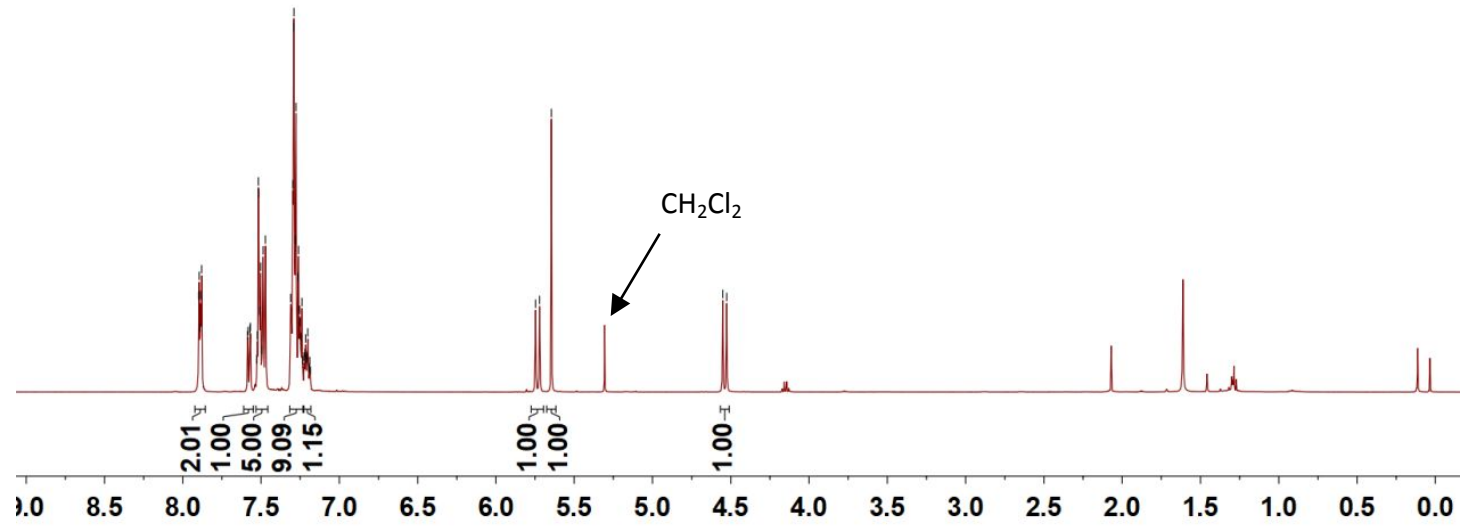

${ }^{13} \mathrm{C}$ NMR of compound 4 ia (126 $\mathrm{MHz}$ in $\mathrm{CDCl}_{3}$ )<smiles>O=C1C=C(c2ccccc2)[C@H](c2ccccc2)[C@H](c2ccccc2)c2ccccc2O1</smiles>

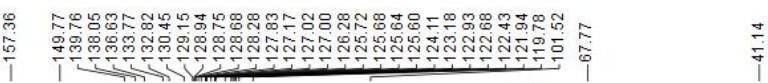
\begin{tabular}{llllllllllllllllllllllllllllllll}
\hline & 220 & 210 & 200 & 190 & 180 & 170 & 160 & 150 & 140 & 130 & 120 & 110 & 100 & 90 & 80 & 70 & 60 & 50 & 40 & 30 & 20 & 10 & 0
\end{tabular} 
${ }^{1} \mathrm{H}$ NMR of compound $4 \mathrm{ja}\left(500 \mathrm{MHz}\right.$ in $\left.\mathrm{CDCl}_{3}\right)$

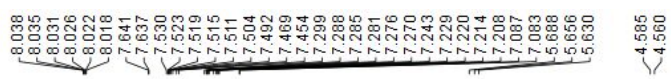<smiles>O=C1/C=C(\c2ccccc2)C(c2ccccc2)[C@@H](c2ccccc2)c2cc(Br)cc(Br)c2OC1=O</smiles>

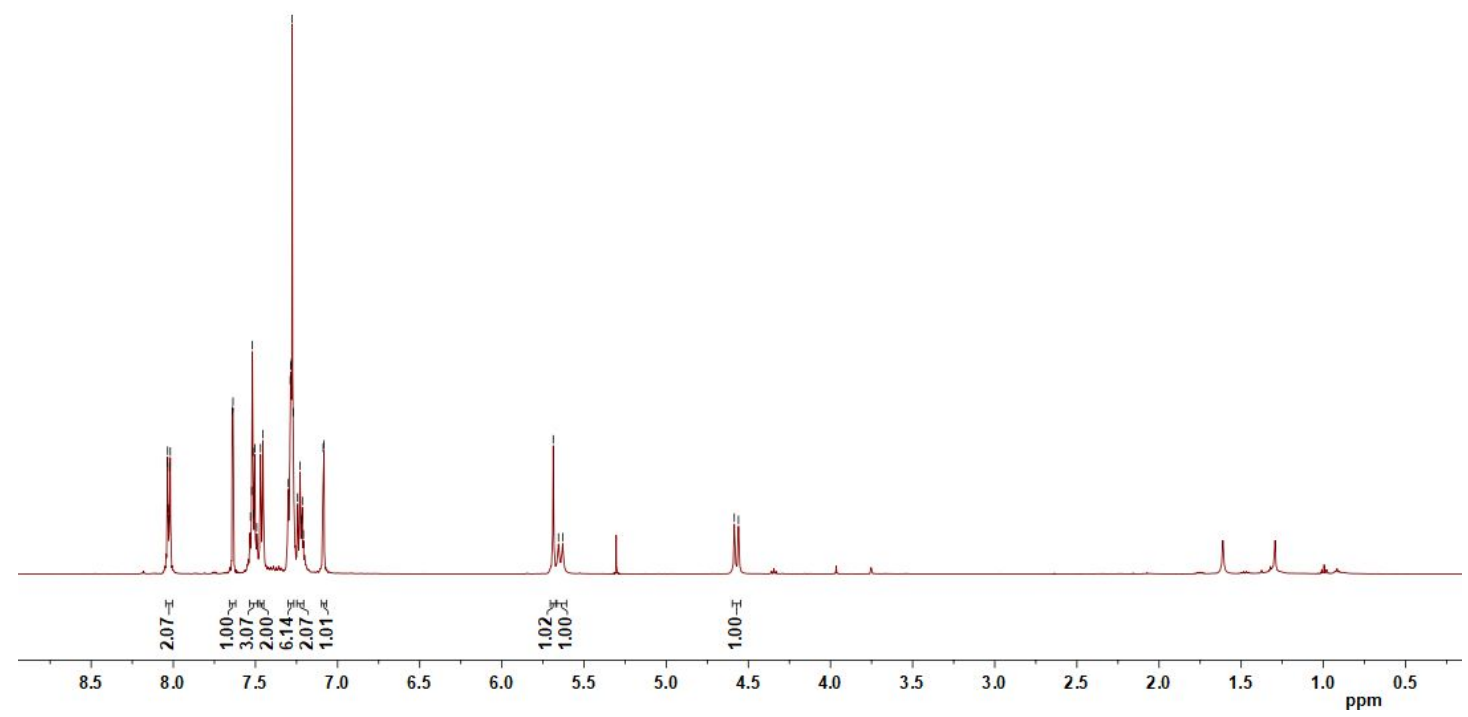

${ }^{13} \mathrm{C}$ NMR of compound $4 \mathrm{ja}\left(126 \mathrm{MHz}\right.$ in $\left.\mathrm{CDCl}_{3}\right)$

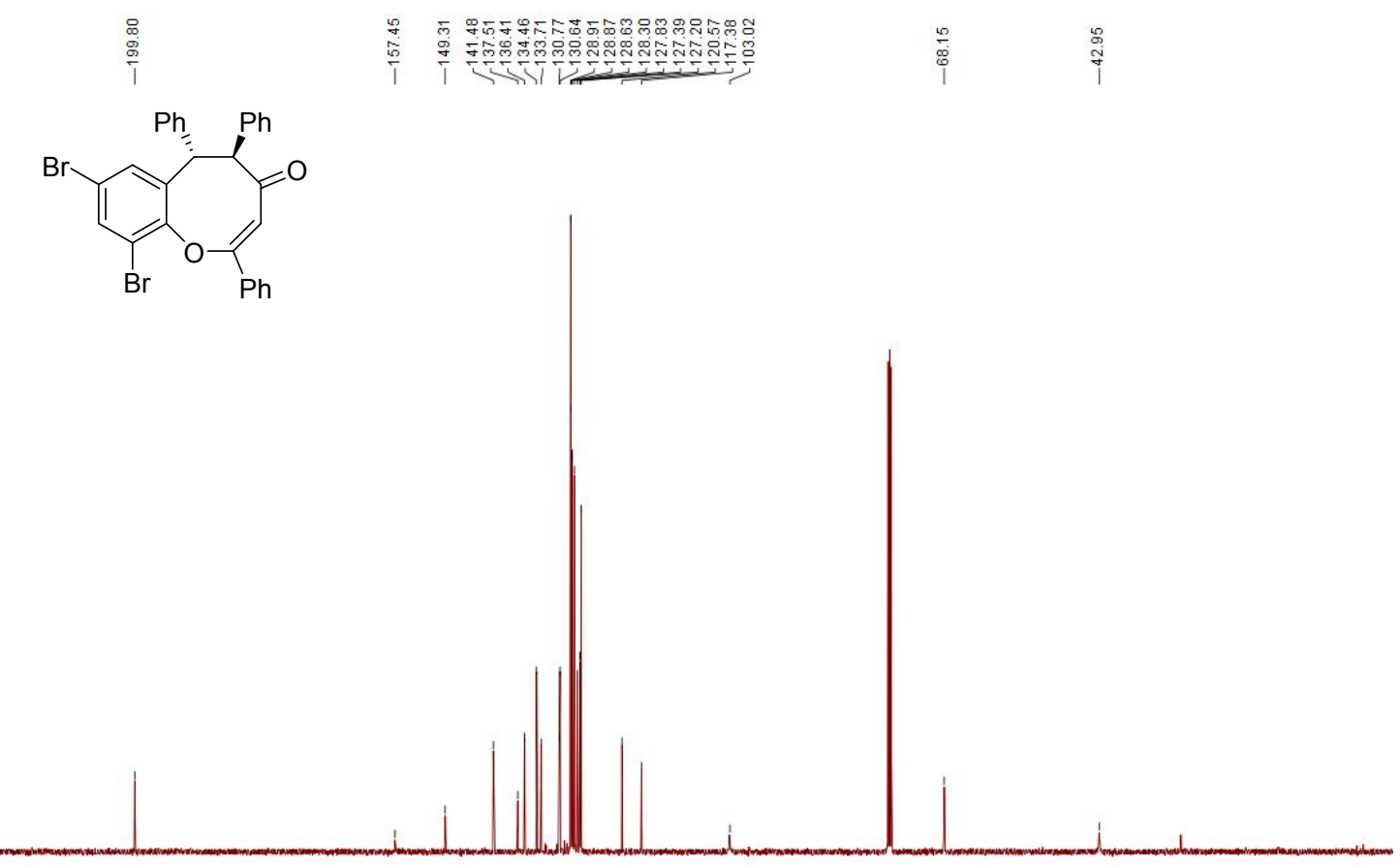

\begin{tabular}{llllllllllllllllllllllllllll}
\hline & 210 & 200 & 190 & 180 & 170 & 160 & 150 & 140 & 130 & 120 & 110 & 100 & 90 & 80 & 70 & 60 & 50 & 40 & 30 & 20 & 10 & 0 \\
$\mathrm{ppm}$ &
\end{tabular} 
${ }^{1} \mathrm{H}$ NMR of compound $4 \mathrm{ka} \mathrm{(500} \mathrm{MHz}$ in $\left.\mathrm{CDCl}_{3}\right)$

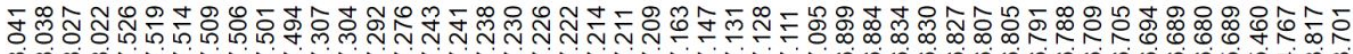

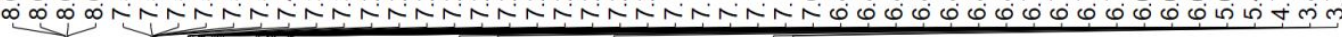<smiles>COc1cccc(C2c3cccc(OC)c3OC(c3ccccc3)CC(=O)C2c2ccccc2)c1</smiles>
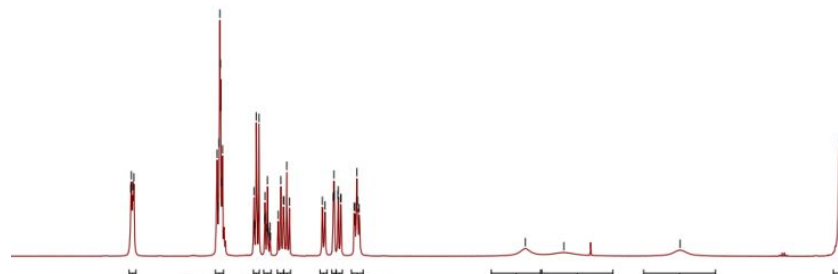

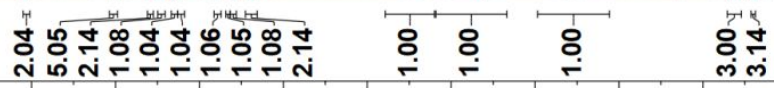

$\begin{array}{lllllllllllllllll}8.5 & 8.0 & 7.5 & 7.0 & 6.5 & 6.0 & 5.5 & 5.0 & 4.5 & 4.0 & 3.5 & 3.0 & 2.5 & 2.0 & 1.5 & 1.0 & 0.5\end{array}$

${ }^{13} \mathrm{C}$ NMR of compound $4 \mathrm{ka}\left(126 \mathrm{MHz}\right.$ in $\left.\mathrm{CDCl}_{3}\right)$<smiles>COc1cccc([C@H]2c3cccc(OC)c3O/C(c3ccccc3)=C\C(=O)C(c3ccccc3)[C@H]2c2ccccc2)c1</smiles>

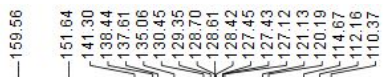

$\begin{array}{lllllllllllll}220 & 210 & 200 & 190 & 180 & 170 & 160 & 150 & 140 & 130 & 120 & 1\end{array}$ 
${ }^{1} \mathrm{H}$ NMR of compound 4la (500 $\mathrm{MHz}$ in $\left.\mathrm{CDCl}_{3}\right)$

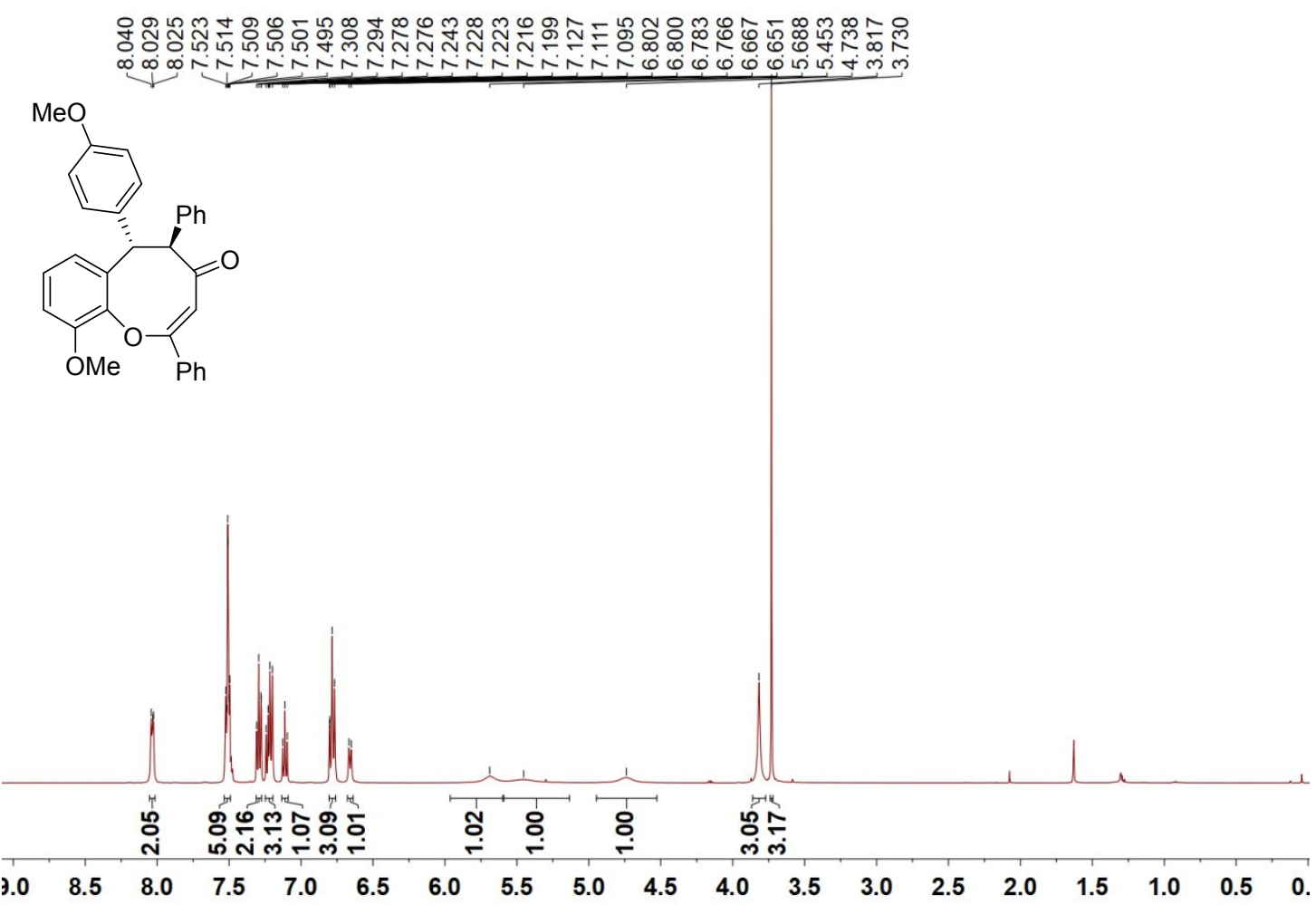

${ }^{13} \mathrm{C}$ NMR of compound $4 \mathrm{la}\left(126 \mathrm{MHz}\right.$ in $\left.\mathrm{CDCl}_{3}\right)$
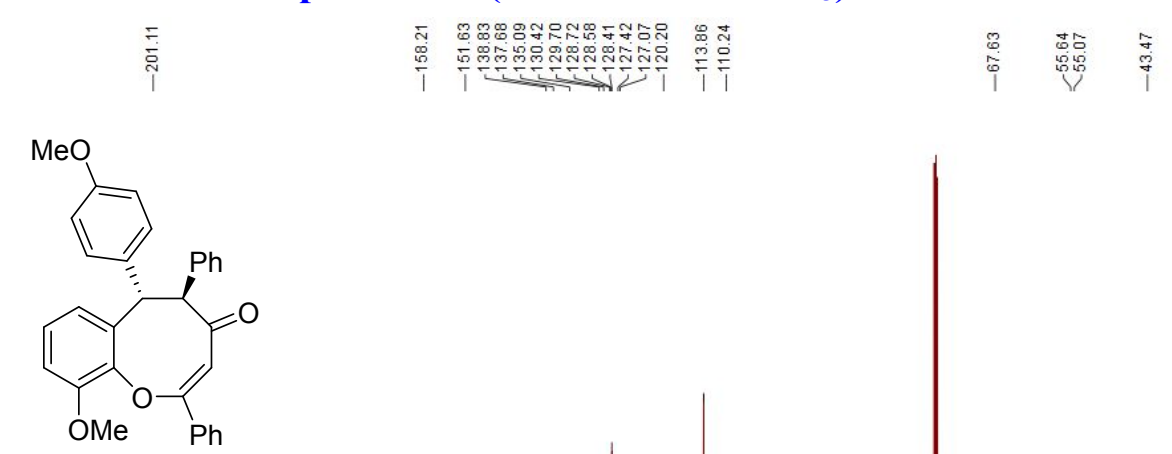

\begin{tabular}{llllllllllllllllllllllllll}
\hline 1 & 210 & 200 & 190 & 180 & 170 & 160 & 150 & 140 & 130 & 120 & 110 & 100 & 90 & 80 & 70 & 60 & 50 & 40 & 30 & 20 & 10 & 0 \\
$\mathrm{ppm}$ &
\end{tabular} 
${ }^{1} \mathrm{H}$ NMR of compound $4 \mathrm{ma}\left(500 \mathrm{MHz}\right.$ in $\mathrm{CDCl}_{3}$ )
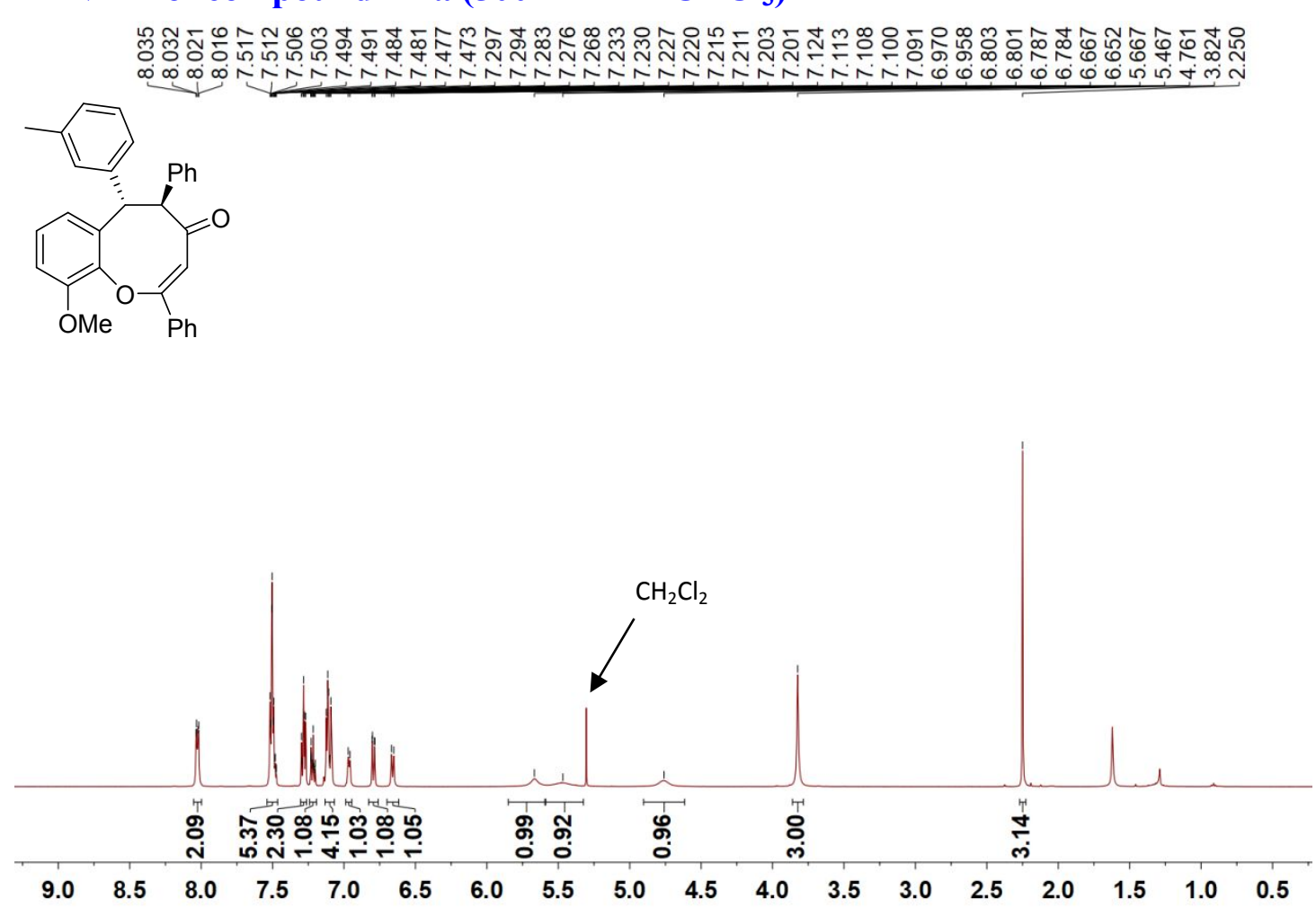

${ }^{13} \mathrm{C}$ NMR of compound $4 \mathrm{ma}\left(126 \mathrm{MHz}\right.$ in $\left.\mathrm{CDCl}_{3}\right)$

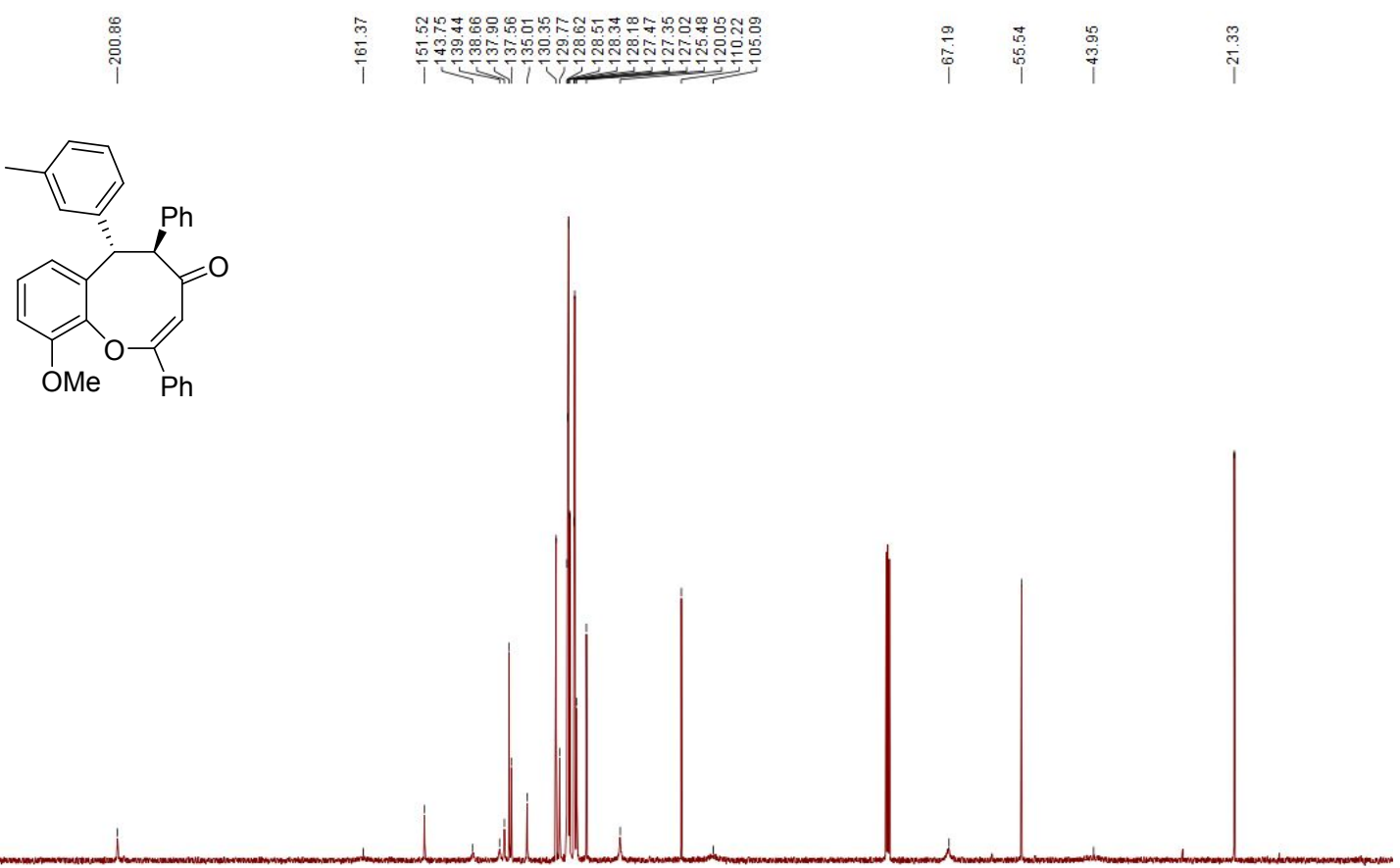

\begin{tabular}{rllllllllllllllllllllllllll}
\hline 220 & 210 & 200 & 190 & 180 & 170 & 160 & 150 & 140 & 130 & 120 & 110 & 100 & 90 & 80 & 70 & 60 & 50 & 40 & 30 & 20 & 10 & 0
\end{tabular} 
${ }^{1} \mathrm{H}$ NMR of compound 4na (500 $\mathrm{MHz}$ in $\left.\mathrm{CDCl}_{3}\right)$

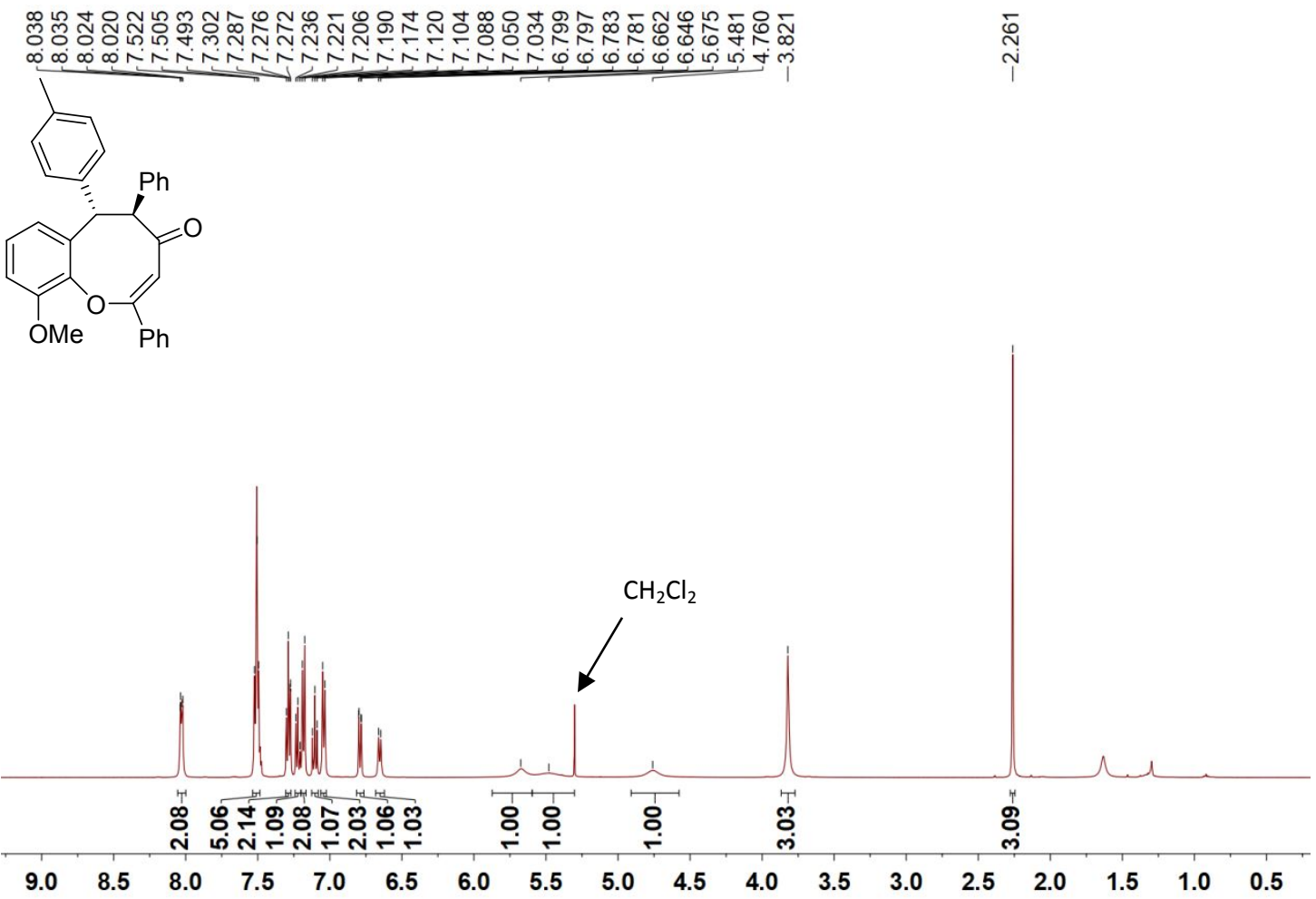

${ }^{13} \mathrm{C}$ NMR of compound 4na (126 $\mathrm{MHz}$ in $\left.\mathrm{CDCl}_{3}\right)$

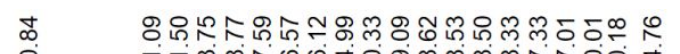

ì

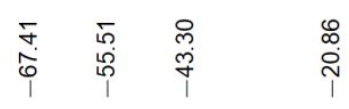

OMe

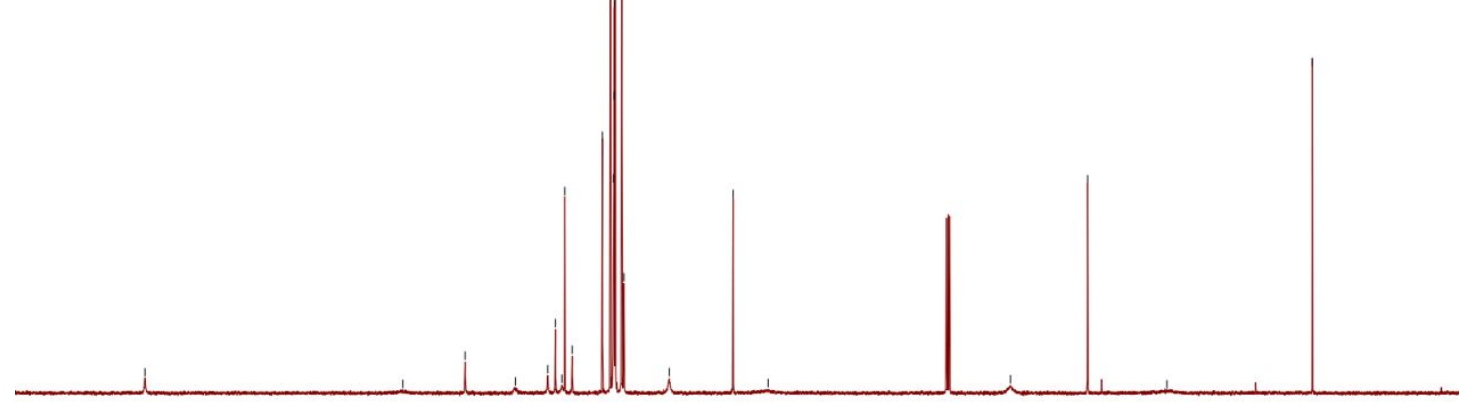

$\begin{array}{llllllllllllllllllllllll}20 & 210 & 200 & 190 & 180 & 170 & 160 & 150 & 140 & 130 & 120 & 110 & 100 & 90 & 80 & 70 & 60 & 50 & 40 & 30 & 20 & 10 & 0\end{array}$ 
${ }^{1} \mathrm{H}$ NMR of compound $40 a\left(500 \mathrm{MHz}\right.$ in $\left.\mathrm{CDCl}_{3}\right)$

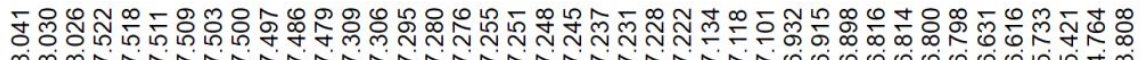<smiles>COc1cccc2c1OC(c1ccccc1)C(c1ccccc1)C(=O)C=C2c1ccccc1</smiles>

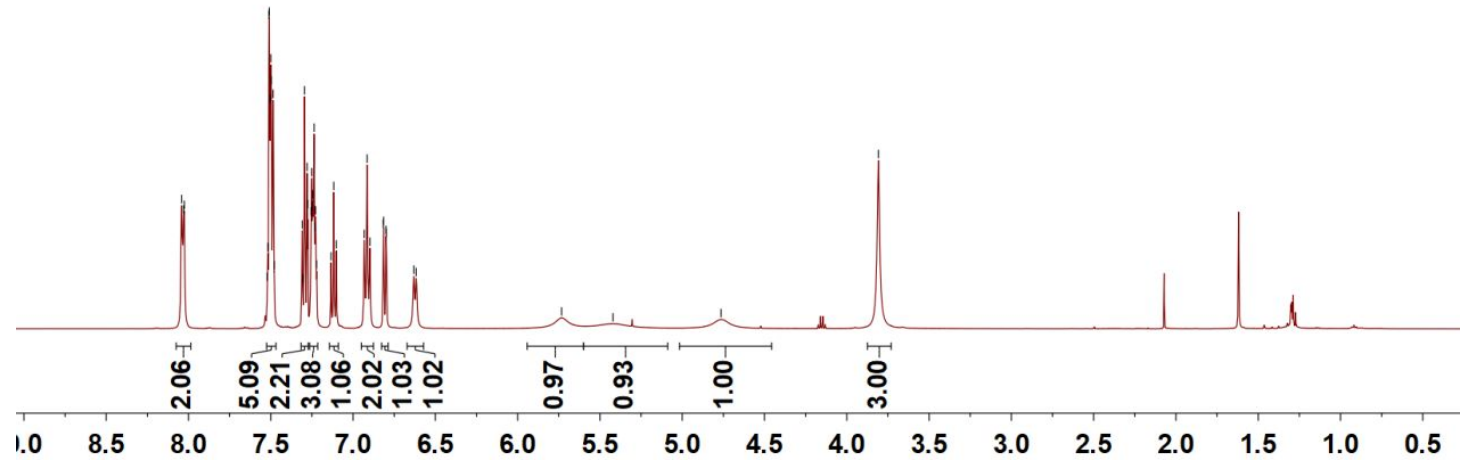

${ }^{13} \mathrm{C}$ NMR of compound $40 a\left(126 \mathrm{MHz}\right.$ in $\left.\mathrm{CDCl}_{3}\right)$

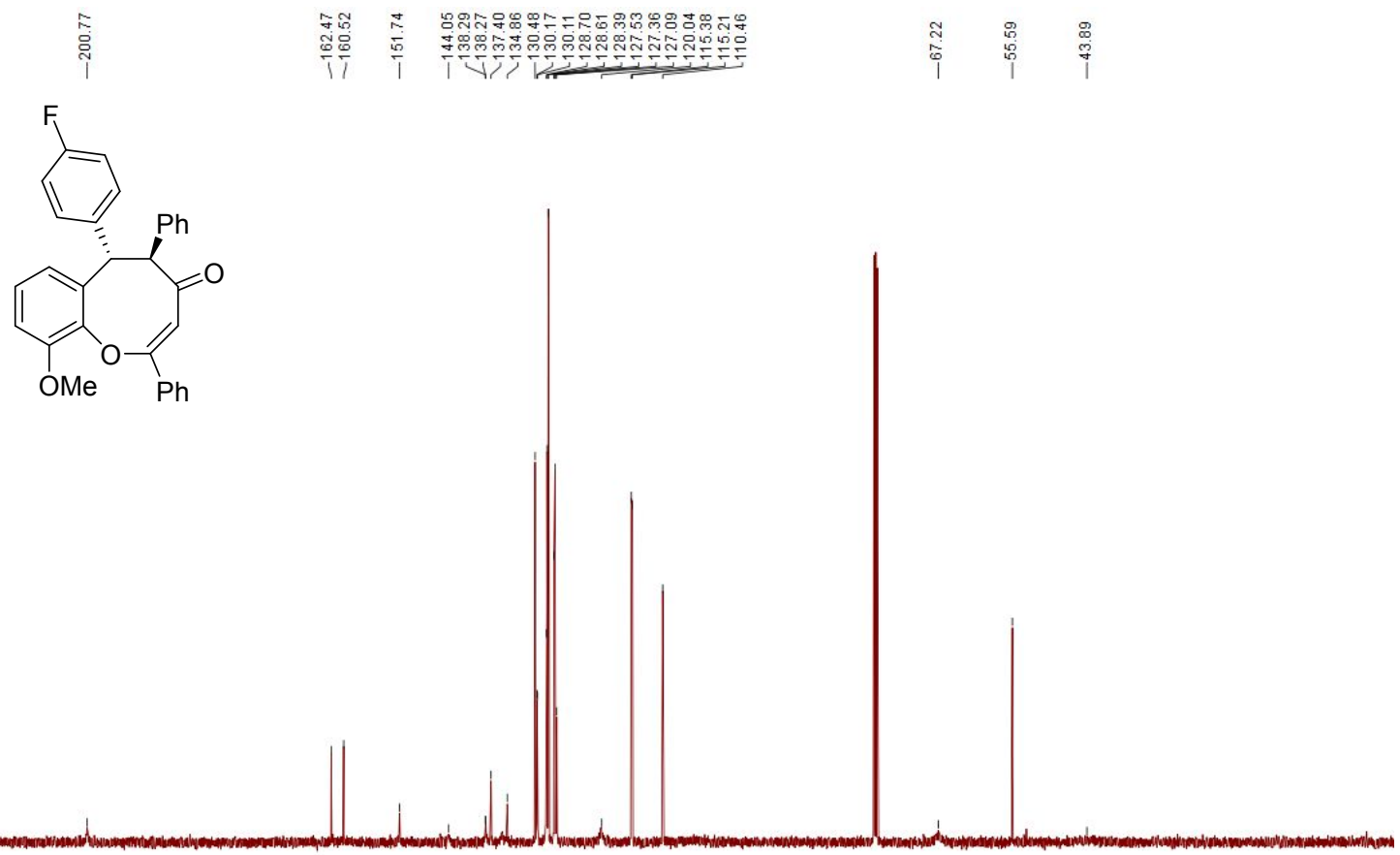

$\begin{array}{rllllllllllllllllllllllll}210 & 200 & 190 & 180 & 170 & 160 & 150 & 140 & 130 & 120 & 110 & 100 & 90 & 80 & 70 & 60 & 50 & 40 & 30 & 20 & 10 & 0\end{array}$ 
${ }^{1} \mathrm{H}$ NMR of compound 4pa (500 $\mathrm{MHz}$ in $\left.\mathrm{CDCl}_{3}\right)$

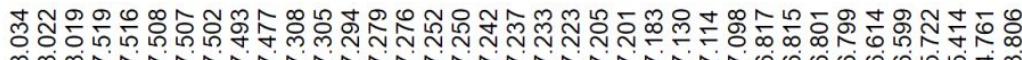

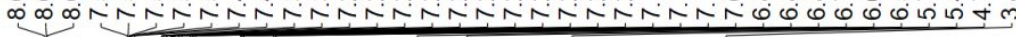<smiles></smiles>

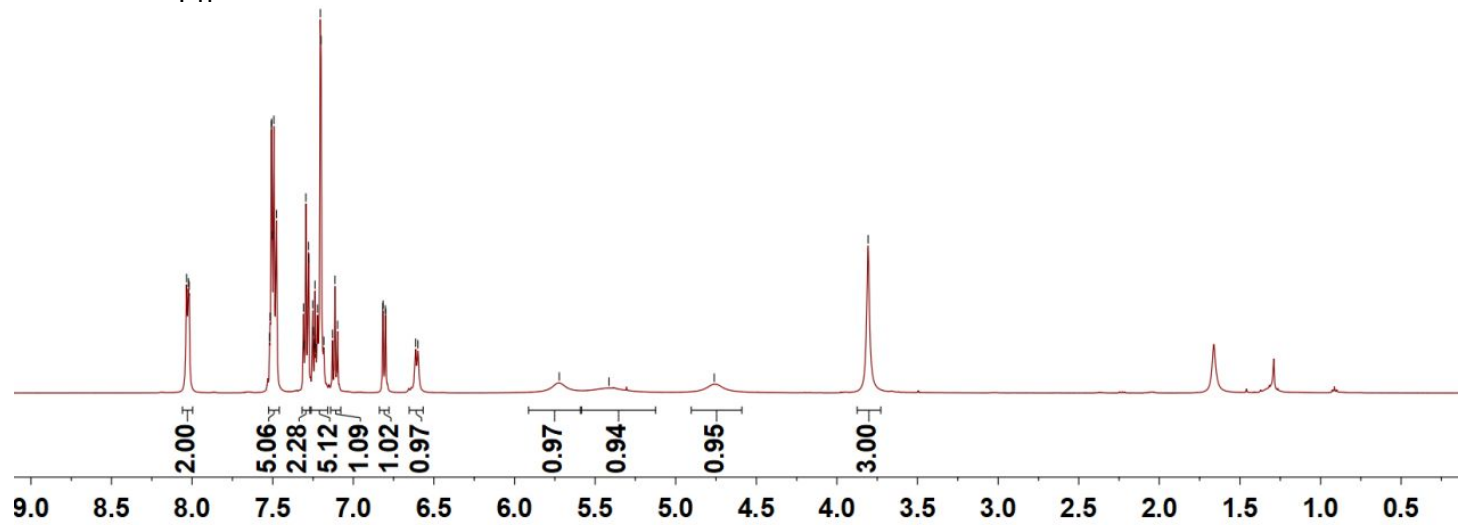

${ }^{13} \mathrm{C}$ NMR of compound 4pa (126 $\mathrm{MHz}$ in $\mathrm{CDCl}_{3}$ )

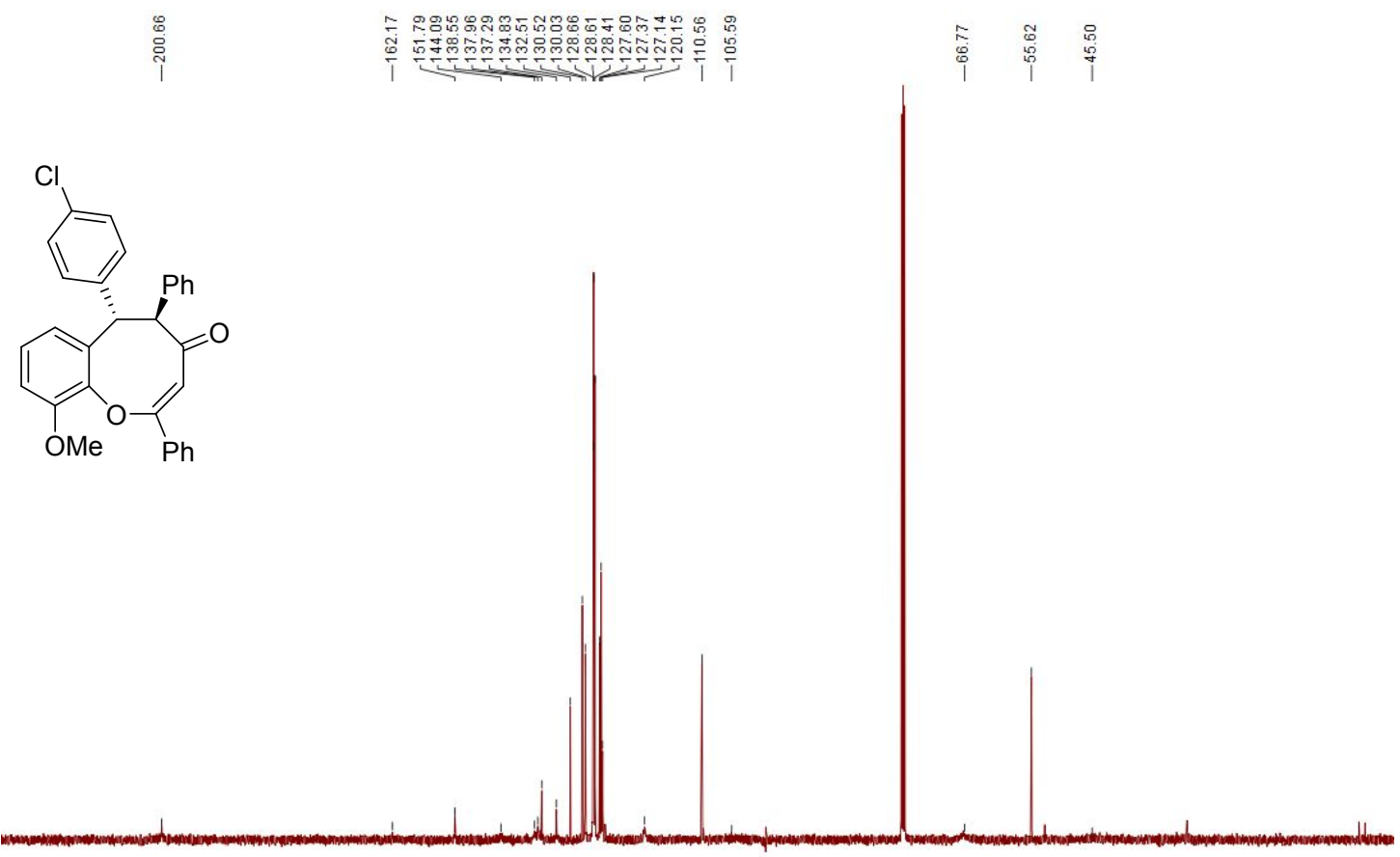

$\begin{array}{llllllllllllllllllllllllll}220 & 210 & 200 & 190 & 180 & 170 & 160 & 150 & 140 & 130 & 120 & 110 & 100 & 90 & 80 & 70 & 60 & 50 & 40 & 30 & 20 & 10 & 0 \\ \mathrm{ppm} & \end{array}$ 
${ }^{1} \mathrm{H}$ NMR of compound $4 \mathrm{qa}\left(500 \mathrm{MHz}\right.$ in $\left.\mathrm{CDCl}_{3}\right)$

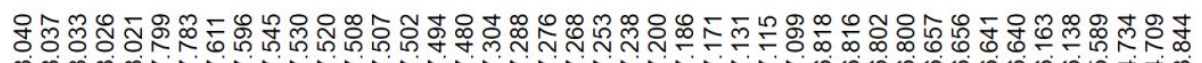

等<smiles>COc1cccc(C(c2ccccc2)c2ccccc2)c1OC1Oc2ccccc21</smiles>

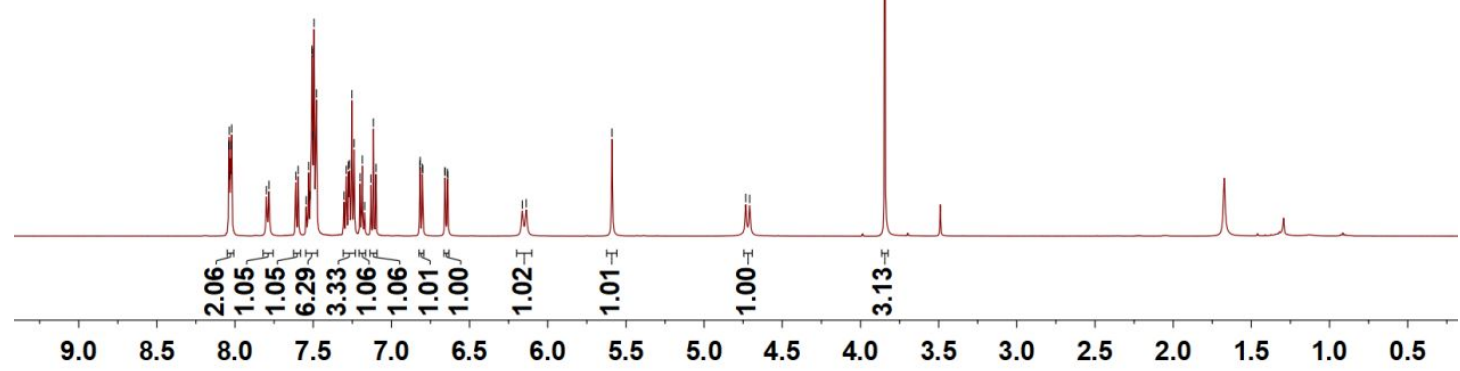

${ }^{13} \mathrm{C}$ NMR of compound 4qa (126 $\mathrm{MHz}$ in $\left.\mathrm{CDCl}_{3}\right)$

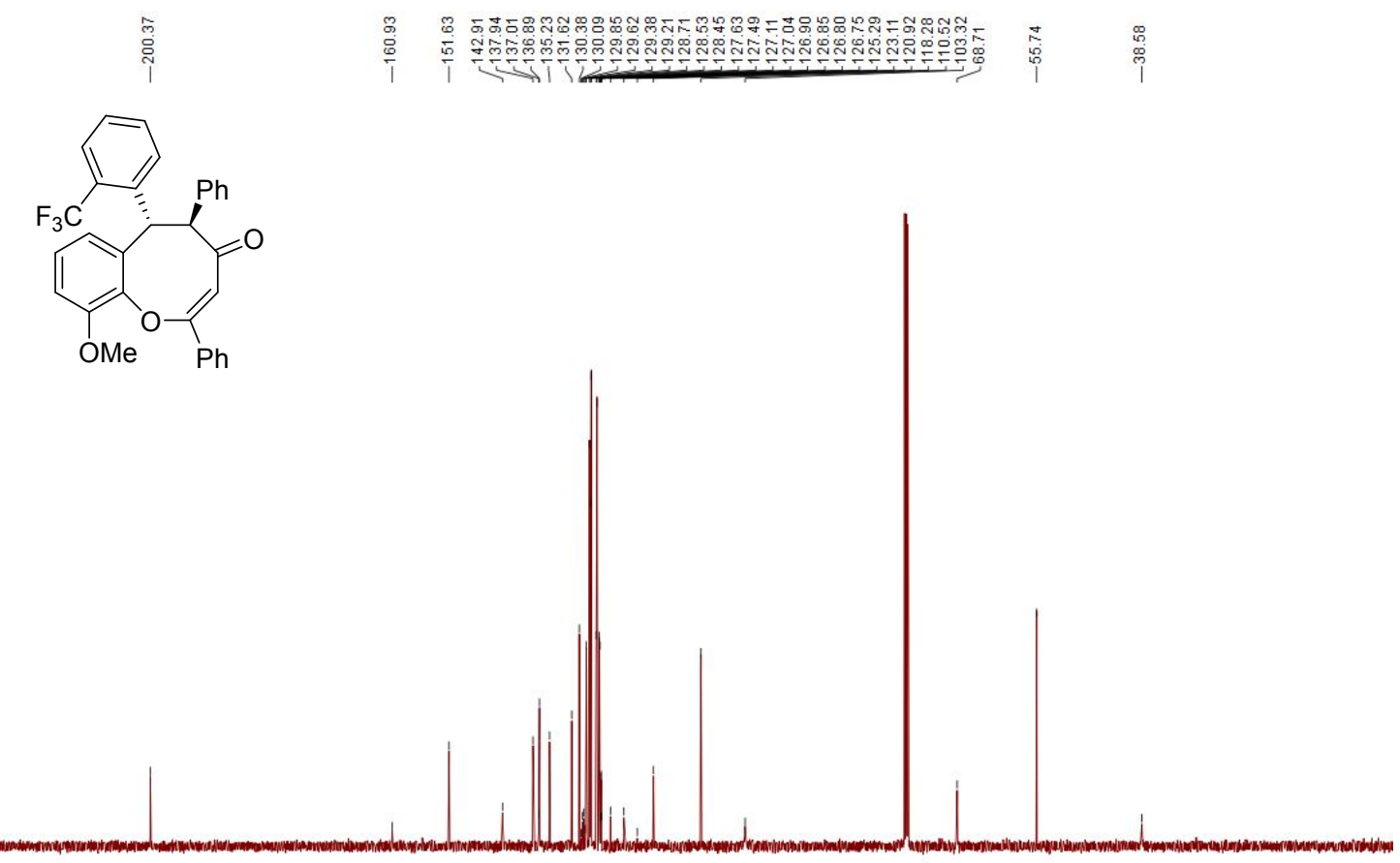

$\begin{array}{lllllllllllllllllllllllllllllll}220 & 210 & 200 & 190 & 180 & 170 & 160 & 150 & 140 & 130 & 120 & 110 & 100 & 90 & 80 & 70 & 60 & 50 & 40 & 30 & 20 & 10 & 0\end{array}$ 
${ }^{1} \mathrm{H}$ NMR of compound 4ra (500 $\mathrm{MHz}$ in $\left.\mathrm{CDCl}_{3}\right)$

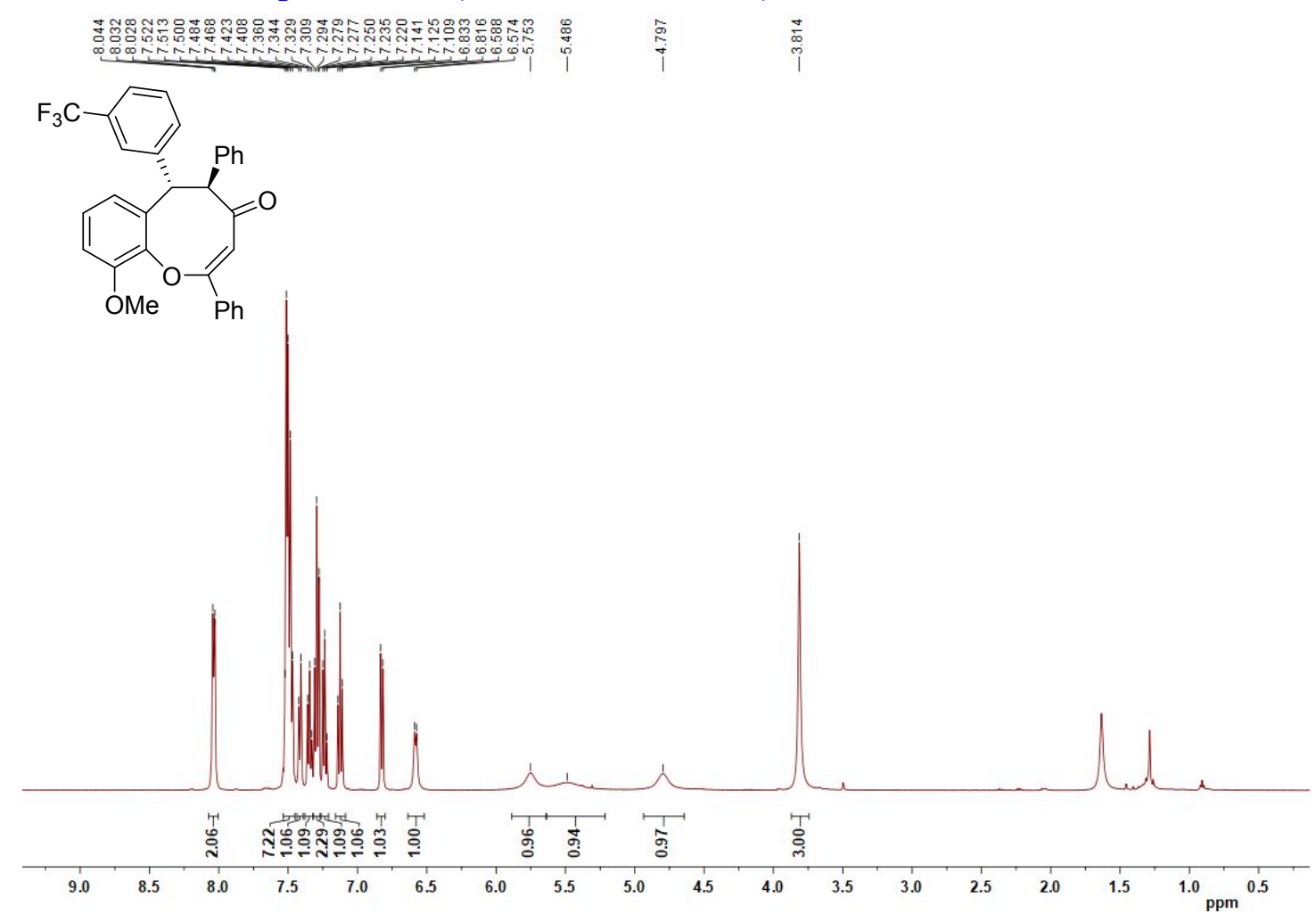

${ }^{13} \mathrm{C}$ NMR of compound 4ra (126 $\mathrm{MHz}$ in $\left.\mathrm{CDCl}_{3}\right)$

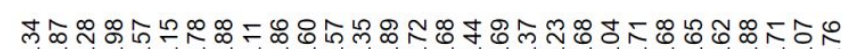

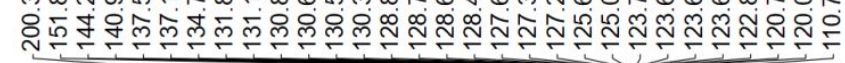<smiles>COc1cccc2c1OC(c1ccccc1)C=C(C(=O)c1ccccc1)C2c1ccccc1</smiles>

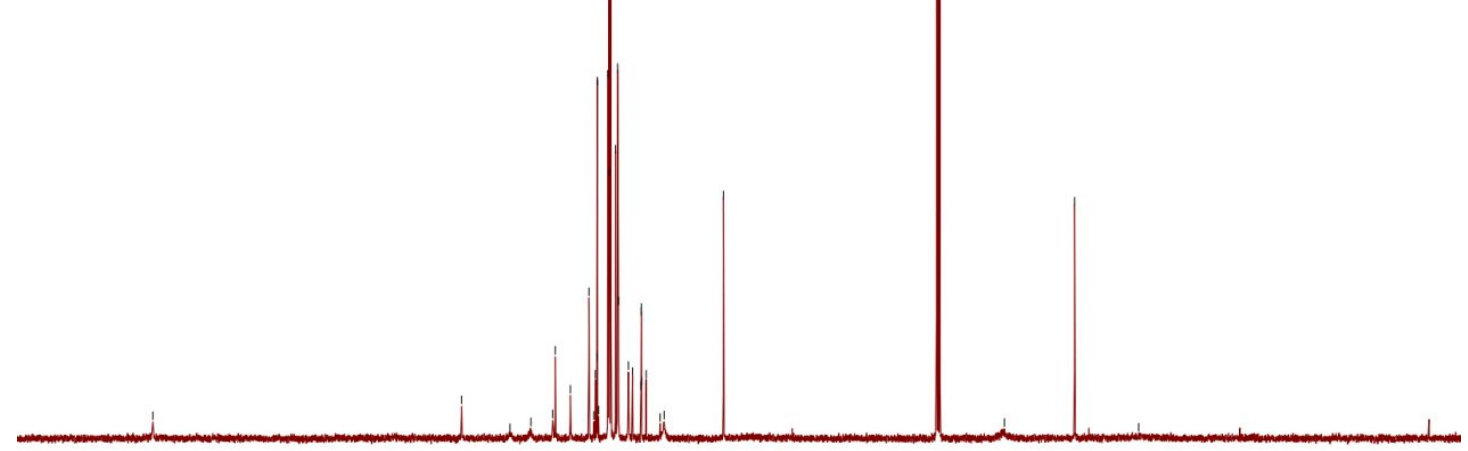

$\begin{array}{lllllllllllllllllllllll}20 & 210 & 200 & 190 & 180 & 170 & 160 & 150 & 140 & 130 & 120 & 110 & 100 & 90 & 80 & 70 & 60 & 50 & 40 & 30 & 20 & 10 & 0\end{array}$ 
${ }^{1} \mathrm{H}$ NMR of compound 4sa (500 $\mathrm{MHz}$ in $\mathrm{CDCl}_{3}$ )

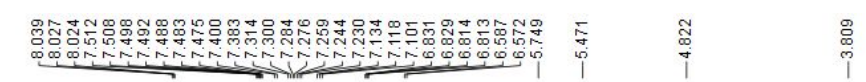<smiles>COc1cccc2c1OC(c1ccccc1)C(c1ccc(C(F)(F)F)cc1)C(=O)/C=C\2c1ccccc1</smiles>

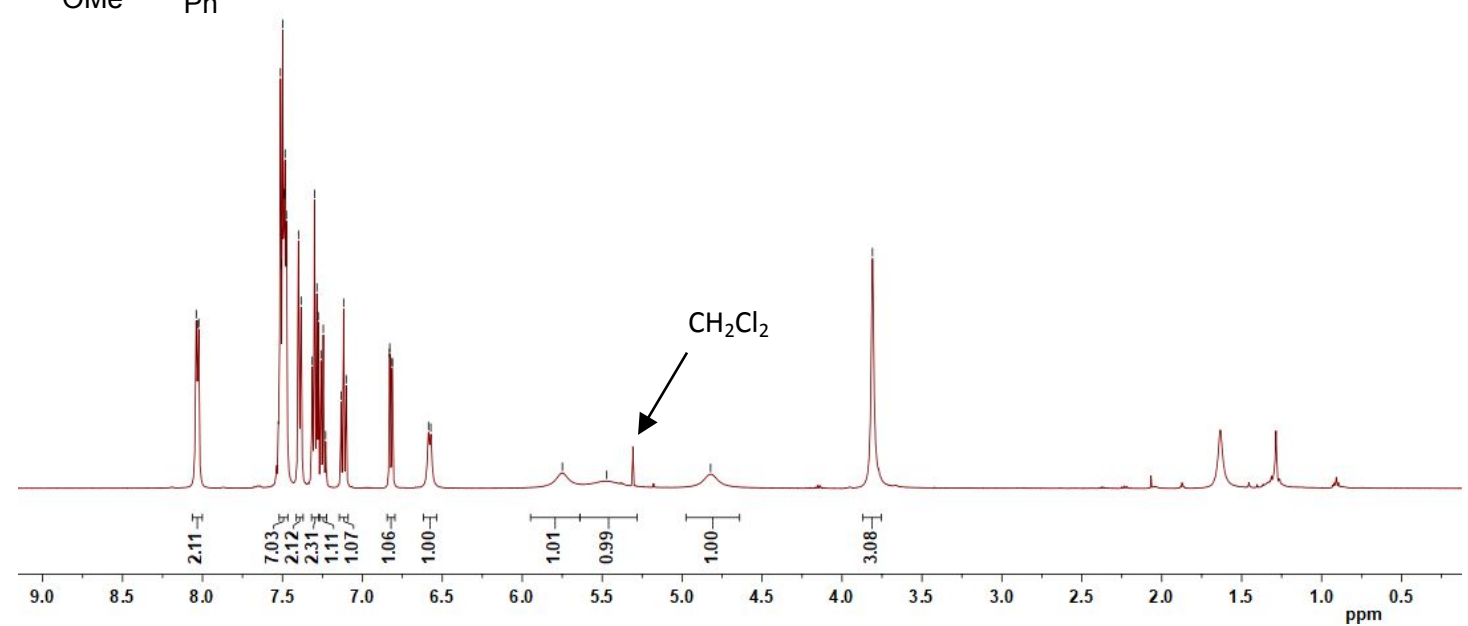

${ }^{13} \mathrm{C}$ NMR of compound 4sa (126 $\mathrm{MHz}$ in $\mathrm{CDCl}_{3}$ )

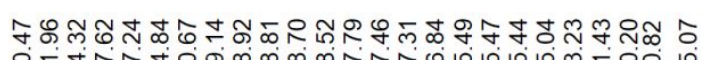

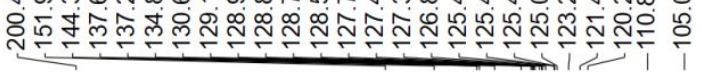<smiles>COc1cccc2c1OC(c1ccccc1)C(c1ccc(C(F)(F)F)cc1)C(=O)/C=C\2c1ccccc1</smiles> 
${ }^{1} \mathrm{H}$ NMR of compound 4 ta $\left(500 \mathrm{MHz}\right.$ in $\left.\mathrm{CDCl}_{3}\right)$

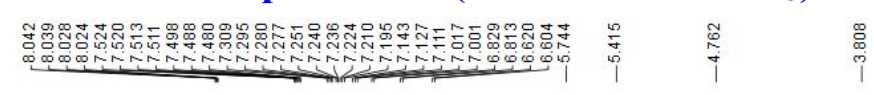<smiles>COc1cccc2c1OC(c1ccccc1)CC(=O)C(c1ccccc1)C2c1cccc(OC(F)(F)F)c1</smiles>

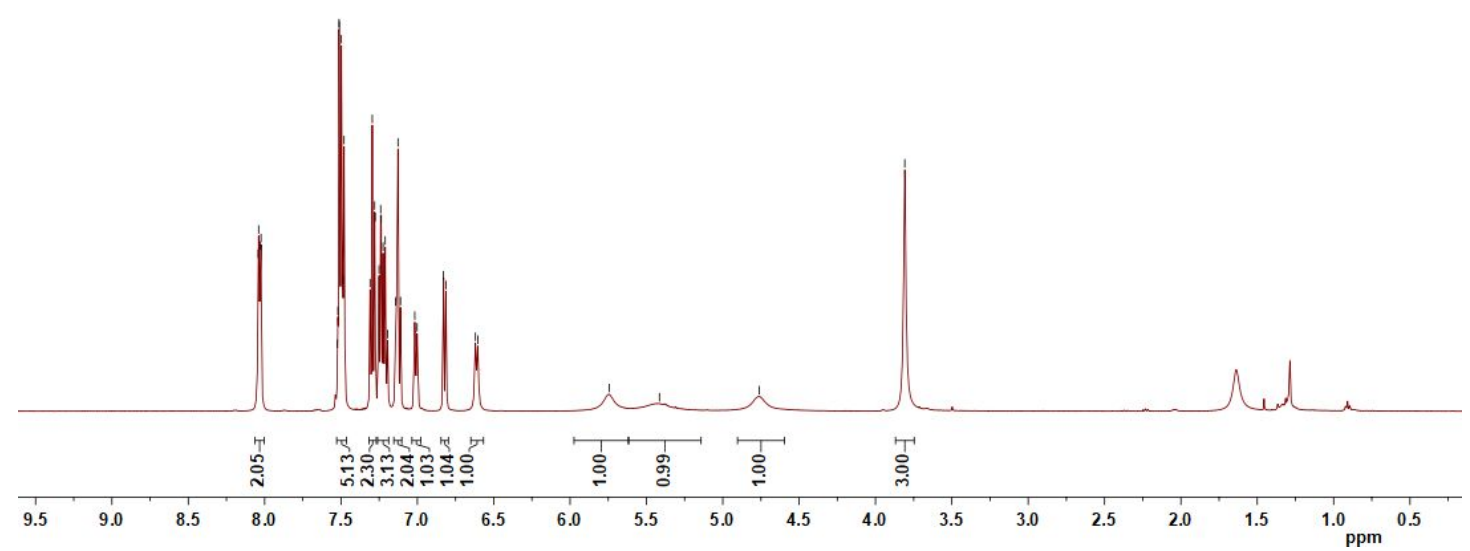

${ }^{13} \mathrm{C}$ NMR of compound 4ta (126 $\mathrm{MHz}$ in $\mathrm{CDCl}_{3}$ )

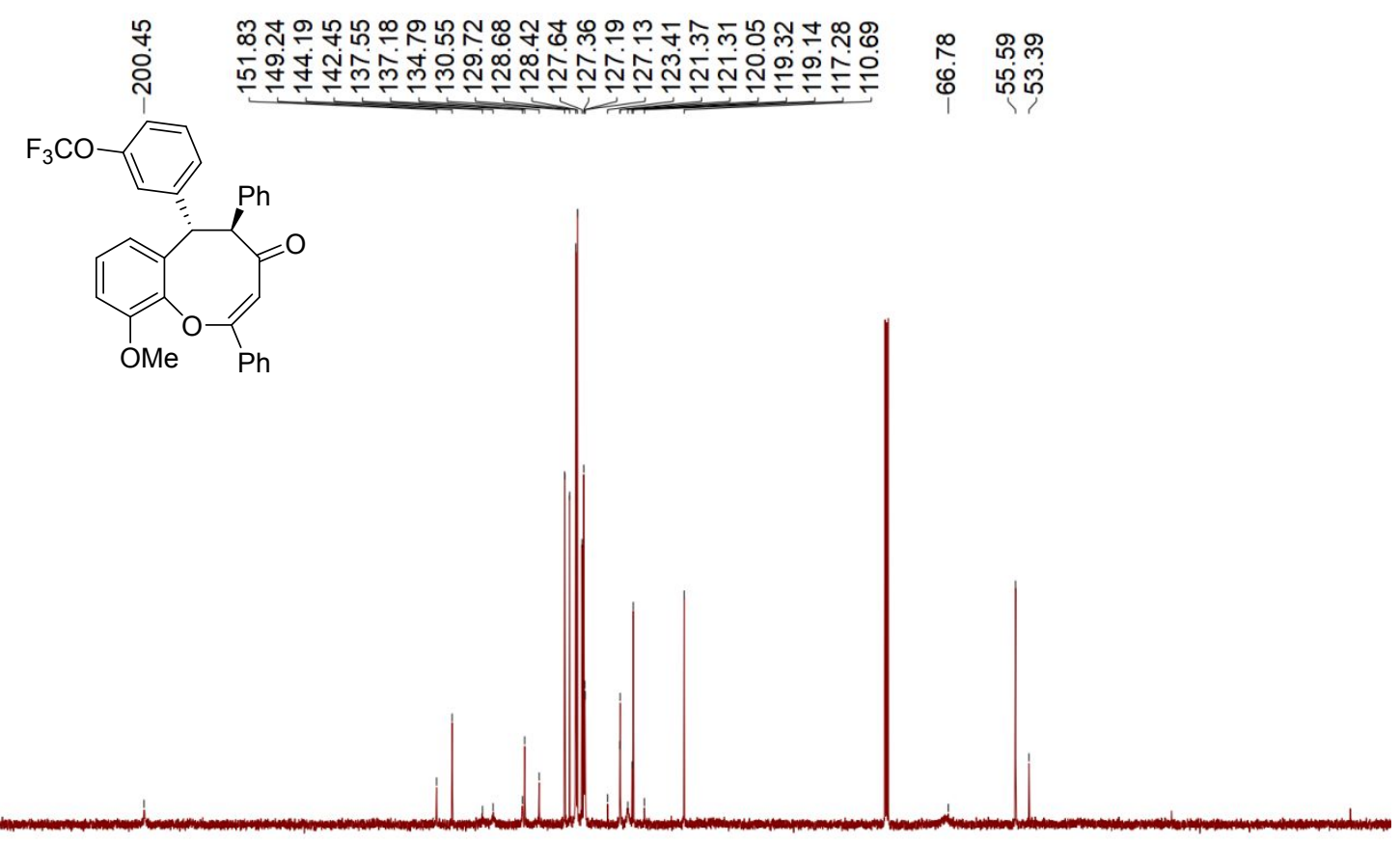

$\begin{array}{lllllllllllllllllllllll}220 & 210 & 200 & 190 & 180 & 170 & 160 & 150 & 140 & 130 & 120 & 110 & 100 & 90 & 80 & 70 & 60 & 50 & 40 & 30 & 20 & 10 & 0\end{array}$ 
${ }^{1} \mathrm{H}$ NMR of compound 4ua (500 $\mathrm{MHz}$ in $\mathrm{CDCl}_{3}$ )

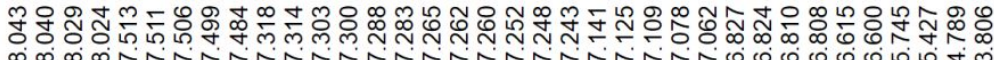

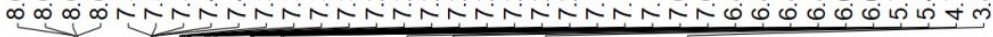<smiles>COc1cccc2c1Oc1ccccc1[C@H](c1ccc(OC(F)(F)F)cc1)C(=O)/C=C\2O</smiles>

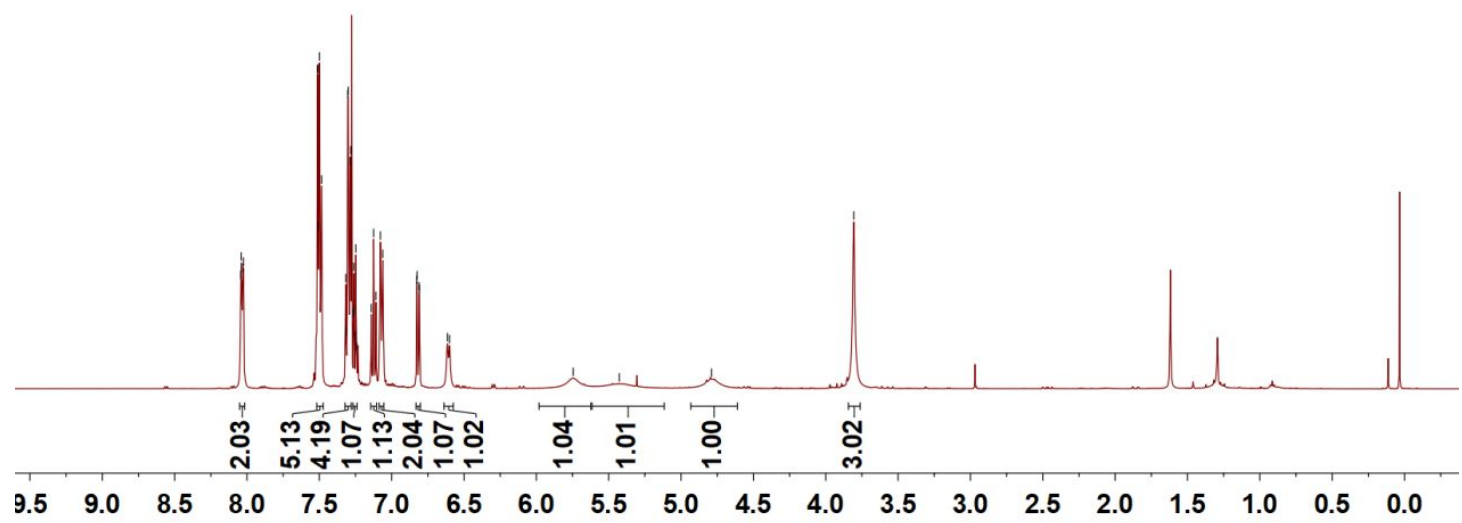

${ }^{13} \mathrm{C}$ NMR of compound 4ua (126 $\mathrm{MHz}$ in $\mathrm{CDCl}_{3}$ )

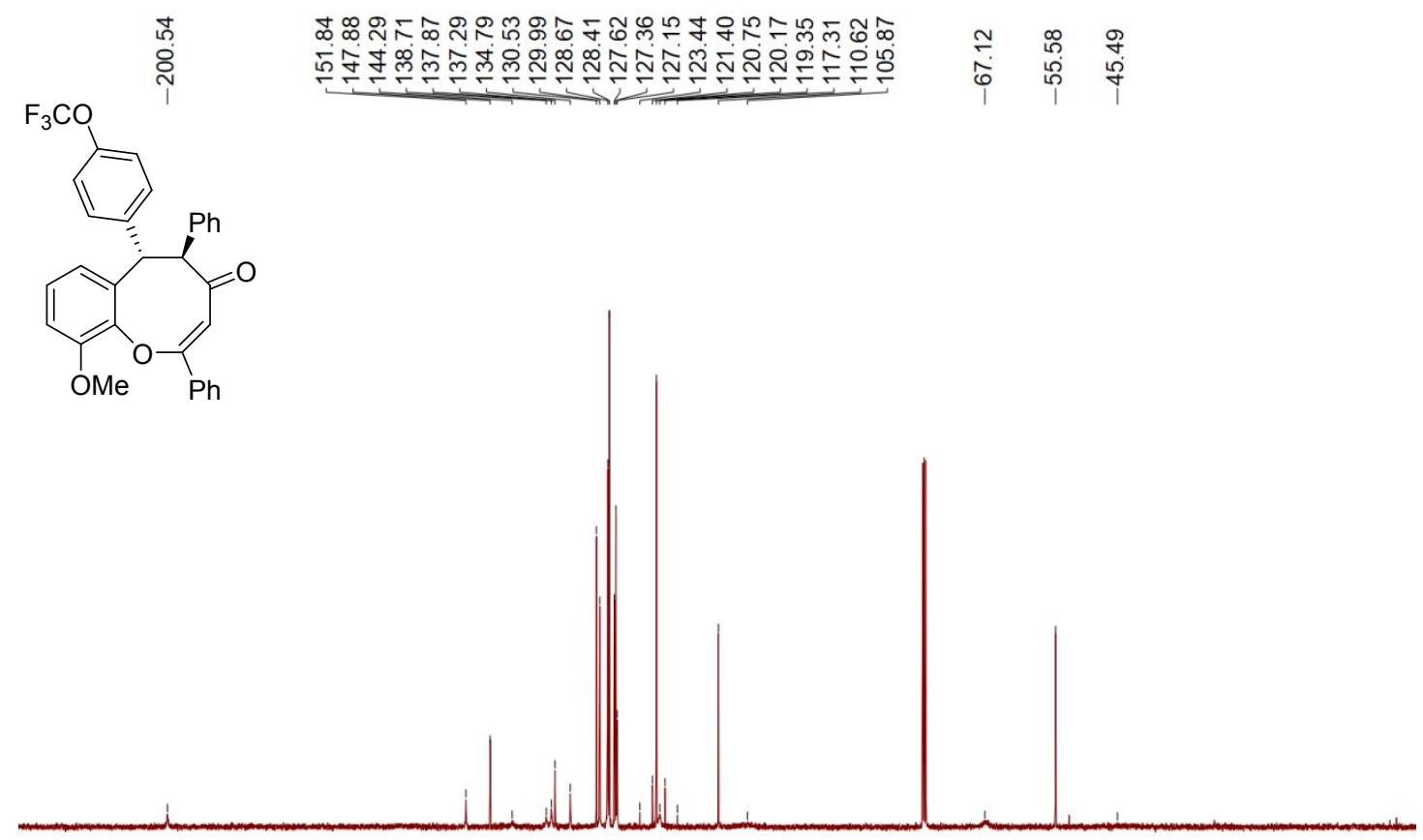

$\begin{array}{lllllllllllllllllllllll}220 & 210 & 200 & 190 & 180 & 170 & 160 & 150 & 140 & 130 & 120 & 110 & 100 & 90 & 80 & 70 & 60 & 50 & 40 & 30 & 20 & 10 & 0\end{array}$ 
${ }^{1} \mathrm{H}$ NMR of compound 4va (500 $\mathrm{MHz}$ in $\mathrm{CDCl}_{3}$ )

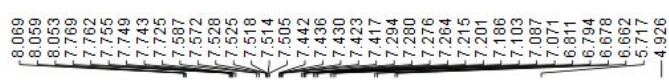<smiles>COc1cccc2c1O/C(c1ccccc1)=C\C(=O)C(c1ccccc1)[C@H]2c1ccc2ccccc2c1</smiles>

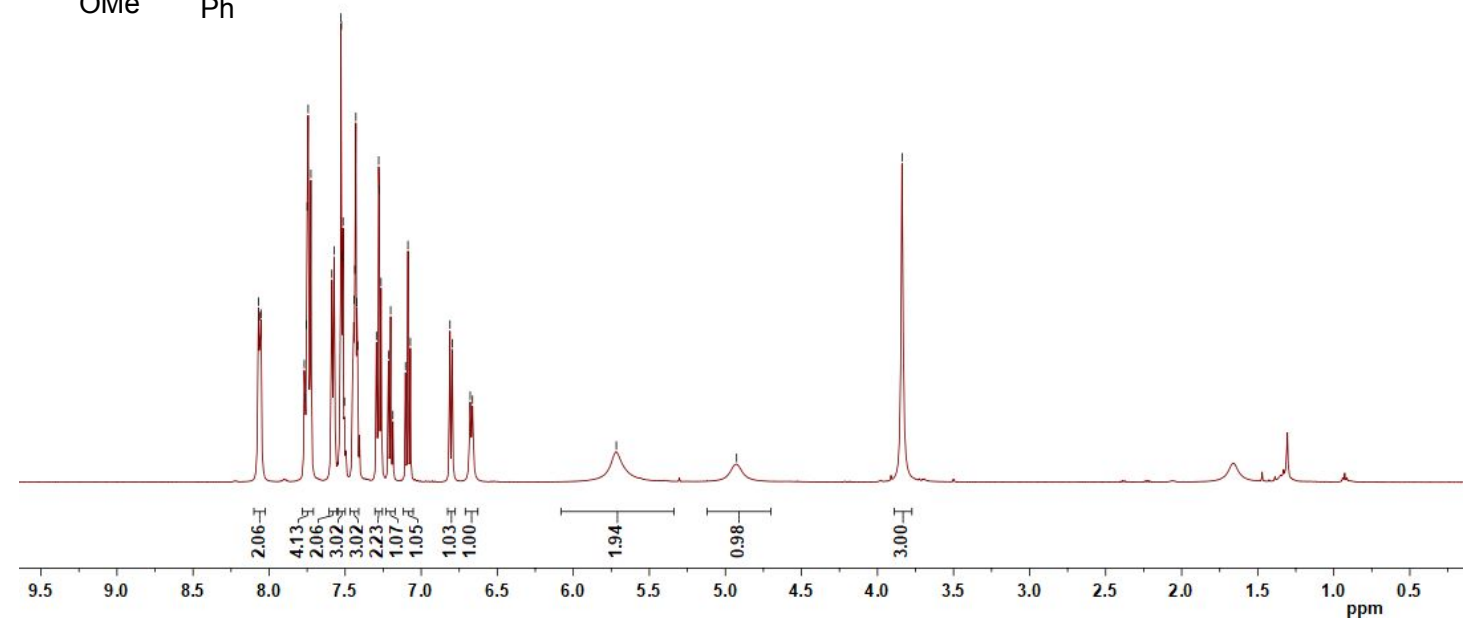

${ }^{13} \mathrm{C}$ NMR of compound 4va (126 $\mathrm{MHz}$ in $\mathrm{CDCl}_{3}$ )

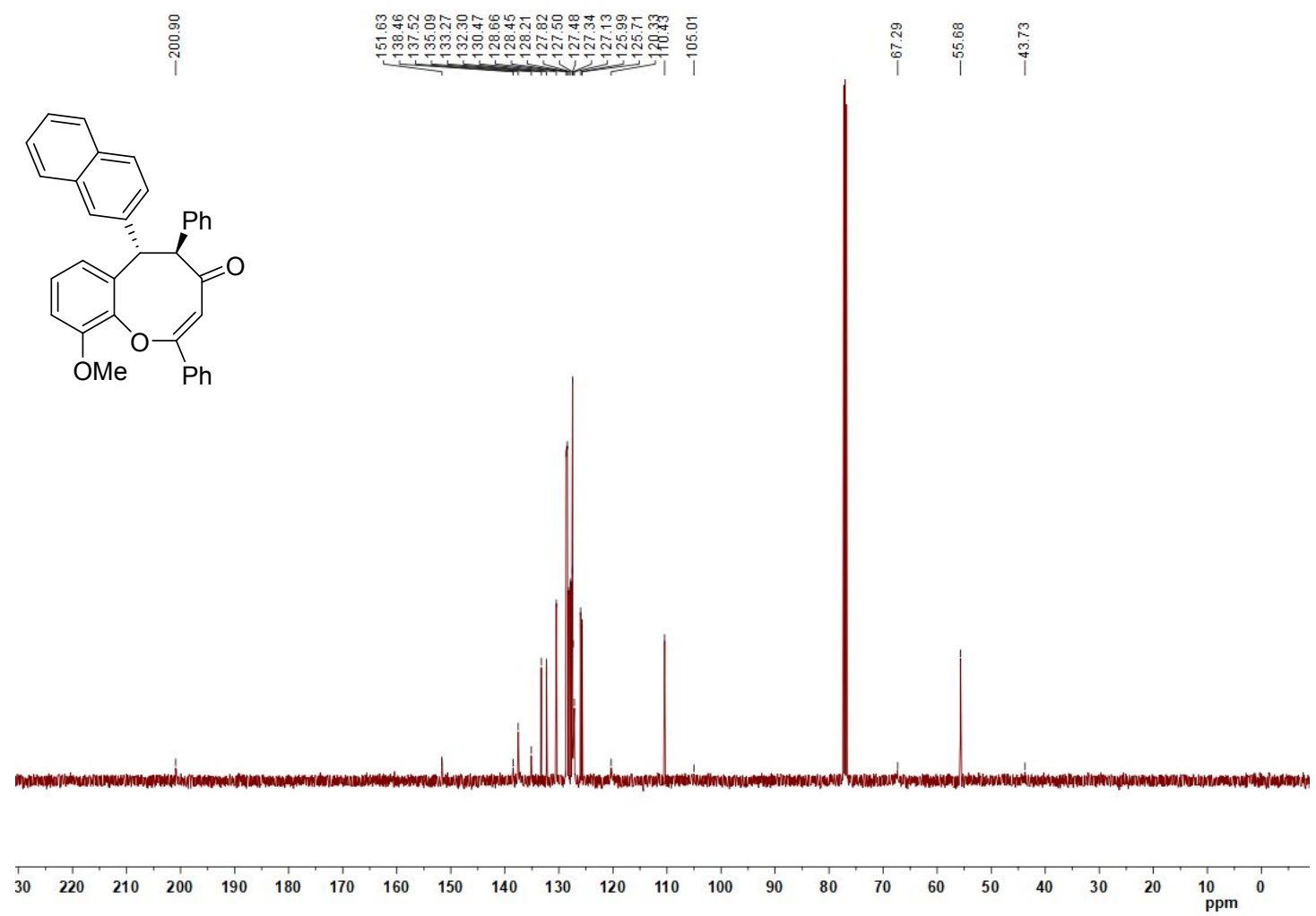


${ }^{1} \mathrm{H}$ NMR of compound 4wa (500 $\mathrm{MHz}$ in $\left.\mathrm{CDCl}_{3}\right)$

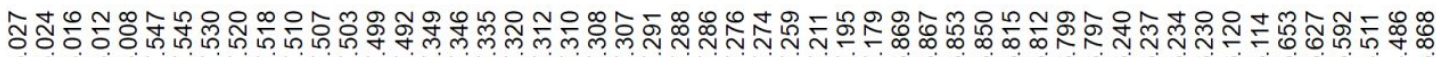

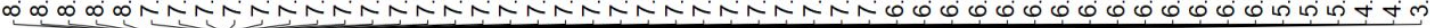<smiles>COc1cccc2c1O/C(c1ccccc1)=C\C(=O)[C@H](c1ccco1)[C@H]2c1ccccc1</smiles>

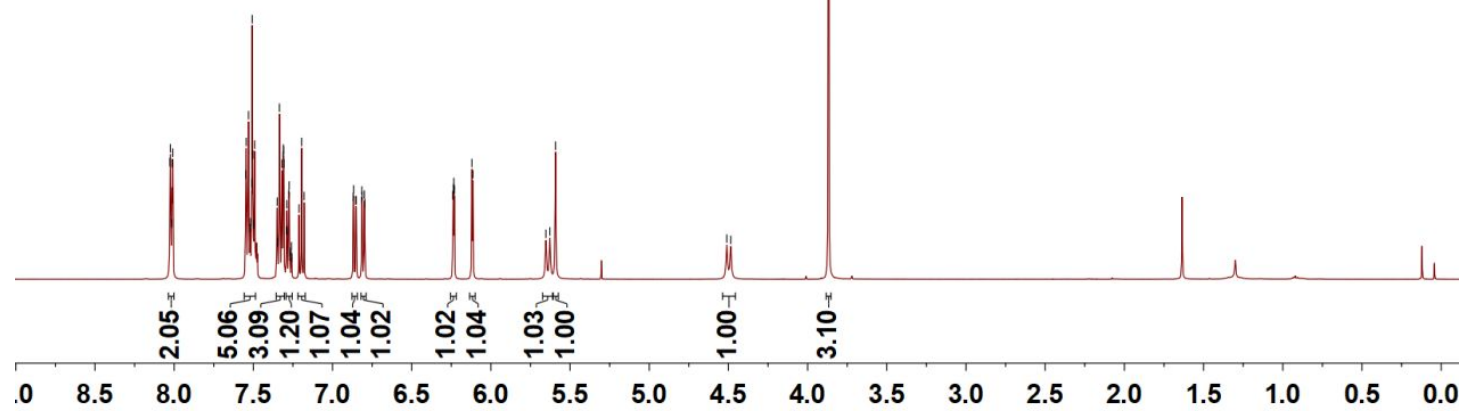

${ }^{13} \mathrm{C}$ NMR of compound 4wa (126 MHz in $\left.\mathrm{CDCl}_{3}\right)$<smiles>COc1cccc2c1O/C(c1ccccc1)=C\C(=O)C(c1ccccc1)[C@H]2c1ccco1</smiles>

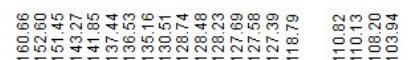

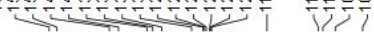

$\mathrm{OMe} \mathrm{Ph}$

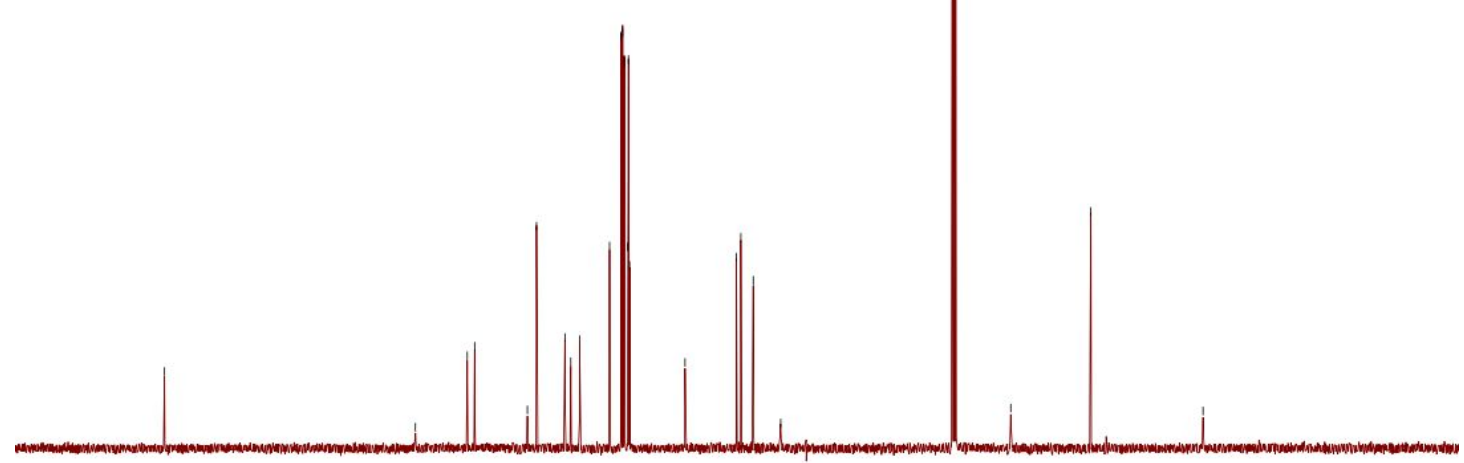

$\begin{array}{llllllllllllllllllllllllllll}1 & 120 & 210 & 200 & 190 & 180 & 170 & 160 & 150 & 140 & 130 & 120 & 110 & 100 & 90 & 80 & 70 & 60 & 50 & 40 & 30 & 20 & 1 & 1 & 1 \\ \end{array}$ 
${ }^{1} \mathrm{H}$ NMR of compound 4xa (600 $\mathrm{MHz}$ in $\left.\mathrm{CDCl}_{3}\right)$

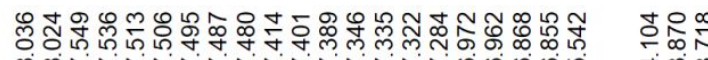

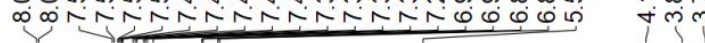

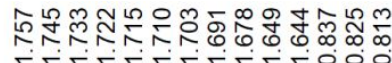<smiles>CC[C@H]1c2cccc(OC)c2O/C(c2ccccc2)=C\C(=O)C1c1ccccc1</smiles>

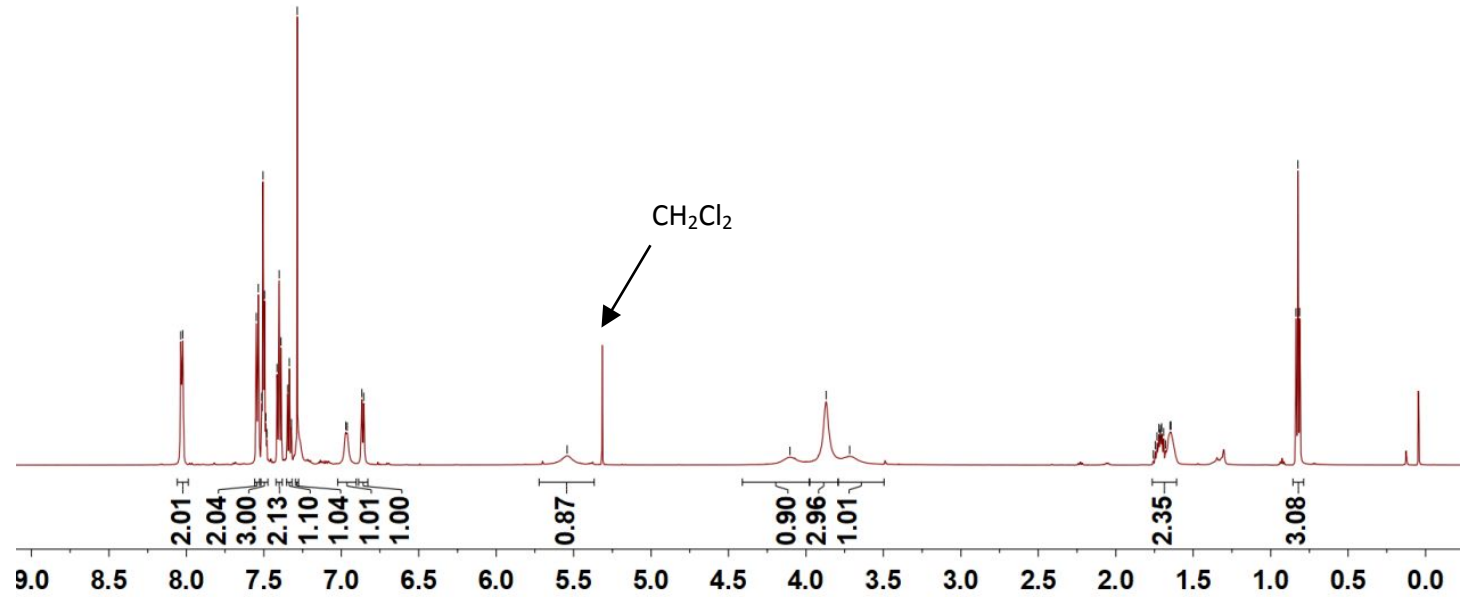

${ }^{13} \mathrm{C}$ NMR of compound 4xa (126 $\mathrm{MHz}$ in $\left.\mathrm{CDCl}_{3}\right)$<smiles>CCC1C(C)=CC(c2ccccc2)Oc2c(OC)cccc21</smiles>

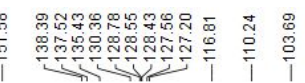


${ }^{1} \mathrm{H}$ NMR of compound $4 \mathrm{ab}\left(600 \mathrm{MHz}\right.$ in $\left.\mathrm{CDCl}_{3}\right)$

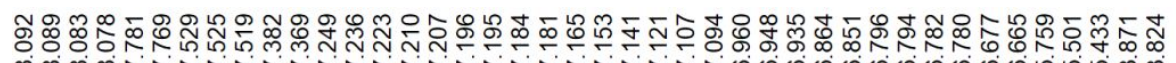<smiles>COc1ccccc1C1COC(=N)C(c2ccccc2)Oc2c(OC)cccc21</smiles>

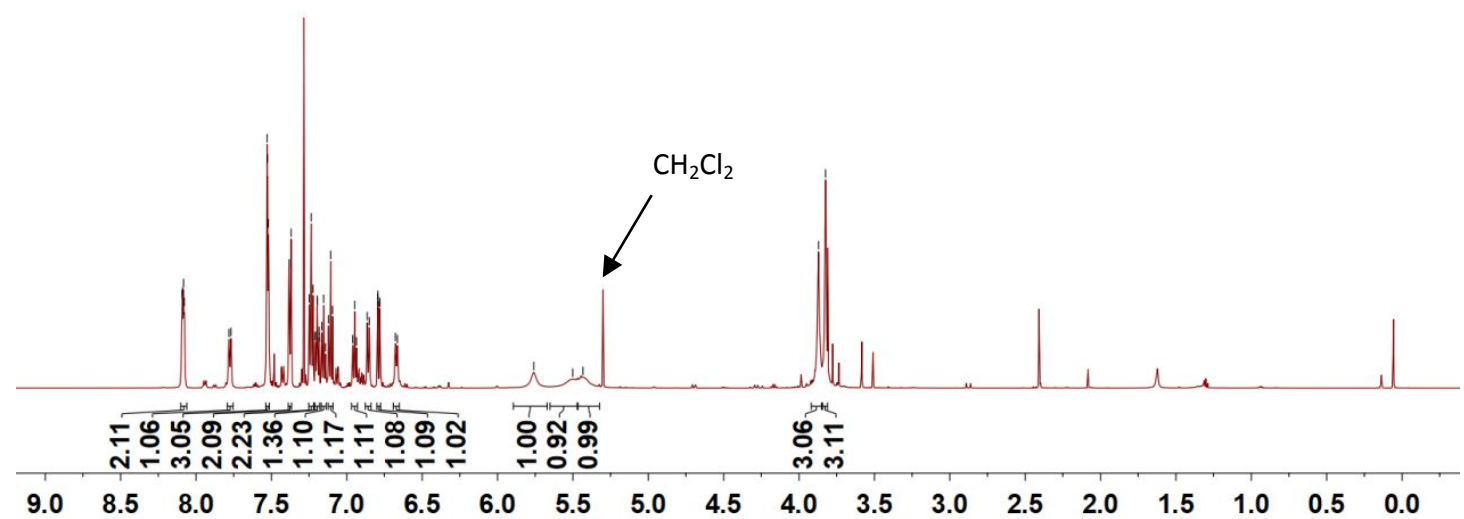

${ }^{13} \mathrm{C}$ NMR of compound 4ab (126 $\mathrm{MHz}$ in $\left.\mathrm{CDCl}_{3}\right)$

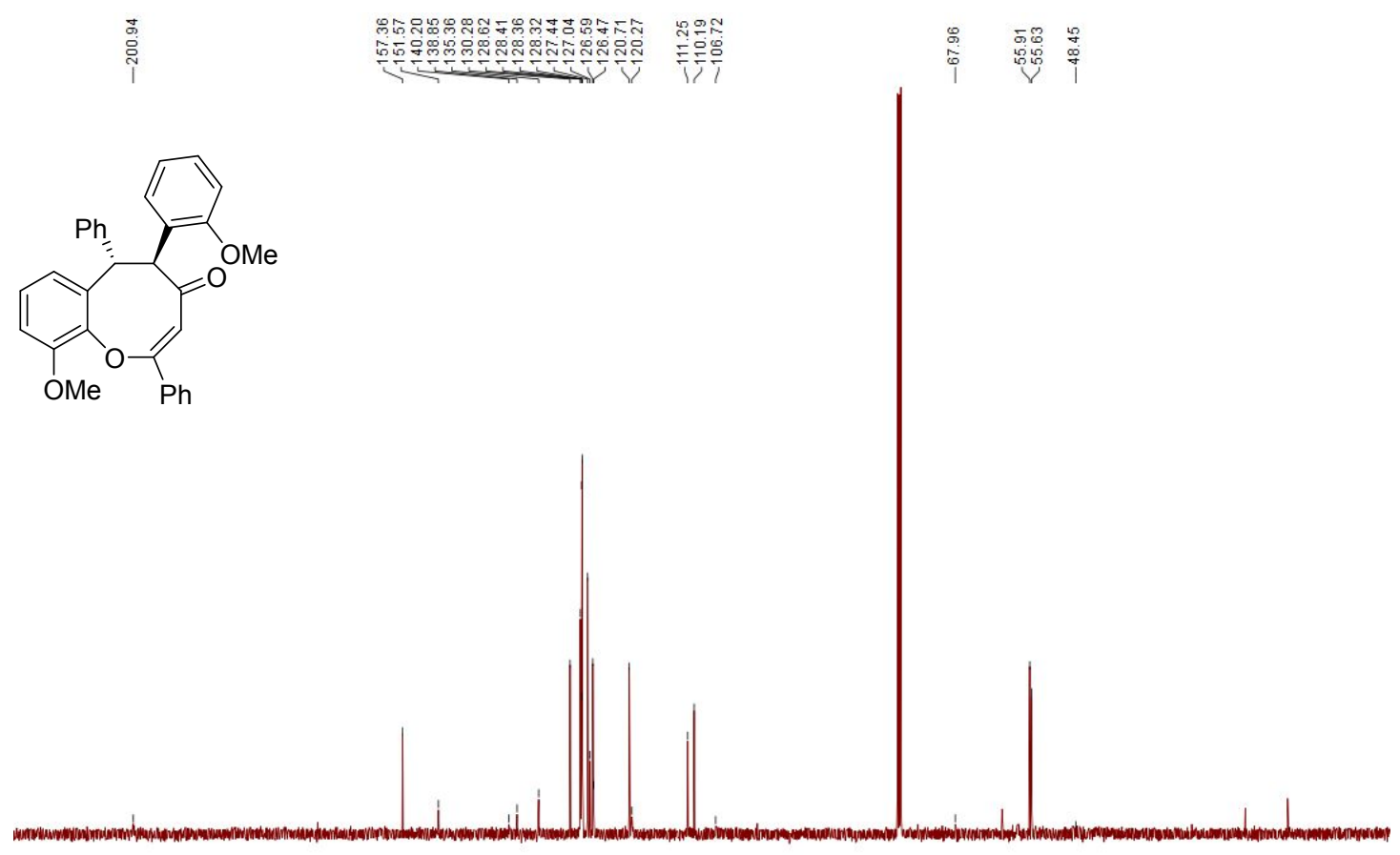

\begin{tabular}{llllllllllllllllllllllllllll}
\hline & 210 & 200 & 190 & 180 & 170 & 160 & 150 & 140 & 130 & 120 & 110 & 100 & 90 & 80 & 70 & 60 & 50 & 40 & 30 & 20 & 10 & 0
\end{tabular} 
${ }^{1} \mathrm{H}$ NMR of compound $4 \mathrm{ac}\left(500 \mathrm{MHz}\right.$ in $\left.\mathrm{CDCl}_{3}\right)$

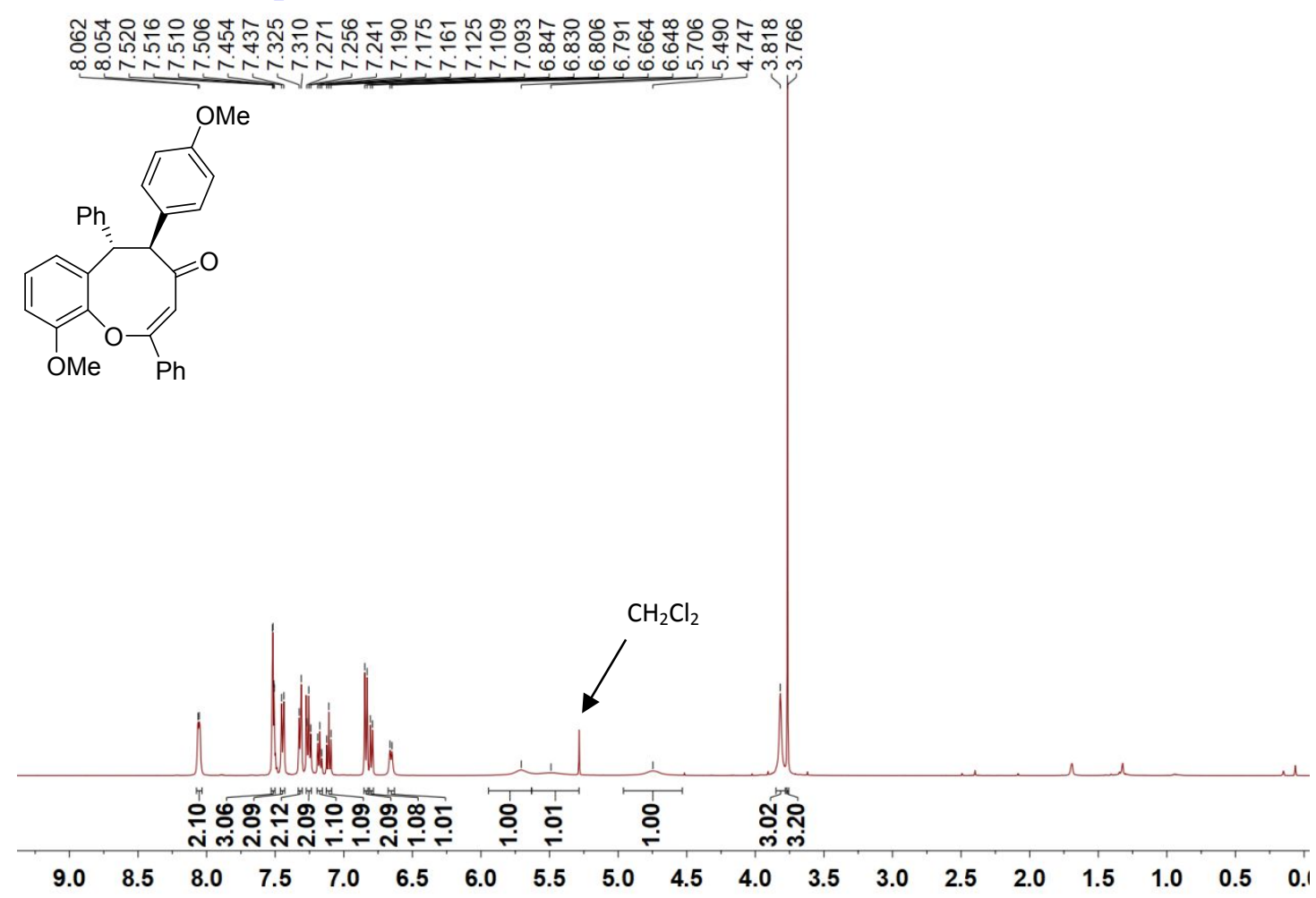

${ }^{13} \mathrm{C}$ NMR of compound $4 \mathrm{ac}\left(126 \mathrm{MHz}\right.$ in $\left.\mathrm{CDCl}_{3}\right)$

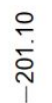

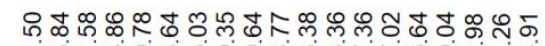

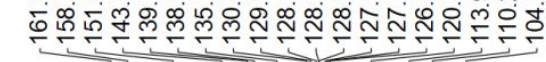<smiles>COc1ccc([C@H]2c3cccc(OC)c3O/C(c3ccccc3)=C\C(=O)[C@H]2c2ccccc2)cc1</smiles> 
${ }^{1} \mathrm{H}$ NMR of compound 4ad (500 $\mathrm{MHz}$ in $\left.\mathrm{CDCl}_{3}\right)$

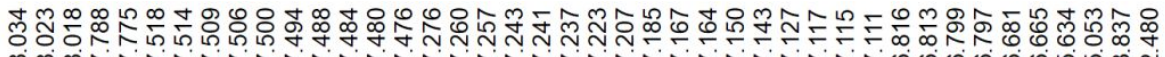

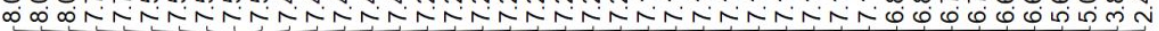<smiles>COc1cccc2c1OC(c1ccccc1)C=CC2=O</smiles>

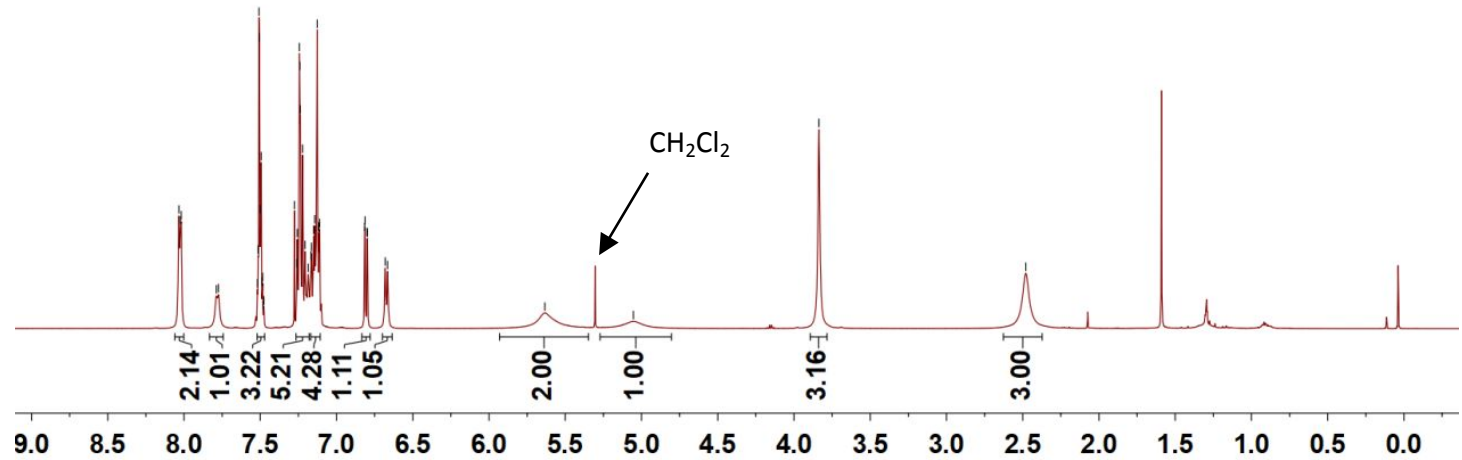

${ }^{13} \mathrm{C}$ NMR of compound $4 \mathrm{ad}\left(126 \mathrm{MHz}\right.$ in $\left.\mathrm{CDCl}_{3}\right)$<smiles>COc1cccc2c1OC(c1ccccc1)CC(=O)C(c1ccccc1)C2c1ccccc1</smiles> 
${ }^{1} \mathrm{H}$ NMR of compound 4ae (500 $\mathrm{MHz}$ in $\left.\mathrm{CDCl}_{3}\right)$

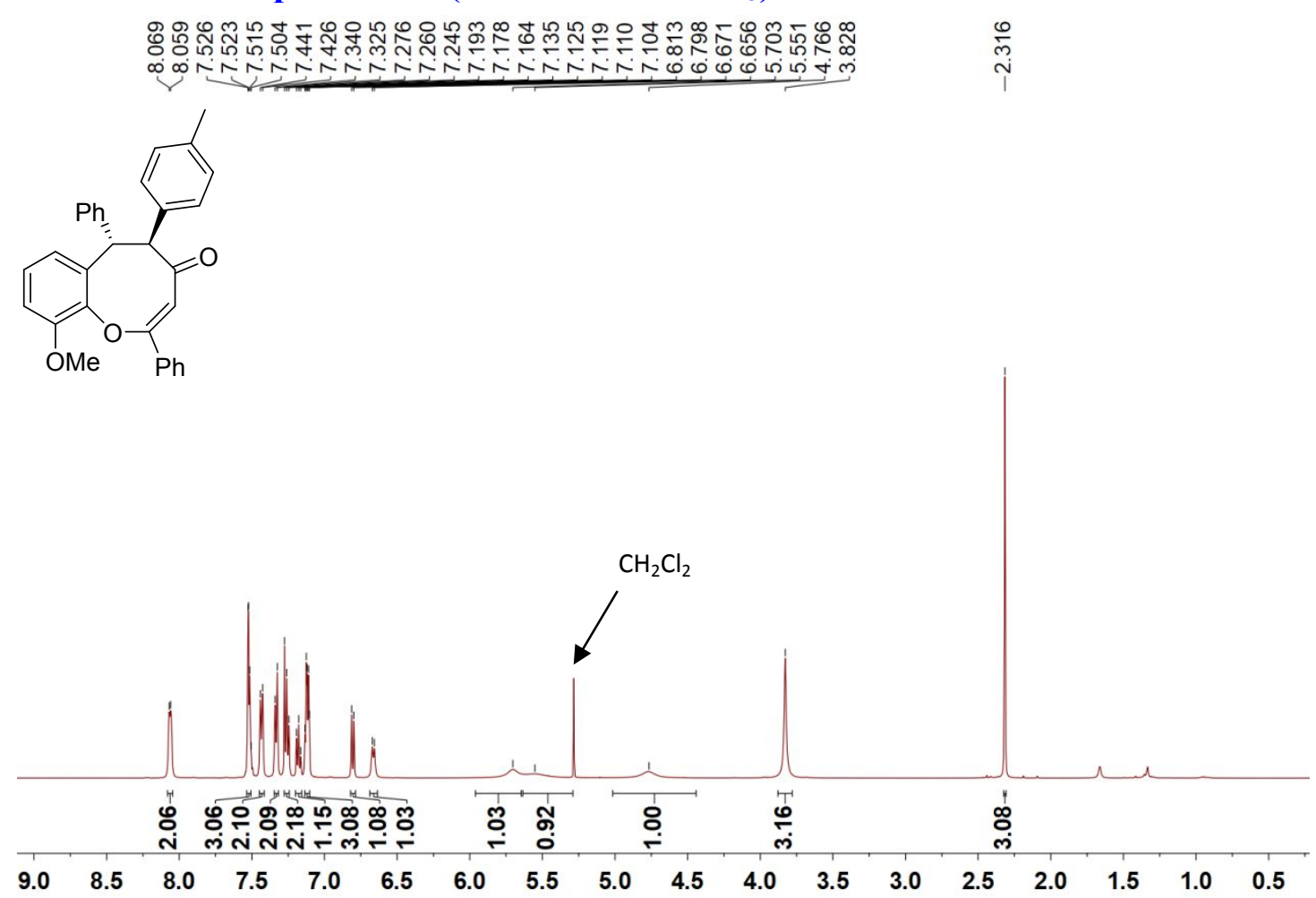

${ }^{13} \mathrm{C}$ NMR of compound 4ae (126 $\mathrm{MHz}$ in $\left.\mathrm{CDCl}_{3}\right)$

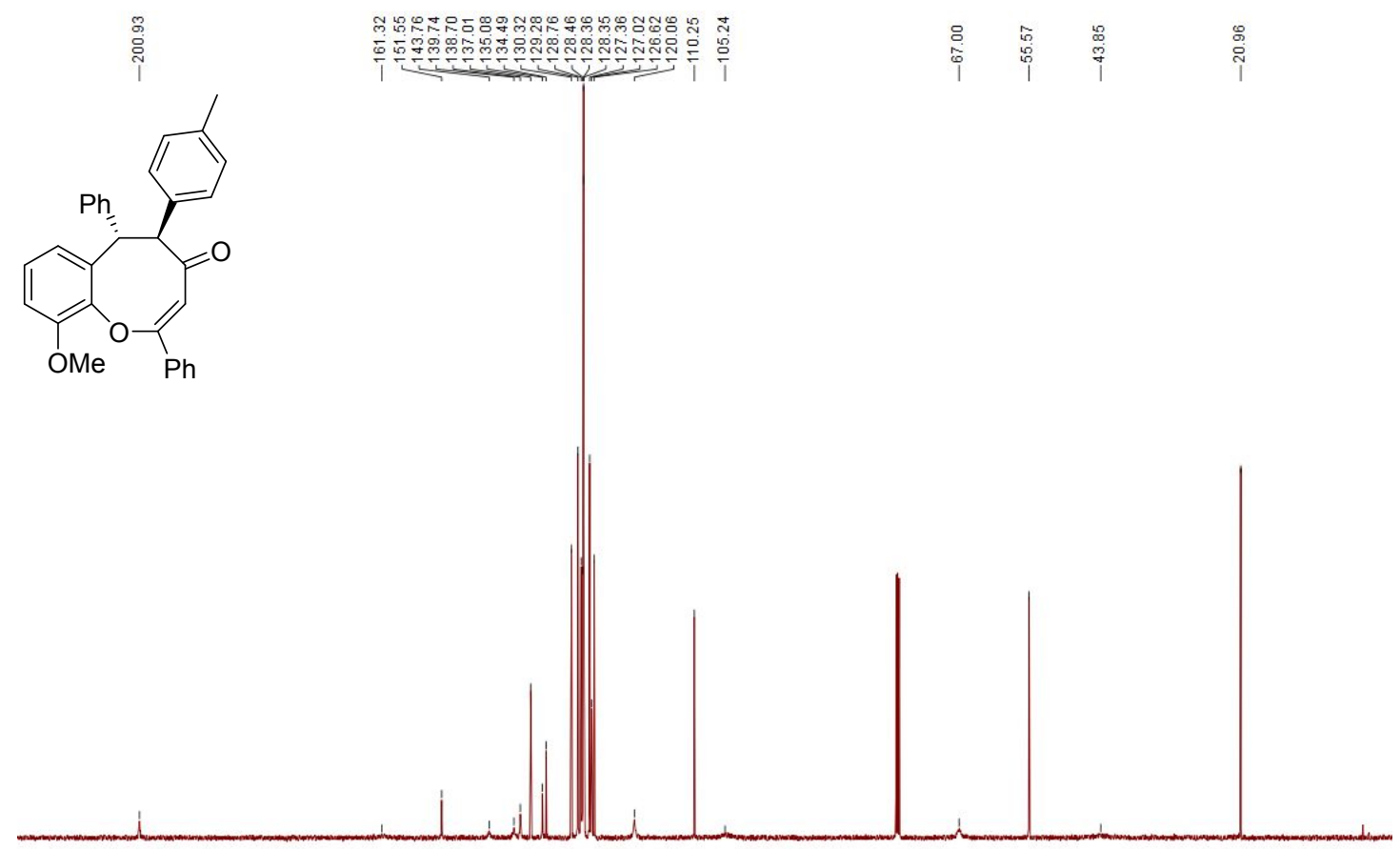

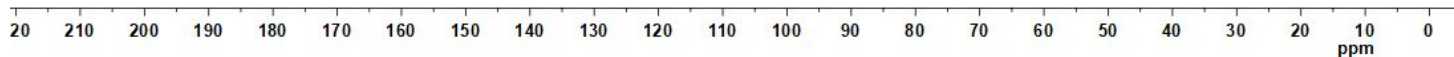


${ }^{1} \mathrm{H}$ NMR of compound 4 af (500 $\mathrm{MHz}$ in $\mathrm{CDCl}_{3}$ )

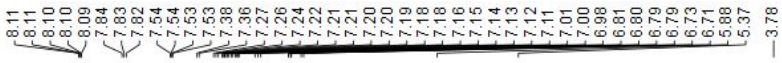<smiles>COc1cccc2c1OC(c1ccccc1)CC(=O)C2c1ccccc1F</smiles>

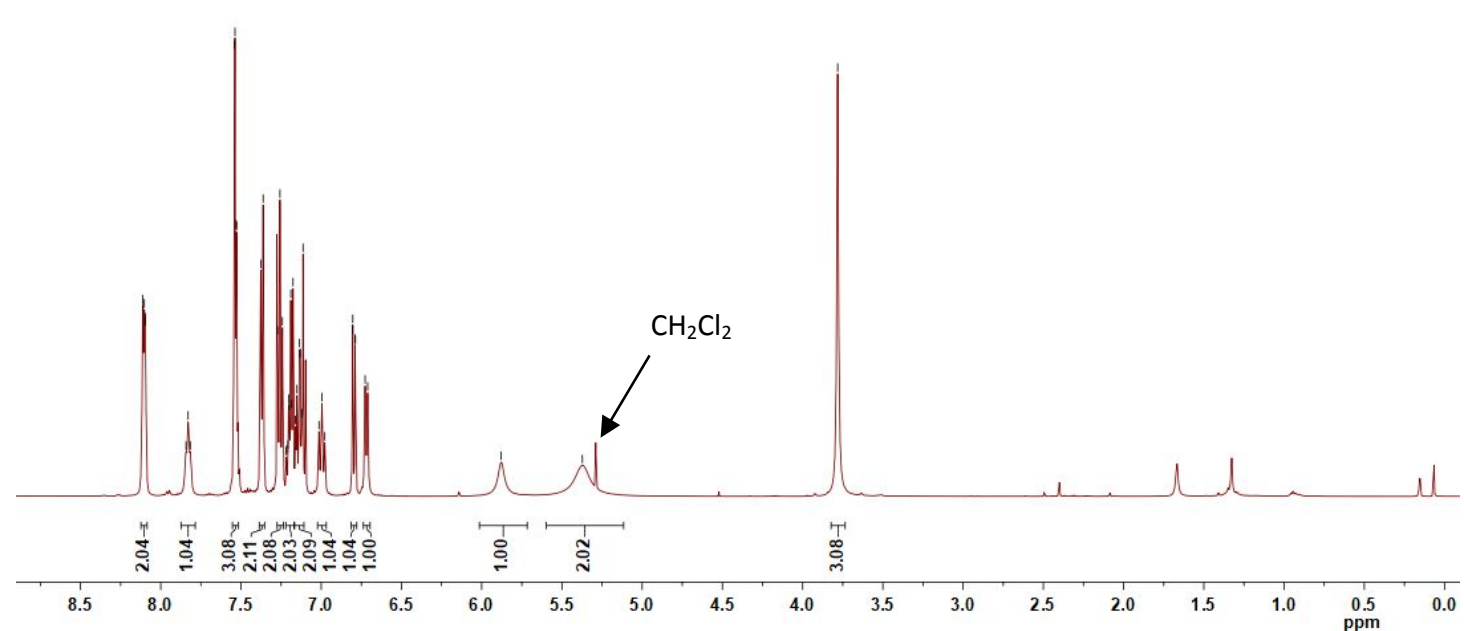

${ }^{13} \mathrm{C}$ NMR of compound 4 af $\left(126 \mathrm{MHz}\right.$ in $\left.\mathrm{CDCl}_{3}\right)$

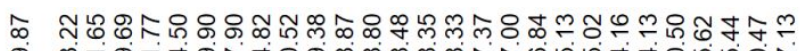

要

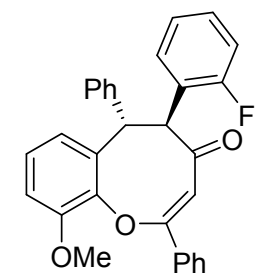

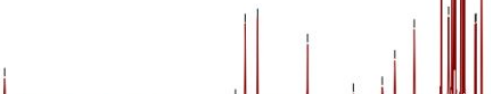


${ }^{1} \mathrm{H}$ NMR of compound $4 \mathrm{ag}\left(500 \mathrm{MHz}\right.$ in $\left.\mathrm{CDCl}_{3}\right)$

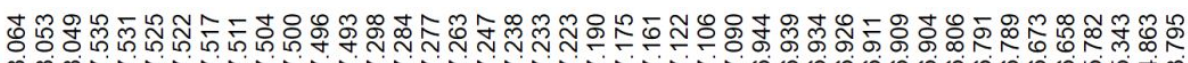

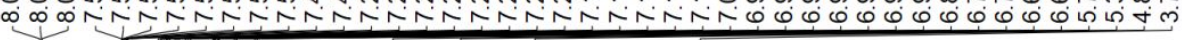<smiles>COc1cccc2c1OC(c1ccccc1)C=C(c1ccccc1)C(=O)/C=C\2c1cccc(F)c1</smiles>

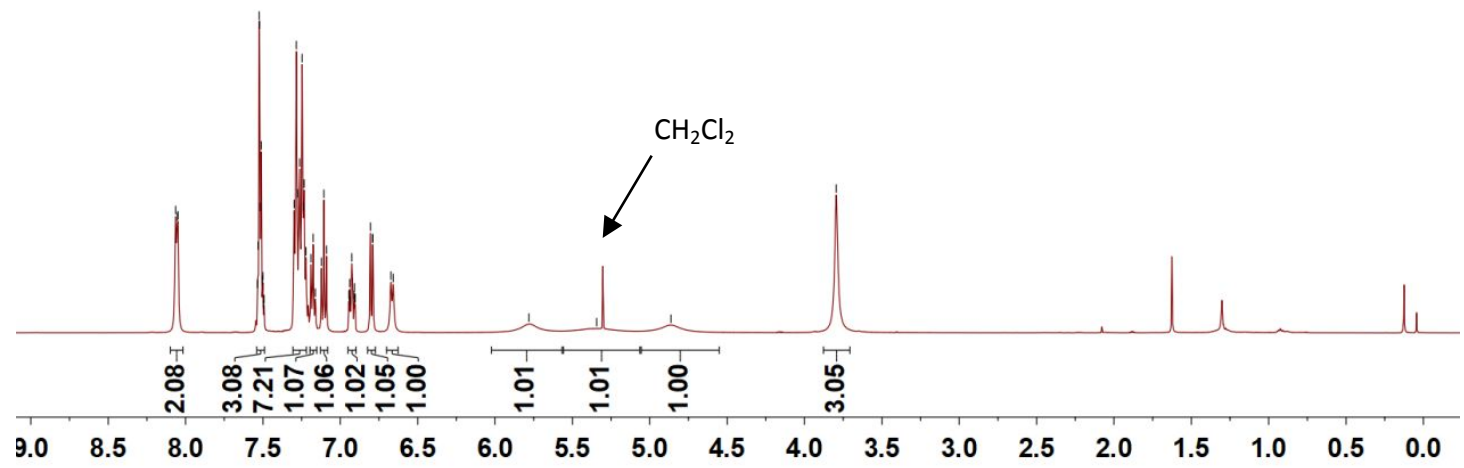

${ }^{13} \mathrm{C}$ NMR of compound $4 \mathrm{ag}\left(126 \mathrm{MHz}\right.$ in $\left.\mathrm{CDCl}_{3}\right)$

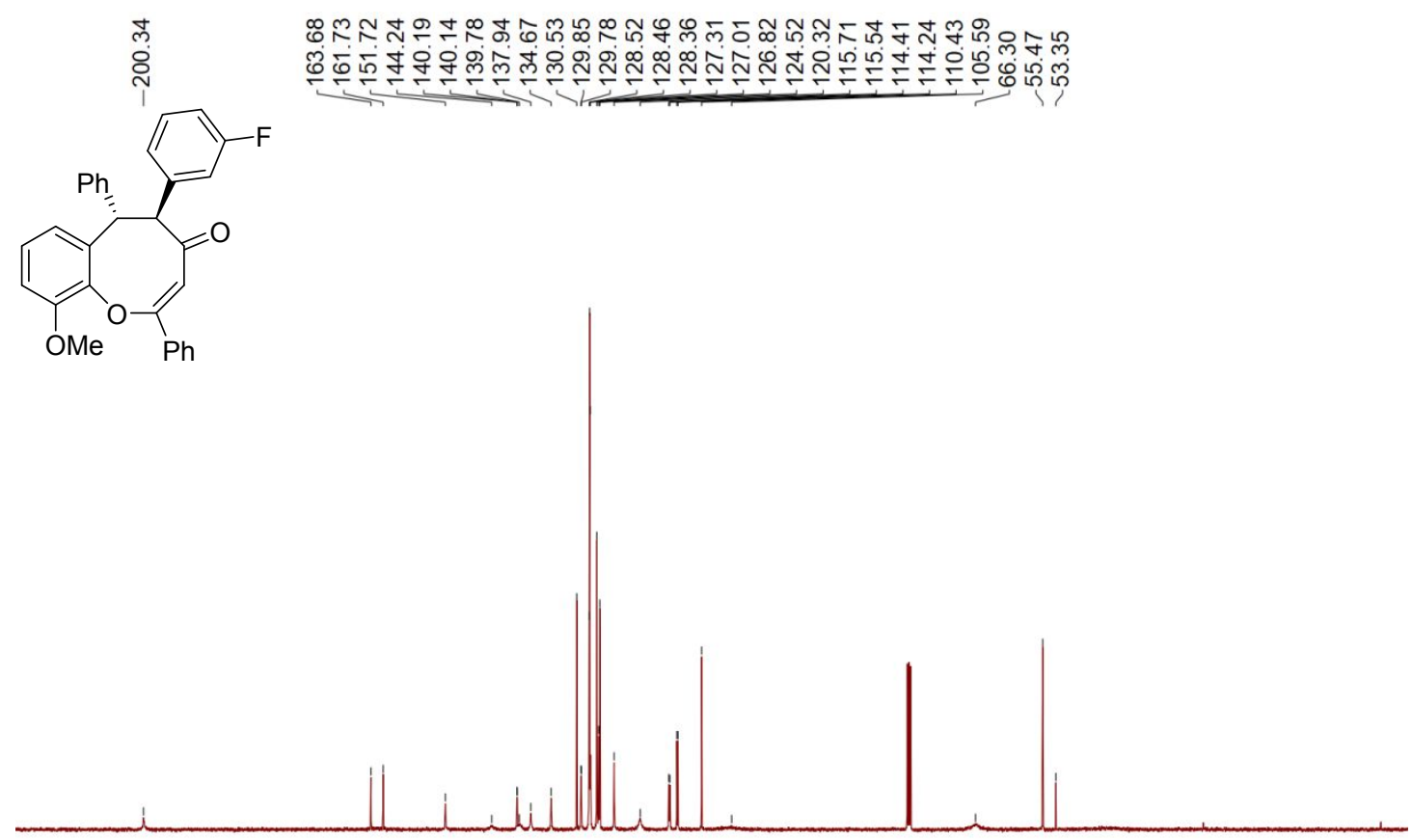

$\begin{array}{lllllllllllllllllllllll}20 & 210 & 200 & 190 & 180 & 170 & 160 & 150 & 140 & 130 & 120 & 110 & 100 & 90 & 80 & 70 & 60 & 50 & 40 & 30 & 20 & 10 & 0\end{array}$ 
${ }^{1} \mathrm{H}$ NMR of compound 4ah (500 $\mathrm{MHz}$ in $\left.\mathrm{CDCl}_{3}\right)$
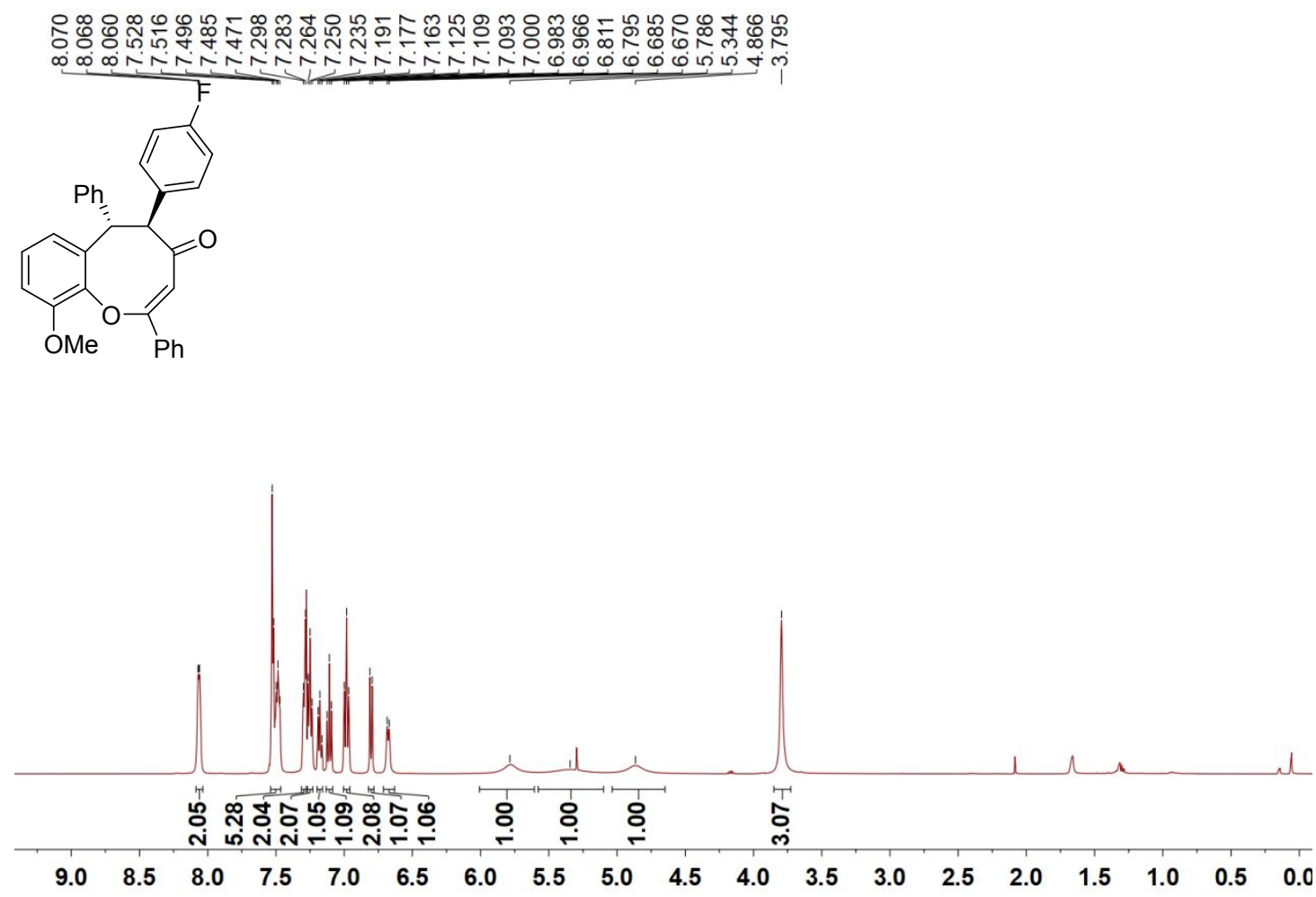

${ }^{13} \mathrm{C}$ NMR of compound 4ah (126 $\mathrm{MHz}$ in $\left.\mathrm{CDCl}_{3}\right)$

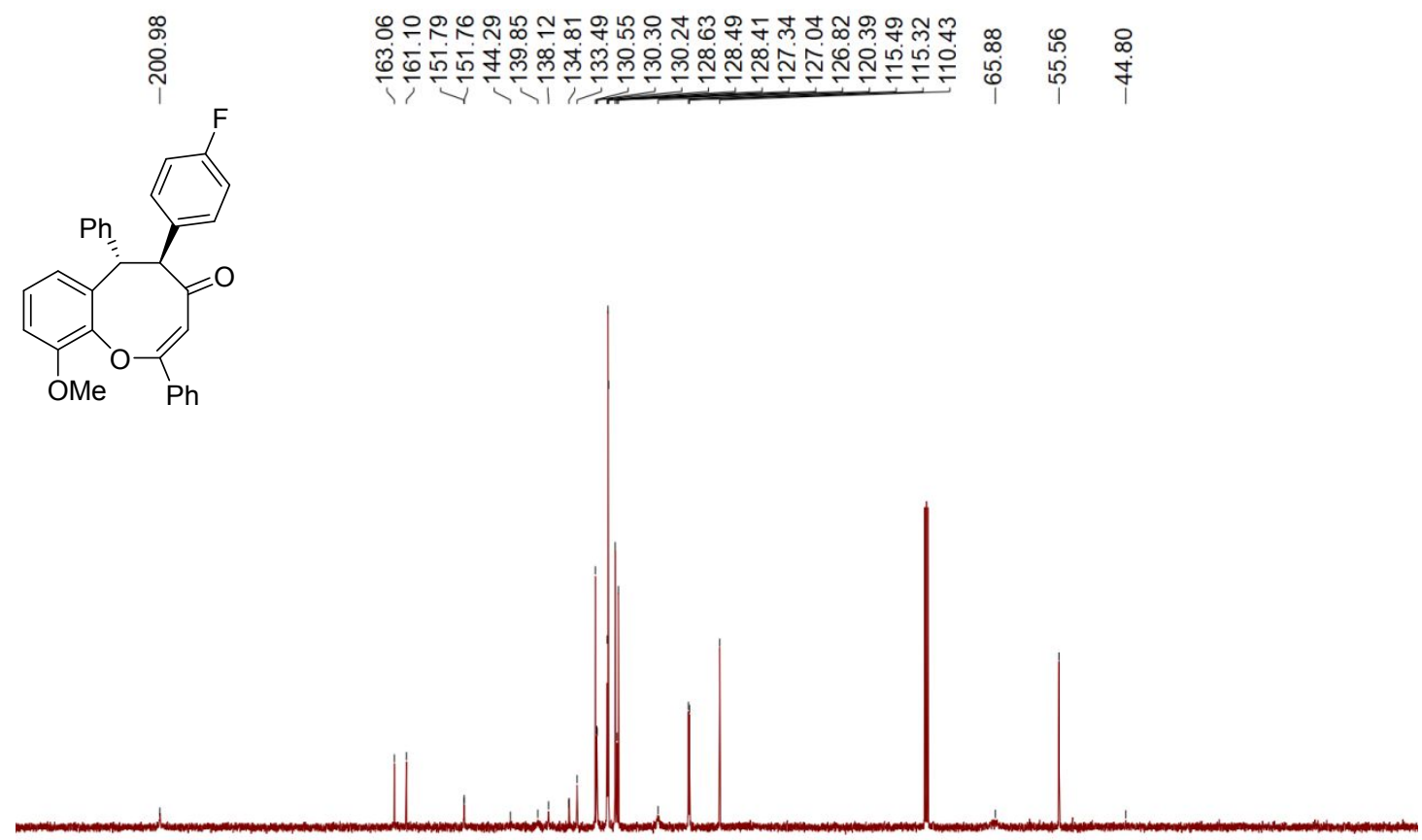

$\begin{array}{lllllllllllllllllllllll}220 & 210 & 200 & 190 & 180 & 170 & 160 & 150 & 140 & 130 & 120 & 110 & 100 & 90 & 80 & 70 & 60 & 50 & 40 & 30 & 20 & 10 & 0\end{array}$ 
${ }^{1} \mathrm{H}$ NMR of compound 4ai (500 $\mathrm{MHz}$ in $\left.\mathrm{CDCl}_{3}\right)$

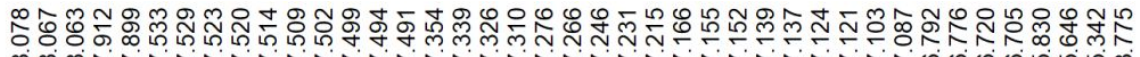

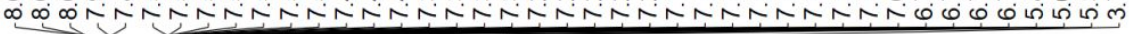<smiles>COc1cccc2c1OC(c1ccccc1)CC(=O)C2c1ccccc1Cl</smiles>

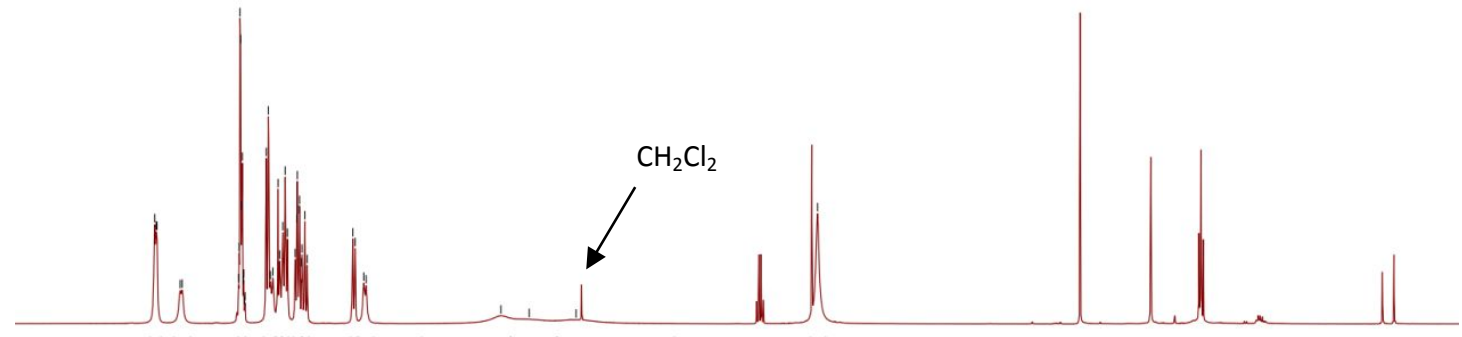

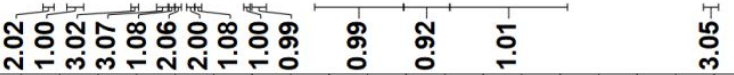

$\begin{array}{llllllllllllllllll}8.5 & 8.0 & 7.5 & 7.0 & 6.5 & 6.0 & 5.5 & 5.0 & 4.5 & 4.0 & 3.5 & 3.0 & 2.5 & 2.0 & 1.5 & 1.0 & 0.5 & 0.0\end{array}$

${ }^{13} \mathrm{C}$ NMR of compound 4ai (126 $\mathrm{MHz}$ in $\mathrm{CDCl}_{3}$ )

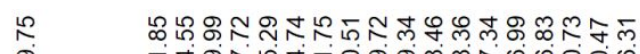

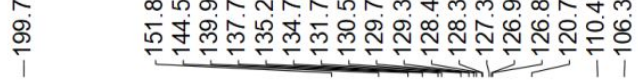

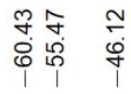<smiles>COc1cccc2c1OC(c1ccccc1)CC(=O)C2c1ccccc1Cl</smiles>

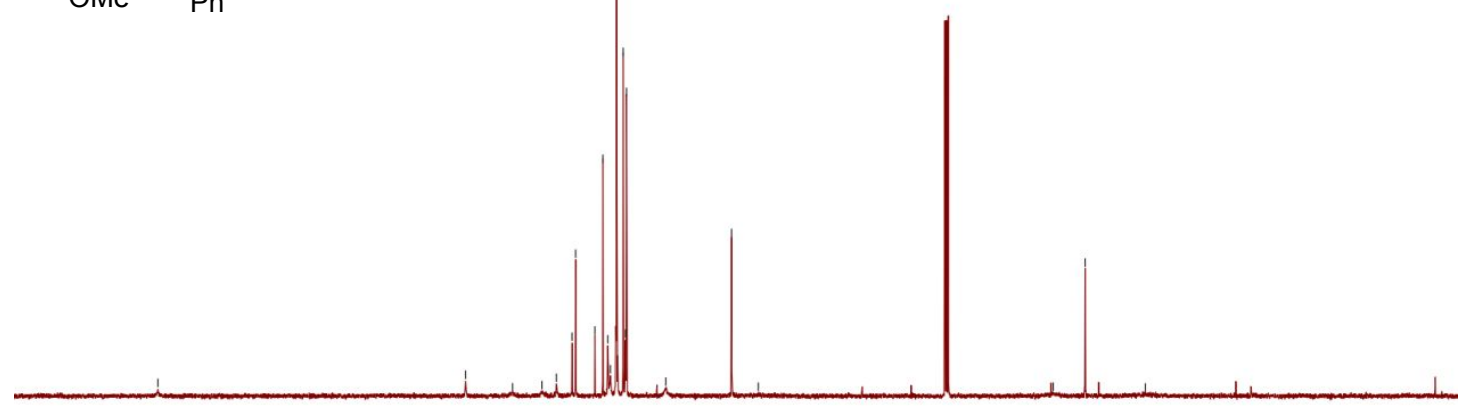

$\begin{array}{lllllllllllllllllllllll}20 & 210 & 200 & 190 & 180 & 170 & 160 & 150 & 140 & 130 & 120 & 110 & 100 & 90 & 80 & 70 & 60 & 50 & 40 & 30 & 20 & 10 & 0\end{array}$ 
${ }^{1} \mathrm{H}$ NMR of compound 4aj (500 $\mathrm{MHz}$ in $\left.\mathrm{CDCl}_{3}\right)$

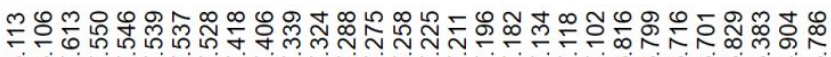

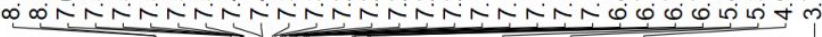<smiles>COc1cccc2c1OC(c1ccccc1)C=CC2=O</smiles>

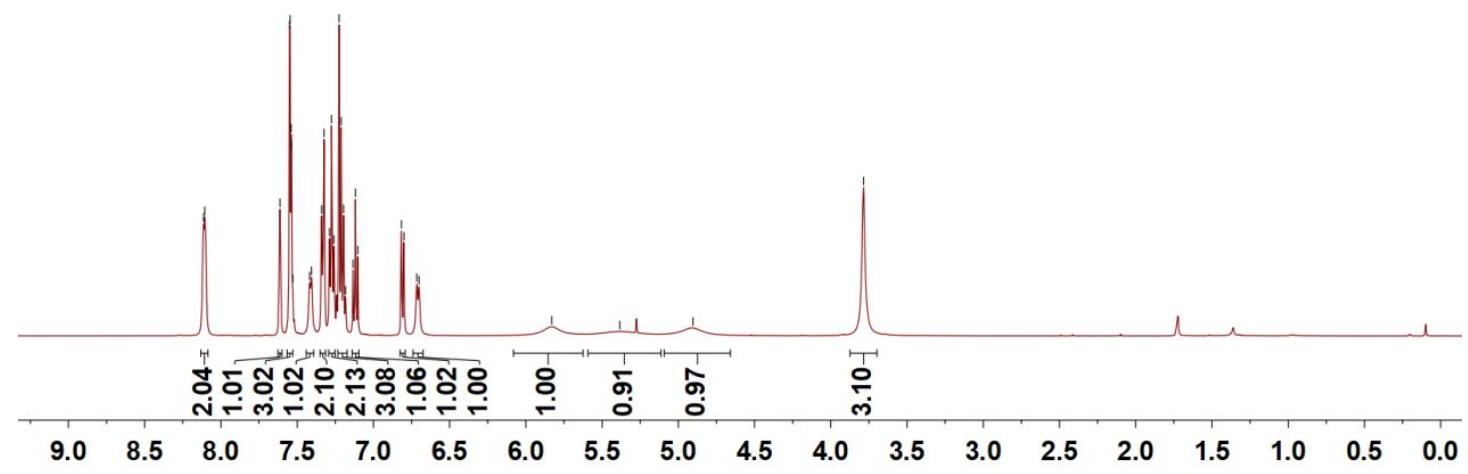

${ }^{13} \mathrm{C}$ NMR of compound 4aj (126 $\mathrm{MHz}$ in $\mathrm{CDCl}_{3}$ )

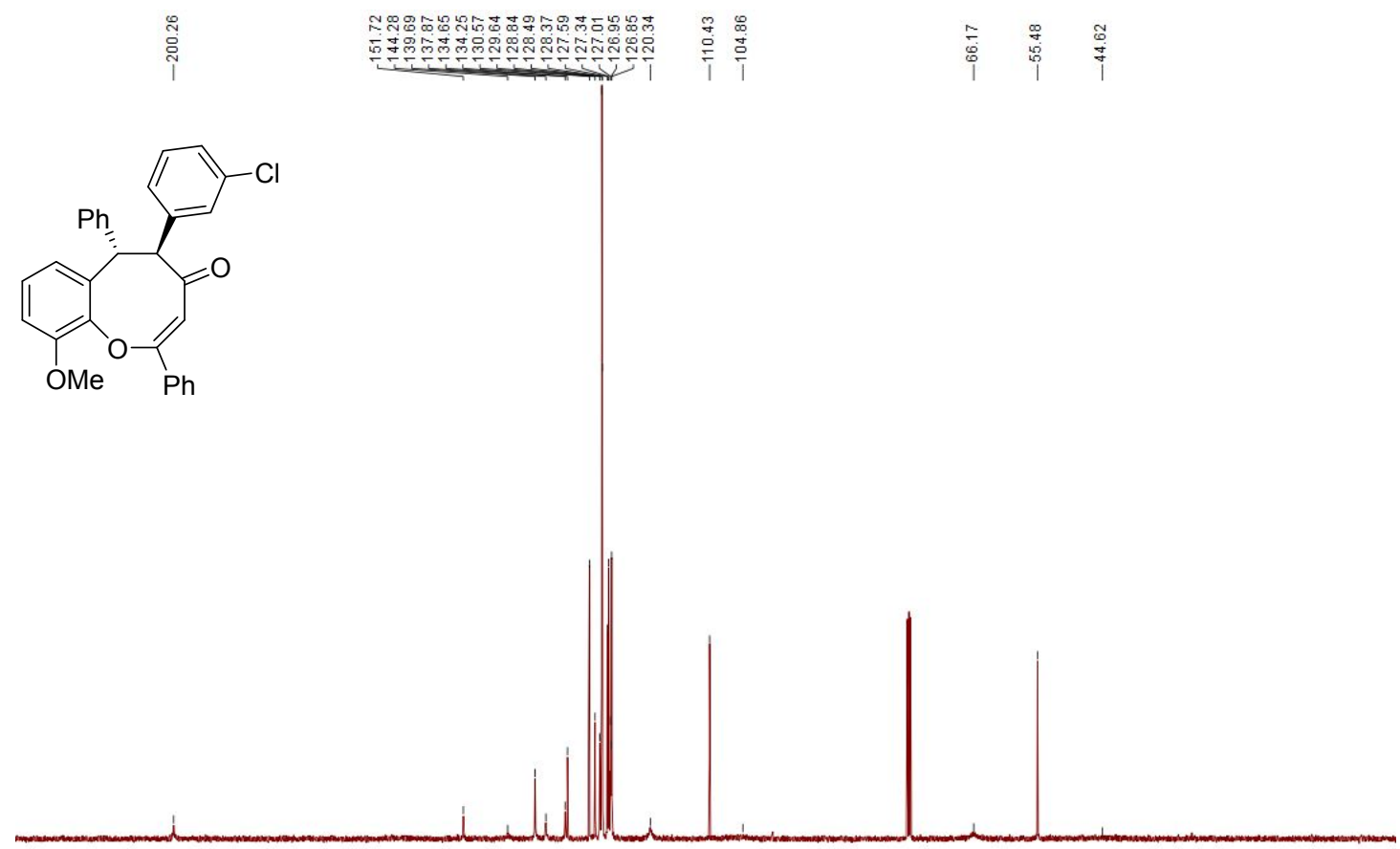

$\begin{array}{llllllllllllllllllllllllllll}220 & 210 & 200 & 190 & 180 & 170 & 160 & 150 & 140 & 130 & 120 & 110 & 100 & 90 & 80 & 70 & 60 & 50 & 40 & 30 & 20 & 10 & 0 \\ \mathrm{ppm} & \end{array}$ 
${ }^{1} \mathrm{H}$ NMR of compound 4ak (500 $\mathrm{MHz}$ in $\left.\mathrm{CDCl}_{3}\right)$

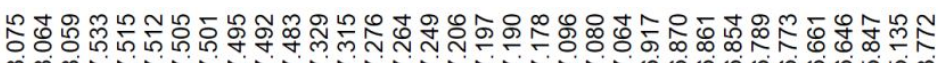

on<smiles>COc1cccc2c1OC(c1ccccc1)C=C(c1cccs1)C(c1ccccc1)C2=O</smiles>

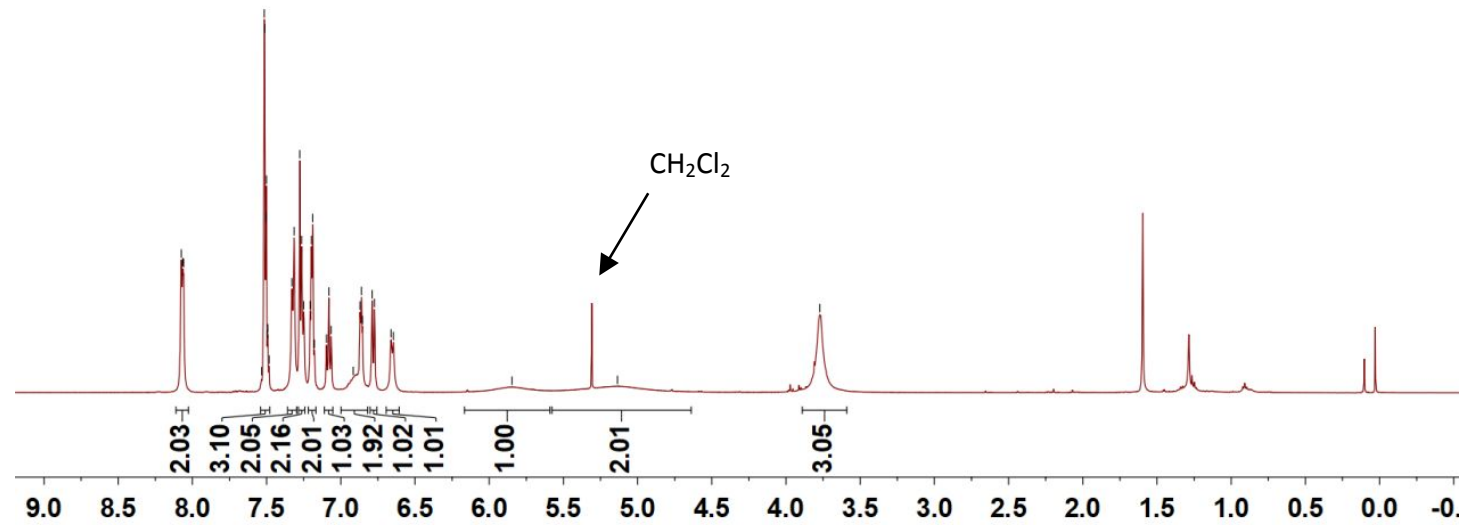

${ }^{13} \mathrm{C}$ NMR of compound 4ak (126 $\mathrm{MHz}$ in $\left.\mathrm{CDCl}_{3}\right)$

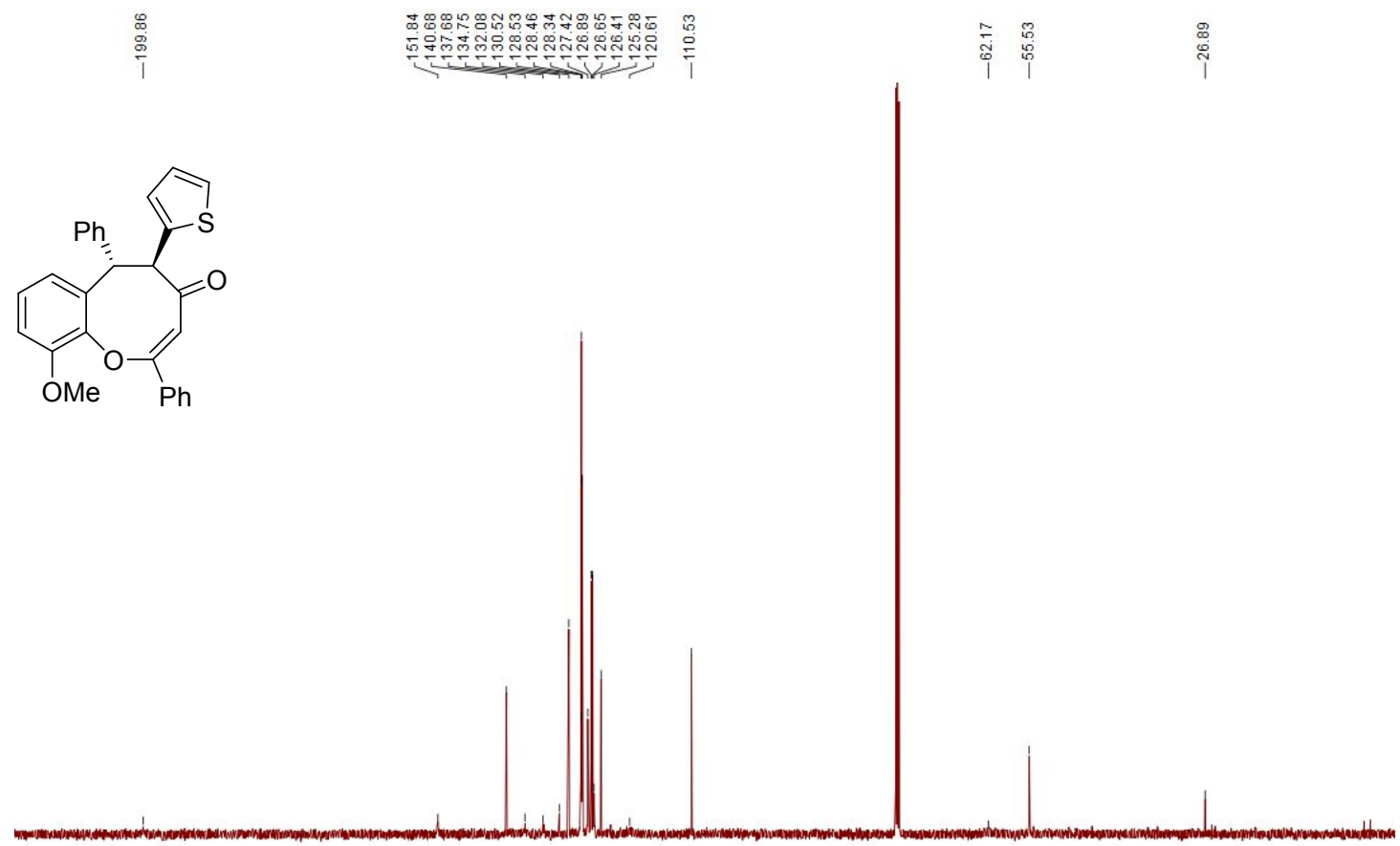

\begin{tabular}{llllllllllllllllllllllll}
\hline 20 & 210 & 200 & 190 & 180 & 170 & 160 & 150 & 140 & 130 & 120 & 110 & 100 & 90 & 80 & 70 & 60 & 50 & 40 & 30 & 20 & 10 & 0 \\
$\mathrm{ppm}$ & &
\end{tabular} 
${ }^{1} \mathrm{H}$ NMR of compound 4al (600 $\mathrm{MHz}$ in $\left.\mathrm{CDCl}_{3}\right)$

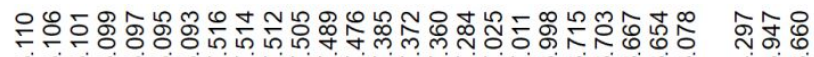

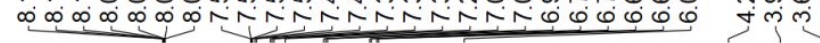<smiles>COc1cccc2c1O/C(c1ccccc1)=C\C(=O)C(C)C2c1ccccc1</smiles>

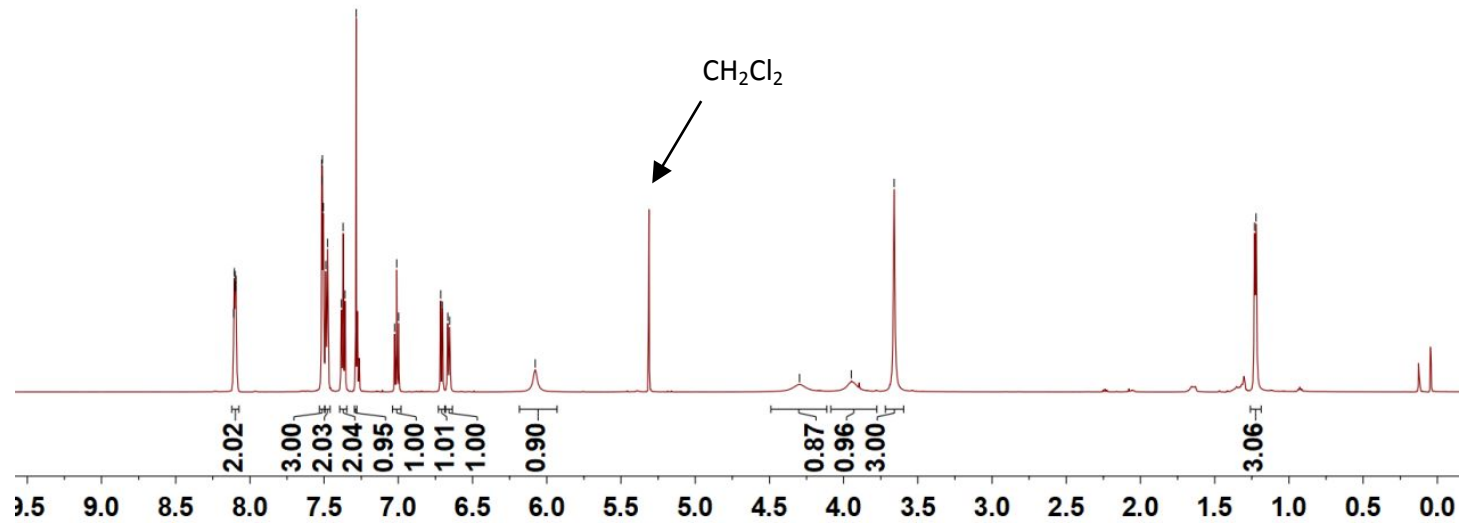

${ }^{13} \mathrm{C}$ NMR of compound 4al (151 $\mathrm{MHz}$ in $\mathrm{CDCl}_{3}$ )
离

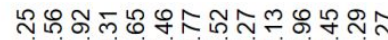

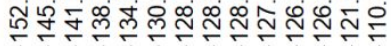
乐 乎电
นก๊
$\stackrel{\text { 울 }}{\stackrel{1}{1}}$<smiles>COc1cccc2c1O/C(c1ccccc1)=C\C(=O)C(C)C2c1ccccc1</smiles>

$\begin{array}{llllllllllllllllllllll}210 & 200 & 190 & 180 & 170 & 160 & 150 & 140 & 130 & 120 & 110 & 100 & 90 & 80 & 70 & 60 & 50 & 40 & 30 & 20 & 10 & 0\end{array}$ 
${ }^{1} \mathrm{H}$ NMR of compound $4 \mathrm{am}\left(500 \mathrm{MHz}\right.$ in $\left.\mathrm{CDCl}_{3}\right)$

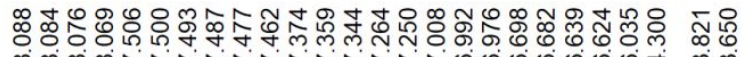

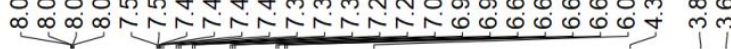<smiles>CCCC1C(=O)C=C(c2ccccc2)Oc2c(OC)cccc2C1c1ccccc1</smiles>

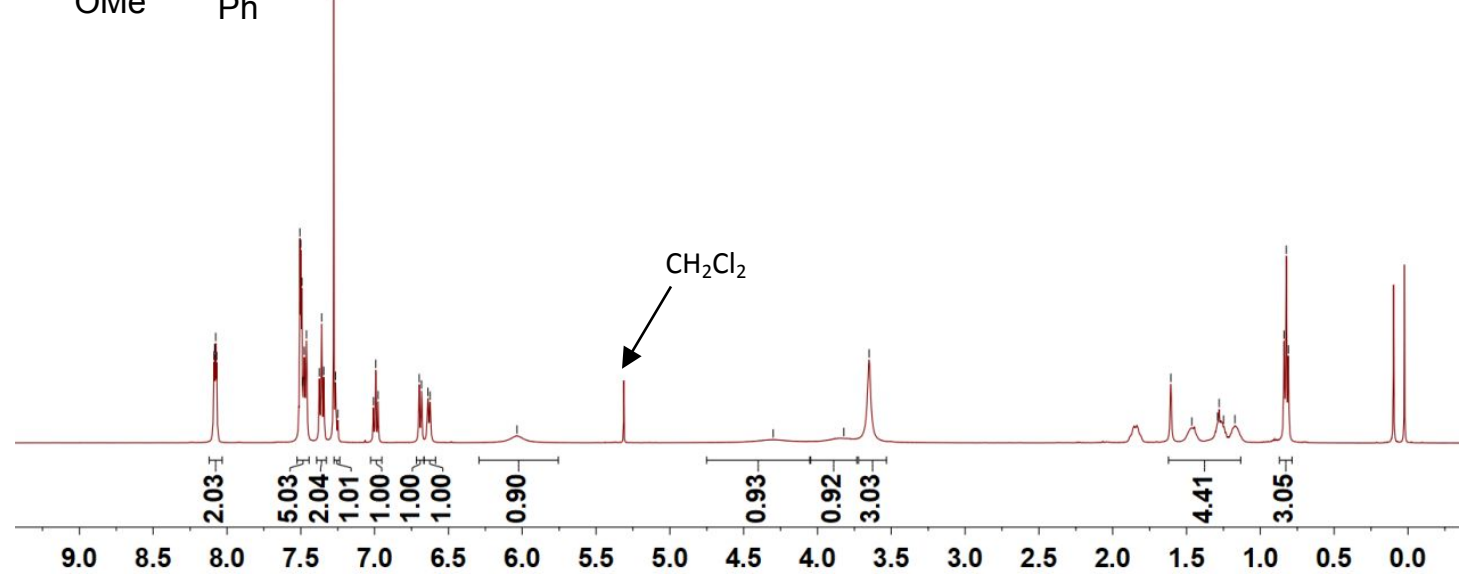

${ }^{13} \mathrm{C}$ NMR of compound 4am (126 $\mathrm{MHz}$ in $\left.\mathrm{CDCl}_{3}\right)$<smiles>[3H]/C=C1/Oc2c(OC)cccc2[C@@H](c2ccccc2)/C(c2ccccc2)=C/C(=O)C1CCC</smiles>

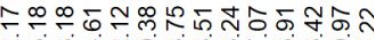

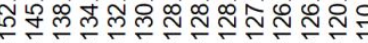

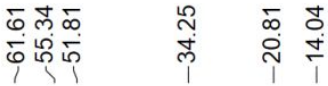

$\begin{array}{lllllllllllllllllllllll}220 & 210 & 200 & 190 & 180 & 170 & 160 & 150 & 140 & 130 & 120 & 110 & 100 & 90 & 80 & 70 & 60 & 50 & 40 & 30 & 20 & 10 & 0\end{array}$ 
${ }^{1} \mathrm{H}$ NMR of compound $4 \mathrm{an}\left(600 \mathrm{MHz}\right.$ in $\left.\mathrm{CDCl}_{3}\right)$

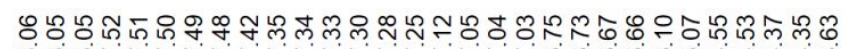

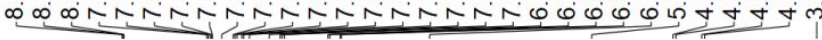<smiles>COc1cccc2c1OC(c1ccccc1)CC(=O)C(OCc1ccccc1)C2c1ccccc1</smiles>

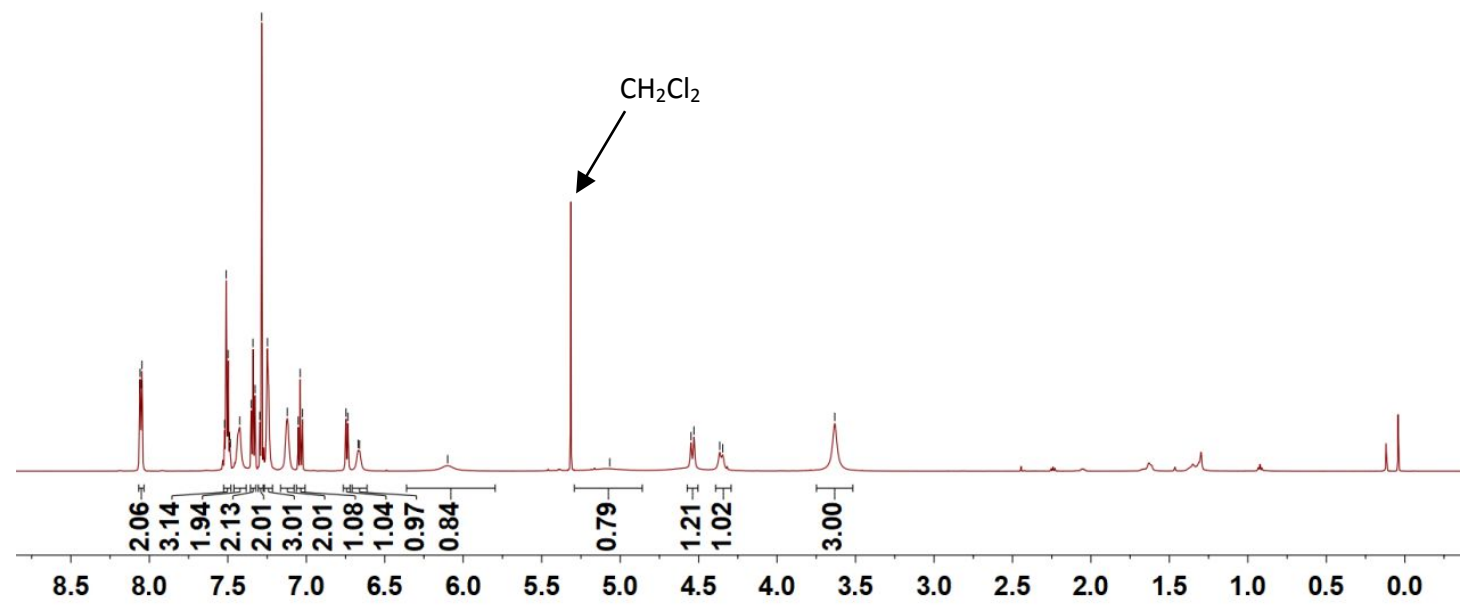

${ }^{13} \mathrm{C}$ NMR of compound 4an (126 $\mathrm{MHz}$ in $\left.\mathrm{CDCl}_{3}\right)$<smiles>COc1cccc2c1OC(c1ccccc1)C(OCc1ccccc1)C(=O)C2</smiles>

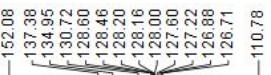


${ }^{1} \mathrm{H}$ NMR of compound $4 \mathrm{bn}\left(400 \mathrm{MHz}\right.$ in $\left.\mathrm{CDCl}_{3}\right)$

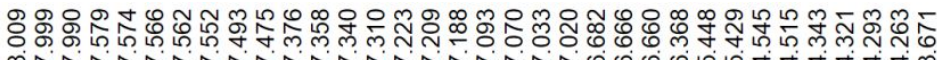

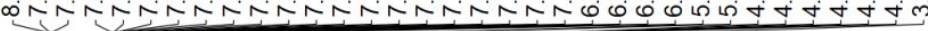<smiles>COc1ccc2c(c1)O/C(c1ccccc1)=C\C(=O)C(Cc1ccccc1)C2c1ccccc1</smiles>

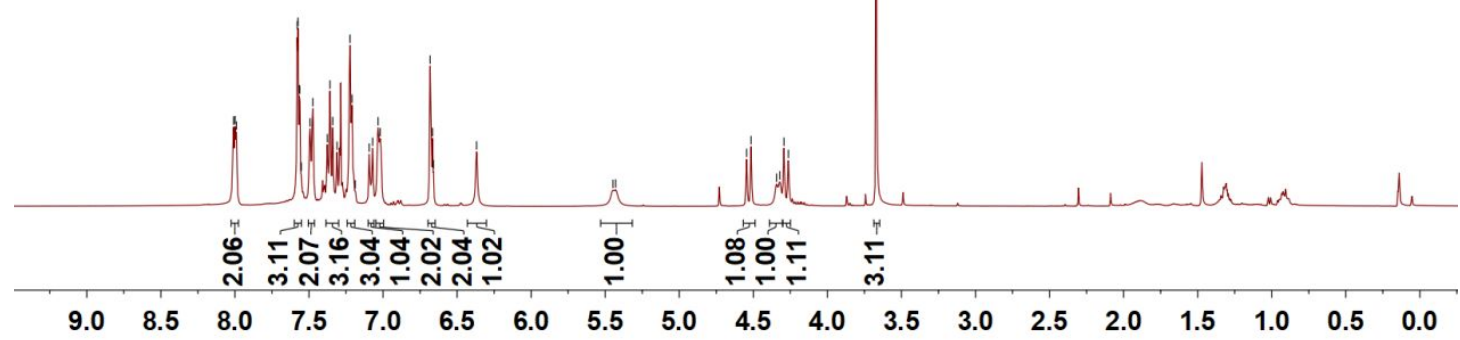

${ }^{13} \mathrm{C}$ NMR of compound $4 \mathrm{bn}\left(100 \mathrm{MHz}\right.$ in $\left.\mathrm{CDCl}_{3}\right)$

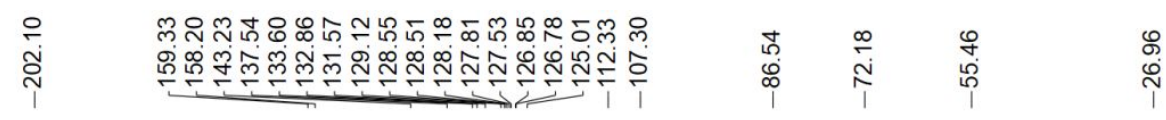<smiles>COc1ccc2c(c1)O/C(c1ccccc1)=C\C(=O)C(Cc1ccccc1)C2c1ccccc1</smiles>

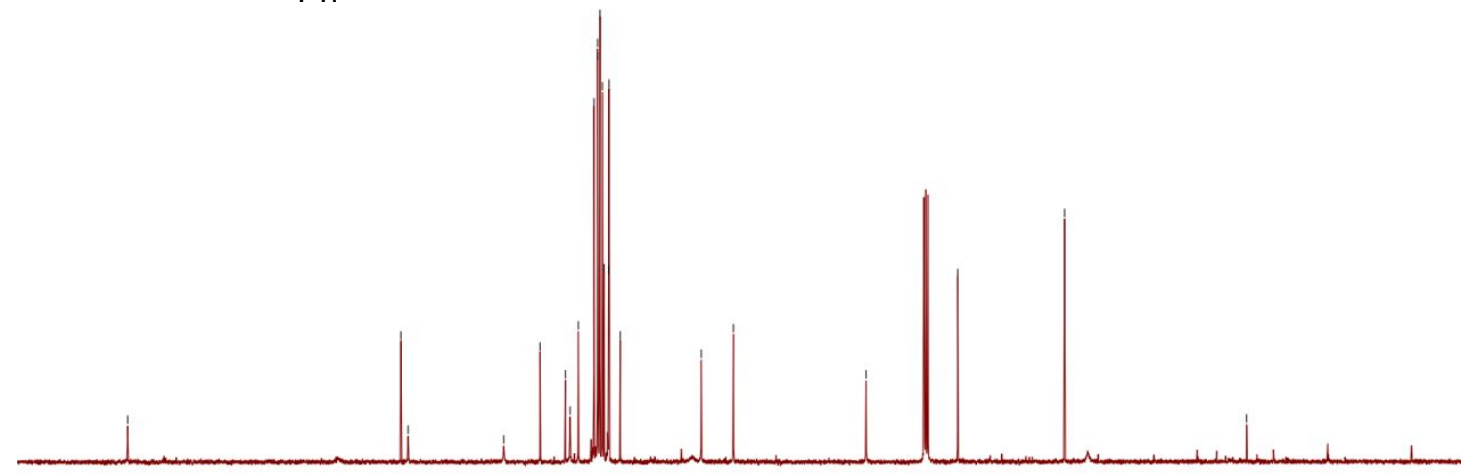

$\begin{array}{llllllllllllllllllllll}210 & 200 & 190 & 180 & 170 & 160 & 150 & 140 & 130 & 120 & 110 & 100 & 90 & 80 & 70 & 60 & 50 & 40 & 30 & 20 & 10 & 0\end{array}$ 
${ }^{1} \mathrm{H}$ NMR of compound 4gn (600 $\mathrm{MHz}$ in $\left.\mathrm{CDCl}_{3}\right)$

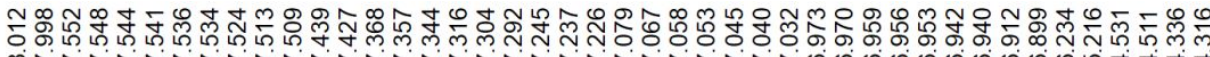

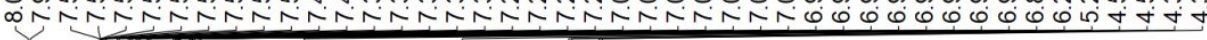<smiles>O=C1/C=C(\c2ccccc2)C(OCc2ccccc2)C(c2ccccc2)c2cccc(F)c2O1</smiles>

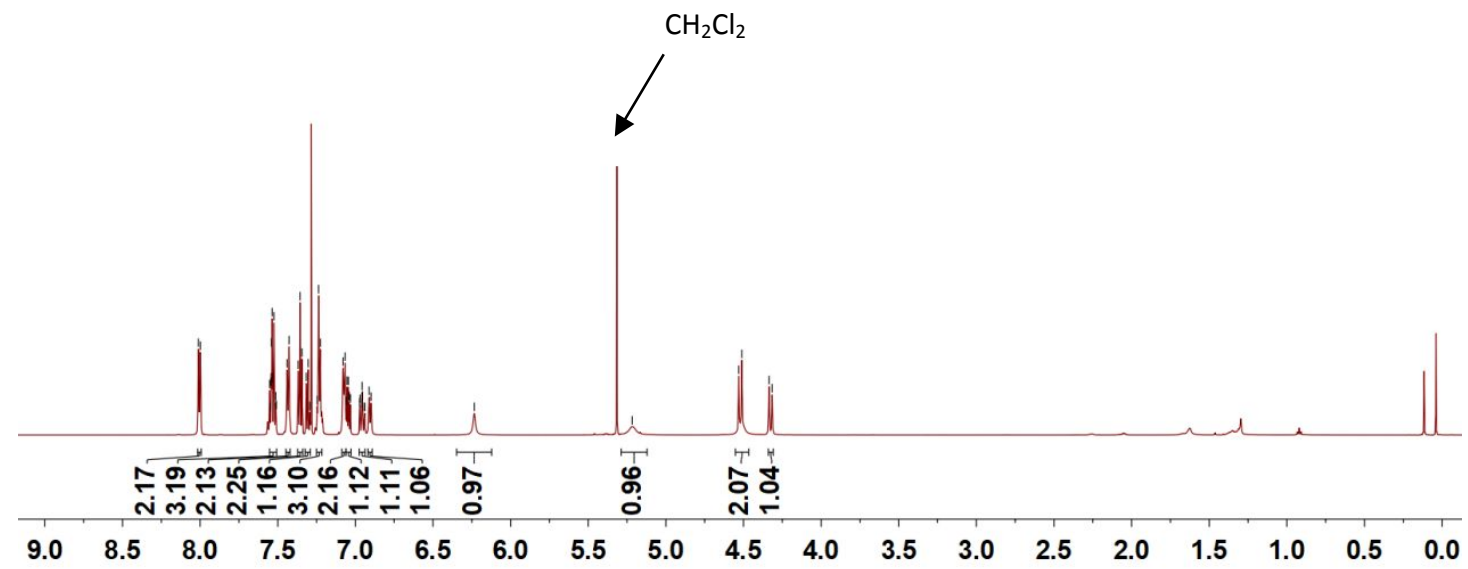

${ }^{13} \mathrm{C}$ NMR of compound 4gn (151 $\mathrm{MHz}$ in $\left.\mathrm{CDCl}_{3}\right)$

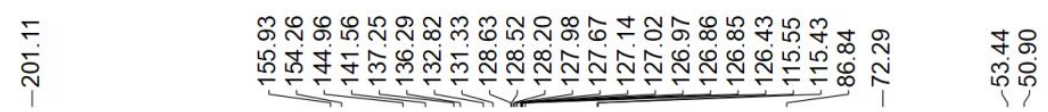<smiles>O=C1/C=C(\c2ccccc2)C(O)C(c2ccccc2)C(=O)Oc2c(F)cccc21</smiles>

$\begin{array}{llllllllllllllllllllll}210 & 200 & 190 & 180 & 170 & 160 & 150 & 140 & 130 & 120 & 110 & 100 & 90 & 80 & 70 & 60 & 50 & 40 & 30 & 20 & 10 & 0\end{array}$ 
${ }^{1} \mathrm{H}$ NMR of compound $4 \mathrm{ao}\left(500 \mathrm{MHz}\right.$ in $\left.\mathrm{CDCl}_{3}\right)$
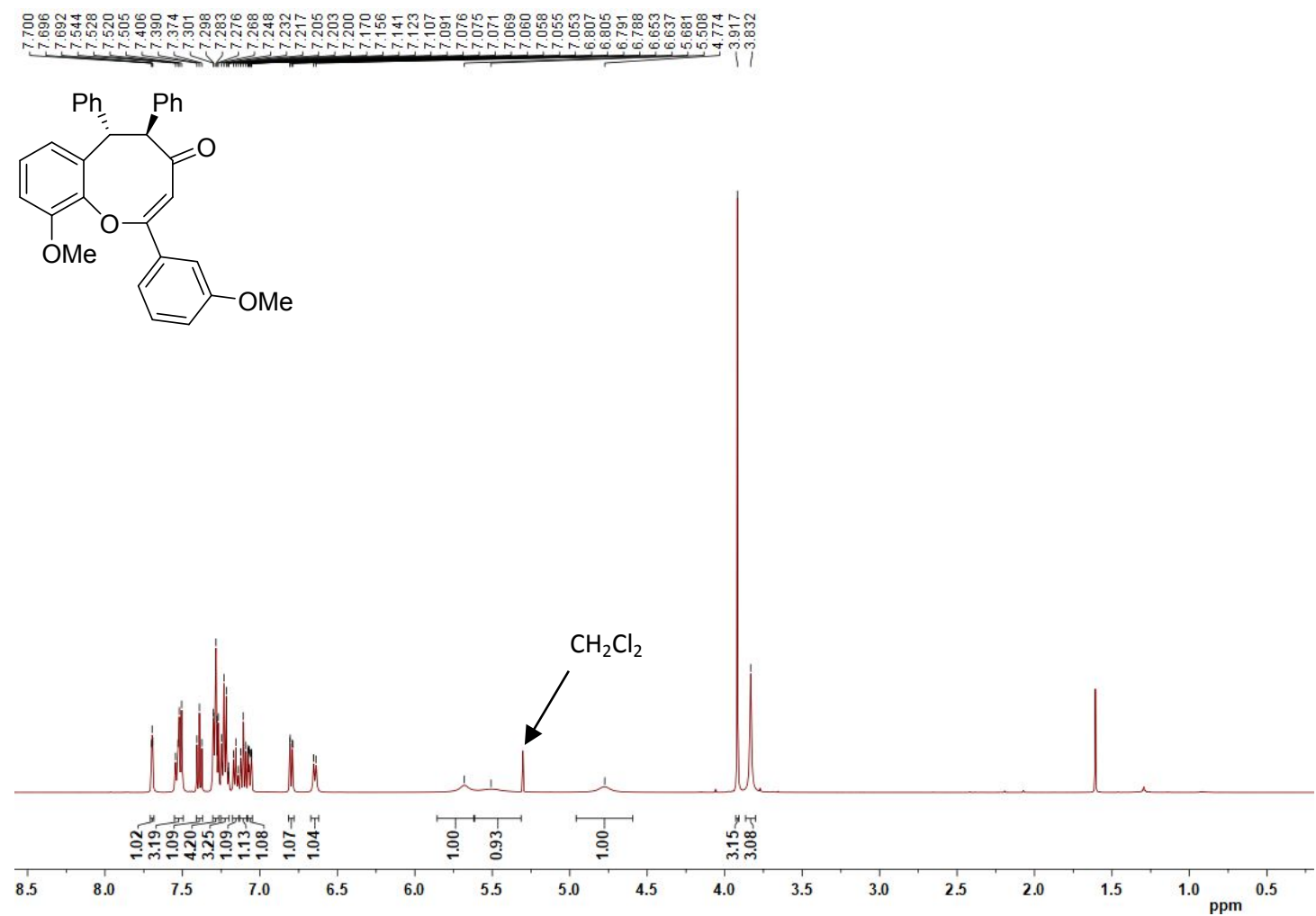

${ }^{13} \mathrm{C}$ NMR of compound $4 \mathrm{ao}\left(126 \mathrm{MHz}\right.$ in $\left.\mathrm{CDCl}_{3}\right)$
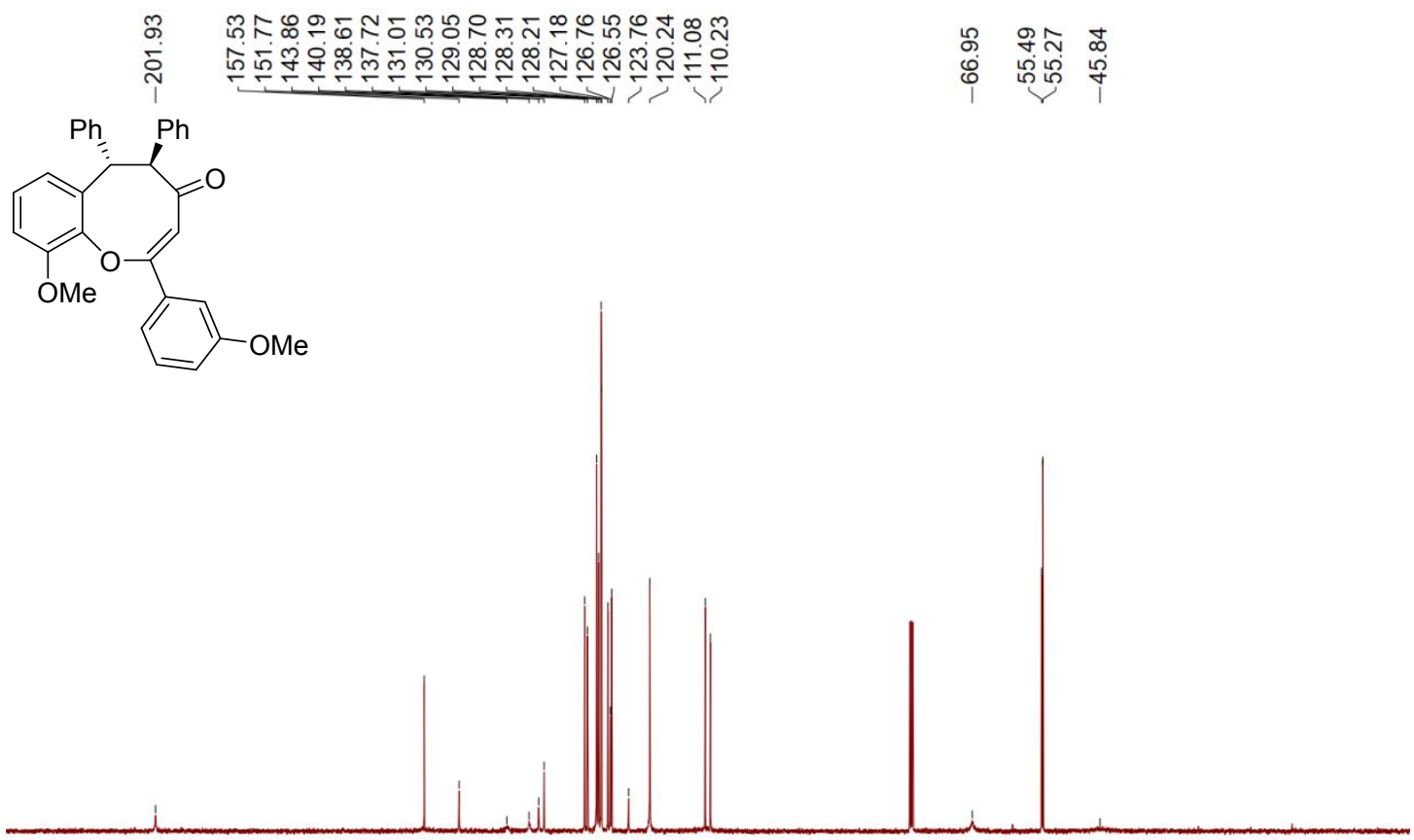

$\begin{array}{lllllllllllllllllllllll}220 & 210 & 200 & 190 & 180 & 170 & 160 & 150 & 140 & 130 & 120 & 110 & 100 & 90 & 80 & 70 & 60 & 50 & 40 & 30 & 20 & 10 & 0\end{array}$ 
${ }^{1} \mathrm{H}$ NMR of compound 4ap (500 $\mathrm{MHz}$ in $\left.\mathrm{CDCl}_{3}\right)$

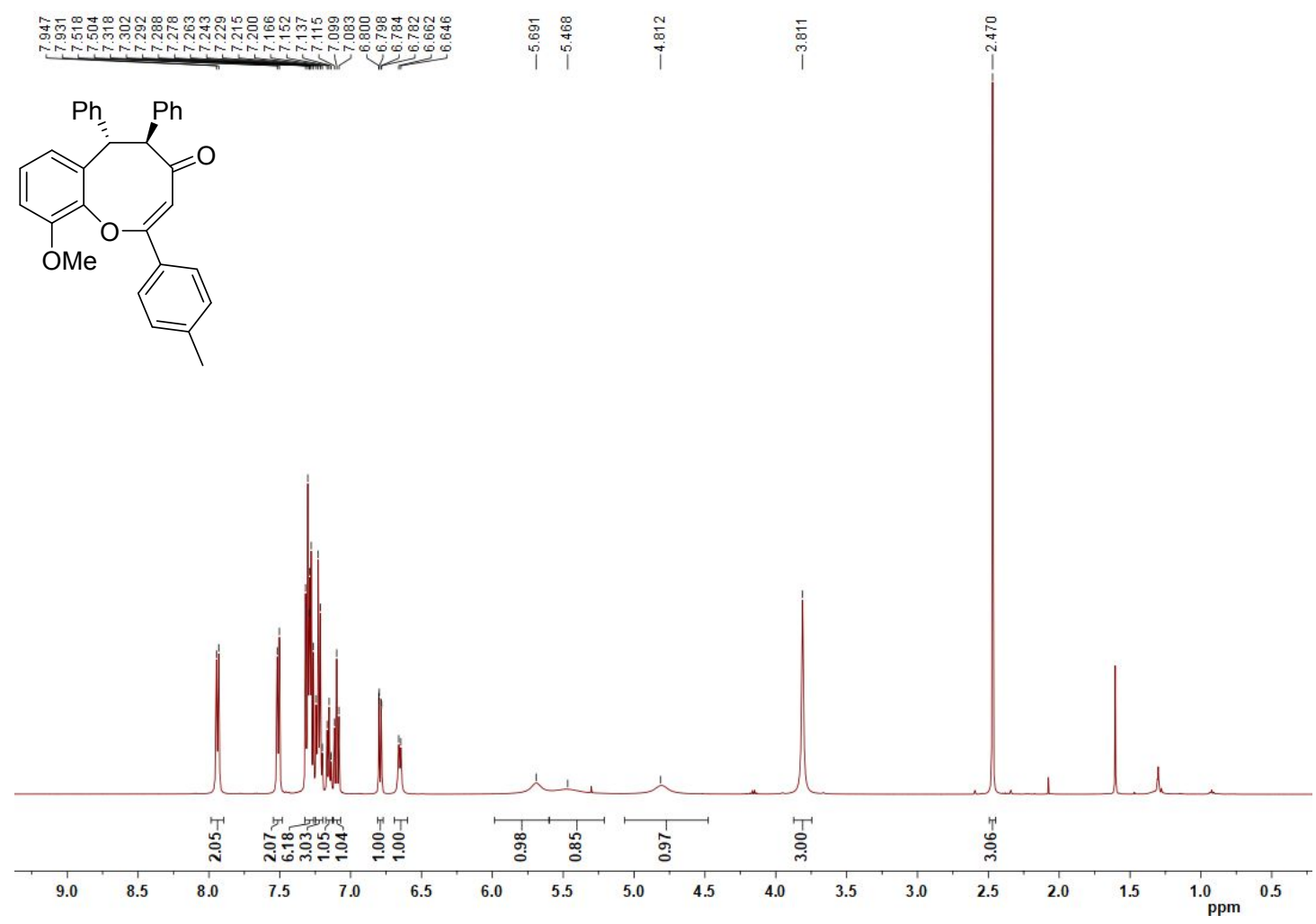

${ }^{13} \mathrm{C}$ NMR of compound $4 \mathrm{ap}\left(126 \mathrm{MHz}\right.$ in $\left.\mathrm{CDCl}_{3}\right)$

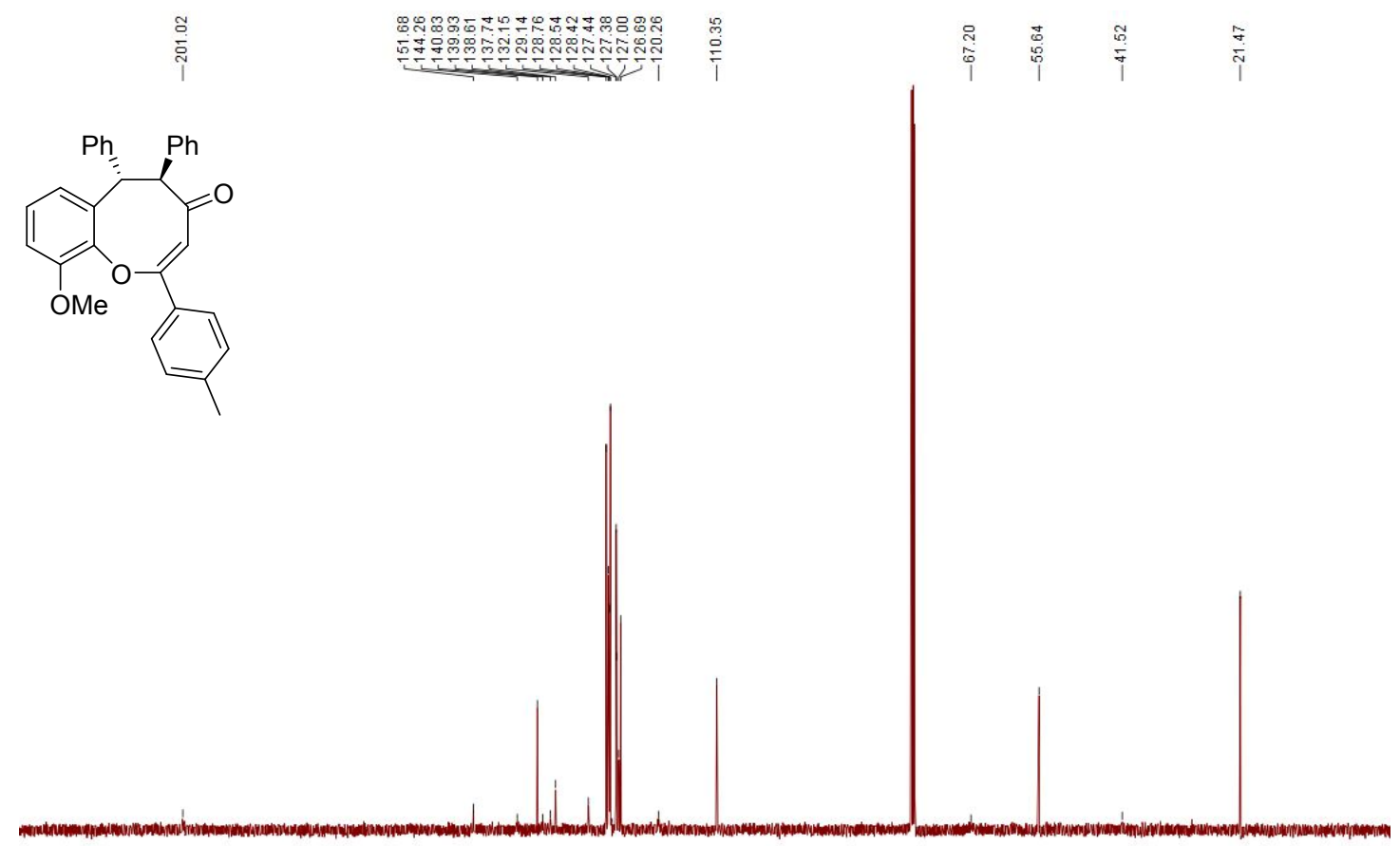

$\begin{array}{llllllllllllllllllllllllllllll}1 & 220 & 210 & 200 & 190 & 180 & 170 & 160 & 150 & 140 & 130 & 120 & 110 & 100 & 90 & 80 & 70 & 60 & 50 & 40 & 30 & 20 & 10 & 0\end{array}$ 
${ }^{1} \mathrm{H}$ NMR of compound $4 \mathrm{aq}\left(500 \mathrm{MHz}\right.$ in $\left.\mathrm{CDCl}_{3}\right)$
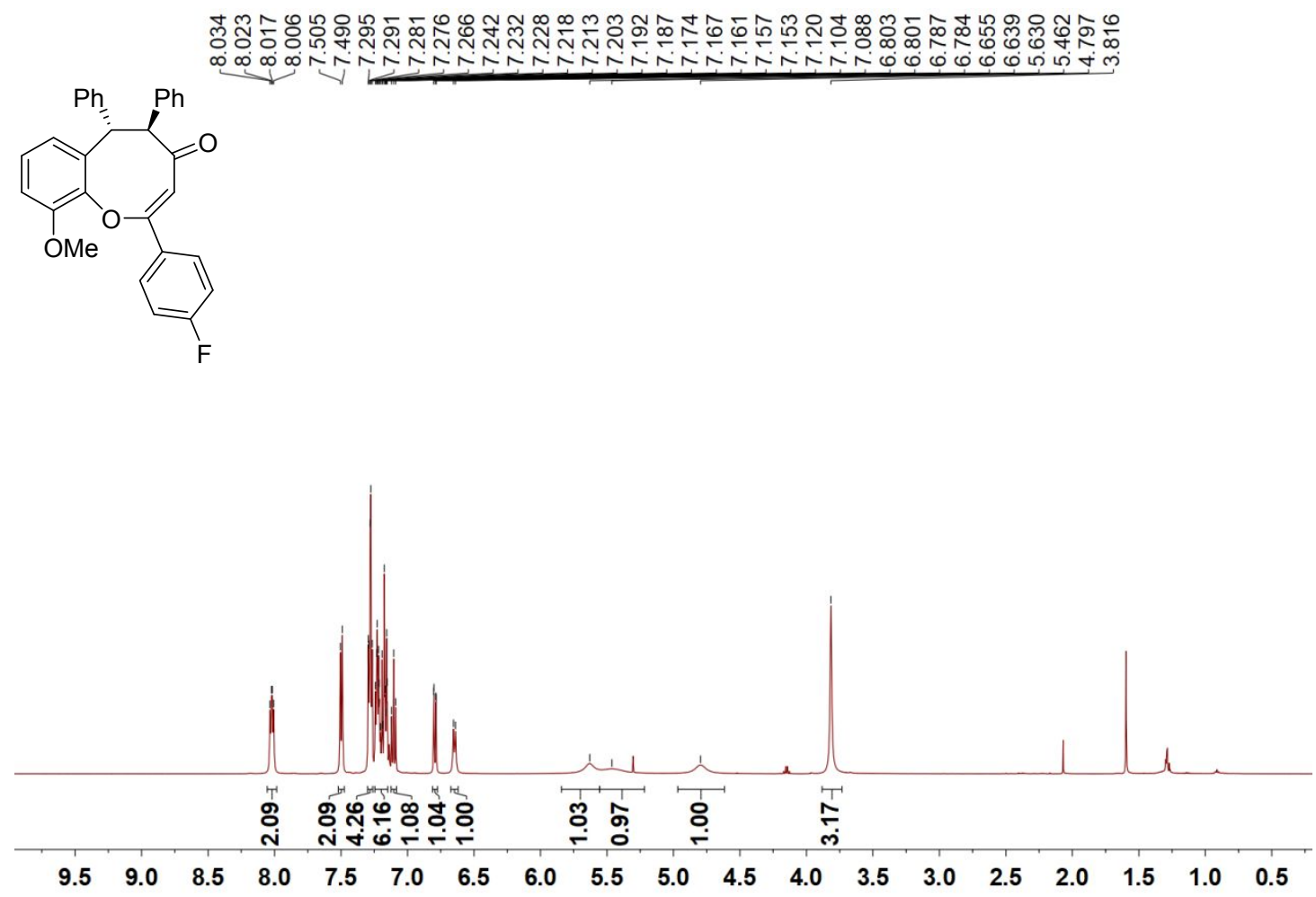

${ }^{13} \mathrm{C}$ NMR of compound 4aq (126 $\mathrm{MHz}$ in $\left.\mathrm{CDCl}_{3}\right)$

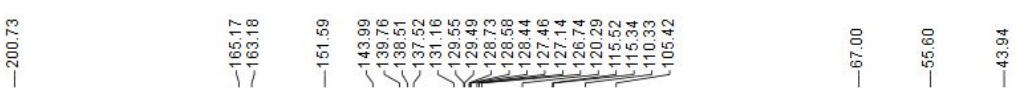<smiles>COc1cccc2c1OC(c1ccc(F)cc1)C(=O)C(c1ccccc1)C2=O</smiles>

\begin{tabular}{lllllllllllllllllllllllllllllllllll}
\hline 220 & 210 & 200 & 190 & 180 & 170 & 160 & 150 & 140 & 130 & 120 & 110 & 100 & 90 & 80 & 70 & 60 & 50 & 40 & 30 & 20 & 10 & 0
\end{tabular} 
${ }^{1} \mathrm{H}$ NMR of compound $4 \mathrm{ar}\left(500 \mathrm{MHz}\right.$ in $\left.\mathrm{CDCl}_{3}\right)$

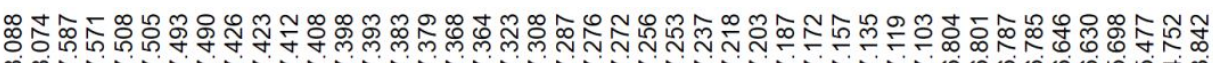

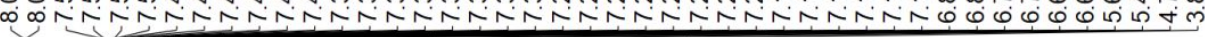<smiles>COc1cccc2c1OC(c1ccccc1Cl)C(=O)C(c1ccccc1)C2=O</smiles>

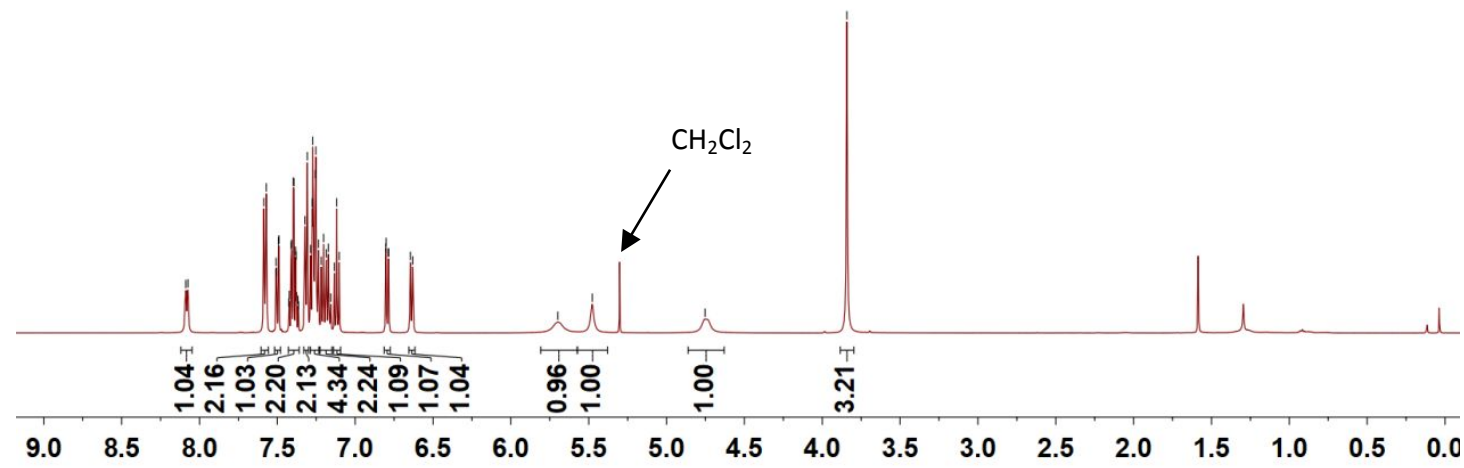

${ }^{13} \mathrm{C}$ NMR of compound 4ar (126 $\mathrm{MHz}$ in $\left.\mathrm{CDCl}_{3}\right)$

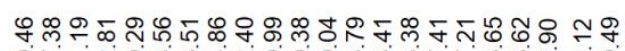

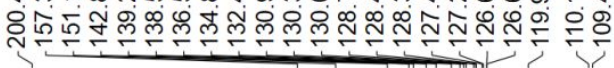

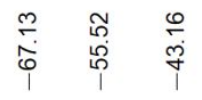<smiles>COc1cccc2c1OC(c1ccccc1Cl)C=C(C(=O)C(c1ccccc1)c1ccccc1)C2</smiles>

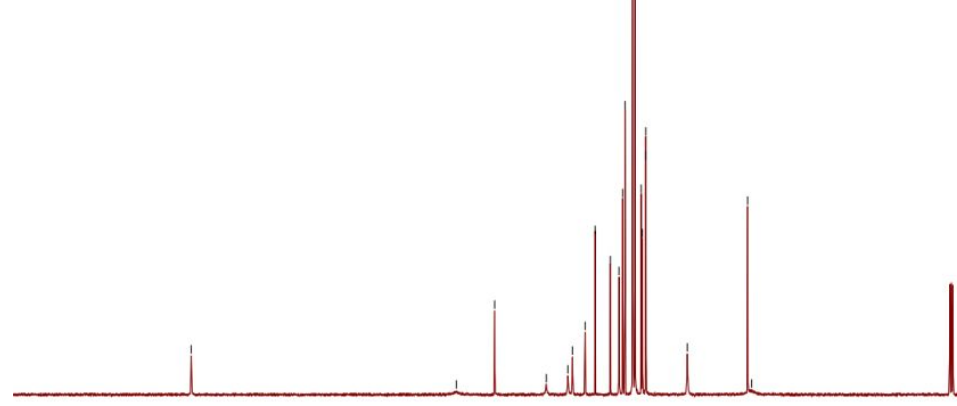

$\begin{array}{lllllllllllllllllllllll}220 & 210 & 200 & 190 & 180 & 170 & 160 & 150 & 140 & 130 & 120 & 110 & 100 & 90 & 80 & 70 & 60 & 50 & 40 & 30 & 20 & 10 & 0\end{array}$ 
${ }^{1} \mathrm{H}$ NMR of compound 4as (500 $\mathrm{MHz}$ in $\left.\mathrm{CDCl}_{3}\right)$

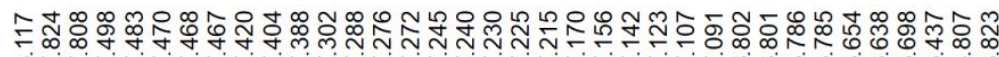

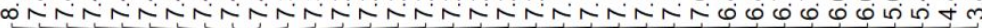<smiles>COc1cccc2c1OC(c1cccc(Cl)c1)C=C(C(=O)C(c1ccccc1)c1ccccc1)C2</smiles>

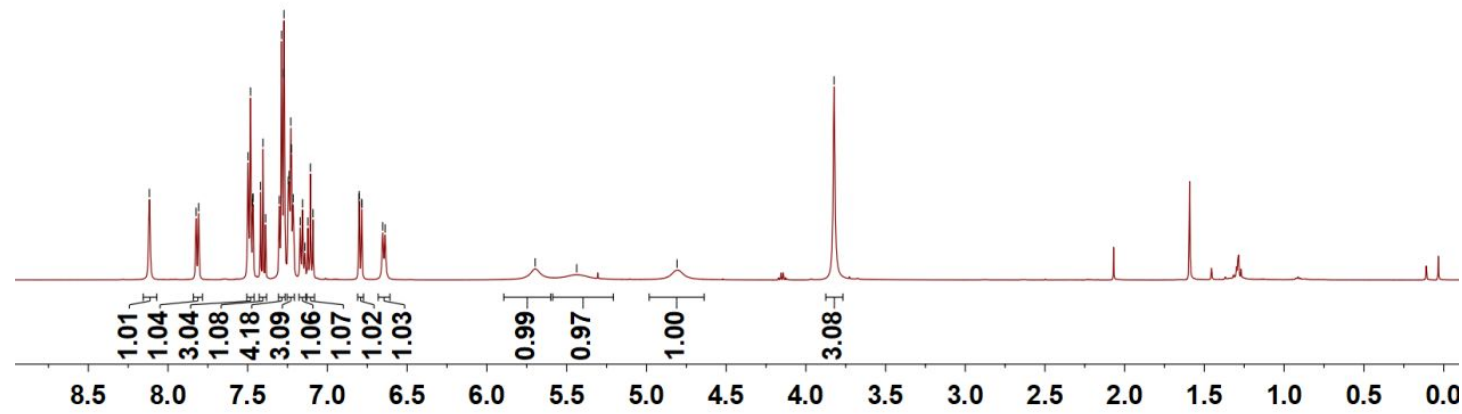

${ }^{13} \mathrm{C}$ NMR of compound 4as (126 $\mathrm{MHz}$ in $\left.\mathrm{CDCl}_{3}\right)$

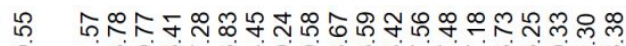

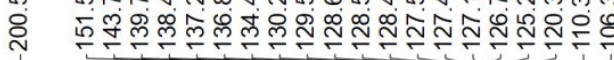

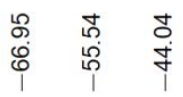<smiles>COc1cccc2c1OC(c1cccc(Cl)c1)C=C(C(=O)C(c1ccccc1)c1ccccc1)C2</smiles>

$-\mathrm{Cl}$

$\begin{array}{lllllllllllllllllllllll}220 & 210 & 200 & 190 & 180 & 170 & 160 & 150 & 140 & 130 & 120 & 110 & 100 & 90 & 80 & 70 & 60 & 50 & 40 & 30 & 20 & 10 & 0\end{array}$ 
${ }^{1} \mathrm{H}$ NMR of compound 4at (500 $\mathrm{MHz}$ in $\mathrm{CDCl}_{3}$ )

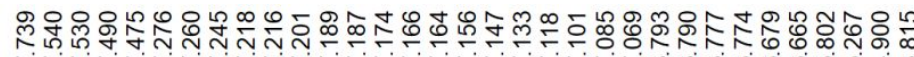

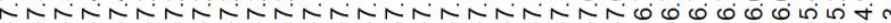<smiles>COc1cccc2c1OC(c1cccs1)C(=O)C(c1ccccc1)C(=O)C2=O</smiles>

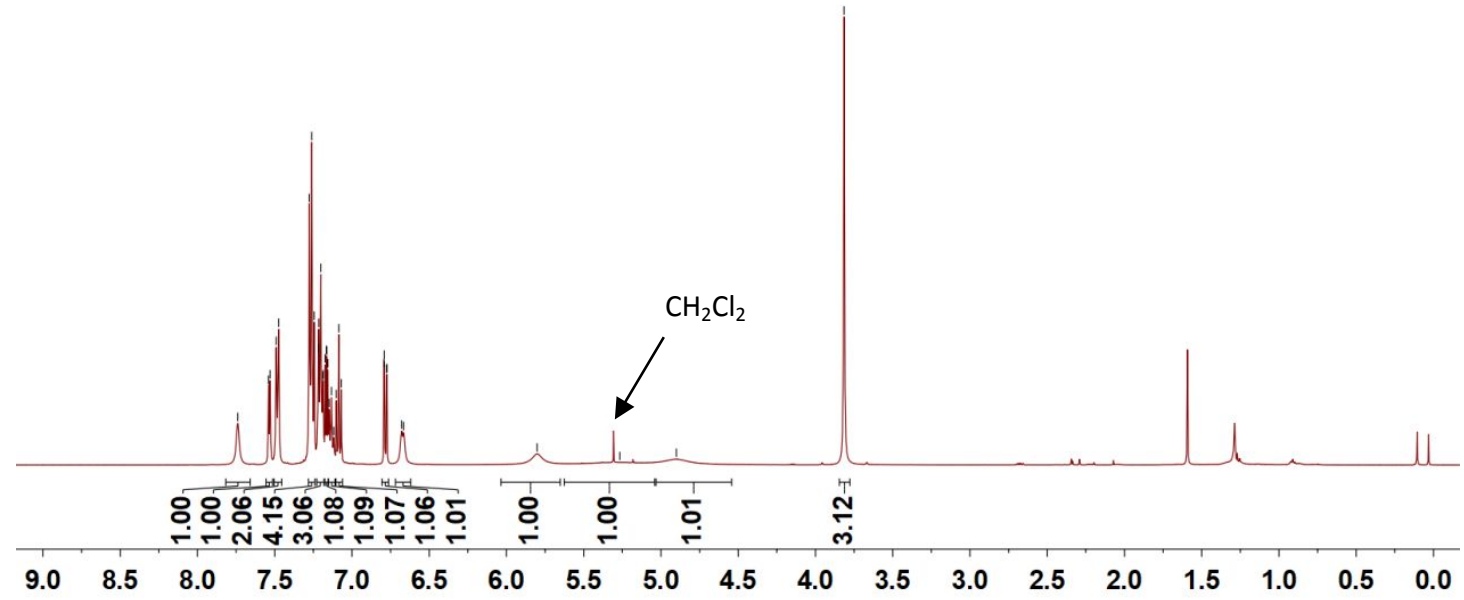

${ }^{13} \mathrm{C}$ NMR of compound 4at (126 $\mathrm{MHz}$ in $\left.\mathrm{CDCl}_{3}\right)$

ণ্ণ

किष्ञ

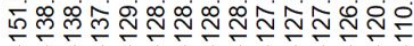

충ำ<smiles>COc1cccc2c1O/C(c1cccs1)=C\C(=O)C(c1ccccc1)C2c1ccccc1</smiles>

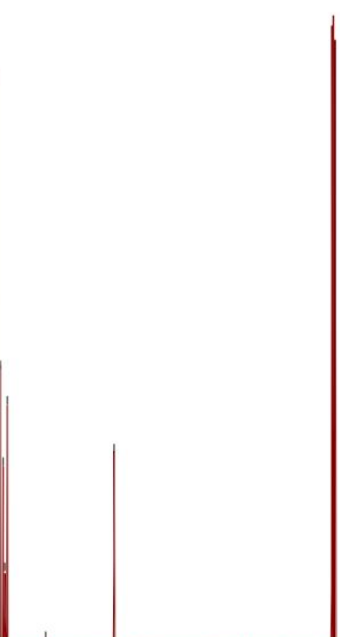

$\begin{array}{llllllllllllllllllllll}210 & 200 & 190 & 180 & 170 & 160 & 150 & 140 & 130 & 120 & 110 & 100 & 90 & 80 & 70 & 60 & 50 & 40 & 30 & 20 & 10 & 0\end{array}$ 
${ }^{1} \mathrm{H}$ NMR of compound 4au (500 $\mathrm{MHz}$ in $\mathrm{CDCl}_{3}$ )

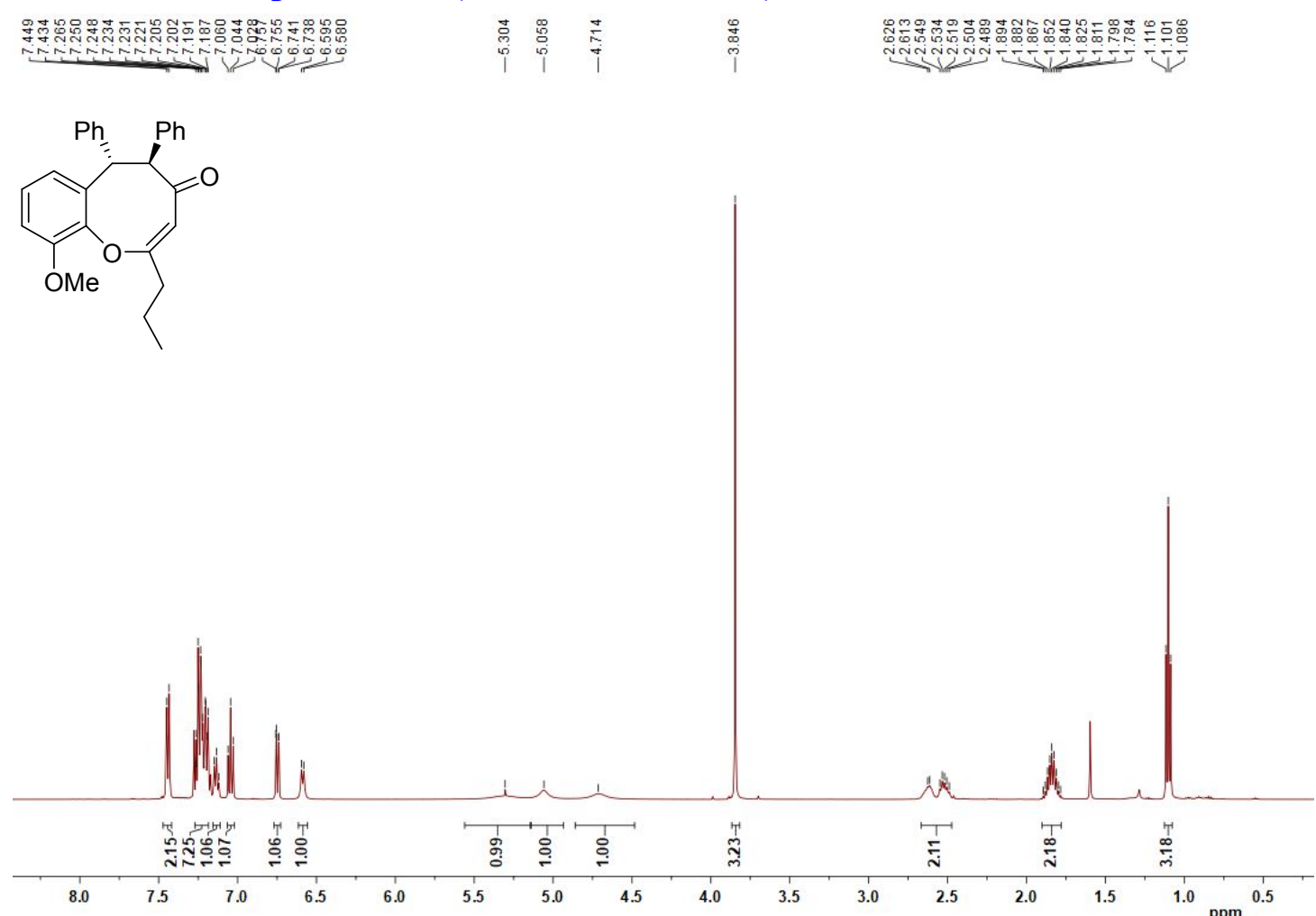

${ }^{13} \mathrm{C}$ NMR of compound 4au (126 $\mathrm{MHz}$ in $\left.\mathrm{CDCl}_{3}\right)$

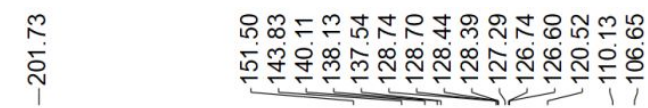

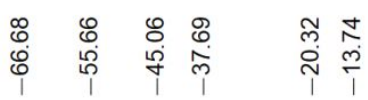<smiles>CCCC1=CC(=O)C(c2ccccc2)C(c2ccccc2)c2cccc(OC)c2O1</smiles>

$\begin{array}{lllllllllllllllllllllll}220 & 210 & 200 & 190 & 180 & 170 & 160 & 150 & 140 & 130 & 120 & 110 & 100 & 90 & 80 & 70 & 60 & 50 & 40 & 30 & 20 & 10 & 0\end{array}$ 
${ }^{1} \mathrm{H}$ NMR of compound $4 \mathrm{av}\left(500 \mathrm{MHz}\right.$ in $\left.\mathrm{CDCl}_{3}\right)$

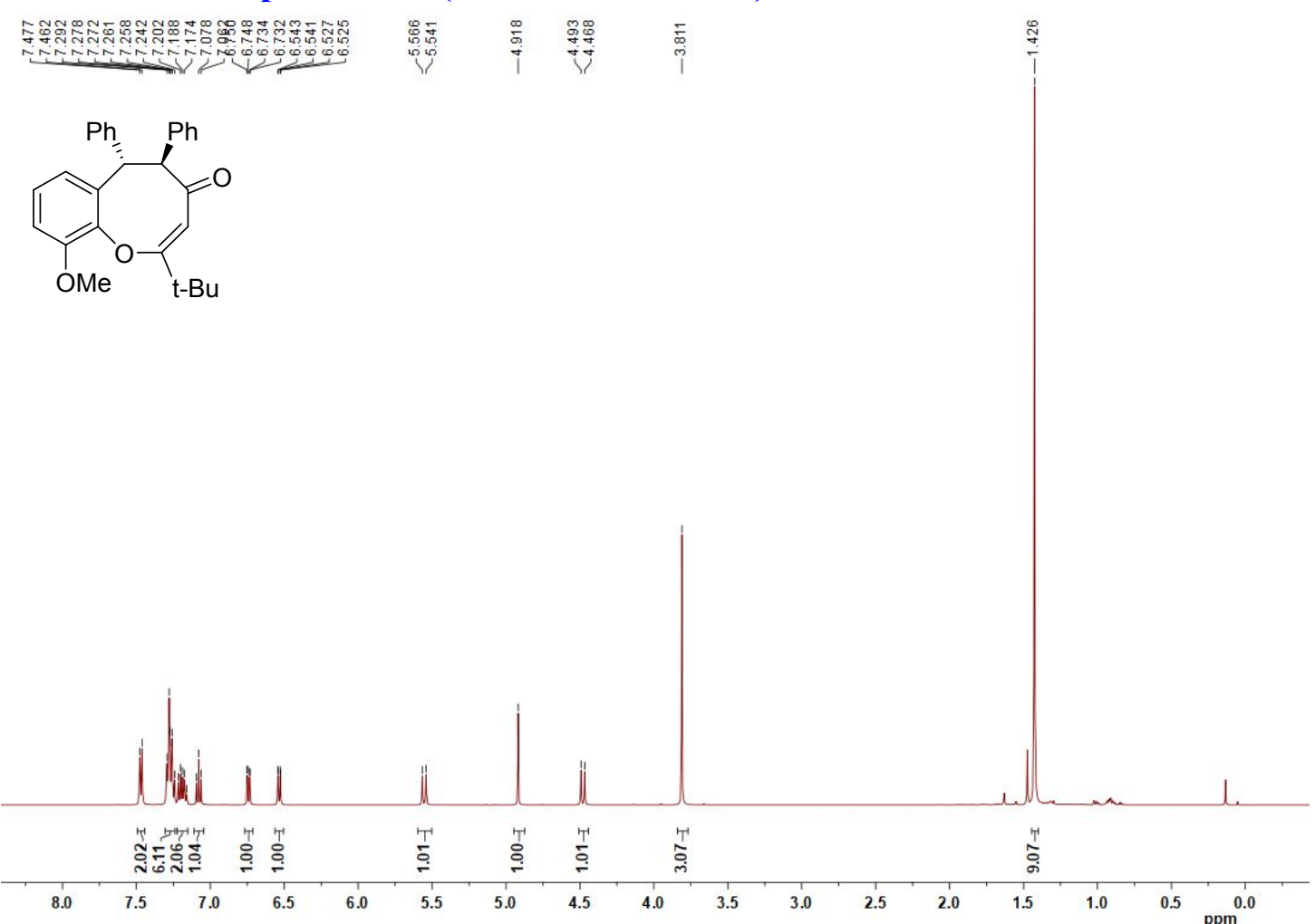

${ }^{13} \mathrm{C}$ NMR of compound $4 \mathrm{av}\left(126 \mathrm{MHz}\right.$ in $\left.\mathrm{CDCl}_{3}\right)$

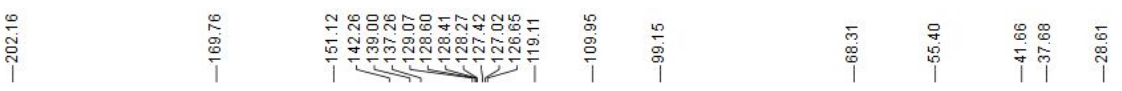<smiles>COc1cccc2c1OC(C(C)(C)C)CC(=O)C(c1ccccc1)C2c1ccccc1</smiles>

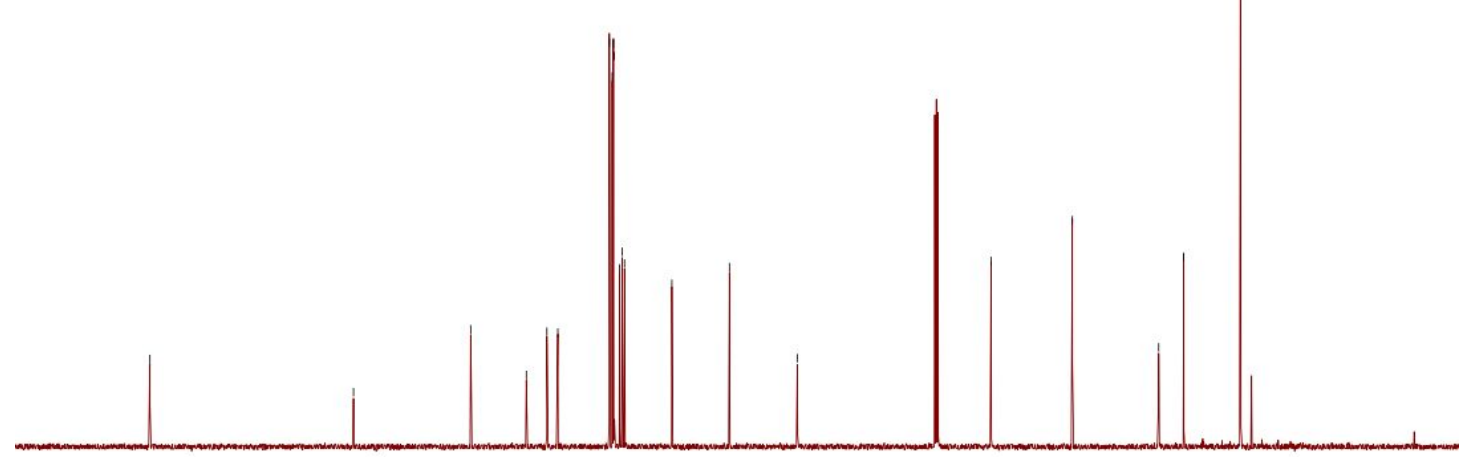

\begin{tabular}{llllllllllllllllllllllllllll}
\hline 220 & 210 & 200 & 190 & 180 & 170 & 160 & 150 & 140 & 130 & 120 & 110 & 100 & 90 & 80 & 70 & 60 & 50 & 40 & 30 & 20 & 10 & 0
\end{tabular} 
${ }^{1} \mathrm{H}$ NMR of compound $4 \mathrm{gv}\left(600 \mathrm{MHz}\right.$ in $\left.\mathrm{CDCl}_{3}\right)$

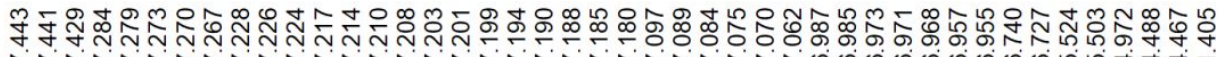

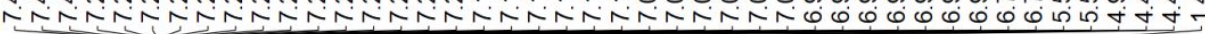<smiles>CC(C)(C)C1=CC(=O)C(c2ccccc2)C(c2ccccc2)c2cccc(F)c2O1</smiles>

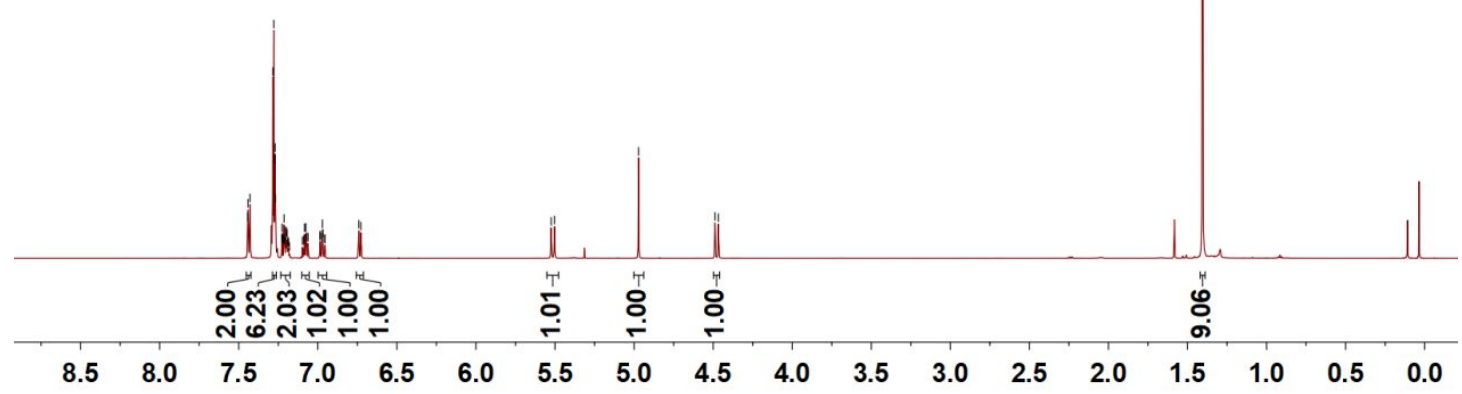

${ }^{13} \mathrm{C}$ NMR of compound $4 \mathrm{gv}\left(151 \mathrm{MHz}\right.$ in $\left.\mathrm{CDCl}_{3}\right)$

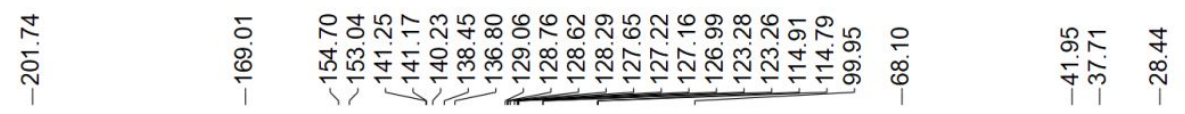<smiles>CC(C)(C)C1=CC(=O)C(c2ccccc2)C(c2ccccc2)c2cccc(F)c2O1</smiles> 
${ }^{1} \mathrm{H}$ NMR of compound $5\left(500 \mathrm{MHz}\right.$ in $\left.\mathrm{CDCl}_{3}\right)$

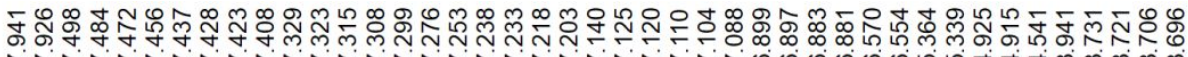

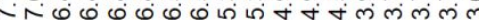<smiles>COc1cccc2c1O/C(c1ccccc1)=C\C(O)C(c1ccccc1)C2c1ccccc1</smiles>

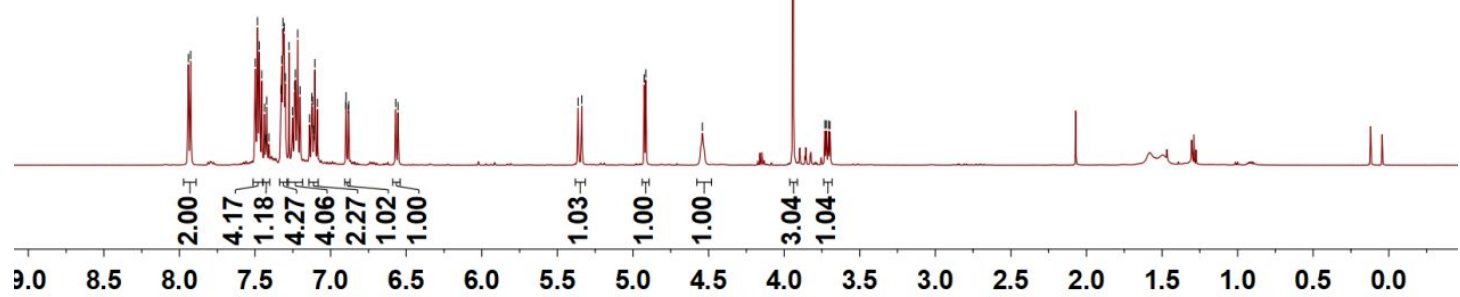

${ }^{13} \mathrm{C}$ NMR of compound 5 (126 $\mathrm{MHz}$ in $\left.\mathrm{CDCl}_{3}\right)$

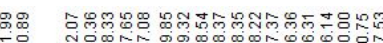

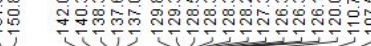<smiles>COc1cccc2c1O/C(c1ccccc1)=C\C(O)C(c1ccccc1)C2c1ccccc1</smiles>

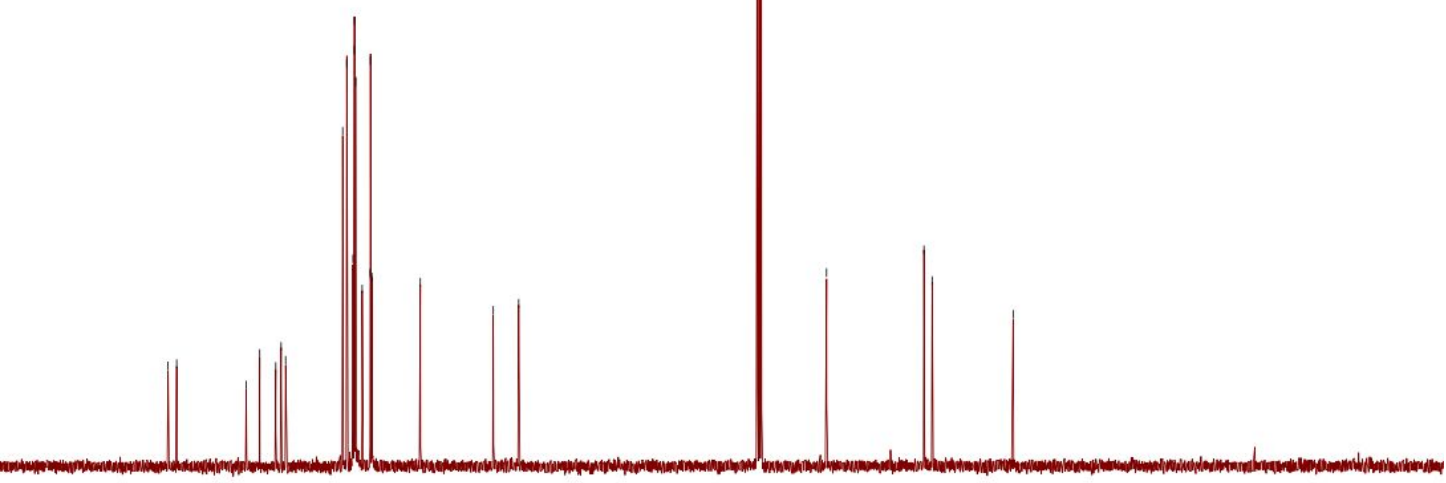

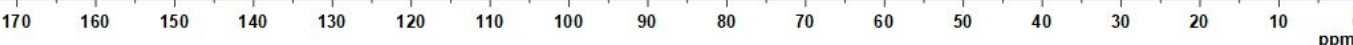


${ }^{1} \mathrm{H}$ NMR of compound $6\left(500 \mathrm{MHz}\right.$ in $\left.\mathrm{CDCl}_{3}\right)$

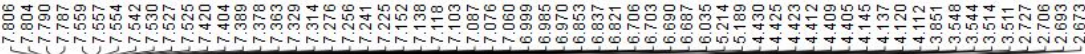<smiles>COc1cccc(C(c2ccccc2)C(O)CC(=O)c2ccccc2)c1O</smiles>
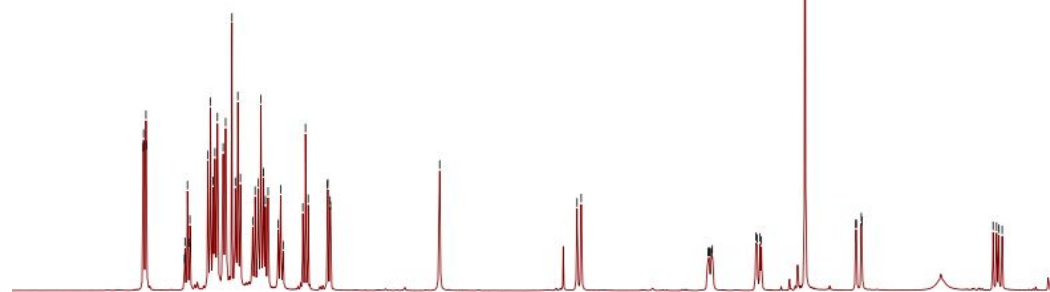

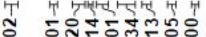

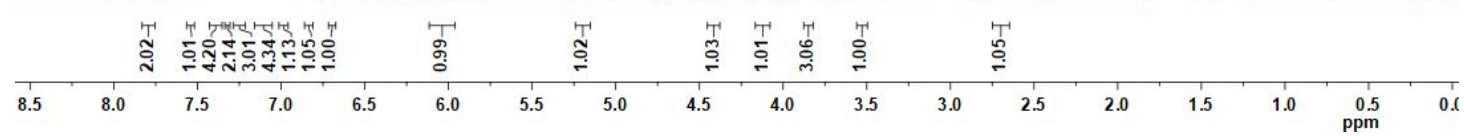

${ }^{13} \mathrm{C}$ NMR of compound $6\left(126 \mathrm{MHz}\right.$ in $\left.\mathrm{CDCl}_{3}\right)$<smiles>COc1cccc([C@H](c2ccccc2)[C@H](O)CC(=O)c2ccccc2)c1O</smiles>

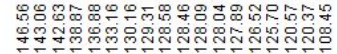

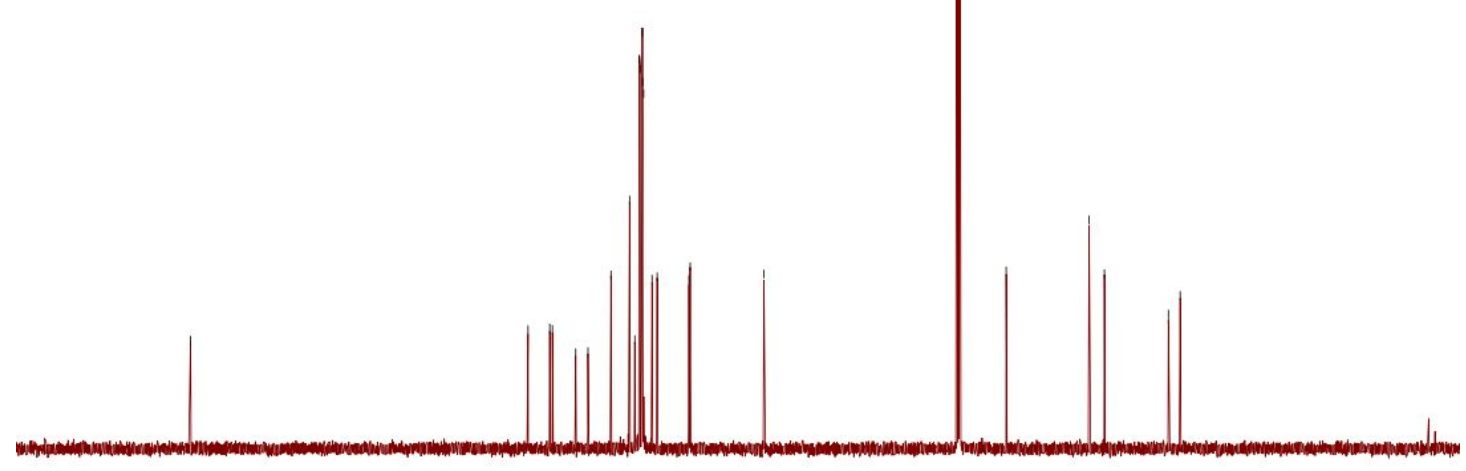

$\begin{array}{llllllllllllllllllllllllll}220 & 210 & 200 & 190 & 180 & 170 & 160 & 150 & 140 & 130 & 120 & 110 & 100 & 90 & 80 & 70 & 60 & 50 & 40 & 30 & 20 & 10 & 0 \\ \mathrm{ppm} & \end{array}$ 
${ }^{1} \mathrm{H}$ NMR of compound D-4aa (500 $\mathrm{MHz}$ in $\mathrm{CDCl}_{3}$ )

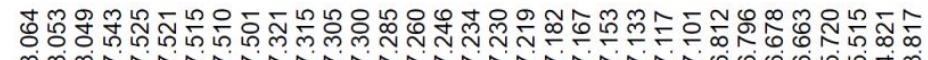

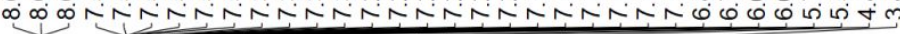
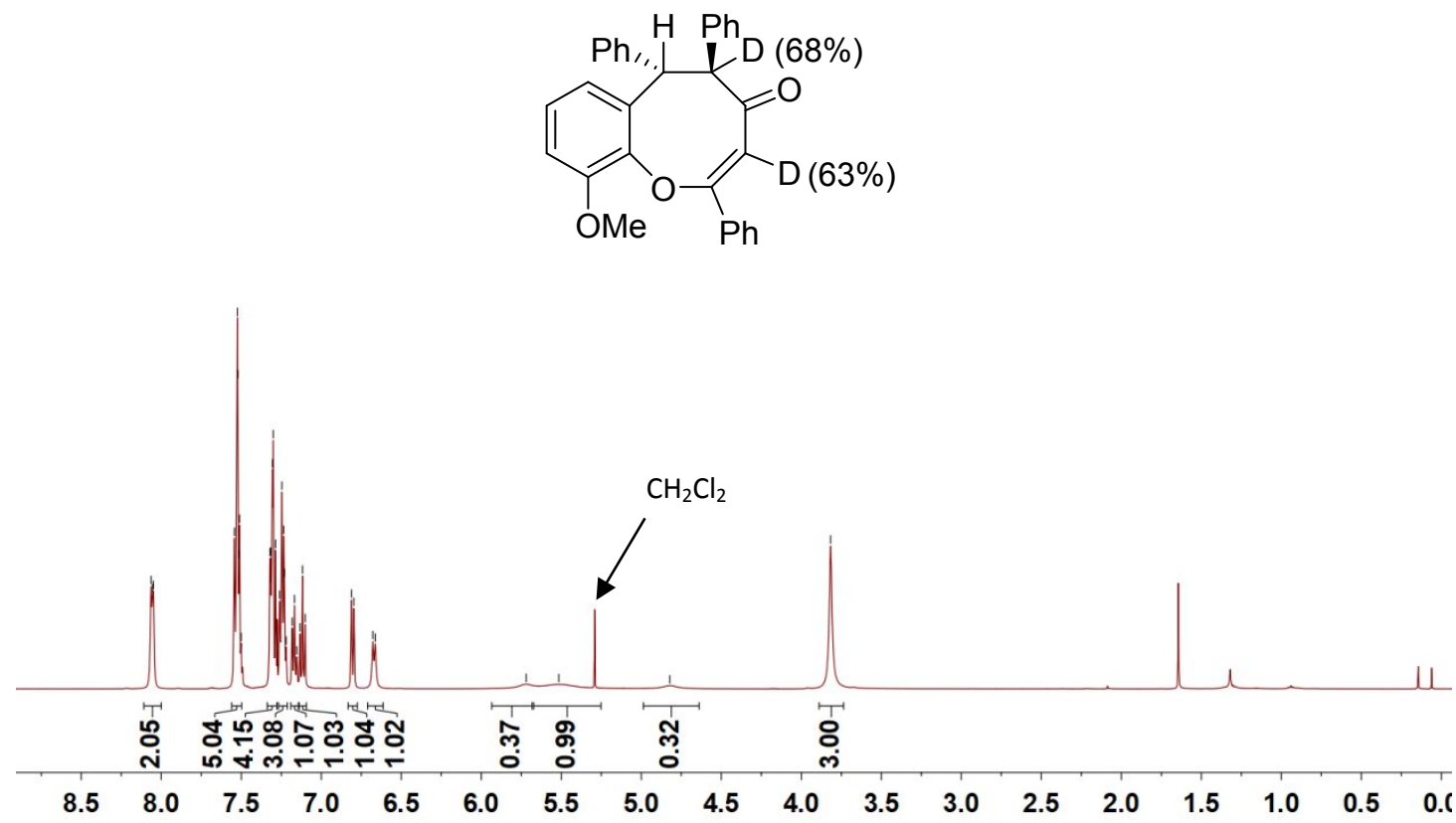


\section{Crystal data and structure refinement for enantiopure 4as and 5.}

Crystals of enantiopure 4as suitable for X-ray analysis were obtained from crystallization in a solution of $\mathrm{CH}_{2} \mathrm{Cl}_{2}$ and n-hexan
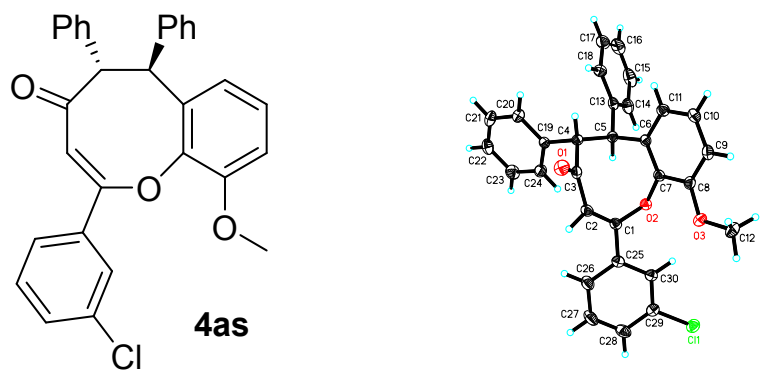

(ellipsoid contour at $50 \%$ probability level)

CCDC deposition number

Empirical formula

Formula weight

Temperature

Wavelength

Crystal system

Space group

Unit cell dimensions

Volume

Z

Density (calculated)

Absorption coefficient

$\mathrm{F}(000)$

Crystal size

Theta range for data collection

Index ranges

Reflections collected

Independent reflections

Completeness to theta $=25.242^{\circ}$

Absorption correction

Max. and min. transmission

Refinement method

Data / restraints / parameters

Goodness-of-fit on $\mathrm{F}^{2}$

Final $\mathrm{R}$ indices [I $>2 \operatorname{sigma(I)]}$

$\mathrm{R}$ indices (all data)

Absolute structure parameter
2012000

$\mathrm{C}_{30} \mathrm{H}_{23} \mathrm{Cl} \mathrm{O}_{3}$

466.93

193(2) K

$0.71073 \AA$

Tetragonal

$\mathrm{P}_{3}$

$\mathrm{a}=13.8111(3) \AA$

$\alpha=90^{\circ}$.

$\mathrm{b}=13.8111(3) \AA$

$\beta=90^{\circ}$.

$\mathrm{c}=12.2301(3) \AA$

$\gamma=90^{\circ}$

$2332.85(12) \AA^{3}$

4

$1.329 \mathrm{Mg} / \mathrm{m}^{3}$

$0.195 \mathrm{~mm}^{-1}$

976

$0.180 \times 0.150 \times 0.110 \mathrm{~mm}^{3}$

2.669 to $25.987^{\circ}$

$-17<=\mathrm{h}<=17,-17<=\mathrm{k}<=15,-14<=1<=15$

28747

$4564[\mathrm{R}(\mathrm{int})=0.0367]$

$99.7 \%$

Semi-empirical from equivalents

0.7456 and 0.6845

Full-matrix least-squares on $\mathrm{F}^{2}$

4564 / 1 / 308

1.069

$\mathrm{R} 1=0.0364, \mathrm{wR} 2=0.0864$

$\mathrm{R} 1=0.0419, \mathrm{wR} 2=0.0904$

$0.006(19)$ 
Extinction coefficient

Largest diff. peak and hole $\mathrm{n} / \mathrm{a}$

0.550 and -0.236 e. $\AA^{-3}$

Crystals of enantiopure 5 suitable for X-ray analysis were obtained from crystallization in a solution of $\mathrm{CH}_{2} \mathrm{Cl}_{2}$ and n-hexan<smiles>COc1cccc2c1O/C(c1ccccc1)=C\[C@@H](O)[C@H](c1ccccc1)[C@H]2c1ccccc1</smiles>

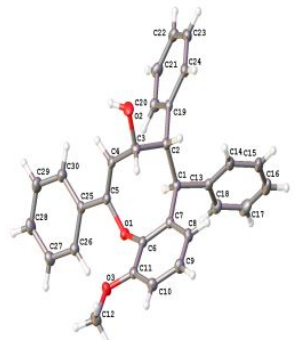

(ellipsoid contour at 50\% probability level)

CCDC deposition number

Empirical formula

Formula weight

Temperature

Wavelength

Crystal system

Space group

Unit cell dimensions

Volume

Z

Density (calculated)

Absorption coefficient

$\mathrm{F}(000)$

Crystal size

Theta range for data collection

Index ranges

Reflections collected

Independent reflections

Completeness to theta $=53.594^{\circ}$

Absorption correction

Max. and min. transmission

Refinement method

Data / restraints / parameters

Goodness-of-fit on $\mathrm{F}^{2}$

Final $\mathrm{R}$ indices [I $>2 \operatorname{sigma}(\mathrm{I})]$
2058104

$\mathrm{C}_{30} \mathrm{H}_{26} \mathrm{O}_{3}$

434.51

$172.99 \mathrm{~K}$

$1.34139 \AA$

Tetragonal

$\mathrm{P}_{3}$

$\begin{array}{ll}\mathrm{a}=13.5313(3) \AA & \alpha=90^{\circ} . \\ \mathrm{b}=13.5313(3) \AA & \beta=90^{\circ} . \\ \mathrm{c}=12.2153(3) \AA & \gamma=90^{\circ} .\end{array}$

2236.57(11) $\AA^{3}$

4

$1.290 \mathrm{Mg} / \mathrm{m}^{3}$

$0.417 \mathrm{~mm}^{-1}$

920

$0.06 \times 0.06 \times 0.05 \mathrm{~mm}^{3}$

2.841 to $54.933^{\circ}$.

$-16<=\mathrm{h}<=16,-16<=\mathrm{k}<=13,-14<=\mathrm{l}<=14$

27061

$4238[\mathrm{R}(\mathrm{int})=0.0477]$

$99.3 \%$

Semi-empirical from equivalents

0.7508 and 0.6323

Full-matrix least-squares on $\mathrm{F}^{2}$

4238 / 2 / 303

1.062

$\mathrm{R} 1=0.0303, \mathrm{wR} 2=0.0726$ 
$\mathrm{R}$ indices (all data)

Absolute structure parameter

Extinction coefficient

Largest diff. peak and hole
$\mathrm{R} 1=0.0334, \mathrm{wR} 2=0.0744$

$0.02(10)$

$\mathrm{n} / \mathrm{a}$

0.113 and -0.129 e. $\AA^{-3}$ 